\title{
Influence of Flow and Spatial Heterogeneities on Pattern Formation of Dictyostelium discoideum
}

\section{Dissertation}

\author{
for the award of the degree \\ "Doctor rerum naturalium" \\ at the Georg-August-Universität Göttingen
}

within the doctoral degree programme

Physics of Biological and Complex Systems

of the Göttingen Graduate School of Neurosciences, Biophysics, and

Molecular Biosciences (GGNB)

of the Georg-August University School of Sciences (GAUSS)

submitted by

Estefania Vidal

from Santiago, Chile

Göttingen, 2019 


\section{Thesis advisory committee}

\section{Dr. Azam Gholami}

Laboratory of Fluid Physics, Pattern Formation, and Biocomplexity Max Planck Institute for Dynamics and Self-Organization

\section{Prof. Dr. Stefan Klumpp}

Institut für Dynamik komplexer Systeme

Georg-August-Universität Göttingen

\section{Prof. Dr. Jörg Enderlein}

Drittes Physikalisches Institut - Biophysik

Georg-August-Universität Göttingen

Members of the examination board:

\section{Referee:}

\section{Dr. Azam Gholami}

Laboratory of Fluid Physics, Pattern Formation, and Biocomplexity Max Planck Institute for Dynamics and Self-Organization

\section{Co-referee:}

Prof. Dr. Stefan Klumpp

Institut für Dynamik komplexer Systeme

Georg-August-Universität Göttingen

Other Members of the Examination Board:

\section{Prof. Dr. Jörg Enderlein}

Drittes Physikalisches Institut - Biophysik

Georg-August-Universität Göttingen

\section{Prof. Dr. Ulrich Parlitz}

Biomedical Physics Group

Max Planck Institute for Dynamics and Self-Organization

\section{Prof. Dr. Annette Zippelius}

Institut für Theoretische Physik

Georg-August-Universität Göttingen

\section{Dr. Karen Alim}

Biological Physics and Morphogenesis Group

Max Planck Institute for Dynamics and Self-Organization 
Mister Sondheim! Look, I made a hat! Where there never was a hat!

Lin-Manuel Miranda 



\section{Contents}

$\begin{array}{lr}\text { Abstract } & 1\end{array}$

1 Introduction $\quad 3$

1.1 Dictyostelium discoideum . . . . . . . . . . . . . . . . . . . . . 3

1.2 Reaction - Diffusion Systems _. . . . . . . . . . . . . . . . . 8

1.2.1 Oscillatory Systems . . . . . . . . . . . . . . . . . 8

1.2.2 Excitable Systems . . . . . . . . . . . . . . . . . . . . 12

1.3 The Martiel-Goldbeter Model . . . . . . . . . . . . . . . . . . . . . . . . . 15

1.3.1 3-Component Martiel-Goldbeter Model . . . . . . . . . . . . . . . . . 15

1.3.2 Breaking Translational Invariance . . . . . . . . . . . . . . . . . . . . 19

1.3.3 2-Component Martiel-Goldbeter Model . . . . . . . . . . . . . . . . 22

1.3.4 Modifications to the Model in the Presence of Caffeine . . . . . . . . . . . . . . 23

1.4 Other D. discoideum Model . . . . . . . . . . . . . . . . . . . . . . . . 23

1.4.1 Kessler-Levine Model . . . . . . . . . . . . . . . . . . . . . . . . . 24

1.5 Motility Models . . . . . . . . . . . . . . . . . . . . . . . . . . 26

1.6 Computational Methods . . . . . . . . . . . . . . . . . . . . . . . 28

1.6.1 Finite Differences . . . . . . . . . . . . . . . . . . . . 28

1.6.2 Runge-Kutta-Merson . . . . . . . . . . . . . . . . . . 31

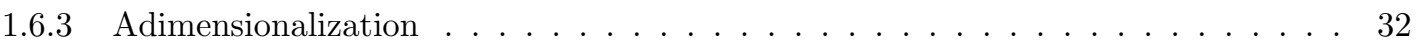

2 Convective Instability and Boundary Driven Oscillations 33

3 Influence of Fast Advective Flows on Pattern Formation in Dictyostelium discoideum

4 Spatial Heterogeneities Shape Collective Behavior of Signaling Amoeboid Cells 69

5 Spontaneous Center Formation in Dictyostelium discoideum 83

6 Discussion $\quad 95$

6.1 Perturbation by Advecting Flows . . . . . . . . . . . . . . . . . . . 95

6.2 Perturbation by Obstacles and Spontaneous Target Centers . . . . . . . . . . . . . . 100

7 Conclusions and Outlook $r$

Appendix: Comparison of Oscillatory Regime to Its Amplitude Equation 108 
List of Figures

Bibliography

119

Acknowlegments

129

Curriculum Vitae

131 


\section{Abstract}

In the present cumulative thesis the reaction-diffusion equations modeling the signaling process of the social amoeba Dictyostelium discoideum were studied. The pattern formation process in this organism, corresponding to the production and relay of waves of the chemoattractant cAMP, was perturbed under two particular conditions: advecting flows and millimetric obstacles. The model was studied through analytical calculations, when suitable approximations were possible, and numerical simulations. The results were compared to experimental observations in such setups.

In the first part of this work, the model was modified to account for an advecting flow being applied to the system, similar to those affecting the amoebas in their natural habitat. Under these conditions the system shows a convectively unstable regime which was fully characterized. In this regime a perturbation produces downstream traveling wave trains that grow in size as they travel. These wave trains have a smaller wavelength and lower velocity on their leading front than in the center, where the peaks are more spreaded out and travel faster. Adding an absorbing boundary condition on the upstream end of the channel creates an instability capable of periodically producing wave trains which are advected downstream. This periodic process also emits an upstream traveling peak which gets absorbed by the upstream boundary and whose velocity sets the oscillation period. In a two dimensional channel this upstream traveling peak acquires a triangular shape, with its cusp at the middle of the channel. This shape becomes more elongated with increasing advecting velocities and as the peak travels along the channel.

At high flow speeds the cAMP waves acquire a very elongated parabolic shape that the model with instantaneous cAMP transfer to the extracellular media could not reproduce, but that the full 3-Component model version with a developmental path scheme successfully reproduced. The shape of the wavefront was very dependent on the location of the wave initiation point, which could only happen in the upstream boundary at high advecting flows. In our simulations a big enough group of oscillatory cells needs to be located upstream to successfully produce waves, which is consistent with experimental observations. Both in experiments and simulations the wave width increased with increasing advecting velocities and reacted very quickly to speed changes in the 
advecting flow. The wave period was constant along the entire range of studied flow velocities, thus providing a robust feature for aggregation across different environmental conditions.

In the second part of this work a mechanism for the creation of target patterns in D. discoideum at densities below mono-layer was uncovered. By adding a discrete cell distribution to the reaction-diffusion equations, areas of higher cell density naturally become oscillators and produce traveling waves, while areas of lower density reach a low cAMP stable steady state that can be excited. This allows the waves emanating from the target centers to be relayed through the entire system. By adding cell movement the model showed ramifying aggregation streams, similar to those observed in experiments. If in these streams the local density goes above mono-layer (confluency) a local degradation mechanism is necessary to stop them from breaking apart. This degradation can exist in the form of membrane-bounded phosphodiesterase. This work shows that the apparition of target centers is a collective phenomenon and not the work of specialized groups of cells, therefore it is consistent with recent experimental observations.

By modifying parameters in this model, the effects of adding caffeine to the cells' buffer were successfully reproduced. Under these modifications the system showed longer oscillation periods, slower traveling waves, and fewer aggregation centers. Adding millimetric size pillars to this setup can impose specific locations for target pattern appearance, thus controlling the aggregation locations for the amoebas. For this technique to be successful the numerical simulations propose various mechanisms that might be in play acting as boundary conditions in the experimental setup. Simulations also showed an increase in sensitivity to cAMP with the addition of caffeine, thus making it easier for the amoebas to react to any possible cAMP accumulation around the pillars. These simulations provide new information on the sensitivity of D. discoideum to cAMP and open new venues for the control of multicellular aggregation. 


\section{Introduction}

\subsection{Dictyostelium discoideum}

Dictyostelium discoideum (D. discoideum) belongs to a group of organisms known as social amoebas. These amoebas feed on bacteria and live a solitary life as long as the nutrients (food supply) last. During nutrient depletion the cells begin an aggregation process in which they gather in groups of around $10^{4}$ to $10^{6}$ amoebas and form multicellular structures known as fruiting bodies in order to survive [1]. It is particularly remarkable that the growth and development phases in these organisms are strictly separated. During the growth phase, when the nutrients are plenty, the cells grow and reproduce through mitosis; while the necessary genes for aggregation are not expressed until the cells are starved [2].

Starvation triggers a series of changes in D. discoideum in which the genes used in the growth phase are down-regulated, while others are expressed. This includes the necessary genes to produce and react to the chemoattractant 3',5'-cyclic adenosine monophosphate (cAMP) [3]. This chemical is the signaling molecule used by D. discoideum to organize and aggregate large areas of up to $1 \mathrm{~cm}^{2}$. At roughly 3 hours into the starvation time cells start to emit pulses of cAMP which spread as traveling chemical waves through the system. The amoebas are capable of detecting the cAMP waves and react to it by both producing cAMP themselves, thus allowing the cAMP waves to propagate, and by moving against the wave propagation direction, towards the origin of the traveling wave.

This motion following a chemical cue is known as chemotaxis and works by measuring the concentration of cAMP along the whole scale of the amoeba. The cells detect the presence of cAMP using receptors located on the cell membrane and move in the direction of the higher concentration measured as the area of higher percentage of receptor occupancy (spatial sensing) [4]. Once it starts moving, the cell continues in the same direction for some time, showing chemotactical memory [5], therefore it ignores the decreasing concentration of cAMP once the peak of the wavefront passes. In other words, it does not reorient on the wave-back [6] and continues in the direction given by the wavefront. 

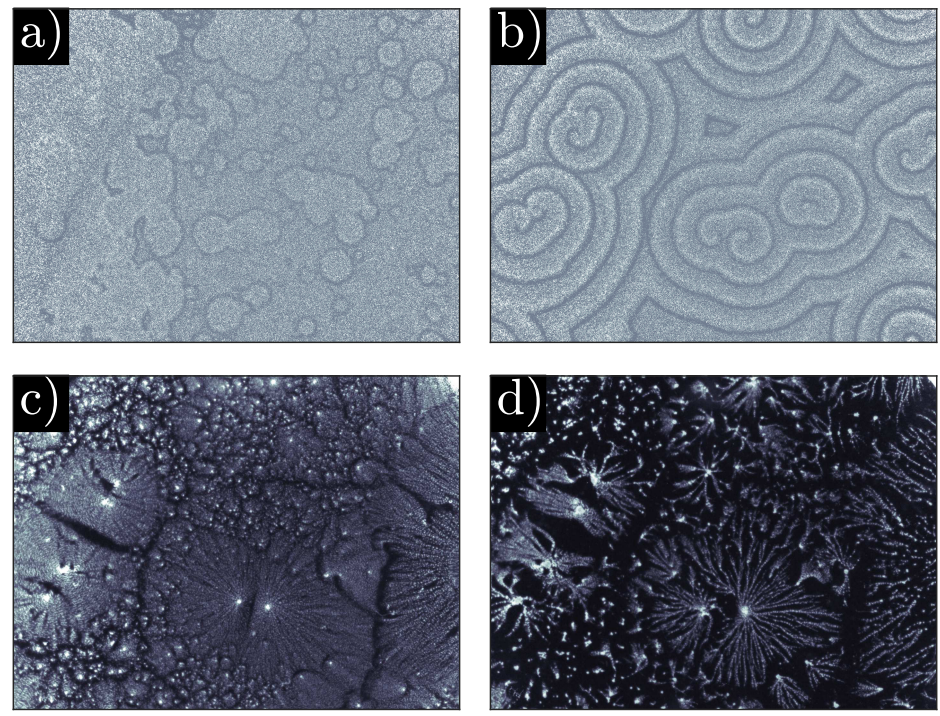

Figure 1.1: Processed dark-field microscopy images of a colony of cells during their development process. a) Initial circular pulses after $2.7 \mathrm{hrs}$ of starvation. b) Spiral patterns after $5 \mathrm{hrs}$ of starvation. c) Initial aggregation patterns, $10 \mathrm{hrs}$ of starvation. d) Clear aggregation streams, $13.9 \mathrm{hrs}$ of starvation. (Courtesy of Torsten Eckstein at Max Planck Institute for Dynamics and Self-Organization, unpublished).

Since the chemical waves are produced in periodical pulses, the amoebas' motion is discontinuous, occurring with each passing wave. In wild type amoebas the cAMP waves form rotating spiral waves and concentric target waves [7], these structures share the feature that they both have a center from which the waves emanate, thus giving a specific location towards which the cells move. Examples of these waves can be observed in Figure $1.1 \mathrm{a}-\mathrm{b})$.

During their journey towards the aggregation centers the amoebas form a very distinctive pattern known as aggregation streams. These streams consist in the tail-to-head alignment of cells, forming lines of cells that branch out from the aggregation centers. These structures can be seen in Figure $1.1 \mathrm{c}-\mathrm{d}$ ). Up to this stage the cells maintain their individuality and the process is reversible at anytime by feeding nutrients to the amoebas. Around 4 to 6 hours into starvation the cells lose their phagocytic function and the capacity to go back to the growth phase, therefore they continue to develop even if they are supplied with nutrients [8].

Once the cells aggregate in a location, they form a multicellular structure resembling a slug, where a group of cells differentiate to form the tip of the structure. This tip becomes the source of the signals used for organizing and therefore controls development [9]. At this stage the multicellular structure receives the name of slug, and can migrate as a single organism. The slug is phototactic and moves in shallow heat gradients [10].

The final step in the survival process is known as culmination and it occurs when the slug stands up and forms a fruiting body, with the front of the slug forming the stalk (20\% of the slug's cells), and the rear, the spores (80\%) [9]. The spores are dormant, 
but the stalk's cells are dead, thus sacrificing $20 \%$ of the colony to ensure its survival. The spores can then be released to the environment, producing the next generation of amoebas. A schematic representation of these phases of D. discoideum's life cycle is presented in Figure 1.2.

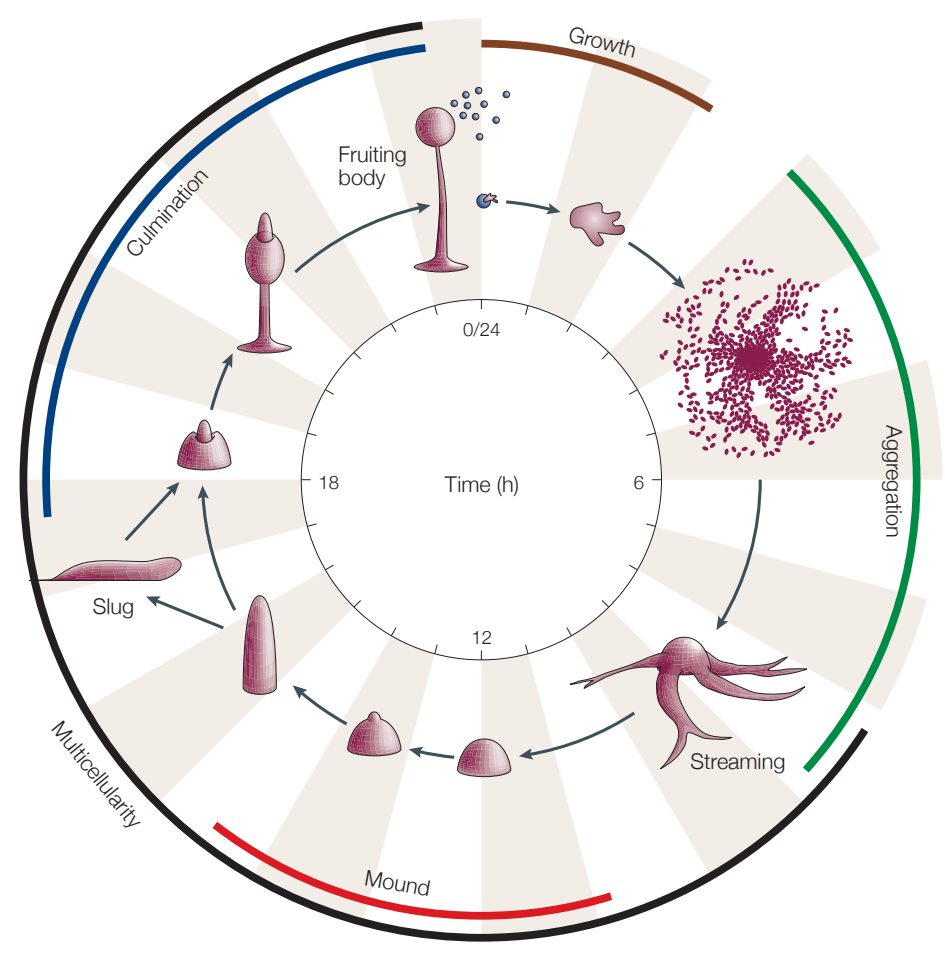

Figure 1.2: Life cycle of $D$. discoideum showing the development that occurs under starvation conditions: pattern formation, aggregation, mound, slug, and fruiting body. Reproduced with permission of the publisher from the work of Chisholm and Firtel [11].

The first observations of Dictyostelium amoebas are due to Brefeld [12], who in 1869 reported the observation of aggregating territories and fruiting bodies in D. mucoroides. D. discoideum itself was not discovered until 1935 by Raper [13] who not only isolated D. discoideum but also discovered that they would feed on almost any bacteria, which made the manipulation of $D$. discoideum much easier than the other previously observed Dictyostelium species, thus increasing the studies centered in the organism. Raper also found that the percentage of cells who would produce the stalk (20\%) was independent of the size of the slug, and that different species of Dictyostelium do not mix and therefore aggregate separately [14].

The existence of a chemoattractant molecule to regulate aggregation was proposed in 1947 [15] and was identified to be cAMP in 1968 [16]. The mechanism for cAMP wave propagation was proposed by Shaffer [17], who also identified the molecule responsible for cAMP degradation, phosphodiesterase, and the fundamental role performed by this degradation. Without phosphodiesterase the amount of cAMP would accumulate in the extracellular media, making it impossible to achieve the gradients necessary for 
aggregation without continuously increasing the cAMP production. He proposed that cells react to detecting cAMP by synthesizing and releasing cAMP to the extracellular media, thus allowing, in conjunction with cAMP degradation, to produce and relay waves with strong gradients that can travel through the system. The relay method proposed by Shaffer is an energetically efficient process, since the waves get degraded and produced as they spread, and does not require as much cAMP as if it were only one big cAMP release at the aggregation center.

This thesis focuses on modeling the signaling phase of D. discoideum aggregation, therefore the slug and fruiting body stages will not be further discussed. For an in-depth review of these stages and other aspects of Dictyostelium genetics, refer to Richard H. Kessin's review [1] and references therein.

To close this brief review of $D$. discoideum the reactions underlying cAMP production will be outlined. D. discoideum detects the presence of extracellular cAMP through cAMP receptors located in the exterior of the cellular membrane. There are four proteins on the cell membrane for which their extracellular ligand is cAMP, named cAR1, cAR2, cAR3, and cAR4 (cAR stands for cyclic AMP receptor). Knockout experiments have shown that the most relevant of these proteins for early aggregation is cAR1 [18]. Lack of cAR1 blocks the ability to sense cAMP, bind to it, and consequently further development [19]. It has also been shown that cells lacking cAR1 can not be rescued through the earlier expression of other receptors such as cAR2 or cAR3 [20].

The other cAMP receptors take a more fundamental role at later stages of development. It has been shown through knockout mutants that cAR4 [21] and cAR2 [22] do not play an important role during aggregation, with cAR2 being specially expressed in prestalk cells [22], and cAR4 more relevant in the transition from mound to slug [21]. cAR3 concentration peaks during late aggregation, around 12 hours into starvation [20]. Therefore all these receptors are necessary for the correct development of $D$. discoideum, but cAR1 is the most relevant for early aggregation.

Once the cAMP receptors bind to cAMP, the amoeba experiences a reduction in the number of binding sites [23]. This has been explained as the receptors going through a phosphorylation process [24]. In this phosphorylated state the affinity for cAMP is reduced 5-fold [1], in a process known as desensitization. This desensitization is reversible once the receptors are no longer exposed to cAMP [25], that is, the receptors can revert to their dephosphorylated (high affinity) state. This reverse process is known as resensitization or deadaptation. The rates at which these processes occur in the presence of cAMP have been estimated at $0.222 \mathrm{~min}^{-1}$ for desensitization and 0.055 $\min ^{-1}$ for resensitization [25]. The speeds of these two timescales compared to the cAMP production and release timescale (0.34-0.94 $\mathrm{min}^{-1}$ [26]) provide the excitable characteristics to this system, as will be later shown in Section 1.3. 
The other important process triggered by binding cAMP is the start of the chain of events that leads to the production and release of cAMP. This complex signal transduction pathway also controls chemotaxis towards the source of cAMP, among others. The details of this pathway are extensively presented in a recent review by Devreotes et al. $[27]$.

The intracellular cAMP is produced as a reaction of ATP catalyzed by the enzyme Adenylyl Cyclase (also named Adenylate Cyclase or ACA). This enzyme has a hundred times larger affinity for the substrate ATP when it is in its activated form, than in its free-form [28]. This activation of the ACA occurs down the signal pathway started by the binding of cAMP to cAR1. cAR1 interacts with $\mathrm{G} 2$ which is an heterotrimeric G-protein, which then activates $\mathrm{AC}$ through the cytosolic regulator of adenylyl cyclase (CRAC) [29]. In D. discoideum three types of adenylyl cyclases have been identified: ACA, ACB, and ACG. Of these three only ACA produces cAMP during early aggregation, while $\mathrm{ACG}$ is an osmosensor that controls the germination of spores [30], and ACB activity peaks at the beginning of the formation of the fruiting body [31].

Once cAMP has been produced in the inside of the cell, it is subject to degradation by intracellular phosphodiesterase which decomposes it into adenosine monophosphate (AMP). D. discoideum has seven types of phosphodiesterases, two of which (PDE2 and PDE6) act in the intracellular media, degrading intracellular cAMP [32]. The final step in the relaying process is the release of cAMP to the extracellular media. The release rate is proportional to the intracellular cAMP levels, consistent with a first order reaction [26]. This secreted cAMP can then activate other cells, allowing signal propagation.

One modification to the signaling process of particular relevance in Chapter 4 of this thesis [33] is the addition of caffeine to the buffer in which cells are starved. It has been shown that caffeine inhibits the synthesis of cAMP [34], both the autonomous production (pacemakers) and the reaction to a cAMP pulse. This inhibition maintains cell viability, that is, the amoebas are healthy and still capable of aggregation, even more, this process is reversible, with the cells returning to their normal production rate after caffeine has been removed [35]. Under the effects of caffeine the oscillating period increases from 5-6 mins to 9-10 mins [36]. It has also been observed that the radius of the spiral core increases with increasing caffeine concentrations, therefore the amoebas form a ring of higher cell density, instead of the mound they would form without caffeine [36]. Of particular importance is that the cells' sensitivity to cAMP increases in the presence of caffeine [34], that means, that the cells are capable to react to smaller cAMP concentrations than without caffeine. This has been explained as a consequence of the overall decrease of cAMP in the system [34]. Since the cells react to cAMP increases in amounts comparable to the base value of cAMP, they detect smaller increases when the base value is lowered. The exact way in which caffeine affects $D$. discoideum has not 
been found [37]. However, it has been shown that caffeine does not activate any kind of phosphodiesterase in D. discoideum [37], which could explain the decrease in cAMP. In enzyme essays it has been shown that caffeine inhibits the activation of ACA [37], while in the intact cells it inhibited all three kinds of ACs (ACA, ACB, and ACG). This suggested more than one target for caffeine, one that inhibits the activation of ACA by GTP, and one that acts globally affecting all ACs [37]. Therefore, further research needs to be conducted to fully understand the effects of caffeine in D. discoideum.

\subsection{Reaction - Diffusion Systems}

Before diving into the particularities of the model used to describe the cAMP waves in D. discoideum it is necessary to put such model into the broader context of reactiondiffusion systems. These systems, as their name suggests, were initially used to describe chemical reactions in which the reactants also have the capability of diffusing, but their scope is nowadays much larger. From a mathematical point of view we will use this term to refer to coupled partial differential equations which are first order in time, contain at least one diffusive field, and usually present some non-linearity. Of particular importance for this work are reaction-diffusion systems that show sustained oscillations and excitable systems.

\subsubsection{Oscillatory Systems}

The first oscillatory chemical reaction was discovered in 1951 by Boris Belousov [38] (reference in Russian, a similar manuscript of 1951 was translated to English [39]), but it did not attract much attention at the time, since referees and other scientists thought that it violated the second law of thermodynamics [40, 41]. In his work, Belousov described a chemical reaction which changed color periodically between yellow and transparent, due to the oscillating presence of $\mathrm{Ce}^{+4}$ and $\mathrm{Ce}^{+3}$, respectively. Anatol Zhabotinsky would later obtain a better formulation of the reaction, in which no precipitate was created $[40,42]$, which allowed him to observe traveling waves in the unstirred system. This reaction is what we call the Belousov-Zhabotinsky (BZ) reaction, which has been since then largely studied for the interesting structures it shows, some of which can be observed in Figure 1.3.

The reconciliation of oscillatory systems with thermodynamics is due to Ilya Prigogine [41] who presented in 1968 chemical schemes in which deviations from the steady state had a negative "excess entropy production" due to autocatalytic or cross-catalytic reactions [44], thus departing from the homogeneous steady state in a way compatible with thermodynamics. That is, moving away from the equilibrium position would diminish the entropy production, allowing the existence of stable solutions with time dependent concentrations. The first of the examples presented in his work was the 

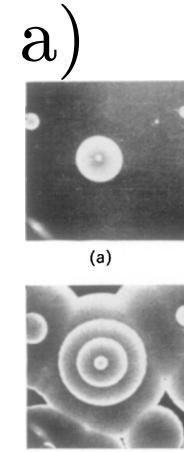

(e)

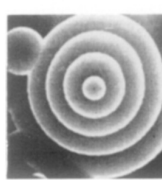

(i)

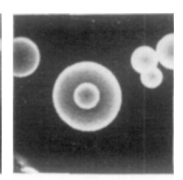

(b)

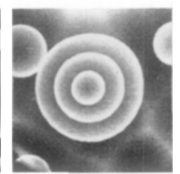

(f)

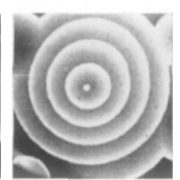

(j)

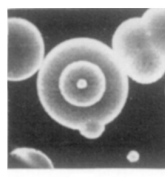

(c)

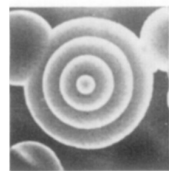

(g)

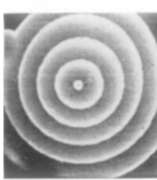

(k)

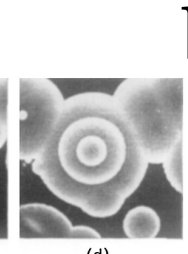

(d)

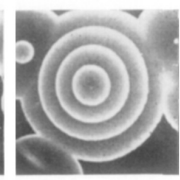

(h)

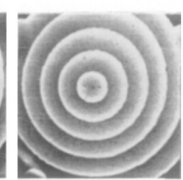

(I) b)
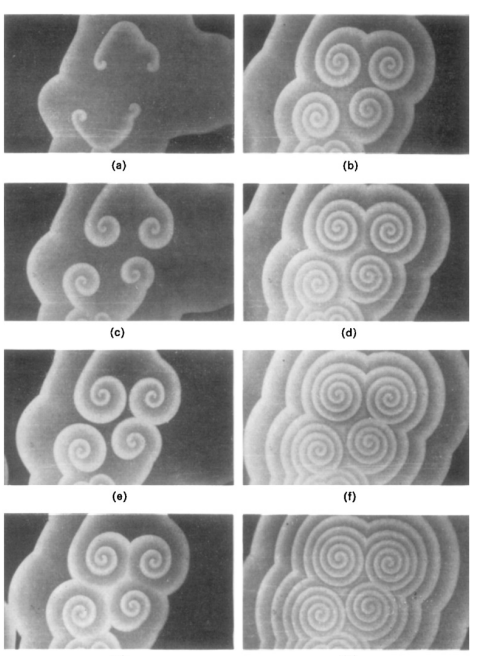

Figure 1.3: Experimental observations of the Belousov-Zhabotinsky reaction, reproduced with permission of the publisher from the work of Zhabotinsky and Zaikin [43]. a) Target patterns. b) Spiral waves.

reaction

$$
\begin{aligned}
A & \stackrel{k_{1}}{\longrightarrow} X, \\
2 X+Y & \stackrel{k_{2}}{\longrightarrow} 3 X, \\
B+X & \stackrel{k_{3}}{\longrightarrow} Y+D, \\
X & \stackrel{k_{4}}{\longrightarrow} E,
\end{aligned}
$$

where the reverse reactions were neglected. This system can be modeled by the pair of equations describing the fields $X$ and $Y$

$$
\begin{aligned}
\partial_{t} X & =k_{1} A+k_{2} X^{2} Y-k_{3} B X-k_{4} X+D_{X} \partial_{r r} X, \\
\partial_{t} Y & =k_{3} B X-k_{2} X^{2} Y+D_{Y} \partial_{r r} Y,
\end{aligned}
$$

where concentrations of the initial reactants $A, B$ and of the final products $D, E$ are assumed constant. This system shows oscillatory behaviour and traveling waves, as can be seen in Figure 1.4. Due to its simplicity, this pair of equations was widely used to study this type of structures and became known as the Brusselator. Note that an autocatalytic or cross-catalytic reaction is necessary to have a nonlinear term in the differential equations, which is fundamental for the appearance of these structures. Interestingly, due to its high complexity, the kinetic equations taking place in the BZ reaction were not described until 1972 [45].

We use now the term oscillatory system to describe systems of equations that present a stable limit cycle, usually appearing through a Hopf bifurcation. In this bifurcation a stable steady state becomes unstable when a pair of eigenvalues cross the imaginary 

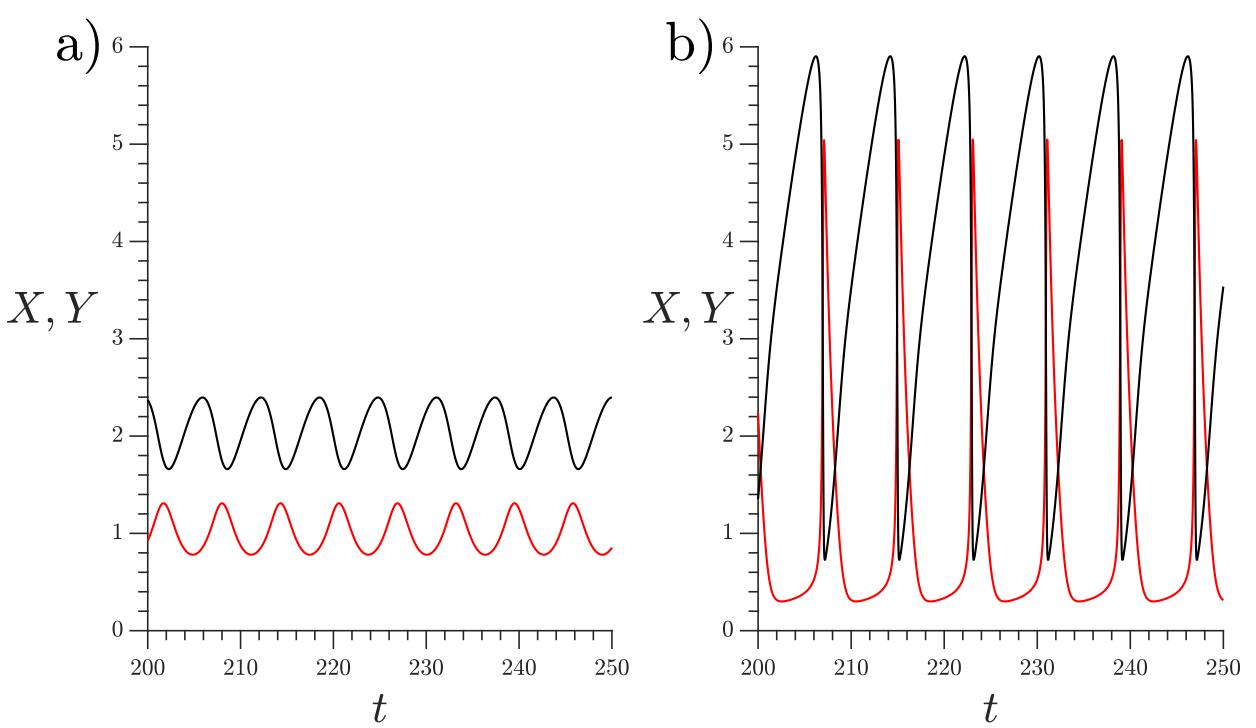

Figure 1.4: Numerical simulations of the Brusselator, Equation 1.1, showing oscillatory behavior at different parameters. All kinetic constants are taken as equal, $A=1$, and diffusion is neglected. a) $B=2.05$, b) $B=3.5$. Concentration of $X$ in black, concentration of $Y$ in red.

axis with a non-zero imaginary part. The limit cycle exists around the unstable steady state and its characteristics like shape and frequency depend on the system parameters and not on initial conditions (as opposition to, for example, the Lotka-Volterra model in which the shape of the limit cycle depends on initial conditions [46]). Two examples of limit cycles in the Brusselator model are shown in Figure 1.4.

After these pioneering works much has been done in the field of oscillatory systems, here we only summarize some of those that are relevant to the structures observed in D. discoideum.

The differently colored bands initially observed in the oscillatory BZ reaction were shown to be traveling waves [47] which existed due to a gradient in temperature or in reactant concentration, and were, therefore, independent of diffusion. The theory of traveling waves in oscillatory systems was described in 1973 by Kopell and Howard [48] for the $\lambda-\omega$ system. These waves are known as phase waves and exist due to phase gradients between coupled oscillators, they are largely independent of diffusion, and have a variable speed, which is not intrinsic to the system and can be arbitrarily large [47]. An example of these waves due to concentration gradients is shown in Figure 1.5 a).

In a symmetric 2-D system these waves form target patterns, which are concentric expanding circular waves. To break the symmetry of the system, these target patterns need an oscillating core with a different frequency than the rest of the system [49]. If the oscillating core has a higher frequency it produces outgoing waves, while a lower frequency one would produce inward traveling waves [50]. The effect of a core oscillating 
at a higher frequency than the bulk is shown in Figure $1.5 \mathrm{~b}$ ) where the waves emanating from the center can be observed. Both in the BZ reaction and in D. discoideum target patterns appear in a range of different frequencies [51]. A target pattern with higher frequency would expand its entrainment area, i.e., the waves would reach farther away with each new wave, taking over other lower frequency centers [52].
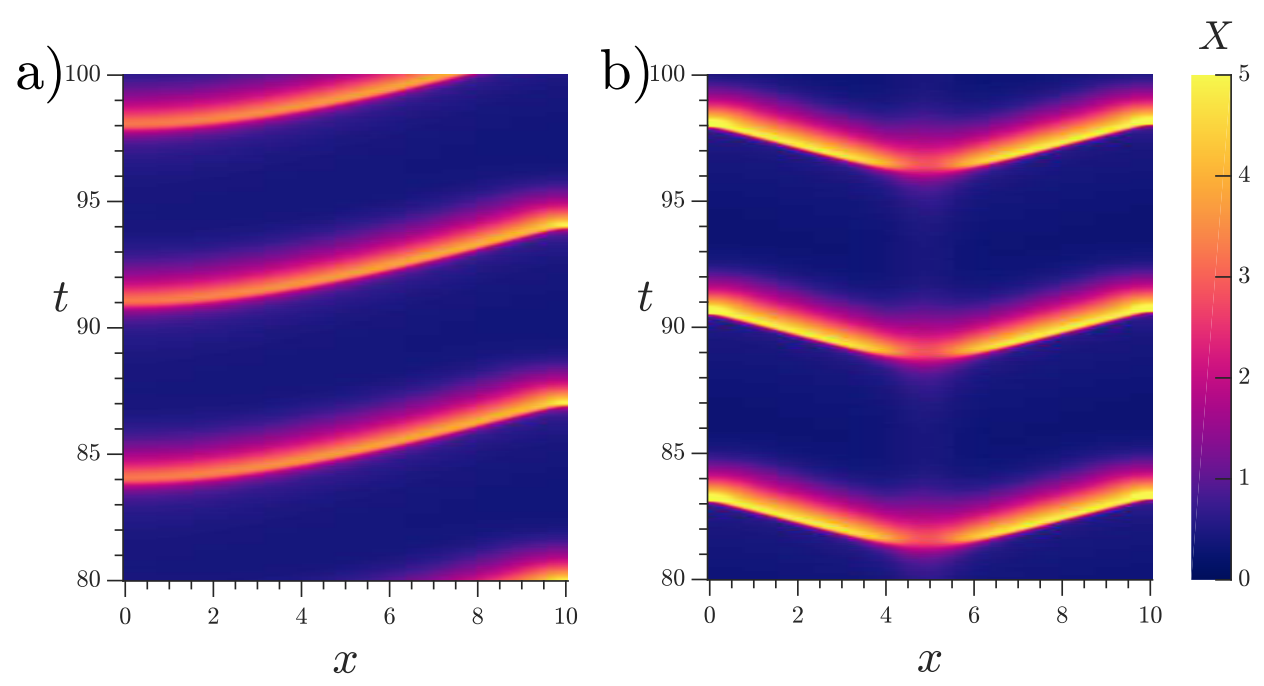

Figure 1.5: Typical features of oscillatory systems in the Brusselator model. a) Phase waves due to gradients in reactants' concentration. Concentration of $B$ increases linearly from 2.8 at $x=0$ to 3.2 at $x=10$. b) Center emitting target waves due to a higher frequency limit cycle. $B=2.5$ in $4.5<x<5.5, B=3.5$ everywhere else. $D_{X}=0.2, D_{Y}=0.02$. All other parameters as in Figure 1.4. Colormap by Peter Kovesi [53].

Another structure that has been observed in D.discoideum, the BZ reaction, and other oscillatory systems is the spiral wave. This type of wave has a free end which curves around itself and rotates around a center with a fixed period. It was theoretically described for the $\lambda-\omega$ system by Cohen in 1978 [54], who showed the existence of logarithmic spirals, and by Greenberg [55], who showed the existence of Archimedian spirals. These dissipative structures are characterized by having a topological charge, which can be described as the amount of arms the spiral has, while the sign of the charge gives the spiral's direction of rotation. Mathematically, the charge is defined by the path integral around the phase discontinuity such that

$$
m=\frac{1}{2 \pi} \oint_{\Gamma} \nabla \varphi
$$

where $m$ is the spiral's topological charge, $\varphi$ its phase, and $\Gamma$ is a closed path around the singularity. A representative image of these types of spirals with $m=1$ is shown in Figure 1.6. In signaling colonies of $D$. discoideum only spirals with topological charge $m= \pm 1$ have been observed, that means that only one-armed spirals appear naturally. Nevertheless, double-armed spirals have been stabilized during starvation with the help of millimetric obstacles [33]. In later stages of development, where the cells have already 

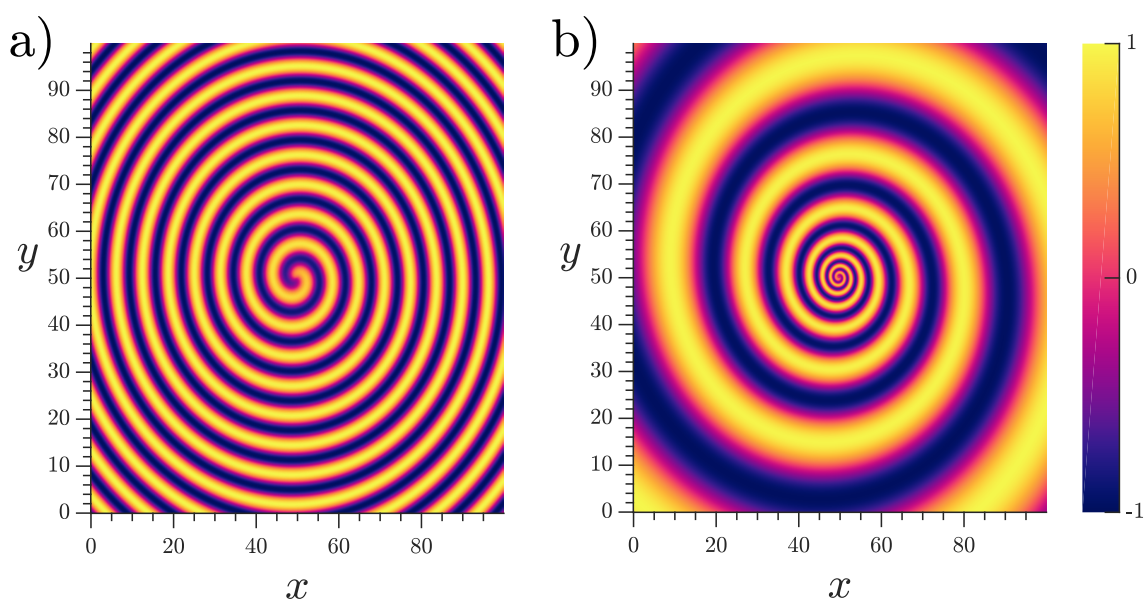

Figure 1.6: Two types of spirals that can be observed in oscillatory systems. a) Archimedian spiral. b) Logarithmic spiral.

formed a mound, double-armed spirals of cAMP waves have been reported [56].

As a final note on oscillatory systems it is worth mentioning the Complex GinzburgLandau (CGL) Equation. This equation describes the evolution of any system close to a Hopf-bifurcation. Sufficiently close to the bifurcation the separation of scales allows to adiabatically eliminate the fast variables, and the system can be described by a couple of slow variables, thus giving this equation a universal character. This equation was first proposed by Landau in 1944 [57] in the context of turbulence description and it can be rigorously derived from reaction-diffusion equations [58]. The CGL Equation for the amplitude $A$ of oscillations is

$$
\partial_{t} A=A-(1+i \alpha) A|A|^{2}+(1+i \beta) \nabla^{2} A
$$

where $A$ is a complex field, $\alpha$ and $\beta$ are real parameters, and $\nabla^{2}$ is the Laplacian of the system.

Due to the universality of this equation it has been extensively studied to describe oscillatory systems. It has been shown that, depending on the parameter range, it can have traveling waves, spiral waves, and defect turbulence as solutions, among others. For a comprehensive review of this equation refer to the work of Aranson [59].

\subsubsection{Excitable Systems}

An excitable system has a steady state solution that is stable to small perturbations, but highly responsive to perturbations bigger than a certain threshold. If a supra-threshold perturbation is applied the system returns eventually to its steady state, since it is, after all, a stable fixed point, but instead of dampening out the perturbation quickly, it produces first a big response (see Figure 1.7). After this response, the system is usually refractory, that is, it can not be excited again until it recovers. Among the systems that 
present these characteristics are the non oscillatory Belousov-Zhabotinsky reaction [60], electrical activity in neurons [61], and the contraction waves in the cardiac muscle [62].
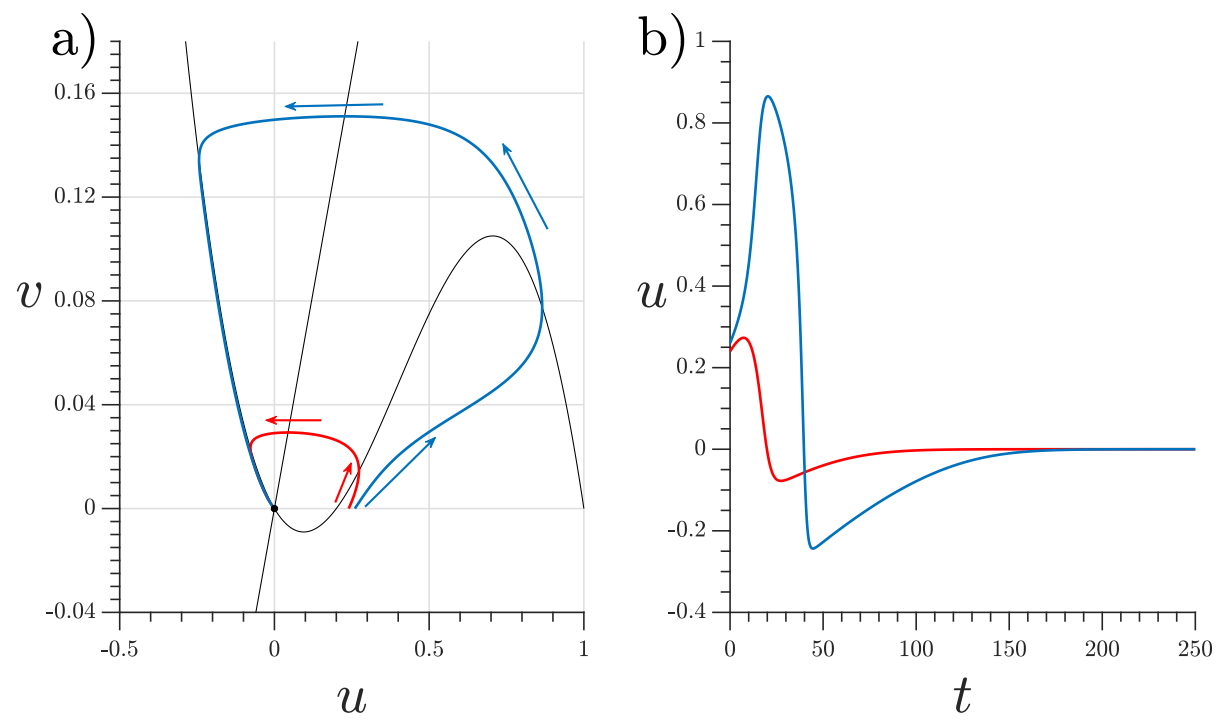

Figure 1.7: Excitable behavior in the FitzHugh-Nagumo model, Equation 1.3. Red simulation shows the reaction to an infra-threshold perturbation, blue simulation to a supra-threshold perturbation. a) Phase portrait showing both simulations and the nullclines $v=u / \beta$ and $v=u(u-\alpha)(1-u)$ in black. An infra-threshold perturbation (red line) decays quickly to the steady state $u_{0}=0, v_{0}=0$, a suprathreshold perturbation produces a big reaction from the system (blue line). b) Concentration of $u$ over time. Parameters are $\alpha=0.2, \beta=1.5, \epsilon=0.008$, initial conditions are for the red curve $u=0.24, v=0$ and for the blue curve $u=0.26, v=0$.

In extended excitable systems when a perturbation bigger than the threshold is applied the big reaction at the perturbation location diffuses through the system exciting the system in other locations. Therefore, in a one dimensional system a perturbation produces two pulses traveling in opposite directions, away from the perturbation. In two dimensions, a perturbation produces an expanding circular wave traveling with a curvature dependent speed.

More complex structures can be created by, for example, periodically perturbing the system in the same location. This creates a train wave in 1-D or a target pattern in 2-D. The frequency of the wave train is given by the perturbation frequency as long as the perturbation is slow enough so that the system can react. That is, the maximum frequency response of the system is given by the refractory period. The dispersion relation $c=f(T)$ where $c$ is wave velocity and $T$ wave period of such a wave train has a characteristic shape associated with trigger waves [64] that can be seen in Figure 1.8 a). This curve $f$ is for most excitable systems monotonically increasing, if not, the system is referred as to having an anomalous dispersion relation. For big periods $f$ converges to the single pulse velocity, since the more space pulses have between them, the less they affect each other, and therefore at high periods each peak of the wave train behaves mostly like a solitary pulse. $f$ has a minimum $T$ for which it is defined, this period 
a)

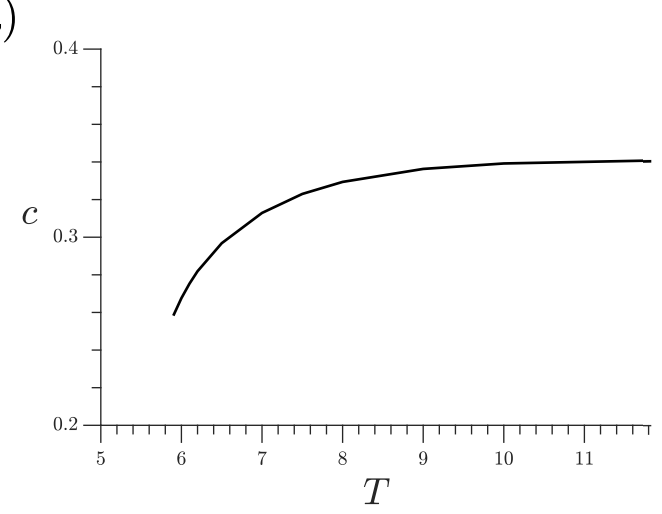

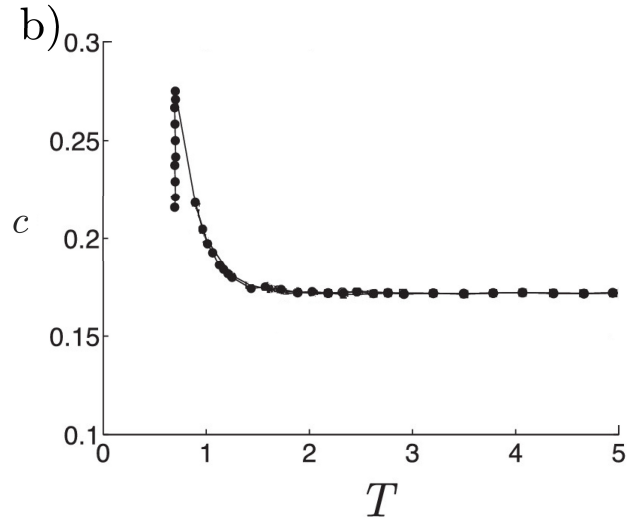

Figure 1.8: a) Example of a typical dispersion relation $c=f(T)$ for a planar trigger wave in an excitable system. $c$ is wave velocity and $T$ is the wave period. b) Example of an anomalous dispersion relation in the Kessler-Levine model. Part b) reproduced with minor modifications from the work of Oikawa et al. with permission of the publisher [63]

corresponds to the minimum period (maximum frequency) the system can relay.

Under controlled initial conditions a spiral wave can be created in this system. In a spiral wave the open end of a wave front produces a spiral tip, which curves to propagate into areas which are in the excitable state, in contrast to the areas just passed by the wave which are in a refractory state. The core of the spiral corresponds to the circular motion done by the spiral tip and the radius of this core depends on the length of the refractory period of the system. Unlike target patterns, spiral waves appear only in one particular frequency, which depends on the system's parameters. The problem of how this frequency is selected in spiral waves is complicated, and has been addressed by various authors [65-68]. In the particular case of experiments with wild type D. discoideum the selected spiral frequency is higher than the one of target patterns, therefore when both types of structures are present spirals dominate the system's dynamic by taking over target centers.

Unlike systems close to a bifurcation where a systematic separation of scales exists and an amplitude equation can be derived, in excitable systems such a model equation does not exist. Throughout the years different models have been used to derive and calculate more general properties of excitable systems, among those models particularly popular are the FitzHugh-Nagumo model and the Berkley model.

The FitzHugh-Nagumo model was proposed by Richard FitzHugh in 1961 [69] as a representative model of excitable-oscillatory systems. Indeed, this model can show periodic oscillations or excitable behavior depending on the choice of parameters. The second part of the name comes from the work of Nagumo et al. [70] who constructed an electrical circuit that can be described by this model. The dynamical equations of the two fields $u(x, y)$ and $v(x, y)$ are 


$$
\begin{aligned}
\partial_{t} v & =\epsilon(u-\beta v)+D_{v} \nabla^{2} v \\
\partial_{t} u & =u(u-\alpha)(1-u)-v+D_{u} \nabla^{2} u
\end{aligned}
$$

where $\alpha$ and $\beta$ are system parameters, $D_{u}$ and $D_{v}$ are the diffusion coefficients of $u$ and $v$, respectively; and $\epsilon \ll 1$ gives the timescale to the slow variable $v$, while $u$ is the fast variable.

In a similar fashion the Barkley model [71] describes two fields, $u$ which is the fast field also known as the excitation variable, and $v$ which is the slow field or recovery variable

$$
\begin{aligned}
\partial_{t} u & =\epsilon^{-1} u(1-u)[u-(v+\beta) / \alpha]+D_{u} \nabla^{2} u, \\
\partial_{t} v & =u-v,
\end{aligned}
$$

where again $\alpha$ and $\beta$ are system parameters and $\epsilon$ is chosen to be small. Note that only $u$ diffuses and $v$ does not. This has to do with the original intention of the author which was to capture the behaviour of neurons and cardiac tissue, where the recovery variable (in those cases, the tissue itself) does not diffuse. They also advice caution when using these equations to model chemical reactions where the diffusion rates of the components are of comparable sizes [71].

Extensive literature exists about the mathematical description of the structures present in excitable systems, in particular in the limits where the slow variable is not diffusive $\left(D_{v}=0\right)$ or when the diffusion rates are comparable $\left(D_{u} / D_{v} \approx 1\right)$. We refer the reader for further details to the works of Zykov [72], Tyson [65], Keener [73], and Fife [74].

\subsection{The Martiel-Goldbeter Model}

\subsubsection{3-Component Martiel-Goldbeter Model}

The model used in this work to describe the behavior of cAMP waves is usually referred to as the Martiel-Goldbeter model, since it was proposed by Jean-Louis Martiel and Albert Goldbeter in 1987 [75]. In their seminal work Martiel and Goldbeter reduced an original system of 10 kinetic equations to only 3 partial differential equations which govern the system. They also showed under which conditions the system can be further reduced to only two differential equations.

Summarizing the cAMP production and relay mechanism described in Section 1.1 the processes undergoing for the production of cAMP are, according to Martiel and Goldbeter [75], as follows. cAMP receptors are located on the outside of the cell membrane. They can exist in two forms, a dephosphorylated and a phosphorylated one and can change reversibly between these two states. These receptors bind with the cAMP present in the outside media with different affinities (at different rates). The de- 
phosphorylated form is more likely to bind with cAMP than the phosphorylated form, because of this, we will refer to them as the active and inactive forms, respectively. The active complex of binded cAMP with the active receptor activates the enzyme Adenylyl Cyclase (ACA), this process is particularly chosen to be nonlinear, requiring two receptors to activate one molecule of adenylyl cyclase. Note that the inactive receptors can not activate the enzyme. Then cAMP is synthesized from ATP, catalyzed by ACA, with the activated form of ACA producing more cAMP than the inactive form. The produced cAMP is then transported to the outside media where it can diffuse and bind with the receptors of other amoebas, although it is worth mentioning that diffusion was not included in the original model by Martiel and Goldbeter, who analyzed the system without spatial dependency. Finally, the cAMP present both in the intra- and extracellular media can be hydrolyzed (degradated) by the enzyme phosphodiesterase.

The detailed reduction of the chemical species equations to the three variable system can be found in the appendix of the work by Martiel and Goldbeter [75]. The final three variables are $\rho$ the percentage of active receptors on the cell membrane, $\beta$ the amount of intracellular cAMP, and $\gamma$ the extracellular cAMP concentration. The equations that govern this system are

$$
\begin{aligned}
& \partial_{t} \gamma=k_{t} \beta / h-k_{e} \gamma, \\
& \partial_{t} \beta=q \sigma \alpha \Phi(\rho, \gamma) /(1+\alpha)-\left(k_{i}+k_{t}\right) \beta, \\
& \partial_{t} \rho=-f_{1}(\gamma) k_{1} \rho+f_{2}(\gamma) k_{1}(1-\rho),
\end{aligned}
$$

with

$$
\begin{aligned}
f_{1}(\gamma) & =\frac{1+\kappa \gamma}{1+\gamma}, \\
\Phi(\rho, \gamma) & =\frac{\lambda_{1}+Y^{2}}{\lambda_{2}+Y^{2}},
\end{aligned} \quad \text { and } \quad Y(\gamma, \rho)=\frac{\rho \gamma}{1+\gamma} .
$$

In Equation 1.4 a) $k_{t} / h$ describes how much of the intracellular cAMP concentration is transfered to the extracellular media, where $k_{t}$ stands for the transport rate of cAMP from the intra- to the extracellular media and $h$ is the ratio of the extracellular to the intracellular volume (higher $h$ values correspond to lower cell densities). The cAMP on the extracellular media is reduced by degradation through phosphodiesterase at a rate $k_{e}$.

In Equation 1.4 b) the production of intracellular cAMP is represented by the nonlinear function $\Phi, \alpha$ describes the explicit dependency of production on ATP concentration and is shown as a multiplying factor of $\Phi$. $\sigma$ stands for the maximum activity of ACA, and $q$ is the ratio between the Michaelis constant of the activated form of ACA and the dissociation constant of the cAMP-active receptor complex. These parameters account for the production of intracellular cAMP $\beta$, which is decreased by two processes, the 
degradation in the intracellular media which happens at a rate $k_{i}$ and the transport towards the extracellular media, which occurs at a rate $k_{t}$.

Finally, in Equation 1.4 c) $f_{1}$ represents the nonlinear process of desensitization and $f_{2}$ the inverse process of resensitization of the receptors, while the timescale of the process is given by $k_{1}$. This makes $\rho$ the slow variable of the system, which is fundamental for the properties displayed by it. A schematic image of these processes can be seen in Figure 1 of Chapter 2.

Through this work most parameters are kept fixed, while only two are varied to map the phase diagram of the system. These parameters are $k_{e}$, which is the rate of cAMP degradation in the extracellular media and $\sigma$ which is proportional to the production rate of cAMP in the intracellular media.

To calculate the phase diagram of the system first the steady states are calculated by numerically searching for the triplets $\left(\gamma_{0}, \rho_{0}, \beta_{0}\right)$ such that $\partial_{t} \gamma=\partial_{t} \beta=\partial_{t} \rho=0$. In the parameter plane we explored, the system presented one, two or three steady states depending on the chosen parameters. We then performed stability analysis for all the triplets found by calculating the eigenvalues of the system's Jacobian

$$
J_{i, j}=\left.\frac{\partial F_{i}}{\partial x_{j}}\right|_{x=\left(\gamma_{0}, \rho_{0}, \beta_{0}\right)^{T}}
$$

where $F_{1,2,3}$ are the right hand side of Equation $1.4 \mathrm{a}$ ), b), and c) respectively, $x=$ $(\gamma, \rho, \beta)^{T}$, and the derivatives are evaluated at the steady state values. The first derivatives are

$$
\begin{array}{llrl}
\frac{\partial F_{1}}{\partial \rho} & =0, & \frac{\partial F_{1}}{\partial \beta} & =k_{t} / h, \\
\frac{\partial F_{1}}{\partial \gamma} & =-k_{e}, & \frac{\partial F_{2}}{\partial \rho} & =\frac{2 \gamma_{0}^{2} \rho_{0} s\left(\lambda_{2}-\lambda_{1}\right)}{\left(\gamma_{0}+1\right)^{2}\left(\lambda_{2}+Y^{2}\right)^{2}} \\
\frac{\partial F_{2}}{\partial \beta} & =-\left(k_{i}+k_{t}\right), & \frac{\partial F_{2}}{\partial \gamma} & =\frac{2 s \rho_{0}^{2} \gamma_{0}\left(\lambda_{2}-\lambda_{1}\right)}{\left(1+\gamma_{0}\right)^{3}\left(\lambda_{2}+Y^{2}\right)^{2}} \\
\frac{\partial F_{3}}{\partial \rho} & =-\frac{k_{1}\left(\mathcal{L}_{1}+\mathcal{L}_{2} c \gamma_{0} \kappa\right)}{c \gamma_{0}+1}-\frac{k_{1}\left(\gamma_{0} \kappa+1\right)}{\gamma_{0}+1}, & \frac{\partial F_{3}}{\partial \beta} & =0, \\
\frac{\partial F_{3}}{\partial \gamma} & =\frac{-k_{1} \rho_{0}(\kappa-1)}{\left(\gamma_{0}+1\right)^{2}}+\frac{c k_{1}\left(\rho_{0}-1\right)\left(\mathcal{L}_{1}-\mathcal{L}_{2} \kappa\right)}{\left(c \gamma_{0}+1\right)^{2}} & & \text { and }
\end{array}
$$

If the three eigenvalues of a steady state have negative real part that steady state is stable, while if at least one eigenvalue has a positive real part, that steady state is unstable. Based on the amount of steady states and the eigenvalues of the Jacobian four different regimes can be differentiated:

- Stable regime: The system has one steady state, which is stable. A perturbation 
applied to the system decays exponentially when time dependent simulations are performed.

- Bistable regime: The system has two steady states, both are stable.

- Oscillatory regime: The system has only one steady state, which is unstable. Time dependent simulations show a stable limit cycle around the unstable state.

- Excitable regime: The system has 3 steady states. Two unstable and one stable. Time dependent simulations show that the stable state is excitable, meaning that if a supra-threshold perturbation is applied the system does a big excursion before coming back to the steady state.

The parameter ranges of these regimes are shown in Figure 1.9 for the $k_{e}-\sigma$ plane. This work focuses particularly in the oscillatory and excitable regimes.
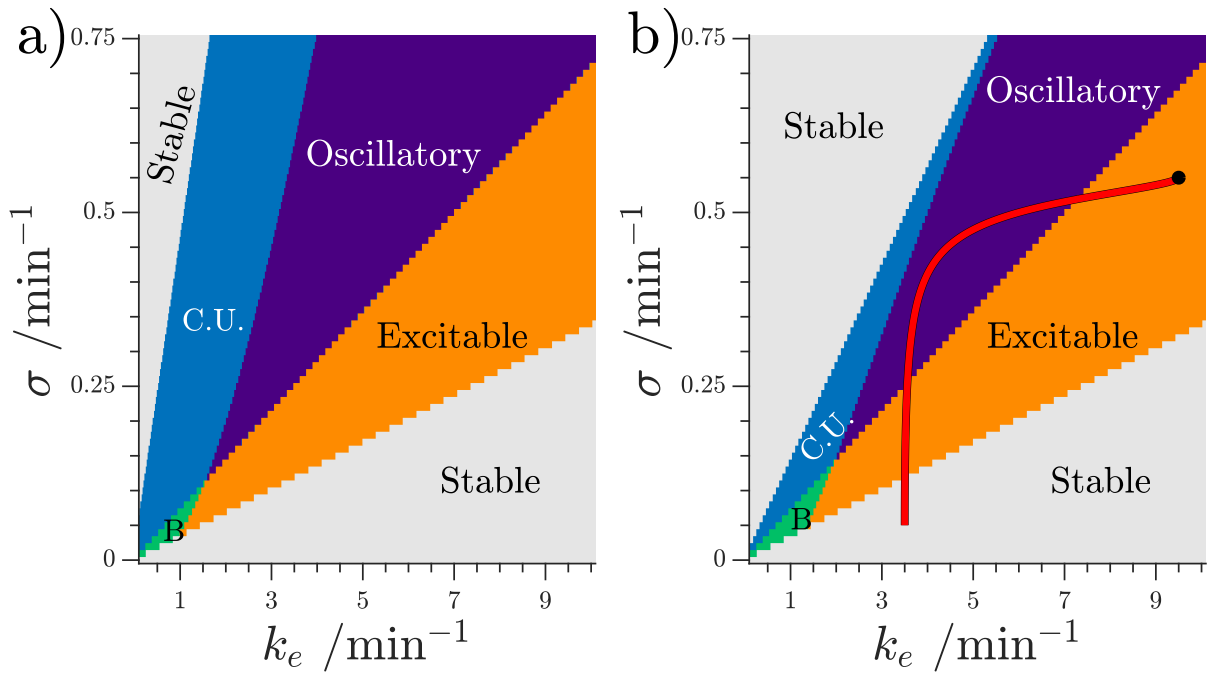

Figure 1.9: Phase diagram showing the different regimes in the Martiel-Goldbeter model with advection (Equation 1.7). a) 2-Component model. b) 3-Component model also showing the path taken by the cells in the developmental path scheme used in Chapter 3 (Equation 1.6). Cells start in the stable regime and end in the excitable, at the parameters marked by the black circle. Reproduced with minor modifications from [76].

The first big extension to this model was introduced by Tyson et al. in 1989 [77] who included diffusion of cAMP and showed the existence of planar waves in 1-D and concentric circular waves and spiral waves in 2-D. The model, therefore, takes the form

$$
\begin{aligned}
\partial_{t} \gamma & =k_{t} \beta / h-k_{e} \gamma+D \nabla^{2} \gamma, \\
\partial_{t} \beta & =q \sigma \alpha \Phi(\rho, \gamma) /(1+\alpha)-\left(k_{i}+k_{t}\right) \beta, \\
k_{1}^{-1} \partial_{t} \rho & =-f_{1}(\gamma) \rho+f_{2}(\gamma)(1-\rho),
\end{aligned}
$$


where $D$ is the diffusion coefficient of cAMP, and $\nabla^{2}=\partial_{x}^{2}+\partial_{y}^{2}$ is the Laplacian operator in 2-D or $\nabla^{2}=\partial_{x}^{2}$ in 1-D. In their description the system was analyzed in the excitable regime, where wave propagation occurs as follows. Independent of initial conditions the system relaxes back to its steady state solution. When a perturbation big enough is applied (suprathreshold perturbation), the perturbed area reacts producing a pulse of cAMP, which then diffuses and excites the neighboring cells, which in turn produce their own cAMP. This process, known as wave relay, produces two peaks traveling in opposite directions (away from the perturbation) in 1-D or a circular wave in 2-D.

A spiral wave is a solution that persists as a rotating spiral, emitting waves to the system. This solution can be created perturbing the system twice, if the second perturbation creates a wave such that a part of it is in an area where the cells are mostly refractory. The refractory cells are incapable of reacting to the perturbation and the wave front breaks. The open wave front curves and becomes the center of the spiral. Once the spiral has been created, it is a stable solution of the system, requiring no further perturbations to exist.

If the system is in the oscillatory regime, a perturbation creates concentric circular waves which are taken over by the synchronized homogeneous oscillation of the system, known as bulk oscillation. In this description the equations are homogeneous in space, that is, the system is invariant to a translation transformation $\mathbf{x} \rightarrow \mathbf{x}+\mathbf{r}$ with $\mathbf{r} \in \mathbb{R}^{2}$. Given these conditions different approaches have been used to break the homogeneity of the system and thus create spirals and target patterns in a persistent way. We will mention some of them here.

A rigorous deduction of the CGL Equation (Equation 1.2) starting on the oscillatory regime of the Martiel-Goldbeter model is included as an Appendix to this thesis, along with some comparisons of its predictions with the numerical simulations of the system. A comprehensive study of the predictions of the CGL equation for the system under study is beyond the scope of this work.

\subsubsection{Breaking Translational Invariance}

Lauzeral et al. [78] showed that by changing the system's parameters over time they could create persistent spirals in the system. The idea is based on experimental studies which show that ACA and phosphodiesterase activity in D. discoideum changes during starvation [79-81]. Their proposed model takes the cells along a developmental path which simultaneously increases $\sigma$ and $k_{e}$, following the relations

$$
\sigma(t)=0.3+0.25 \tanh \left(\frac{t-200}{50}\right) \text { and } k_{e}(t)=6.5+3 \tanh \left(\frac{t-260}{30}\right) .
$$

This path changes the system as time increases from the stable regime to the excitable regime, then the oscillatory, and finishes in the excitable regime, as shown in the phase 

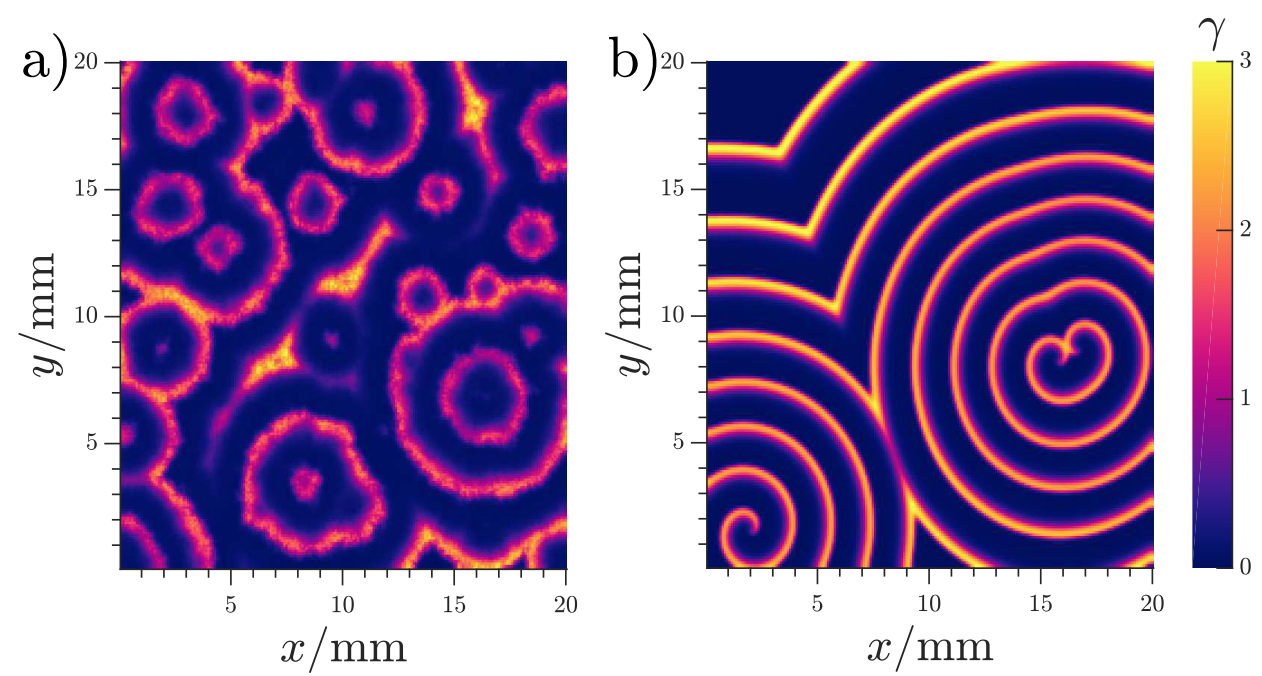

Figure 1.10: Typical structures appearing during D. discoideum signaling, simulated using the model described by Equation 1.5 and Equation 1.6. a) Multiple circular waves. b) Spiral waves, on the bottom left a single armed spiral can be observed and on the right side there are two connected single-armed spirals.

diagram of Figure $1.9 \mathrm{~b}$ ). To introduce inhomogeneity in the system, they divided their space in patches of $0.1 \mathrm{~mm} \times 0.1 \mathrm{~mm}$ and assigned a starting time for each patch. This starting time defines how advanced along the developmental path each patch starts. This inhomogeneity causes that different areas of the system change regime at different times, thus breaking the symmetric propagation of waves. Since at the end of this developmental path all cells end up with the same parameters, the system ends in the excitable regime in order for the spirals to persist. Structures produced following this method can be observed in Figure 1.10. It has been shown $[82,83]$ that in this description the cells who start more advanced in the developmental path and thus enter the oscillatory regime before the others, become target centers if they are sufficiently separated from one another. As cells progress in the developmental path the wave fronts from different centers break producing spirals. Therefore, spiral locations show anti-correlation with target center locations.

Other method for spiral creation in the excitable regime of this model was presented by Palsson and Cox [84], who showed that adding random firing to the cells, pairs of connected one-arm spirals appear (see Figure 1.10 b)). In their description, each cell had a probability $p$ of spontaneously emitting a cAMP pulse. They showed that if a pulse creates a wave that propagates into an area where the cells are mostly refractory (low $\rho$ ) the wave front can break, then both ends curve forming two connected spirals. They further argue that one of the spirals can take over the other one if the cells have an inhomogeneous phosphodiesterase $\left(k_{e}\right)$ distribution. In this way the spiral tip subjected to lower $k_{e}$ would rotate faster and eventually take over the slower rotating spiral.

Forcing one preferred direction is another way of breaking the translational symmetry 
of this system. In this regard, the work of Lindner et al. [85] studied the effect of adding an electric field to the cAMP signaling system. Since the cAMP molecules are negatively charged, applying a constant field moves the molecules along the electric field axis. This field affects the frequency and velocity of the propagating pulses in the excitable regime, and it was shown to be capable of destabilizing spirals.

Similar equations but in a different set of parameters were studied in the work of Gholami et al. [86, 87] where the effects of applying a constant flow to the external media were studied. The Martiel-Goldbeter model under the effect of advection is

$$
\begin{aligned}
\partial_{t} \gamma & =k_{t} \beta / h-k_{e} \gamma+D \nabla^{2} \gamma-V \cdot \nabla \gamma \\
\partial_{t} \beta & =q \sigma \alpha \Phi(\rho, \gamma) /(1+\alpha)-\left(k_{i}+k_{t}\right) \beta, \\
k_{1}^{-1} \partial_{t} \rho & =-f_{1}(\gamma) \rho+f_{2}(\gamma)(1-\rho),
\end{aligned}
$$

where $V$ is the velocity of the applied flow. For these equations the existence of a new regime in the parameter space, the convectively unstable regime was shown (see phase diagram, Figure 1.9). In this regime, for each set of parameters exists a minimum velocity such that the system becomes convectively unstable. If a perturbation is applied, it will either decay or increase depending on the applied flow. If the applied flow is smaller than the minimum velocity the perturbation will decay, but if it is higher than the threshold, the perturbation will increase in a moving reference frame, producing wave trains that are advected downstream. Chapter 2 of this thesis provides a full characterization and description of this regime, while Chapter 3 uses these equations to study the effect of high speed advecting flows in pattern formation in D. discoideum.

Patterns under the effect of advection similar to those studied in D. discoideum have also been observed in the Belousov-Zhabotinsky reaction where the application of an electric field to the system has been studied in different setups. In a tubular reactor (quasi 1-D experiments) it has been shown that a positive electric field applied in the wave traveling direction increases the wave velocity in a nonlinear manner and a negative field can stop or even split the traveling wave [88, 89]. In 2-D experiments it has been shown that an electric field can deform spirals [90] and drift their position towards the anode [91]. Experiments with flow have also been reported. In a tube reactor Agladze et al. [92] found that not only a maximum flow velocity exists that would prevent the propagation of waves against the flow, but also that at very high flow velocities wave propagation in the flow direction is also inhibited. It has also been shown that a flow can destabilize the steady state in the BZ reaction both in experiments [93] and in numerical simulations [94]. 

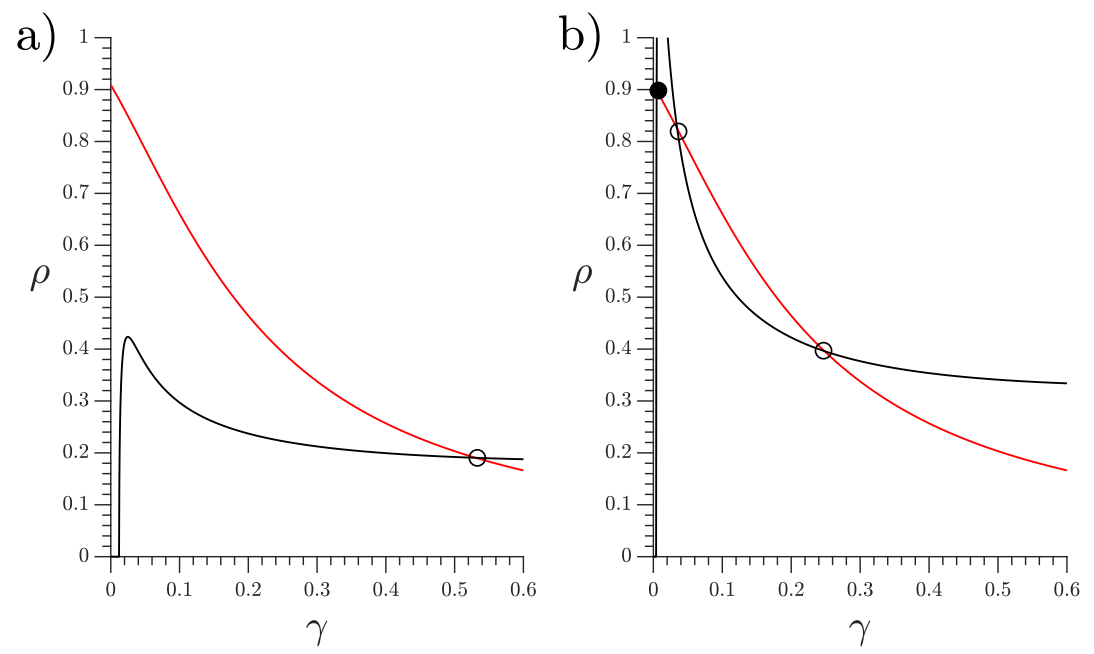

Figure 1.11: Nullclines for the 2-Component Martiel-Goldbeter model. $\partial_{t} \gamma=0$ in black and $\partial_{t} \rho=0$ in red. a) One solution at $k_{e}=4.0 \mathrm{~min}^{-1}$. b) Three solutions at $k_{e}=12 \mathrm{~min}^{-1}$.

\subsubsection{2-Component Martiel-Goldbeter Model}

In the final part of their work, Martiel and Goldbeter further reduced their system to just two equations, by making the transport of cAMP from the intracellular to the extracellular media instantaneous. Therefore, by taking $\partial_{t} \beta=0$ the system further reduces to

$$
\begin{aligned}
\partial_{t} \gamma & =s \Phi(\rho, \gamma)-k_{e} \gamma+D \nabla^{2} \gamma, \\
k_{1}^{-1} \partial_{t} \rho & =-f_{1}(\gamma) \rho+f_{2}(\gamma)(1-\rho),
\end{aligned}
$$

where $s=k_{t} q \sigma \alpha /\left[h(1+\alpha)\left(k_{i}+k_{t}\right)\right]$. They argued that while the experimental values of $k_{i}$ and $k_{t}$ (the cAMP degradation in the intracellular media and the transport rate respectively) do not justify the quasi steady state assumption; the 2-Component system behaves in a very similar way to the 3-Component one, showing both an oscillatory and an excitable regime, as can be seen in Figure 1.9 where the two phase diagrams are shown side by side for easy comparison. The advantage of this further reduction is that it is easier to analyze a system of two equations than one of three. For example, for the 2-Component model the number of solutions can be easily observed as the amount of times in which the nullclines (zero isoclines) cross, see Figure 1.11, and the eigenvalues of the Jacobian can be analytically calculated, thus simplifying the stability analysis. The analysis of the convective instability in Chapter 2 takes advantage of this description [95]. In Chapter 3 a comparison between the waves exhibited by the 2- and 3-Component models is presented [76]. 


\subsubsection{Modifications to the Model in the Presence of Caffeine}

To account for the reduction in cAMP production due to caffeine added to the experimental setup in Chapter 4 some parameters had to be modified. We initially chose to modify the parameter $K_{m}$ which corresponds to the Michelis constant of the reaction in which cAMP is produced from ATP, which means that it is an inverse measurement of the affinity between enzyme (ACA) and substrate (ATP). In the original paper by Martiel and Goldbeter $K_{m}$ appears indirectly as an adimensionalization factor affecting the parameters $q, \sigma$, and $\alpha$. Due to the way in which $q$ and $\sigma$ appear in the equations, the effect of modifying $K_{m}$ cancels out. Therefore, the net effect of modifying the Michelis constant $K_{m}$ is a change in the amount of ATP available for cAMP production. It follows the relation

$$
\alpha=[\mathrm{ATP}] / K_{m},
$$

where $[\mathrm{ATP}]$ is the intracellular ATP concentration, which is has been shown [75] not to change considerably during the course of one period in this model. For the simulations in Chapter 4 the parameter $K_{m}$ was modified between $0.4 \mathrm{mM}$ and $0.6 \mathrm{mM}$ corresponding to modify $\alpha$ between 3 and 2 . The effects of this modification on the system and how well it reproduces the effects of caffeine in the experimental system are calculated and discussed in Chapter 4 and in the Discussion.

Other possibility to account for the effects of caffeine was also explored at the end of Chapter 4. According to recent studies on the effects of caffeine [37] decreasing the ratio of activation of ACA would be a proper way of including the effects of caffeine into the numerical simulations. To accomplish this we modified the parameter $\epsilon$ which accounts for the reaction rate at which ACA is activated by the cAR1-cAMP complex. In the Martiel-Goldbeter model this reaction is represented as

$$
2 \mathrm{RP}+\mathrm{C} \underset{d_{3}}{\stackrel{a_{3}}{\rightleftarrows}} \mathrm{E},
$$

where RP is the cAR1-cAMP complex, $\mathrm{C}$ is the free form of ACA, and $\mathrm{E}$ is the activated form of the enzyme. Using the nomenclature of this reaction, $\epsilon \propto a_{3} / d_{3}$ where $a_{3}$ and $d_{3}$ are the forward and backward reaction rate constants, respectively. Since $\mathrm{C}$ and $\mathrm{E}$ are considered to be in a time independent steady state, $\mathrm{E} \propto \epsilon \mathrm{C}$. In the unmodified equation $\epsilon$ is chosen to be equal to 1 . To account for the effects of caffeine $\epsilon$ was varied between 0.7 and 1 . This modification showed similar results to modifying $K_{m}$ to reproduce the effects of caffeine. These results were included in the Supplementary Material of Chapter 4.

\subsection{Other D. discoideum Model}

As a comparison to the Martiel-Goldbeter model here is presented another approach that historically has been used to describe D.discoideum along with its main characteristics. 


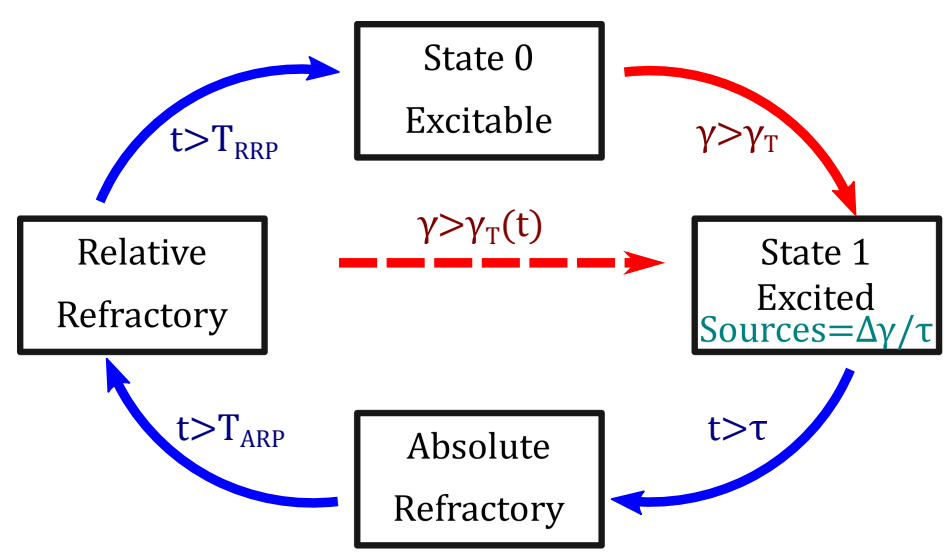

Figure 1.12: States of the Kessler-Levine Model. Transitions after a fixed time in the respective state are marked in blue, while transitions due to external cAMP concentration are marked in red. For all states the term sources in Equation 1.9 is 0, except for the excited State 1.

\subsubsection{Kessler-Levine Model}

This model was introduced by David A. Kessler and Herbert Levine in 1993 [96] with the goal of simplifying the biological process undergoing in the intracellular media, in order to focus on the physics of pattern formation. In their proposed model the only field variable is $\gamma$ which represents the extracellular cAMP concentration and is governed by the equation (names of variables changed to make them consistent with previous section)

$$
\frac{\partial \gamma}{\partial t}=D \nabla^{2} \gamma-k_{e} \gamma+(\text { sources })
$$

where $k_{e}$ is the degradation rate due to the action of phosphodiesterase, $D$ is the diffusion coefficient, and sources account for the cAMP production of the amoebas. This last term is the one that gives the nonlinearity to the system. To describe the production process each cell is replaced by a bion, a simple element of a cellular automata with 3 possible states. In state 0 the cells are in a steady excitable state where they do not produce cAMP. If the cell detects a cAMP concentration above threshold $\left(\gamma>\gamma_{T}\right)$ it changes to state 1 , the excited state. Then, the bion remains in this state during a time $\tau$ during which it secretes cAMP at a constant rate $\Delta \gamma / \tau$. Afterwards, the cell enters state 2 , the refractory state, in which it does not produce cAMP nor can be excited. The cell remains in this state for a time $t_{R}$ before finally coming back to state 0 and start the cycle again.

Kessler and Levine showed that their model is capable of sustaining a rotating spiral, after it is set up through initial conditions, and of relaying a circular target pattern which originates from a central pacemaker. This central pacemaker does not follow the bion state transitions, but simply emits a pulse with a fixed frequency.

This model was expanded to include a variable excitability by Levine et al [97, 98]. There, the refractory period (state 2 in the previous description) was divided in two 
parts: an absolute refractory period (ARP) and a relative refractory period (RRP). The ARP behaves as before, the cell spends a fixed amount of time $T_{A R P}$ in this state and cannot be excited. After the ARP, the cell enters the RRP, where it can be excited, that is, changed to state 1 if the cAMP concentration is bigger than a threshold $\gamma_{T}\left(t^{*}\right)$. If the cell is not excited in a time $T_{R R P}$, it returns to the excitable state 0 , see Figure 1.12 for a schematic representation of these transitions. The difference between the excitable state 0 , and the relative refractory state is the variable threshold for the excitation, which is given in the RRP by

$$
\gamma_{T}\left(t^{*}\right)=\left[\gamma_{\max }-A \frac{t^{*}}{t^{*}+T_{A R P}}\right](1-E),
$$

where $t^{*}$ is the time spent in the RRP, $A$ is a fixed parameter, and $E$ is the system's excitability. This excitability is a new field used to break the system's homogeneity according to the development of each cell, given by their exposure to cAMP waves. The excitability of each bion is described by the equation

$$
\frac{d E}{d t}=-\alpha E+\beta \gamma
$$

where $\beta$ provides a positive feedback between the passing of a cAMP wave and cell development. An upper limit $E_{\max }$ is set such that excitability can not increase above that value. To generate spirals in this description initially some randomly selected cells fire cAMP periodically (not following the previously described state transitions), acting as pacemakers. Since the surrounding cells have initially a low excitability these pulses fail to generate propagating waves. As the excitability of the system increases, the waves start to propagate further, but since not all cells have the same excitability level, the waves fail to propagate in low excitability areas, producing waves with open ends which curve and produce spirals; thus providing a mechanism for the creation of spirals.

The location of spirals appearing in this model was studied by Geberth and Hütt [99] who selected the firing cells randomly, but kept them fixed during the whole simulation (in contrast to the work of Sawai et al. [100] where each cell had a probability of firing). Geberth and Hütt showed that there is anti-correlation between the location of the firing cells (pacemakers) and the spiral tips. This is a direct consequence of increasing excitability with the passing of cAMP waves. The areas surrounding pacemakers increase their excitability with each passing pulse, allowing the wavefront to propagate, while the areas farther away from pacemakers maintain a lower excitability. It is in this low excitability areas, which are as far away as possible from pacemakers, where the wave front can not propagate and thus breaks, forming a spiral tip. Since there is little meandering in this model, that is, the spiral wave tips do not move far away from their original locations, the spiral tips end up located far away from pacemakers, 
thus showing anti-correlation. This is similar, but simpler than the findings of the same group for the Martiel-Goldbeter model with developmental path [82]. For the MartielGoldbeter model they found that spirals appear mostly as connected counter rotating pairs with their center of mass located in the areas where waves coming from different target centers collide. In other words, spirals are located at a finite distance from the Voronoid diagram produced by the target centers. In the Kessler-Levine model most firing cells become target centers, making the prediction of the spiral location much more straightforward than in the Martiel-Goldbeter model where the appearance of target centers not only depends on the development state of the cells, but also on their relative distance to other firing centers. Once the target centers have been established they are anti-correlated with the spiral locations. The statistical study carried out in that work [99] also predicts a higher spiral density for higher feedback strength $\beta$ in the Kessler-Levine model.

As a final remark, we would like to point out that the traveling waves in this model present an anomalous dispersion relation [63] which depends heavily on the parameter $T_{R R P}$. That means that the velocity-period curve of the wave front is not monotonically increasing as it is in many excitable systems (compare Figure 1.8 a) and b) to see the difference between the dispersion relation in this model and a normal one). It has also been shown [63] that the spirals in this model present a core instability if the refractory period $T_{R R P}$ is bigger than the rotation period of the spiral. This instability leads to a complex meandering motion of the spiral tip, that has not been observed in experiments.

\subsection{Motility Models}

Some of the first attempts at adding cell distribution to signaling models of $D$. discoideum are due to Höfer et al. [101, 102], who, based on the work of Keller and Segel [103], proposed to model the cell density $n$ as

$$
\partial_{t} n=\nabla \cdot(\mu \nabla n-\chi n \nabla \gamma)
$$

where $\mu$ is the cell diffusion coefficient, $\chi$ is the chemotactic coefficient, and $\gamma$ the cAMP concentration. Therefore, the first term on the right side of Equation 1.10 accounts for cell's natural random motion which is independent from cAMP; while the second term accounts for chemotaxis.

One task that this model and every model describing movement in D. discoideum needs to take into account is the so-called back of the wave paradox [104]. If the amoebas depended only on the cAMP gradient to decide their direction of motion, then their net movement would be very small, since they would move in one direction during the first half of the passing wave, and move back during the second half. This is of particular 
importance in this system because the cAMP wave is almost symmetrical in shape. In nature the cells move only in the first half of the wave [104] and even continue moving in that direction if the wave period is too short [5]. To account for such movement in this model a nonlinear chemotactic coefficient $\chi$ was introduced, such that

$$
\chi(\rho)=\chi_{0} \frac{\rho^{m}}{A^{m}+\rho^{m}},
$$

where $\chi_{0}, A$, and $m$ are positive constants, and $\rho$ is the fraction of active receptors in the cell. The other equations read

$$
\begin{aligned}
& \partial_{t} \rho=-k_{+} \gamma \rho+k_{-}(1-\rho), \\
& \partial_{t} \gamma=\lambda\left\{\phi(n) g_{+}(\rho, \gamma)-[\phi(n)+\delta] k_{e} \gamma\right\}+D \nabla^{2} \gamma
\end{aligned}
$$

where $g_{+}$is a nonlinear function that accounts for cAMP production and $\phi(n)$ accounts for local cell density effects. In this way, the density feeds-back to the cAMP production through this increasing function $\phi(n)$ which multiplies the production and degradation factors. Using this simplified version of the Martiel-Goldbeter model (Equations 1.11 coupled with Equation 1.10), Höfer et al. [101, 102] showed that their model produces aggregation streams. It is noteworthy that they added a minimum value for the degradation rate, ( $\delta$ in Equation 1.11 b)), therefore there is always phosphodiesterase activity even when the cell density is zero, thus taking into account unbounded phosphodiesterase. They also showed that no direct interaction between cells was necessary, in other words, they concluded that cell-cell adhesion was not necessary for stream formation. They also showed that a constant random walking speed (independent of density, that is, $\mu$ independent of $n$ in Equation 1.10) was enough to show streams. They explained stream generation as a patterning instability which is perpendicular to the direction of the cAMP wave.

In a later work by Vasiev et al. [105] they used a FitzHugh-Nagumo type of equation coupled with a density field to study stream formation. They made the production and degradation proportional to cell density and showed that the key factor in stream formation is the wave velocity dependence on density. That is, it is necessary that the cAMP wave travels faster in a higher density area than in a lower density area. This velocity difference is of great importance since it speeds up the wave front on different sections thus producing the wave instability described by Höfer. They argued this point by taking the limit case of making cAMP production independent of cell density, a condition in which wave speed is density independent. In this limit case streams did not form. It is again noteworthy that in this work cell-cell adhesion was again not considered, and therefore shown not to be strictly necessary for stream formation. The back of wave paradox was solved by taking a nonlinear chemotactic coefficient that 
allowed cells to move only if they were not in a refractory state.

A noteworthy attempt to include motility to the full Martiel-Goldbeter model is due to Van Oss et al. [106]. They used a cellular automata-like model, where cells were discretized in a square grid. If a square had an amoeba located in it, it would follow the Martiel-Goldbeter model, and if the square was empty of cells the system would simply diffuse the cAMP. Since the density is not a continuous variable in this description the back of the wave problem is solved by using the percentage of active receptors as an indicator of cell refractoriness. Therefore, the cells would move if they fulfilled two conditions, first that the cAMP gradient $|\nabla \gamma|$ was bigger than a threshold (to avoid movement due to noise), and second that the percentage of active receptors $\rho$ was higher than a certain threshold. The cells would then move with a constant velocity as long as these two conditions were fulfilled. It was shown that this model would not present streams in the 3-Component form of the model, but that it does show streams in the 2-Component form. Again, the argument boils down to the wave speed dependency on density. In their description, the wave speed was almost independent on density in the 3-Component version, but it showed a strong dependency in the 2-Component version. It is shown in Chapter 3 [76] of this work that using the 2Component model description leads to wave shapes that do not match the experimental observations, specially in situations where the fast cell reaction is of importance, like when fast advecting flows are used. The reconciliation of the 3-Component MartielGoldbeter model with streaming is presented in Chapter 4 and 5 [76, 107], the key solution is the inclusion of unbounded phosphodiesterase which degrades cAMP even in locations where cells are not present. This is different to the work of Van Oss where the cAMP would not be degraded by phosphodiesterase on the empty grid spaces. This difference is enough to produce variable wave speed and streams in the 3-Component model, along with other interesting properties that are explored in Chapter 5 [107].

\subsection{Computational Methods}

All numerical simulations in 1-D and 2-D were performed using finite differences for the space discretization, and a Runge-Kutta scheme with Merson error approximation [108] and an adaptive time step for the time discretization.

\subsubsection{Finite Differences}

In the finite differences method the continuous fields are discretized in a matrix of size $N_{x} \times N_{y}$, where the space coordinates are discretized in points with distances $d x$ and $d y$ between them for the $x$ and $y$ directions, respectively. Therefore, the space coordinates are discretized as $x=i d x$ and $y=j d y$ with $i, j \in \mathbb{N}$, and the size of the system is $L_{x} \times L_{y}$ with $L_{x}=N_{x} d x$ and $L_{y}=N_{y} d y$. 
The second derivative is discretized using a second order central discretization defined as

$$
\partial_{x x} \gamma(x) \approx \frac{\gamma(x-h)-2 \gamma(x)+\gamma(x+h)}{h^{2}}
$$

with an error $\mathcal{O}\left(h^{2}\right)$ where $h$ is a small distance. In our description this becomes

$$
\partial_{x x} \gamma_{i, j} \approx \frac{\gamma_{i-1, j}-2 \gamma_{i, j}+\gamma_{i+1, j}}{d x^{2}} \text { and } \partial_{y y} \gamma_{i, j} \approx \frac{\gamma_{i, j-1}-2 \gamma_{i, j}+\gamma_{i, j+1}}{d y^{2}}
$$

therefore we use a three-point Laplacian in one-dimensional simulations, and a five-point Laplacian in two-dimensional ones.

This discretization of the diffusion operator is positive by definition, which means that it can not bring the discretized field to a negative value [109]. This was specially important in our system since the physical meaning of $\gamma$ is the concentration of the chemical cAMP and therefore it can not go below zero. In particular, the numerical values of $\gamma$ become very small in the simulations of Chapter 2 [95]. There, the system represents a channel with cells on the bottom which are subjected to advecting flows. The system has an absorbing boundary condition $(\gamma(x=0)=0)$ in the upstream boundary, which flushes cAMP downstream, producing cAMP depletion for lower values of $x$.

To maintain the cAMP concentration above zero when fast flows were applied, a positive discretization of the first derivative needed to be implemented. Since we know beforehand the direction of the flow (and it does not change over time) an upwind discretization can be used, i.e., an asymmetric discretization. The first order upwind discretization is

$$
\partial_{x} \gamma_{i, j} \approx \frac{\gamma_{i, j}-\gamma_{i-1, j}}{d x}
$$

analogously if the flow is going in the $-\hat{x}$ direction, the first order downwind discretization can be defined

$$
\partial_{x} \gamma_{i, j} \approx \frac{\gamma_{i+1, j}-\gamma_{i, j}}{d x}
$$

These discretizations can not bring the concentration of $\gamma$ below zero, but are unfortunately very dissipative, thus introducing too much error in the simulations. The error in these approximations is $\mathcal{O}(d x)$.

The next order approximation is the second order central discretization, which has an error of $\mathcal{O}\left(d x^{2}\right)$ and is defined as

$$
\partial_{x} \gamma_{i, j} \approx \frac{\gamma_{i+1, j}-\gamma_{i-1, j}}{2 d x}
$$

This discretization was enough to keep $\gamma$ above zero for low flow velocities, but $\gamma$ would become negative for higher flow values since this discretization introduces an oscillatory behavior [109]. Since, by definition, the first order discretization is the only 
linear discretization that ensures positivity [109] we used a nonlinear discretization to maintain the cAMP concentration above zero at all times.

Before introducing the nonlinear discretization used, we show here the third order upwind discretization

$$
\partial_{x} \gamma_{i, j} \approx \frac{1}{d x}\left(-\frac{\gamma_{i-2, j}}{6}+\gamma_{i-1, j}-\frac{\gamma_{i, j}}{2}-\frac{\gamma_{i+1, j}}{3}\right)
$$

which is, again, not positive, but more accurate than the first upwind one. We used a nonlinear discretization proposed by Koren [110] that uses third order upwind discretization most of the time, but that reduces to first order when the gradients are too big, thus avoiding oscillatory behavior. To quantify these gradients we introduce the function $\theta$ such that

$$
\theta_{i, j}=\frac{\gamma_{i, j}-\gamma_{i-1, j}}{\gamma_{i+1, j}-\gamma_{i, j}}
$$

therefore for a smooth function $\theta \approx 1$. We also introduce a nonlinear limiter function $\psi(\theta)$. The discretization can be rewritten as

$$
\partial_{x} \gamma_{i, j} \approx \frac{1}{d x}\left(\gamma_{i, j}-\gamma_{i-1, j}\right)\left(1+\frac{\psi\left(\theta_{i}\right)}{\theta_{i}}-\psi\left(\theta_{i-1}\right)\right)
$$

where the first order upwind discretization is recovered by taking $\psi(\theta)=0$, first order downwind by $\psi(\theta)=1$, and the third order upwind by taking $\psi(\theta)=1 / 3-\theta / 6$. Positivity is ensured if [109]

$$
1+\frac{\psi\left(\theta_{i}\right)}{\theta_{i}}-\psi\left(\theta_{i-1}\right) \geq 0,
$$

which is satisfied if the conditions

$$
0 \leq \psi(\theta) \leq 1, \quad 0 \leq \frac{\psi(\theta)}{\theta} \leq \mu \quad \forall \theta \in \mathbb{R}
$$

with $\mu$ a positive parameter, are fulfilled. Following the work of Koren [110] we take $\mu=1$. Therefore, we define our limit function as

$$
\psi(\theta)=\max \left(0, \min \left(\theta, \frac{1}{3}+\frac{\theta}{6}, 1\right)\right)
$$

or equivalently

$$
\psi(\theta)= \begin{cases}0 & \text { if } \theta \leq 0 \\ \theta & \text { if } \theta \leq 2 / 5 \\ 1 / 3+\theta / 6 & \text { if } \theta \leq 4 \\ 1 & \text { otherwise. }\end{cases}
$$


This function is continuous, satisfies the conditions of positivity given by Equation 1.13, behaves like a third order approximation when $\gamma$ is smooth $(\theta \approx 1)$, and like a first order one in extreme cases $(\theta<0 \vee \theta>4)$. A representation of this limiting function can be found in Figure 1.13. We therefore used the discretization defined by Equation 1.12 and 1.14 for all simulations in this thesis that have an advecting flow.

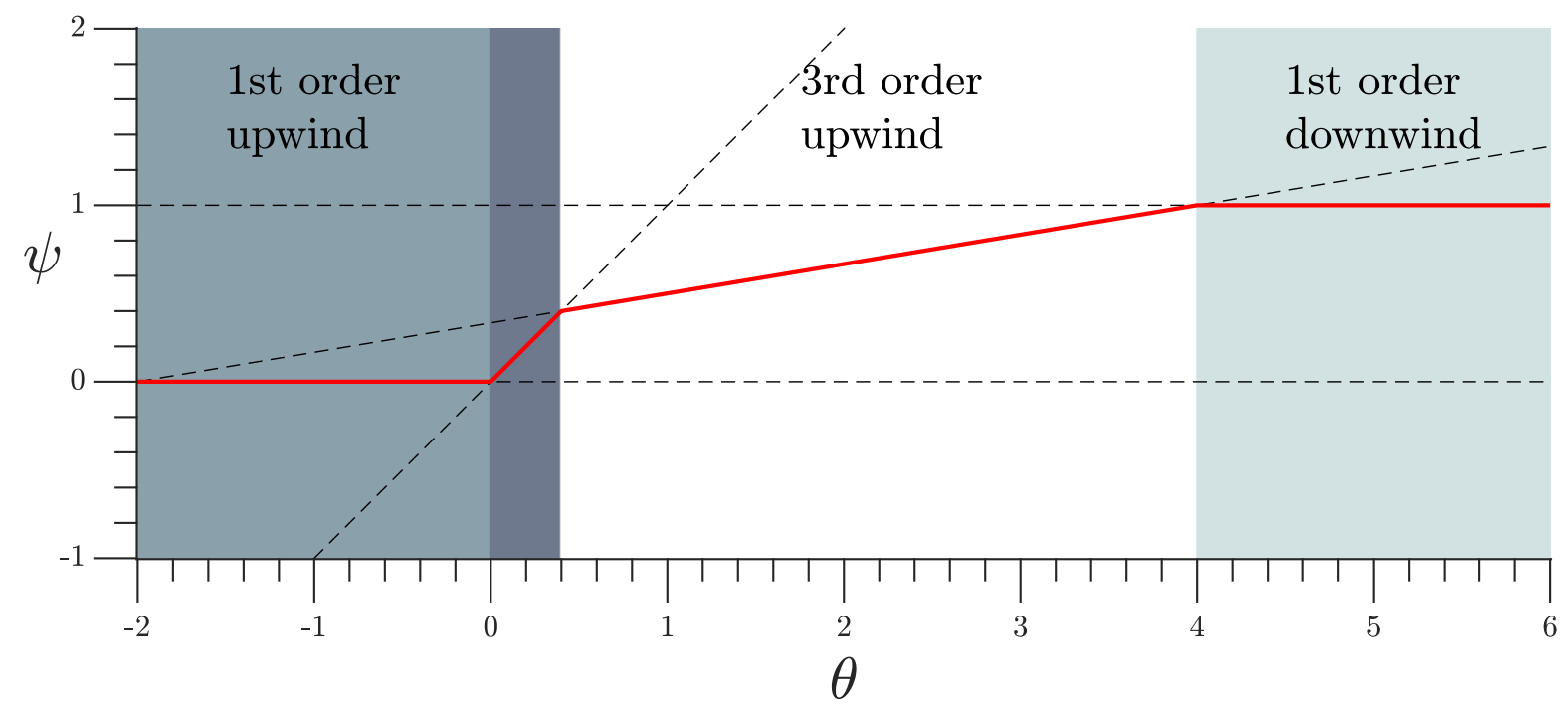

Figure 1.13: Limiting function $\psi$ used to discretize the advection operator. Used function $\psi=$ $\max (0, \min (\theta, 1 / 3+\theta / 6,1))$ in bold red line. Dashed lines indicating $\psi=0, \psi=\theta, \psi=1 / 3+\theta / 6$, and $\psi=1$ are drawn as reference. The values of $\theta$ at which the discretization function reduces to known linear discretizations are marked with color in the background.

\subsubsection{Runge-Kutta-Merson}

The time evolution of the system was performed using a Runge-Kutta scheme introduced by Merson [108], which is an explicit iterative method to calculate the time evolution of differential equations. For a differential equation given by

$$
\partial_{t} Y(x, y)=f(t, Y(x, y))
$$

at a given time step $i$, the intermediate steps $k_{1} \ldots k_{5}$ are calculated as

$$
\begin{aligned}
& k_{1}=h f\left(t_{i}, Y_{i}\right), \\
& k_{2}=h f\left(t_{i}+\frac{h}{3}, Y_{i}+\frac{k_{1}}{3}\right), \\
& k_{3}=h f\left(t_{i}+\frac{h}{3}, Y_{i}+\frac{k_{1}+k_{2}}{6}\right), \\
& k_{4}=h f\left(t_{i}+\frac{h}{2}, Y_{i}+\frac{k_{1}+3 k_{3}}{8}\right), \\
& k_{5}=h f\left(t_{i}+h, Y_{i}+\frac{k_{1}-3 k_{3}+4 k_{4}}{2}\right),
\end{aligned}
$$


where $h$ is the time step. The next iteration is then calculated as

$$
Y_{i+1}=Y_{i}+\frac{k_{1}+4 k_{4}+k_{5}}{6}
$$

and has an error estimation given by

$$
R \approx \frac{1}{30}\left(2 k_{1}-9 k_{3}+8 k_{4}-k_{5}\right)
$$

In our simulations $Y=(\gamma, \rho, \beta)$ and $f$ is given by the Martiel-Golbeter model, Equation 1.7. After each iteration the estimated error $R$ was calculated, if it exceeded the maximum error allowed, the time step $h$ was reduced in half, and the iteration was repeated until the error $R$ was under the allowed value.

\subsubsection{Adimensionalization}

The Martiel-Goldbeter model was not directly simulated as described in Equation 1.7, but instead space and time were first adimensionalized. Starting from Equation 1.7 we have

$$
\begin{aligned}
\partial_{t} \gamma & =k_{t} \beta / h-k_{e} \gamma+D \nabla^{2} \gamma-V \cdot \nabla \gamma, \\
\partial_{t} \beta & =q \sigma \alpha \Phi(\rho, \gamma) /(1+\alpha)-\left(k_{i}+k_{t}\right) \beta, \\
k_{1}^{-1} \partial_{t} \rho & =-f_{1}(\gamma) \rho+f_{2}(\gamma)(1-\rho),
\end{aligned}
$$

where the slow variable $\rho$ gives the timescale $k_{1}$, therefore we take the adimensional time $t^{\prime}=k_{1} t$. Since the length scale is given by the diffusion coefficient $D$, we make the space adimensional by taking $x^{\prime}=x k_{1} / \sqrt{k_{e} D}$. The system then becomes

$$
\begin{aligned}
\partial_{t^{\prime}} \gamma & =\frac{k_{t}}{h k_{1}} \beta-\frac{k_{e}}{k_{1}} \gamma+\frac{k_{1}}{k_{e}} \nabla^{\prime 2} \gamma-\frac{V}{\sqrt{k_{e} D}} \cdot \nabla^{\prime} \gamma, \\
\partial_{t^{\prime}} \beta & =\frac{q \sigma \alpha \Phi(\rho, \gamma)}{k_{1}(1+\alpha)}-\frac{k_{i}+k_{t}}{k_{1}} \beta, \\
\partial_{t^{\prime}} \rho & =-f_{1}(\gamma) \rho+f_{2}(\gamma)(1-\rho),
\end{aligned}
$$

which was the set of equations used to simulate the cAMP dynamics. 


\section{Convective Instability and Boundary Driven Oscillations}

The following paper originally appeared as Vidal-Henriquez, E., Zykov, V., Bodenschatz, E., \& Gholami, A. (2017). Convective instability and boundary driven oscillations in a reaction-diffusion-advection model. Chaos: An Interdisciplinary Journal of Nonlinear Science, 27(10), 103110. doi: 10.1063/1.4986153. It is licensed under CC BY 4.0.

EVH performed the numerical simulations and theoretical calculations. EVH wrote the manuscript. EVH and AG designed and planned the research. All authors reviewed and edited the manuscript. Figure 1 by AG, all other figures (2-13) by EVH. 


\title{
Convective instability and boundary driven oscillations in a reaction-diffusion-advection model
}

\author{
Estefania Vidal-Henriquez, ${ }^{1, a)}$ Vladimir Zykov, ${ }^{1}$ Eberhard Bodenschatz, ${ }^{1,2,3}$ \\ and Azam Gholami ${ }^{1, b)}$ \\ ${ }^{1}$ Max Planck Institute for Dynamics and Self-Organization, Am Fassberg 17, D-37077 Göttingen, Germany \\ ${ }^{2}$ Institute for Nonlinear Dynamics, University of Göttingen, D-37073 Göttingen, Germany \\ ${ }^{3}$ Laboratory of Atomic and Solid-State Physics and Sibley School of Mechanical and Aerospace Engineering, \\ Cornell University, Ithaca, New York 14853, USA
}

(Received 2 June 2017; accepted 25 September 2017; published online 9 October 2017)

\begin{abstract}
In a reaction-diffusion-advection system, with a convectively unstable regime, a perturbation creates a wave train that is advected downstream and eventually leaves the system. We show that the convective instability coexists with a local absolute instability when a fixed boundary condition upstream is imposed. This boundary induced instability acts as a continuous wave source, creating a local periodic excitation near the boundary, which initiates waves travelling both up and downstream. To confirm this, we performed analytical analysis and numerical simulations of a modified Martiel-Goldbeter reaction-diffusion model with the addition of an advection term. We provide a quantitative description of the wave packet appearing in the convectively unstable regime, which we found to be in excellent agreement with the numerical simulations. We characterize this new instability and show that in the limit of high advection speed, it is suppressed. This type of instability can be expected for reaction-diffusion systems that present both a convective instability and an excitable regime. In particular, it can be relevant to understand the signaling mechanism of the social amoeba Dictyostelium discoideum that may experience fluid flows in its natural habitat. (C) 2017 Author(s). All article content, except where otherwise noted, is licensed under a Creative Commons Attribution (CC BY) license (http://creativecommons.org/licenses/by/4.0/). https://doi.org/10.1063/1.4986153
\end{abstract}

In a reaction-diffusion-advection system, one or more species are carried away by a flowing medium with an externally imposed velocity. Therefore, the conditions of the system upstream become important to the phenomena observed downstream. In this work, we present the effects of adding an absorbing fixed boundary condition at the upstream end of the system. We focus on the convectively unstable regime, where a perturbation applied to the system dies out in the laboratory reference frame, while it grows in a moving one. By fixing the upstream boundary condition, the system becomes unstable, producing a trigger wave that travels upstream, and a wave train propagating downstream. The trigger wave is absorbed when it reaches the upstream boundary, then the system destabilizes again, and the phenomenon repeats. In 2-D simulations, the trigger wave propagating against the flow has a triangular shape, similar to the concentration profiles exhibiting a cusp in auto-catalytic advection reactions. ${ }^{1,2}$ The here reported mechanism can be expected to be applicable to other reaction-diffusionadvection systems in order to produce a continuous, periodic influx of wave trains.

\footnotetext{
${ }^{a)}$ Electronic mail: estefania.vidal@ds.mpg.de

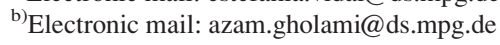

\section{INTRODUCTION}

Many out of equilibrium phenomena in nature can be described by reaction-diffusion systems. This includes the Belousov-Zhabotinsky reaction,, ${ }^{3,4}$ electrical impulse dynamics in the heart, ${ }^{5}$ skin patterns in fish, ${ }^{6}$ calcium dynamics in oocytes, ${ }^{7}$ and slime mold aggregation, ${ }^{8}$ among others. In many cases, the active components of such reactions might be subjected to advective flows, which cause new kinds of instabilities. ${ }^{9}$ The most commonly studied types of these instabilities are of convective or absolute nature. ${ }^{10,11}$ Both types of instabilities have been observed in simulations, 9,12 as well as in experiments such as the Belousov-Zhabotinsky reaction. ${ }^{13,14}$

Due to the advective nature of the flow, the upstream boundary conditions have important consequences for the spatio-temporal dynamics downstream. Most studies have been performed with no-flux boundary conditions or periodic boundaries, which simplifies the analysis by going into a comoving reference frame. Under these boundaries, an initial perturbation creates a growing wave train ${ }^{15,16}$ whose wavelengths and velocities depend on the particular characteristics of the system. However, the comoving frame analysis is impossible with a Dirichlet (fixed) boundary condition. In particular, an absorbing (zero amplitude) boundary condition corresponds to a one dimensional defect and is the one dimensional equivalent of a spiral center in excitable systems. ${ }^{17-19}$ Up to now, the effects of this type of upstream 
condition on an advection-diffusion system have received little attention. Preliminary results on such a system were presented by Gholami et al. ${ }^{20,21}$ where a continuous influx of wave trains was observed.

Here, we show that in the reaction-diffusion-advection system under study (see below), a particular kind of boundary induced instability occurs when the advection velocities are below a threshold. This boundary condition creates waves periodically with a period dependent on the imposed flow velocity. Unlike the commonly emitted waves by a boundary, these waves do not travel in just one direction (either towards or away from the boundary as is usual in these systems ${ }^{22}$ ), but instead two waves appear, one that travels towards and one that travels away from the boundary. In order for this to be possible, these waves do not grow directly at the boundary, but at a finite distance from it. The reaction of the system to this boundary driven instability is also different from the way it reacts to an external perturbation. In this system, a perturbation creates a growing wave train that is advected downstream, while in the absorbing boundary case, the growing instability produces not only a wave train downstream but also a wave travelling upstream.

The downstream wave train is equivalent to the one observed with the no-flux boundary condition. We fully characterized this wave train using linear stability analysis in a moving reference frame and calculated the periodic travelling wave solutions. The upstream travelling wave is the novel feature of this process. This wave travels upstream until it reaches the fixed boundary where it is absorbed, and the process starts again. This process creates wave trains with a period dependent on the imposed flow velocity and thus provides a mechanism to continuously generate wave trains in the fixed reference frame.

To investigate this effect, we performed numerical simulations in one dimension of a model proposed by Martiel and Goldbeter ${ }^{23}$ which are reaction-diffusion equations, with the addition of an advection term due to an imposed external flow. To ensure accuracy in the simulations, we implemented a Runge-Kutta scheme with an adaptable time step based on the Merson error estimation. ${ }^{24}$ To complete the study of the convectively unstable regime, we also performed linear stability analysis of the system in a moving reference frame and periodic travelling wave calculations which we compared with the full nonlinear system solutions. Finally, we performed numerical simulations in 2-Dimensions to study the effect of the flow profile on the boundary induced oscillations. Similar to fronts in advected auto-catalytic reactions, ${ }^{1,2}$ we observed a strong triangular deformation of the trigger wave travelling upstream.

\section{THE REACTION-DIFFUSION-ADVECTION MODEL}

Cellular slime moulds are unique organisms positioned between uni- and multi-cellular life in the evolutionary tree. The amoebae of the cellular slime mould Dictyostelium discoideum normally live as single cells in forest soil and feed on bacteria. They multiply by binary fission. Starvation induces a developmental program in which up to $10^{5}$ amoebae aggregate chemotactically to form a multicellular mass, ${ }^{25}$ the so-called slug, that behaves as a single organism and migrates to search for food and better environmental conditions. On failing to find nutrients, the slug culminates into a fruiting body consisting of a stalk and a mass of spores. ${ }^{26}$ Spores are dispersed by rain and small animals and under suitable conditions germinate to release amoebae and the whole cycle starts over again.

Cyclic adenosine monophosphate (cAMP) is the primary chemoattractant for the $D$. discoideum cells during early aggregation. cAMP is emitted from the aggregation centers in a pulsatile manner and surrounding cells detect it by highly specific cAMP receptors. ${ }^{27}$ When cAMP binds to the receptors, it triggers a series of intracellular reactions that activate an enzyme called Adenylate cyclase (ACA), which in turns consumes Adenosine triphosphate (ATP) to produce intracellular cAMP. The cAMP produced inside the cell is partially degraded by intracellular phosphodiesterase and partially transported to the extracellular medium. Phosphodiesterase secreted by the cells degrades extracellular cAMP and suppresses the accumulation of excessive cAMP in the aggregation field (Fig. 1). Since each cell

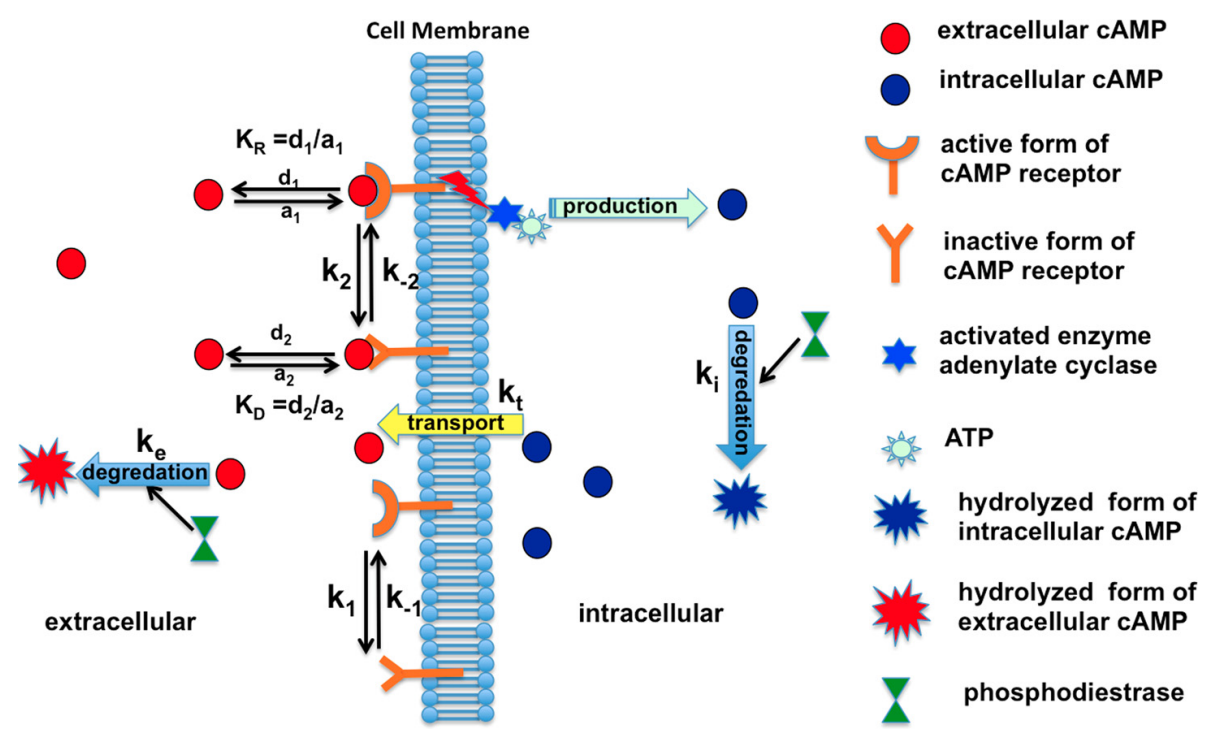

FIG. 1. Schematic representation of the model proposed by Martiel and Goldbeter for production and relay of cAMP in D. discoideum. The extracellular cAMP binds with the membrane receptors at a rate $K_{R}$ for those in the active state, activating ACA which in turn produces intracellular cAMP that is transported to the extracellular media at a rate $k_{t}$. After binding, the receptors change to their inactive form at a rate $k_{1}$ which has a lower probability of binding with cAMP $\left(K_{D}\right)$. The extracellular cAMP is then degraded via phosphodiestrase at a rate $k_{e}$. Reproduced from Ref. 20. 
responds to cAMP by moving towards the source of cAMP, by emitting a pulse of cAMP itself, and by a refractory period, the cAMP signal is relayed outward from the aggregation center as a wave. ${ }^{28,29}$ During the refractory period, the amoebas that have detected and produced cAMP do not react to it for a few minutes, and therefore, a new cAMP wave cannot pass during this period. This refractory phase is included in the model in terms of the membrane receptors. These receptors are present in two states, active and inactive. The first one has a higher probability to bind with cAMP than the second one. Once the receptors bind with cAMP, they change their state to inactive and then slowly change back to their active state. This combination of relay and refractory phase is characteristic of excitable systems and produces target patterns or spirals in two dimensional systems. The geometry of propagating waves is analogous to the spatio-temporal pattern of chemical waves in the Belousov-Zhabotinski reaction. ${ }^{3,4}$

The model we used for our study was initially proposed by Martiel and Goldbeter ${ }^{23}$ and extended by Tyson et al. $^{30}$ In this reaction-diffusion system, the concentration of the signalling chemical cAMP is the activator, while the cAMP receptors on the cell membrane act as inhibitors. Since the inhibitor is cell bounded and we assume that the imposed flow is not strong enough to detach the cells from the substrate, ${ }^{31}$ we add the advection term only to the activator dynamics. A detailed model derivation and the biological correspondence of the model parameters can be found in Ref. 23.

The main equations of the model in its three component version are as follows, where $\rho$ stands for the percentage of active receptors on the cell membrane, $\gamma$, the extracellular concentration of cAMP, and $\beta$, the intracellular amount of cAMP. The receptor dynamics are given by

$$
\partial_{t} \rho=-k_{1} f_{1}(\gamma) \rho+k_{1} f_{2}(\gamma)(1-\rho)
$$

with

$$
f_{1}(\gamma)=\frac{1+\kappa \gamma}{1+\gamma}, \quad f_{2}(\gamma)=\frac{\mathcal{L}_{1}+\kappa \mathcal{L}_{2} c \gamma}{1+c \gamma}
$$

where $f_{1}$ controls the receptor desensitization (change from active to inactive state) and $f_{2}$, the resensitization. The intracellular cAMP is increased by the cAMP production, which in turn depends on the extracellular cAMP and the active receptors. This production is tuned through the rate $\sigma$ at which the activated ACA produces cAMP. The intracellular cAMP is diminished through degradation by intracellular phosphodiesterase at a rate $k_{i}$ and passive transport outside of the cell at a rate $k_{t}$

$$
\partial_{t} \beta=q \sigma \alpha \Phi(\rho, \gamma) /(1+\alpha)-\left(k_{i}+k_{t}\right) \beta
$$

with

$$
\Phi(\rho, \gamma)=\frac{\lambda_{1}+Y^{2}}{\lambda_{2}+Y^{2}}, \quad Y=\frac{\rho \gamma}{1+\gamma},
$$

$\lambda_{2}=(1+\alpha \theta) /(\epsilon(1+\alpha))$, and $\lambda_{1}=\lambda \theta / \epsilon$. The extracellular concentration of cAMP $\gamma$ is degraded at a rate $k_{e}$ by the extracellular phosphodiesterase and is increased by the transport of cAMP from the intracellular medium

$$
\partial_{t} \gamma=D \nabla^{2} \gamma-v \cdot \nabla \gamma+k_{t} \beta / h-k_{e} \gamma
$$

We nondimensionalize the system by introducing dimensionless time and space as $t^{\prime}=t \cdot k_{1}$ and $x^{\prime}=x \cdot k_{1} / \sqrt{k_{e} D}$. Dropping primes and setting $\epsilon_{1}=k_{1} / k_{e}, \epsilon^{\prime}=k_{1} /\left(k_{i}+k_{t}\right)$, we arrive at

$$
\begin{gathered}
\partial_{t} \rho=-f_{1}(\gamma) \rho+f_{2}(\gamma)(1-\rho), \\
\epsilon^{\prime} \partial_{t} \beta=q \sigma \alpha \Phi(\rho, \gamma) /\left((1+\alpha)\left(k_{i}+k_{t}\right)\right)-\beta, \\
\partial_{t} \gamma=\epsilon_{1} \nabla^{2} \gamma-v \cdot \nabla \gamma+\left(k_{t} \beta /\left(h k_{e}\right)-\gamma\right) / \epsilon_{1} .
\end{gathered}
$$

Finally, we reduce this system to a two component model which simplifies its theoretical treatment. For this, we assume $\epsilon^{\prime}$ small, which means that the intracellular cAMP is instantaneously transmitted to the outside media (for a discussion on the validity of this approximation, refer to Refs. 23 and 30). We then arrive at the two component Martiel-Goldbeter, which we will use during the rest of this paper

$$
\begin{gathered}
\partial_{t} \gamma=\epsilon_{1} \nabla^{2} \gamma-v \cdot \nabla \gamma+(s \Phi(\rho, \gamma)-\gamma) / \epsilon_{1}, \\
\partial_{t} \rho=-f_{1}(\gamma) \rho+f_{2}(\gamma)(1-\rho),
\end{gathered}
$$

where $s=q k_{t} \alpha \sigma /\left(k_{e}\left(k_{t}+k_{i}\right) h(1+\alpha)\right)$. All used parameters are listed in Table I and were selected as suggested by Lauzeral et $a l .{ }^{32}$ because of their good agreement with experimental measurements. We selected $\sigma$ and $k_{e}$ as control parameters since they account for the production and degradation of extracellular cAMP, respectively. Depending on these two parameters, this system can have one, two, or three steady state solutions, as is shown in the phase diagram in Fig. 2. We focused on the range where only one steady state exists (green, yellow, and blue in Fig. 2). We performed linear stability analysis around this steady state solution $\left(\gamma_{0}, \rho_{0}\right)$ by setting $\gamma=\gamma_{0}+\gamma^{\prime}$, $\rho=\rho_{0}+\rho^{\prime}$, linearizing, dropping primes, and performing Fourier transform

$$
(\gamma, \rho)=\int_{-\infty}^{\infty}\left(\gamma_{k}, \rho_{k}\right) e^{\omega(k) t+i k x} d k,
$$

we arrive at the dispersion relation

$$
0=\omega^{2}+\omega\left(-T+\epsilon_{1} k^{2}+i v k\right)+\Delta-a_{22}\left(\epsilon_{1} k^{2}+i v k\right),
$$

where $\Delta=a_{11} a_{22}-a_{12} a_{21}, T=a_{11}+a_{22}$,

TABLE I. Parameters used for simulations of Eq. (2).

\begin{tabular}{lcc}
\hline \hline$c=10$ & $h=5$ & $k_{1}=0.09 \mathrm{~min}^{-1}$ \\
$k_{2}=1.665 \mathrm{~min}^{-1}$ & $K_{R}=10^{-7} \mathrm{M}$ & $k_{i}=1.7 \mathrm{~min}^{-1}$ \\
$k_{t}=0.9 \mathrm{~min}^{-1}$ & $\mathcal{L}_{1}=10$ & $\mathcal{L}_{2}=0.005$ \\
$q=4000$ & $\epsilon=1$ & $\lambda=0.01$ \\
$\theta=0.01$ & $\alpha=3$ & $D=0.024 \mathrm{~mm}^{2} \cdot \mathrm{min}^{-1}$ \\
\hline \hline
\end{tabular}




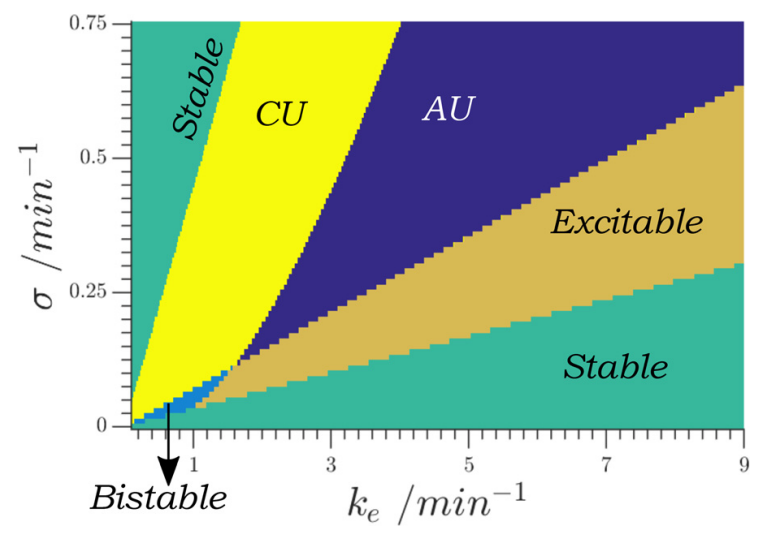

FIG. 2. Phase diagram of the system described by Eq. (2). Stable regime in green, in this regime only one solution exists and is stable. In the yellow area labeled CU exists one steady state that is convectively unstable. In the AU labeled blue area exists one unstable steady state surrounded by a limit cycle. The orange regime marked as excitable presents three steady states, one of which is excitable, while the light blue bistable regime has three steady states, two of which are stable.

$$
\begin{aligned}
& a_{11}=\frac{s}{\epsilon_{1}} \frac{2 \rho_{0}^{2} \gamma_{0}\left(\lambda_{2}-\lambda_{1}\right)}{\left(1+\gamma_{0}\right)^{3}\left(\lambda_{2}+Y_{0}^{2}\right)^{2}}-\frac{1}{\epsilon_{1}}, \\
& a_{12}=\frac{s}{\epsilon_{1}} \frac{2 \gamma_{0}^{2} \rho_{0}\left(\lambda_{2}-\lambda_{1}\right)}{\left(1+\gamma_{0}\right)^{2}\left(\lambda_{2}+Y_{0}^{2}\right)^{2}}, \\
& a_{21}=\left(1-\rho_{0}\right) \frac{\kappa \mathcal{L}_{2} c-c \mathcal{L}_{1}}{\left(1+c \gamma_{0}\right)^{2}}-\frac{\rho_{0}(\kappa-1)}{\left(1+\gamma_{0}\right)^{2}}, \text { and } \\
& a_{22}=-f_{1}\left(\gamma_{0}\right)-f_{2}\left(\gamma_{0}\right) .
\end{aligned}
$$

From here, the different regimes can be distinguished. Starting with the left green area of the phase diagram of Fig. 2 and with no imposed flow velocity $v=0$, the system has $T<0$ and $\Delta>0$; therefore, $\operatorname{Re}(\omega)<0$ for every $k$ and the system is stable. Increasing $k_{e}$, the system has a Hopf bifurcation (at the boundary between yellow and blue area in Fig. 2) and a limit cycle appears (Oscillatory regime). When $v \neq 0$, part of the stable regime becomes convectively unstable (yellow in Fig. 2). In this area, $a_{11}$ is positive and we can calculate the minimum imposed velocity at which the system becomes unstable, by calculating when the real part of $\omega$ becomes positive. This gives the following relation:

$$
v^{2}(k)=\frac{\left(-\Delta / a_{22}+\epsilon_{1} k^{2}\right)\left(\epsilon_{1} k^{2}-T\right)^{2}}{k^{2}\left(a_{11}-\epsilon_{1} k^{2}\right)} .
$$

This is a convex curve dependent on $k$ with asymptotes at $k=0$ and $k=\sqrt{a_{11} / \epsilon_{1}}$. Its global minimum corresponds to the critical velocity $v_{c}$ at which the system destabilizes. This type of instability is of the convective type, which means that although a perturbation applied to the system will die out in the laboratory reference frame, it will grow in a reference frame moving with a speed $v^{\prime}$, when the system is advected with a flow higher than $v_{c}$. All our simulations were performed in this regime.

Before proceeding to the characterization of the boundary driven instability, we perform a general description of the wave trains present in this system.

\section{NO-FLUX BOUNDARY CONDITION}

In the convectively unstable regime, when the advection velocity $v$ is above the critical value $v_{c}$ [calculated as the minimum of Eq. (4)], a perturbation creates a peak that is advected downstream. This peak creates further peaks behind it, producing a wave train, as can be observed in Fig. 3. The front of this wave train travels with a speed $v_{f}$ higher than the imposed flow $v$, while the rear of the wave train travels with a velocity $v_{b}<v$. This difference between $v_{b}$ and $v_{f}$ translates into the wave train growing in size and having more peaks as time passes. These velocities are indicated by colored lines in Fig. 3. The characteristics of these wave trains can be estimated by taking the Fourier transform in a moving reference frame $y=x-v^{\prime} t$, where $v^{\prime}$ is a free parameter

$$
(\gamma, \rho)=\int_{-\infty}^{\infty}\left(\gamma_{k}, \rho_{k}\right) e^{t(\omega+i k v \prime)+i k y} d k,
$$

with $k \in \mathbb{C}$ and $\omega(k)$ given by the dispersion relation, Eq. (3). According to the method of steepest descents, ${ }^{10}$ the long term behavior of this integral is given by the saddle point of the term accompanying $t$, i.e.,

$$
\frac{d}{d k}\left(\omega(k)+i k v^{\prime}\right)=0 .
$$

Since $\omega$ is also complex, we can use the Cauchy-Riemann Equations

$$
\frac{\partial \omega_{r}}{\partial k_{r}}=\frac{\partial \omega_{i}}{\partial k_{i}}=0 \quad \text { and } \quad \frac{\partial \omega_{r}}{\partial k_{i}}-v^{\prime}=\frac{\partial \omega_{i}}{\partial k_{r}}+v^{\prime}=0,
$$

where $k=k_{r}+i k_{i}$ and $\omega=\omega_{r}+i \omega_{i}$. This gives pairs of solutions $\left(k, v^{\prime}\right)$, each with its growing rate $\lambda_{r}=\omega_{r}-k_{i} v^{\prime}$. A typical curve $\lambda_{r}$ vs $v^{\prime}$ is shown in Fig. 4. The maximum of this curve corresponds to the group velocity of the wave train, it is the fastest growing mode and has $k_{i}=0, k_{r} \neq 0$. To calculate the edges of the wave train, the relevant values

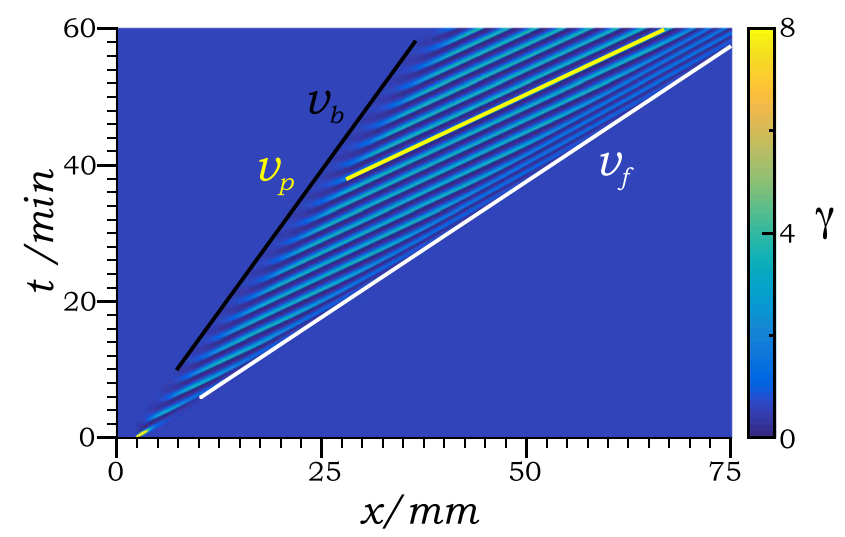

FIG. 3. Space-time plot of a simulation performed in the convectively unstable regime using no-flux (Neumann) boundary condition. The wave train is generated by an initial perturbation and is advected downstream (to the right) by the imposed flow. The relevant velocities present in the wave train are highlighted, these are the velocity of wave train rear $v_{b}$ in black, front velocity $v_{f}$ in white, and individual peak velocity $v_{p}$ in yellow. All numerical simulations were performed using $k_{e}=3.0 \mathrm{~min}^{-1}$ and $\sigma=0.45 \mathrm{~min}^{-1}$. 


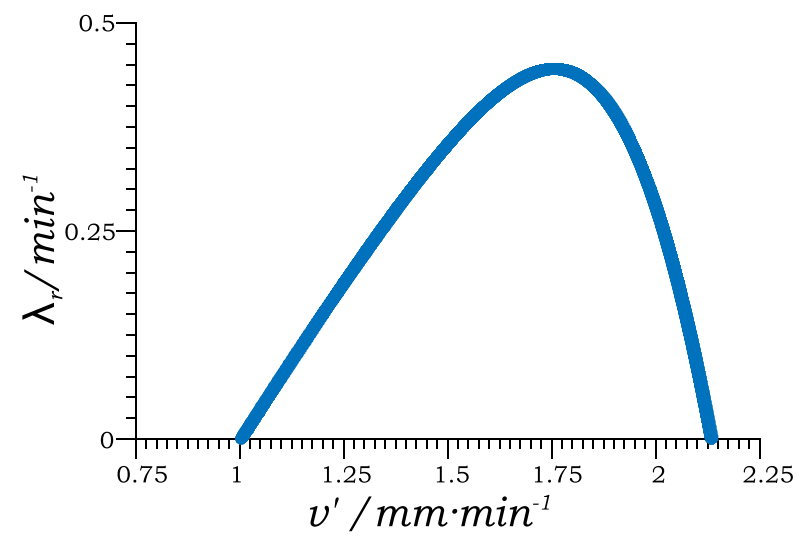

FIG. 4. Growth rate in the different reference systems $v^{\prime}$ for $v=2 \mathrm{~mm} / \mathrm{min}$. The intersections with the $x$-axis mark the back and front velocities of the wave train. For these parameters $v_{b}=1.00 \mathrm{~mm} / \mathrm{min}$ and $v_{f}=2.13 \mathrm{~mm} / \mathrm{min}$.

are the pairs with zero growing rate, because these will correspond to the first and last points at which the system destabilizes and therefore mark the boundaries of the velocity range at which the wave train can be observed. There are two velocities $v^{\prime}$ with zero growing rate, the lower corresponds to $v_{b}$ and the higher to $v_{f}$. This linear calculation has a very good agreement with the velocities calculated from the numerical simulations of the full nonlinear system, Eq. (2). This is shown in Fig. 5 where these two data sets are compared.

It has been shown that for some systems, the previously used method may not catch the fastest growing mode in a moving reference frame. ${ }^{33,34}$ For this, the more reliable Briggs collision criterion $^{35}$ is recommended. However, in our system the function $\omega(k)$ has only two local maxima and one unstable branch for real $k$, and under these conditions, the saddle point approach is enough to find all the unstable points. $^{36}$

To connect to other results in literature, it is worth mentioning that in our calculation, $v_{f}$ is equivalent to the spreading speed to the right of the system, ${ }^{37,38}$ which means that it

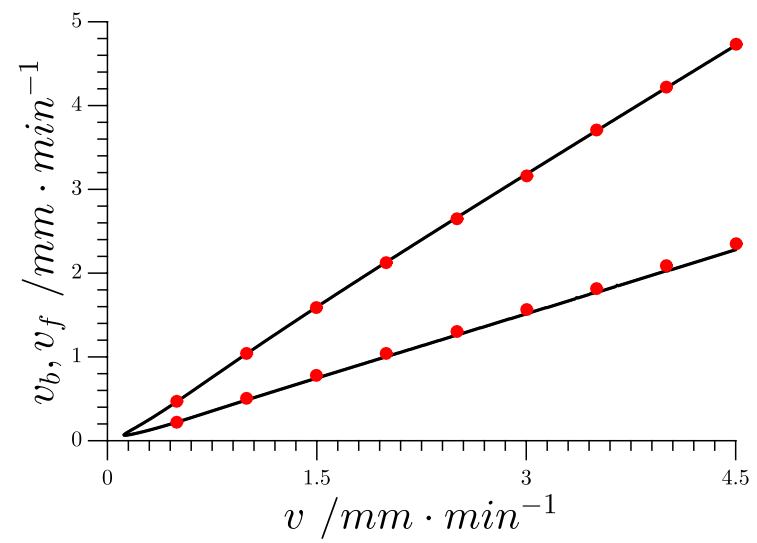

FIG. 5. Dependence of the wave train velocities on the imposed advection flow. The lower value corresponds to the back of the train, that is, the first point that destabilises, while the higher value corresponds to the front of the wave train, that is, the last point that destabilises. The continuous line corresponds to the prediction obtained by the linear analysis, and the dots are the values obtained from the simulations of the full nonlinear equations. is the supreme of the velocities $v^{\prime}$, such that the system is unstable in the comoving frame moving at $v^{\prime}$.

To characterize each individual peak velocity $v_{p}$, we studied the periodic travelling wave solutions of this system. These waves are characteristic of oscillatory systems ${ }^{39}$ and have the property $\gamma(z+T)=\gamma(z)$ with $z=x-c t$ for a certain combination of propagation velocity $c$ and period $T$. The wave calculation and stability analysis were performed using the software Wavetrain. ${ }^{40-42}$

We found a range of velocities $c$ at which the periodic travelling wave solutions exist. Inside this range, there is a band of velocities $c$ where they are stable. The velocities of each individual peak fall into this band as shown in Fig. 6. The selection of a particular wave solution depends on the initial conditions.

The velocity of each particular peak $v_{p}$ is higher than the front velocity; therefore, each peak moves forward in the train until it approaches the front, where it has to slow down until it matches $v_{f}$, the velocity of the front of the wave train. Since wavelength and velocity are uniquely linked, the peaks closer to the front of the wave train have a smaller wavelength than the rest of the train. This creates a traffic jam where more peaks start to accumulate in this shorter wavelength area at the front of the train. A similar process has been observed in other reaction-diffusion systems. ${ }^{43,44}$

\section{FIXED UPSTREAM BOUNDARY CONDITION}

We performed numerical simulations with a Dirichlet (fixed) boundary condition upstream $\gamma(x=0)=\rho(x=0)=0$ in the convectively unstable regime. We found that for very high flow speeds, the advection dominates over the diffusion and the system reaches a stable extended steady state. This state can be approximated in powers of $\delta=\epsilon_{1} / v^{2}$ with the time independent version of Eq. (2) as

$$
\begin{aligned}
\delta \partial_{x^{\prime} x^{\prime}} \gamma & =\partial_{x^{\prime} \gamma}-(s \Phi(\rho, \gamma)-\gamma) / \epsilon_{1}, \\
\rho & =f_{2}(\gamma) /\left(f_{1}(\gamma)+f_{2}(\gamma)\right),
\end{aligned}
$$

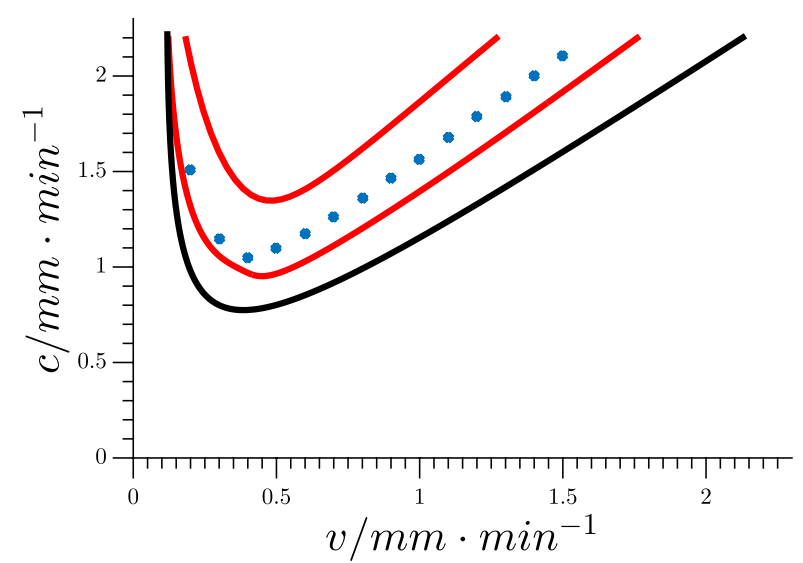

FIG. 6. Region of existence of periodic travelling wave solutions. $v$ stands for the imposed advection velocity, and $c$, the velocity of the periodic travelling wave. The solutions exist above the black line and are stable in the band between the red lines. The dots correspond to the solution selected by the system in the middle of the wave train in our numerical simulations. 
a)

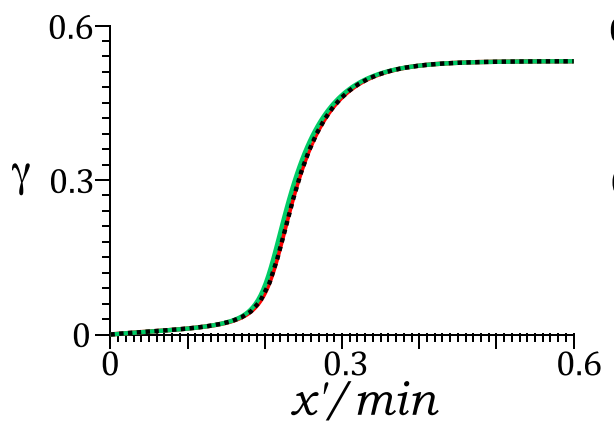

b)

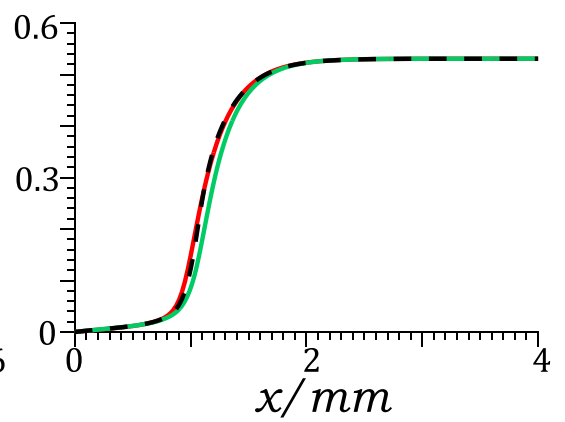

FIG. 7. (a) High speed solution with Dirichlet boundary condition. Advection velocity $v=2 \mathrm{~mm} / \mathrm{min}$ in green and $v=5 \mathrm{~mm} / \mathrm{min}$ in red. Zero order approximation $\varphi_{0}$ solution of Eq. (6a) in black dotted lined. Scaled space $x^{\prime}=x / v$. (b) Comparison for approximation at smaller speed. Full solution with Dirichlet boundary condition and $v=1.33 \mathrm{~mm} / \mathrm{min}$ in red. Zero order approximation $\varphi_{0}$ in green and first order approximation $\gamma=\varphi_{0}+\delta \varphi_{1}$ in dashed black line. where $x^{\prime}=x / v$ and $\gamma\left(x^{\prime}=0\right)=0$. The first two terms of the expansion were calculated taking $\gamma=\varphi_{0}+\delta \varphi_{1}$

$$
\begin{gathered}
0=\partial_{x^{\prime}} \varphi_{0}-\left(s \Phi\left(\varphi_{0}\right)-\varphi_{0}\right) / \epsilon_{1}, \\
\partial_{x^{\prime} x^{\prime}} \varphi_{0}=\partial_{x^{\prime}} \varphi_{1}-\varphi_{1}\left(\left.s \frac{d \Phi}{d \gamma}\right|_{\gamma=\varphi_{0}}-1\right) / \epsilon_{1} .
\end{gathered}
$$

This solution connects smoothly the zero boundary condition with the steady state of the system. This approximation matches quite well with the full solution as it is shown in Fig. 7.

We performed numerical linear analysis of this monotone solution and found that it becomes unstable at smaller velocities (when $\delta$ gets larger). The eigenvalues cross the real axis with non-zero imaginary part when the imposed flow velocity $v$ is lowered below a threshold. This bifurcation is shown in Fig. 8. The fastest growing eigenvector has the shape of a peak centered close to the fixed border, the distance between the peak and the border increasing with increasing imposed flow velocity.

To study this instability, we performed numerical simulations with Dirichlet boundary condition upstream and small imposed flow velocities. We observed that the system initially reaches a state similar to the one showed in Fig. 7, that is, a smooth connection between the boundary and the steady state. However, this solution becomes unstable

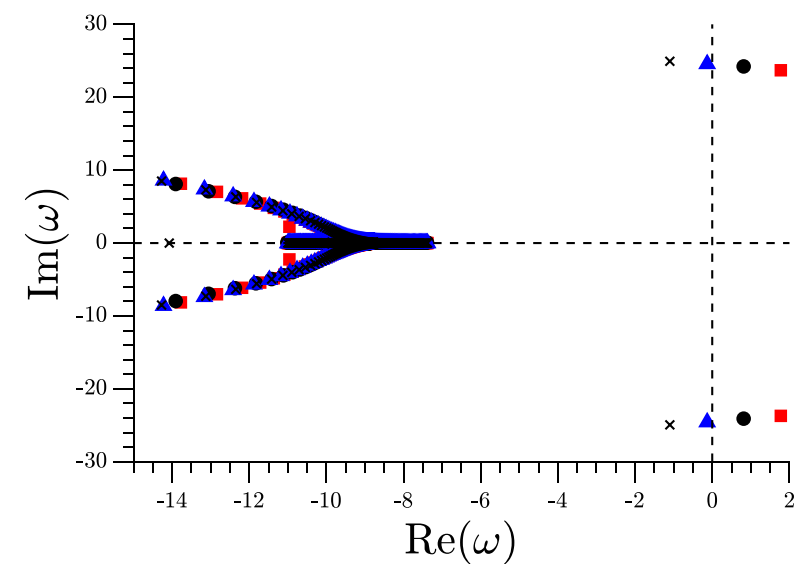

FIG. 8. Frequencies of the linear analysis of the monotone profile $\gamma=$ $\varphi_{0}+\delta \varphi_{1}$ showing the oscillatory bifurcation when the imposed flow $v$ is lowered. $v=1.32 \mathrm{~mm} / \mathrm{min}$ in red squares, $v=1.33 \mathrm{~mm} / \mathrm{min}$ in black circles, $v=1.34 \mathrm{~mm} / \mathrm{min}$ in blue triangles, and $v=1.35 \mathrm{~mm} / \mathrm{min}$ in black crosses. producing a peak which, as it grows, divides into two peaks. One of the peaks travels downstream and produces a wave train as was previously described in Sec. III. The second peak travels upstream until it reaches the boundary. Once the upstream travelling peak has been absorbed by the boundary, the system goes back to the smooth solution, which then again becomes unstable and repeats the cycle. This whole process generates periodically wave trains propagating downstream, as shown in Fig. 9.

The period of these perturbations is hard to measure downstream due to the wave train that it generates, whose period is given by the periodic travelling wave solution. To solve this, we measured the period of the initial destabilization peak at its point of creation, as shown in white in Fig. 9. This nucleation location moves farther away from the boundary as the imposed flow velocity increases. This relation is shown in Fig. 10.

This period $T$ does not appear to have a relation to any of the periods in the train wave previously studied. This, combined with the difference in the back and front velocities $v_{b}$ and $v_{f}$, produces phase slips. The phase slips occur when the front of the newly generated wave train catches up with the back of the previous wave train, thus forming downstream

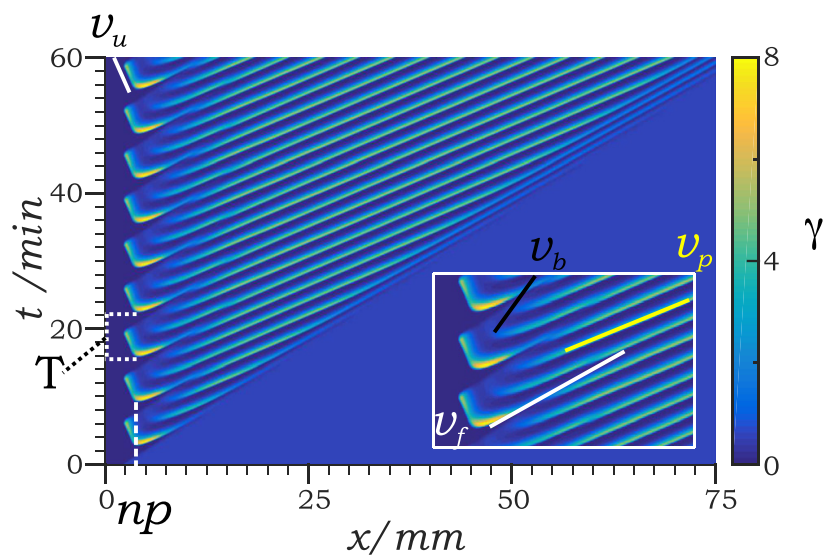

FIG. 9. Space-time plot of simulation performed in the convectively unstable regime using fixed (Dirichlet) boundary condition. Wave trains generated by the instability described in Sec. IV and measured quantities highlighted. The nucleation point is $n p$, where the destabilization occurs, $T$, the oscillations period, and $v_{u}$, the velocity of the upstream travelling peak. Inset with a zoom of the wave generation area with previously defined quantities of the wave train highlighted, $v_{b}$, velocity of the back of the wave train, $v_{f}$, velocity of the front of the wave train, and $v_{p}$, velocity of each individual peak. The imposed flow velocity is $1.2 \mathrm{~mm} / \mathrm{min}$. 

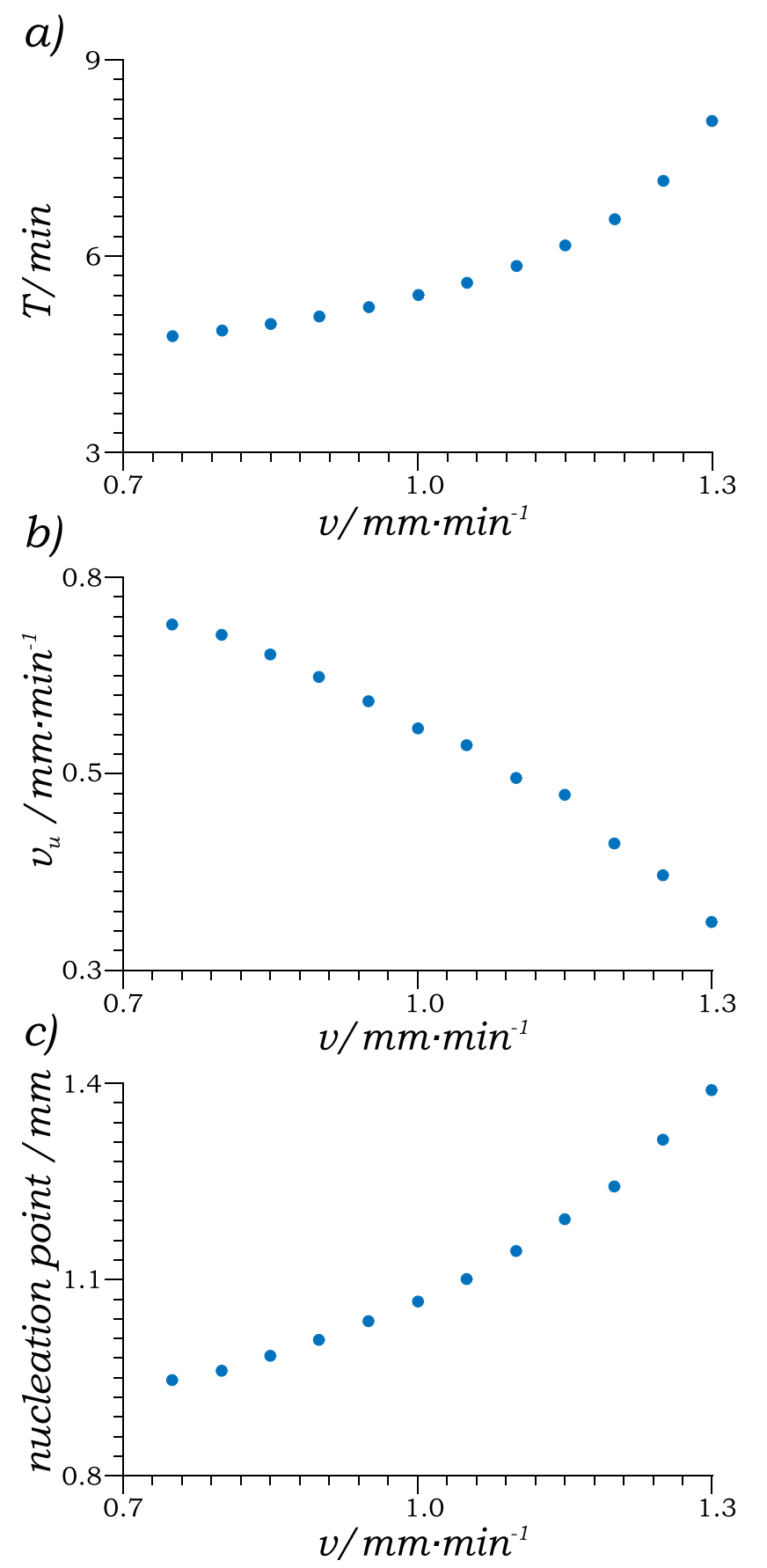

FIG. 10. Different properties of the boundary driven oscillations as function of the imposed flow velocity $v$. (a) Period of the oscillations, as measured at the point of nucleation of the instability. (b) Velocity of the upstream travelling peak. (c) Destabilization point position.

one larger wave train with phase slips. This process is highlighted on the inset of Fig. 9.

As expected, the velocity of the upstream travelling peak $v_{u}$ decreases with imposed flow velocity. Since the new wave does not appear until the previous one has travelled up to the boundary, the instability period is directly affected by the velocity of the upstream peak. Therefore, the period increases with increasing imposed flow velocities. All these dependencies are shown in Fig. 10.
The periodical travelling wave solutions selected by the boundary condition could not be measured for every velocity value, because of the interaction between the new wave train and the old one. This interaction produces numerous phase slips that change the wavelength along the wave train. For those values of $v$ where it was measured, the selected travelling wave falls into the stable range shown in Fig. 6.

We understand the upstream travelling peak as a trigger wave, analogous to the ones present in the excitable regime in this system. Trigger waves are non-linear excitation waves that propagate in excitable media when a perturbation above a threshold is applied. In these systems, small perturbations damp out but supra-threshold ones are amplified and excite the neighboring area allowing for wave propagation. ${ }^{45} \mathrm{~A}$ trigger wave has a velocity which is nonlinearly selected by the system. Another important characteristic is that a new trigger wave cannot enter the system until some recovery time has elapsed. In the case of the upstream travelling wave, the system cannot sustain another upstream travelling peak until the old one has reached the boundary and the cAMP close to the boundary has been washed away. Schematically, the wave works as follows. The cells closer to the boundary have been exposed to very small amounts of cAMP because it is initially washed away due to the boundary. As a result, they have a very high percentage of active receptors on the cell membrane. Therefore, they quickly react to the small perturbation of cAMP produced by the growing peak, emitting cAMP themselves and producing a trigger wave. It has been shown that trigger waves can travel against imposed flows when the advection is not too strong, experimentally in the Belousov-Zhabotinsky reaction ${ }^{46}$ and numerically in the excitable regime of the Martiel-Goldbeter model $^{47}$ and in the FitzHugh-Nagumo model. ${ }^{48}$

\section{2-DIMENSIONAL RESULTS}

To study the instability already investigated in one dimension, we performed numerical simulations in a 2 Dimensional system. The dimensions were chosen following the $D$. discoideum experiments of Gholami et al. ${ }^{31}$ In this microfluidic setup, the amoebas were placed in a $30 \mathrm{~mm}$ $\times 2 \mathrm{~mm} \times 100 \mu \mathrm{m}$ channel, where a constant flow was applied along the longest axis. Because of the small height and velocities of this system, the flow can be assumed to be laminar and constant in the long channel axis ( $x$-axis), thus making a Poiseuille flow. We solved the Navier-Stokes equation under these assumptions and used this flow as our imposed advection for the simulations. The resulting flow is parabolic in the short axis ( $z$-axis). This is the direction over which we averaged to have a 2-Dimensional system. In the $x y$-plane, the flow is almost planar in the center with a sharp boundary layer of the order of $50 \mu \mathrm{m}$ on the top $(y=2 \mathrm{~mm})$ and on the bottom $(y=0 \mathrm{~mm})$ boundaries, where the velocity quickly drops to zero. The detailed flow profile calculation is presented in the Appendix.

We performed numerical simulations with no-flux boundary conditions on the top and bottom boundaries and Dirichlet $[\rho, \gamma(x=0)=0]$ boundary condition upstream. The simulations confirmed our previous observations in one 


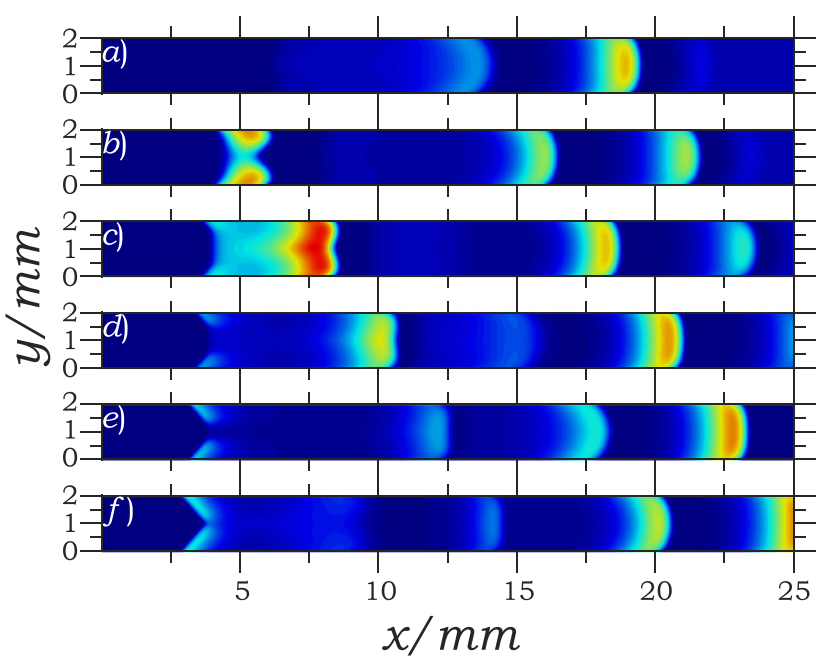

FIG. 11. Colormap of the concentration $\gamma$ every 0.9 min starting at $t=10.5 \mathrm{~min}$ at the top and increasing towards the bottom. Applied flow is $v=1.75 \mathrm{~mm} / \mathrm{min}$.

dimension: When a small advection flow is applied, an instability appears, which creates a wave train downstream and a travelling peak upstream. This process can be observed in Fig. 11, the destabilization peak begins to appear in Fig. 11(b), creating a train wave. The back travelling wave is already visible in Fig. 11(d) and more clear in Fig. 11(f).

Remarkable in comparison with the 1-Dimensional simulations are the range of existence of the instability and the shape of the upstream travelling peak. In the 2-Dimensional simulations, we observed that the system becomes stable at a higher speed $v=1.75 \mathrm{~mm} / \mathrm{min}$ compared to the 1Dimensional ones $v=1.33 \mathrm{~mm} / \mathrm{min}$, when measured at the center of the channel $\left(\sigma=0.45\right.$ and $\left.k_{e}=3.0\right)$. We attribute this difference to the smaller advection speeds at the boundary layer which are enough to destabilize the whole system. This phenomenon was also observed in some preliminary simulations using a parabolic advection flow, ${ }^{21}$ where the advection flow velocity is much smaller in a wider region, thus making the instability range of existence much larger.

Of particular interest is the shape that the upstream travelling peak acquires while it travels towards the boundary. Since this peak travels against the flow, its shape gets deformed due to the different speeds along the perpendicular axis. When the peak originally appears, it has a much flatter shape, similar to the imposed flow, as can be observed at the far right of Fig. 12. As the peak travels upstream (towards the left), it gets increasingly deformed until it acquires a

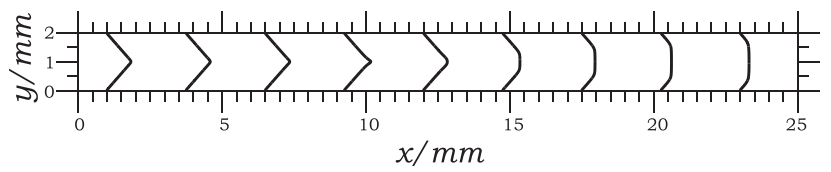

FIG. 12. Shape of the upstream travelling peak at different times taken as a contour at $\gamma=0.4$ and $v=1.75 \mathrm{~mm} / \mathrm{min}$. Lines taken every $0.5 \mathrm{~min}$, starting at $t=12 \mathrm{~min}$ on the right until $t=16 \mathrm{~min}$ on the left. Contours not in their original positions but spatially separated for better visualization.

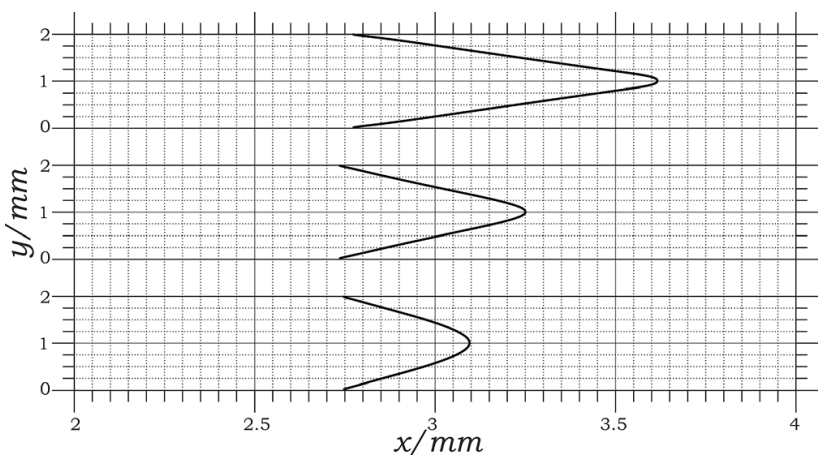

FIG. 13. Shape of the upstream travelling peak for different velocities taken as a contour at $\gamma=1.0$. Top: $v=1.75 \mathrm{~mm} / \mathrm{min}$, middle: $v=1.00 \mathrm{~mm} / \mathrm{min}$, bottom: $v=0.80 \mathrm{~mm} / \mathrm{min}$. Different scales are used in the $x$ - and $y$-axis for better visualisation.

triangular shape. Contours of the peak taken every $0.5 \mathrm{~min}$ are displayed in Fig. 12 showing this process.

The triangular deformation of a front due to an adverse flow was theoretically predicted by Edwards ${ }^{1}$ and experimentally confirmed by Leconte et al. ${ }^{2}$ for an auto-catalytic reaction. The main difference with our system is that in our reaction-diffusion-advection system, only the activator $\gamma$ is advected, while the inhibitor $\rho$ remains static. Like in those systems, the deformation of the wave is larger at larger imposed flows. This is shown in Fig. 13 for three different advection velocities. This wave deformation makes the characterization of the system difficult, because it produces different arrival times at the boundary. More work is needed in this direction to fully characterize this system in 2-D.

\section{CONCLUSIONS}

\section{A. No-flux boundary}

We have analyzed and characterized the convectively unstable regime in the model proposed by Martiel and Goldbeter for cAMP production in D. discoideum. In this regime, an initial perturbation generates a wave train of growing size (i.e., it contains more peaks as time passes) that travels downstream. In particular, the speed of the peaks located near the wave front (back) is higher (lower) than the advection flow, thus causing the growing size. These two velocities were numerically characterized through linear stability analysis and have an excellent agreement with the velocities measured in the nonlinear simulations of the model. The growing mode on the center of the wave train corresponds to one of the periodic travelling wave solutions of the system and moves faster than the front of the train. Therefore, a peak will move towards the front of the train, where then it will decrease its speed to match the front velocity. As a result of this smaller speed, the wavelength near the front of the train is smaller than in the center of the wave train, thus producing a traffic jam. These wave trains are similar to the differential flow induced convective instability (DIFICI) waves which were first predicted in Ref. 9, experimentally observed in Ref. 13 and further investigated in Refs. 14-16. 


\section{B. Fixed boundary}

When slow advection speeds are applied along with a Dirichlet (absorbing) boundary condition, an instability appears that periodically produces wave trains. This instability initially generates a peak that divides into two, with one peak travelling upstream towards the boundary and the other one producing a wave train downstream. Once the peak travelling upstream has reached the absorbing boundary, the process starts again, thus acting as a continuous source of waves. The velocity of the wave travelling upstream is affected by the imposed flow velocity. As expected, it travels slower at higher advection, and since the instability does not appear until the peak reaches the boundary, this affects the period of the oscillation. The faster the imposed flow, the longer the period. The location of appearance of this instability also increases with the advected flow velocity. We emphasize that these upstream travelling waves are nonlinearly selected and depend solely on the system parameters.

This instability was also observed in 2-Dimensional simulations, where the upstream travelling peak acquires the triangular shape of fronts propagating against adverse flows. ${ }^{1}$ This triangular shape increases its height with increasing advection flow. The instability persists up until higher velocities than in one dimension and similarly increases period with increased imposed flow.

The observed phenomena is different from other wave trains emitted by Dirichlet boundary conditions ${ }^{22}$ in that the waves are not directly emitted or absorbed by the boundary, but instead appear as a pair of waves from a nucleation point which exists downstream from the boundary. From this pair of waves, one travels upstream and is absorbed by the boundary while the other creates a wave train downstream. We expect that a similar mechanism may exist in systems where the convective or absolute unstable regime exists close to an excitable regime, thus facilitating the creation of an upstream travelling peak. This mechanism can then be used to produce a constant wave influx.

\section{ACKNOWLEDGMENTS}

The authors acknowledge A. Bae for fruitful discussions and unknown referees for insightful comments. E.V.H. thanks the Deutsche Akademische Austauschdienst (DAAD), Research Grants-Doctoral Programs in Germany. A.G. acknowledges MaxSynBio Consortium, which is jointly funded by the Federal Ministry of Education and Research of Germany and the Max Planck Society.

\section{APPENDIX: POSEUILLE FLOW CALCULATION}

To estimate the flow profile inside the channel, we used the Navier-Stokes equations and assumed incompressible flow in a $3 \mathrm{D}$ rectangular geometry $(x \in[0, L], y \in[-c, c]$, $z \in[-b, b])$, with zero velocity as the boundary condition along the two shortest directions, $\boldsymbol{u}(y= \pm c)=0$ and $\boldsymbol{u}(z=$ $\pm b)=0$, thus obtaining

$$
\rho \frac{D \boldsymbol{u}}{D t}=\rho \boldsymbol{g}-\nabla p+\mu \nabla^{2} \boldsymbol{u}
$$

where bold text denotes vectors, $\mu$ is the system viscosity, $\rho$ its density, $p$ the pressure, and $\boldsymbol{u}$ the fluid velocity. We further simplified by assuming that the flow is constant over time, only exists in the $\hat{x}$-direction, and is constant over this direction, that is, $\boldsymbol{u}=u \hat{x}$ and $\partial_{x} u=\partial_{t} u=0$; therefore, the previous equation reduces to

$$
\mu\left(\frac{\partial^{2} u}{\partial y^{2}}+\frac{\partial^{2} u}{\partial z^{2}}\right)=\frac{\partial p}{\partial x} \equiv-G
$$

where $G$ is an externally applied pressure difference. This can be solved by setting the auxiliary function

$$
\mathcal{F}=u-\frac{G\left(b^{2}-z^{2}\right)}{2 \mu},
$$

which reduces the system to solve $\nabla^{2} \mathcal{F}=0$ with boundary conditions $\mathcal{F}(z= \pm b)=0$ and $\mathcal{F}(y= \pm c)=-G\left(b^{2}-z^{2}\right) /$ $2 \mu$. Using variable separation $\mathcal{F}(y, z)=F_{y}(y) F_{z}(z)$ and considering the symmetry of the system, we obtain $F_{z}=\cos \left(k_{z} z\right)$, $F_{y}=\cosh \left(k_{y} y\right)$ with $k_{z}=k_{y}$. The boundary condition $F_{z}(z=$ $\pm b)=0$ sets

$$
k_{z}=k_{y}=\frac{m \pi}{2 b}
$$

with $m$ an odd integer. The other boundary condition is fulfilled by using Fourier series, finally obtaining

$$
u=\frac{G\left(b^{2}-z^{2}\right)}{2 \mu}+\sum_{\text {odd }} A_{n} \cosh \left(\frac{n \pi}{2 b} y\right) \cos \left(\frac{n \pi}{2 b} z\right),
$$

where

$$
A_{n}=-\frac{16 G b^{2}}{\mu \pi^{3} n^{3}} \frac{\sin \left(\frac{n \pi}{2}\right)}{\cosh \left(\frac{n \pi c}{2 b}\right)}
$$

with $c=1 \mathrm{~mm}$ and $b=50 \mu \mathrm{m}$. Since in our case the $z$ direction is much shorter than the others, this is the length that sets the boundary layer in the system. Therefore, the flow looks parabolic in the $z$-axis, but almost planar in the $y$-axis, with a very sharp drop to zero close to the boundaries.

${ }^{1}$ B. F. Edwards, "Poiseuille advection of chemical reaction fronts," Phys. Rev. Lett. 89, 104501 (2002).

${ }^{2}$ M. Leconte, J. Martin, N. Rakotomalala, and D. Salin, "Pattern of reaction diffusion fronts in laminar flows," Phys. Rev. Lett. 90, 128302 (2003).

${ }^{3}$ A. Zaikin and A. Zhabotinsky, "Concentration wave propagation in twodimensional liquid-phase self-oscillating system," Nature 225, 535-537 (1970).

${ }^{4}$ A. T. Winfree, "Spiral waves of chemical activity," Science 175, 634-636 (1972).

${ }^{5}$ M. A. Allessie, F. I. Bonke, and F. J. Schopman, "Circus movement in rabbit atrial muscle as a mechanism of tachycardia," Circ. Res. 33, 54-62 (1973).

${ }^{6} \mathrm{~S}$. Kondo and R. Asai, "A reaction-diffusion wave on the skin of the marine angelfish pomacanthus," Nature 376, 765 (1995).

${ }^{7}$ J. Lechleiter, S. Girard, E. Peralta, and D. Clapham, "Spiral calcium wave propagation and annihilation in xenopus laevis oocytes," Science 252, 123-126 (1991).

${ }^{8}$ P. Devreotes, "Dictyostelium discoideum: A model system for cell-cell interactions in development," Science 245, 1054-1058 (1989). 
${ }^{9}$ A. B. Rovinsky and M. Menzinger, "Chemical instability induced by a differential flow," Phys. Rev. Lett. 69, 1193 (1992).

${ }^{10}$ R. J. Deissler, "Noise-sustained structure, intermittency, and the Ginzburg-Landau equation,” J. Stat. Phys. 40, 371-395 (1985).

${ }^{11}$ B. Sandstede and A. Scheel, "Absolute and convective instabilities of waves on unbounded and large bounded domains," Phys. D: Nonlinear Phenom. 145, 233-277 (2000).

${ }^{12}$ R. Satnoianu, J. Merkin, and S. Scott, "Interaction between hopf and convective instabilities in a flow reactor with cubic autocatalator kinetics," Phys. Rev. E 57, 3246 (1998).

${ }^{13}$ A. B. Rovinsky and M. Menzinger, "Self-organization induced by the differential flow of activator and inhibitor," Phys. Rev. Lett. 70, 778 (1993).

${ }^{14}$ R. Toth, A. Papp, V. Gaspar, J. Merkin, S. Scott, and A. Taylor, "Flowdriven instabilities in the Belousov-Zhabotinsky reaction: Modelling and experiments," Phys. Chem. Chem. Phys. 3, 957-964 (2001).

${ }^{15}$ R. A. Satnoianu, J. H. Merkin, and S. K. Scott, "Spatio-temporal structures in a differential flow reactor with cubic autocatalator kinetics," Phys. D: Nonlinear Phenom. 124, 345-367 (1998).

${ }^{16}$ R. A. Satnoianu, J. H. Merkin, and S. K. Scott, "Pattern formation in a differential-flow reactor model," Chem. Eng. Sci. 55, 461-469 (2000).

${ }^{17} \mathrm{P}$. S. Hagan, "Spiral waves in reaction-diffusion equations," SIAM J. Appl. Math. 42, 762-786 (1982).

${ }^{18}$ I. S. Aranson and L. Kramer, "The world of the complex GinzburgLandau equation,” Rev. Mod. Phys. 74, 99 (2002).

${ }^{19}$ L. M. Pismen, Vortices in Nonlinear Fields: From Liquid Crystals to Superfluids, from Non-Equilibrium Patterns to Cosmic Strings (Oxford University Press, 1999), Vol. 100.

${ }^{20}$ A. Gholami, O. Steinbock, V. Zykov, and E. Bodenschatz, "Flow-driven instabilities during pattern formation of Dictyostelium discoideum," New J. Phys. 17, 063007 (2015).

${ }^{21}$ A. Gholami, V. Zykov, O. Steinbock, and E. Bodenschatz, "Flow-driven two-dimensional waves in colonies of Dictyostelium discoideum," New J. Phys. 17, 093040 (2015).

${ }^{22}$ J. A. Sherratt, "Periodic travelling wave selection by dirichlet boundary conditions in oscillatory reaction-diffusion systems," SIAM J. Appl. Math. 63, 1520-1538 (2003)

${ }^{23}$ J.-L. Martiel and A. Goldbeter, "A model based on receptor desensitization for cyclic AMP signaling in Dictyostelium cells," Biophys. J. 52, 807 (1987).

${ }^{24}$ R. Merson, "An operational method for the study of integration processes," in Proceedings of the Symposium on Data Processing (1957), pp. $1-25$.

${ }^{25} \mathrm{~J}$. Bonner, "Evidence for the formation of aggregates by chemotaxis in the development of the slime mold Dictyostelium discoideum," J. Exp. Zool. 106, 1 (1947).

${ }^{26}$ W. B. Loomis, The Development of Dictyostelium Discoideum (Elsevier, 1982).

${ }^{27}$ C. Parent and P. Devreotes, "Molecular genetics of signal transduction in Dictyostelium," Annu. Rev. Biochem. 65, 411-440 (1996).

${ }^{28}$ M. Dinauer, T. Steck, and P. Devreotes, "Cyclic, $3^{\prime}, 5^{\prime}$-AMP relay in Dictyostelium discoideum. V. Adaptation of the cAMP signaling response during cAMP stimulation," J. Cell Biol. 86, 554 (1980).
${ }^{29}$ B. M. Shaffer, "Secretion of cyclic AMP induced by cyclic AMP in the cellular slime mould Dictyostelium discoideum," Nature 255, 549-552 (1975).

${ }^{30}$ J. J. Tyson, K. A. Alexander, V. Manoranjan, and J. Murray, "Spiral waves of cyclic AMP in a model of slime mold aggregation," Phys. D: Nonlinear Phenom. 34, 193-207 (1989).

${ }^{31}$ A. Gholami, O. Steinbock, V. Zykov, and E. Bodenschatz, "Flow-driven waves and phase-locked self-organization in quasi-one-dimensional colonies of Dictyostelium discoideum," Phys. Rev. Lett. 114, 018103 (2015).

${ }^{32}$ J. Lauzeral, J. Halloy, and A. Goldbeter, "Desynchronization of cells on the developmental path triggers the formation of spiral waves of cAMP during Dictyostelium aggregation," Proc. Natl. Acad. Sci. 94, 9153-9158 (1997).

${ }^{33}$ L. Brevdo, "A study of absolute and convective instabilities with an application to the eady model," Geophys. Astrophys. Fluid Dyn. 40, 1-92 (1988).

${ }^{34}$ L. Brevdo, P. Laure, F. Dias, and T. J. Bridges, "Linear pulse structure and signalling in a film flow on an inclined plane," J. Fluid Mech. 396, 37-71 (1999).

${ }^{35}$ R. J. Briggs, Electron-Stream Interaction with Plasmas (MIT, 1964).

${ }^{36}$ L. Brevdo, "Convectively unstable wave packets in the blasius boundary layer," Z. Angew. Math. Mech. 75, 423-436 (1995).

${ }^{37}$ W. Van Saarloos, "Front propagation into unstable states," Phys. Rep. 386, 29-222 (2003).

${ }^{38} \mathrm{M}$. Holzer and A. Scheel, "Criteria for pointwise growth and their role in invasion processes," J. Nonlinear Sci. 24, 661-709 (2014).

${ }^{39}$ N. Kopell and L. Howard, "Plane wave solutions to reaction-diffusion equations," Stud. Appl. Math. 52, 291-328 (1973).

${ }^{40}$ J. A. Sherratt, "Numerical continuation methods for studying periodic travelling wave (wavetrain) solutions of partial differential equations," Appl. Math. Comput. 218, 4684-4694 (2012).

${ }^{41}$ J. D. Rademacher, B. Sandstede, and A. Scheel, "Computing absolute and essential spectra using continuation," Phys. D: Nonlinear Phenom. 229, 166-183 (2007).

${ }^{42}$ J. A. Sherratt, "Numerical continuation of boundaries in parameter space between stable and unstable periodic travelling wave (wavetrain) solutions of partial differential equations," Adv. Comput. Math. 39, 175-192 (2013).

${ }^{43}$ C. T. Hamik and O. Steinbock, "Shock structures and bunching fronts in excitable reaction-diffusion systems," Phys. Rev. E 65, 046224 (2002).

${ }^{44}$ N. Manz and O. Steinbock, "Dynamics of excitation pulses with attractive interaction: Kinematic analysis and chemical wave experiments," Phys. Rev. E 70, 066213 (2004).

${ }^{45}$ V. S. Zykov and A. T. Winfree, Simulation of Wave Processes in Excitable Media (John Wiley \& Sons, Inc., 1992)

${ }^{46}$ K. Agladze, M. Braune, H. Engel, H. Linde, and V. Krinsky, “Autowave propagation in a Belousov-Zhabotinsky medium with immobilized catalyst and stationary flow of reagents," Z. Phys. Chem. 173, 79-85 (1991).

${ }^{47}$ J. Lindner, H. Ševčíková, and M. Marek, "Influence of an external electric field on cAMP wave patterns in aggregating Dictyostelium discoideum," Phys. Rev. E 63, 041904 (2001).

${ }^{48}$ E. A. Ermakova, E. E. Shnol, M. A. Panteleev, A. A. Butylin, V. Volpert, and F. I. Ataullakhanov, "On propagation of excitation waves in moving media: The FitzHugh-Nagumo model,” PloS One 4, e4454 (2009). 


\section{$3 \quad$ Influence of Fast Advective Flows on Pattern Formation in Dictyostelium discoideum}

The following paper originally appeared as Eckstein, T., Vidal-Henriquez, E., Bae, A., Zykov, V., Bodenschatz, E., \& Gholami, A. (2018). Influence of fast advective flows on pattern formation of Dictyostelium discoideum. PloS one, 13(3), e0194859.

doi: 10.1371/journal.pone.0194859. It is licensed under CC BY 4.0.

EVH performed numerical simulations and analysis. EVH, TE, and AG designed and planned research, EVH, TE, and AG wrote the manuscript. All authors reviewed and edited the manuscript. Figures 1 b-c), 6, 7, 8, 9, 12, and 14 by EVH. From the electronically available supplementary material S1 File, S1 Fig, S6 Video, S7 Video, S8 Video, S9 Video, S11 Video, and S13 Video are by EVH. 


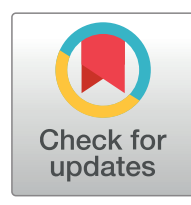

\section{G openaccess}

Citation: Eckstein T, Vidal-Henriquez E, Bae A, Zykov V, Bodenschatz E, Gholami A (2018) Influence of fast advective flows on pattern formation of Dictyostelium discoideum. PLOS ONE 13(3): e0194859. https://doi.org/10.1371/journal. pone. 0194859

Editor: Jun Ma, Lanzhou University of Technology, CHINA

Received: November 30, 2017

Accepted: March 12, 2018

Published: March 28, 2018

Copyright: ๑ 2018 Eckstein et al. This is an open access article distributed under the terms of the Creative Commons Attribution License, which permits unrestricted use, distribution, and reproduction in any medium, provided the original author and source are credited.

Data Availability Statement: All relevant data are within the paper and its Supporting Information files.

Funding: T. E. acknowledges Deutsche Forschungsgemeinschaft (DFG), project number GH 184/1-1. E.V.H. thanks the Deutsche Akademische Austauschdienst (DAAD), Research Grants-Doctoral Programs in Germany. A.G. acknowledges MaxSynBio Consortium, which is jointly funded by the Federal Ministry of Education and Research of Germany and the Max Planck

\section{RESEARCH ARTICLE \\ Influence of fast advective flows on pattern formation of Dictyostelium discoideum}

\author{
Torsten Eckstein $^{1 \odot}$, Estefania Vidal-Henriquez ${ }^{1 \odot}$, Albert Bae ${ }^{1}$, Vladimir Zykov ${ }^{1}$, \\ Eberhard Bodenschatz ${ }^{1,2,3}$, Azam Gholami ${ }^{1 *}$
}

1 Max Planck Institute for Dynamics and Self-Organization, 37077, Göttingen, Germany, 2 Institute for Nonlinear Dynamics, University of Göttingen, 37073, Göttingen, Germany, 3 Laboratory of Atomic and SolidState Physics and Sibley School of Mechanical and Aerospace Engineering, Cornell University, Ithaca, New York 14853, United States of America

- These authors contributed equally to this work.

*azam.gholami@ds.mpg.de

\begin{abstract}
We report experimental and numerical results on pattern formation of self-organizing Dictyostelium discoideum cells in a microfluidic setup under a constant buffer flow. The external flow advects the signaling molecule cyclic adenosine monophosphate (cAMP) downstream, while the chemotactic cells attached to the solid substrate are not transported with the flow. At high flow velocities, elongated CAMP waves are formed that cover the whole length of the channel and propagate both parallel and perpendicular to the flow direction. While the wave period and transverse propagation velocity are constant, parallel wave velocity and the wave width increase linearly with the imposed flow. We also observe that the acquired wave shape is highly dependent on the wave generation site and the strength of the imposed flow. We compared the wave shape and velocity with numerical simulations performed using a reaction-diffusion model and found excellent agreement. These results are expected to play an important role in understanding the process of pattern formation and aggregation of $D$. discoideum that may experience fluid flows in its natural habitat.
\end{abstract}

\section{Introduction}

In a reaction-diffusion-advection system one or more reacting species are advected downstream with an externally imposed velocity. This advective flow can induce unique emergent phenomena. An eminent example is the differential flow induced chemical instability (DIFICI) that destabilizes an otherwise spatially homogeneous state of a system [1-3]. The basic idea behind this is that the reacting species flow at different rates. This differential transport can initiate instabilities in an otherwise spatially homogeneous state of the system, leading to propagating wave packets of reactant concentrations traveling in the flow direction. This mechanism of generating spatial structures is free from the constrains of the Turing mechanism [4], which requires a large difference in diffusion coefficients of the two species involved. Accordingly one can expect DIFICI to be found widely in population dynamics [5-8] and biological morphogenesis [9]. 
Society. The funders had no role in study design, data collection and analysis, decision to publish, or preparation of the manuscript.

Competing interests: The authors have declared that no competing interests exist.
The aggregation of $D$. discoideum amoeba after nutrient deprivation is one of the best model systems for the study of spatial-temporal pattern formation at the multicellular level. Upon starvation, $D$. discoideum starts a developmental program as a surviving mechanism. The first part of this process consists of aggregation of $10^{4}-10^{5}$ chemotactic cells to form a migrating slug, which then act as a multicellular organism to search for nutrients. Because of this, $D$. discoideum has been largely studied to understand the transition from uni- towards multicellularity. The aggregation of amoebas is achieved by using the signaling chemical cyclic adenosine monophosphate (cAMP), which is initially secreted by some of the amoebas and then relayed by the others. The patterns produced by cAMP have attracted a lot of attention in the pattern formation community, since they are a primary example of spiral waves and target patterns in nature. Regarding spiral waves and target patterns in D. discoideum see for example Refs. [10-13]. These structures then constitute the centers to which the amoebas aggregate. However, in their natural habitat in the forest soil, $D$. discoideum cells are subjected to flows which advect cAMP, thus affecting the signaling process. In soils, rainwater speeds can vary from values near zero up to around $250 \mathrm{~mm} / \mathrm{min}$, which is one order of magnitude larger than flow rates studied in this work [14]. It is not yet clear how these advective flows affect the aggregation of $D$. discoideum cells in nature.

Recently, we have conducted experiments and performed numerical simulations to study flow-driven waves in a biological system, namely quasi one-dimensional colonies of signaling amoeba $D$. discoideum [15]. In these experiments with chemotactically competent $D$. discoideum cells, a straight flow-through microfluidic channel was used. Starved cells were allowed to settle on the substrate before a laminar flow of buffer was switched on. The flow advected extracellular cAMP downstream but was not strong enough to detach the cells from the substrate. This differential transport of extracellular cAMP induced macroscopic wave trains that had a unique period and propagated with a velocity proportional to the imposed flow velocity downstream. This behavior was studied theoretically $[16,17]$ using the two-component reaction-diffusion model proposed by Martiel-Goldbeter [18] for the production and relay of cAMP. While the theoretical results could explain much of the experimental observations, there were still open questions regarding the generation of a self supporting wave train at the inlet of the microfluidic channel and only small flow rates of up to $5 \mathrm{~mm} / \mathrm{min}$ were studied. Furthermore, the state of the cells was assumed to be constant in the convectively unstable regime, lacking a way to verify this experimentally.

In this work, we extend our experiments to investigate flow-driven waves at high flow rates in the same microfluidic set up. The flows are not yet strong enough to detach the cells from the substrate. We observed elongated waves that extend over the whole length of the channel and propagate both perpendicular and parallel to the flow direction. We characterized the wave shape as well as the wave propagation velocity and compared them to the numerical simulations of the system. Moreover, we changed the imposed flow rates abruptly to study the system response both experimentally and by means of numerical simulations. In our comprehensive numerical study, we found that the two-component Martiel-Goldbeter model does not correctly reproduce the wave shape observed experimentally at a higher flow velocities. However, a three-component approach successfully reproduces the wave shape while still matching with the experimental results in period and wave speed. Additionally, we found that sustained wave formation can be induced using a developmental path model [19] for the state of the cells in the channel. This seems a very reasonable assumption, since $D$. discoideum cells change the activity of a number of genes during the aggregation process [20]. We could also reproduce the experimental observations for rapid flow switching by assuming a mixture of oscillatory and excitable cells. 


\section{Materials and methods}

\section{Cell culture}

All experiments were performed with $D$. discoideum AX2-214 cells, kindly provided by Günther Gerisch (MPI for Bio-chemistry, Martinsried, Germany). Cells were grown in HL-5 medium (35.5 $\mathrm{g}$ of Formedium powder from Formedium Ltd, England, per liter of doubledistilled water, autoclaved and filtered) at $22^{\circ} \mathrm{C}$ on polystyrene Petri dishes (TC Dish 100, Sarsted, Germany) and harvested when they became confluent. Before the experiments, the cells were centrifuged and washed two times with phosphate buffer $\left(2 g\right.$ of $\mathrm{KH}_{2} \mathrm{PO}_{4}$ and $0.36 g$ of $\mathrm{Na}_{2} \mathrm{HPO}_{4} \cdot \mathrm{H}_{2} \mathrm{O}$ per liter at $\mathrm{pH}$ 6.0, autoclaved, both from Merck, Germany). The centrifuged cells were resuspended in $10 \mathrm{ml}$ of the same buffer and transferred into a shaking Erlenmeyer flask $(150 \mathrm{rpm})$ for starvation. After approximately one hour, the cells were centrifuged at $1000 \mathrm{rpm}$ for $3 \mathrm{~min}$ and resuspended in $200 \mu \mathrm{l}$ fresh phosphate buffer. The cell density was determined using a hemocytometer (Neubauer Zählkammer), diluted to $5 \times 10^{7}$ cells $/ \mathrm{ml}$ of phosphate buffer and filled into the microfluidic channel.

\section{Microfluidics}

The microfluidic devices were fabricated by standard soft lithography [21]. A silicon wafer was coated with a $100 \mu \mathrm{m}$ photoresist layer (SU-8 100, Micro Resist Technology GmbH, Berlin, Germany) and patterned by photolithography to obtain a structured master wafer. The channels are $2 \mathrm{~mm}$ wide, $50 \mathrm{~mm}$ long, and $103 \pm 2 \mu \mathrm{m}$ high. Polydimethylsiloxane (PDMS, 10:1 mixture with curing agent, Sylgard 184, Dow Corning GmbH, Wiesbaden, Germany) was poured onto the wafer and cured for $2 \mathrm{~h}$ at $75^{\circ} \mathrm{C}$. To produce the microfluidic device, a PDMS block containing the macro-channels was cut out, and two inlets $(7 \mathrm{~mm}$ and $0.75 \mathrm{~mm}$ in diameter) were punched through the PDMS at opposite ends of the channel with the help of PDMS punchers (Harris Uni-Core-7.00 and Harris Uni-Core-0.75). Afterwards, a glass microscope slide $(76 \times 26 \mathrm{~mm}, \mathrm{VWR})$ was sealed to the PDMS block following a 20-30 s treatment in air plasma (PDC 002, Harrick Plasma, Ithaca, USA) to close the macro-channels. The large inlet was used as a liquid reservoir and from the other side phosphate buffer was pumped out using a high precision syringe pump (PHD 2000 Infuse/Withdraw Syringe Pump from Harvard Apparatus, USA, combined with gasstight glass syringes from Hamilton, USA) at constant buffer flow rate. Moreover, given the dimension of the channel and the dynamics viscosity of the flowing phosphate buffer $\left(\eta=10^{-3} \mathrm{~Pa} \mathrm{~s}\right)$, one can calculate the shear stress applied on the cells at the highest imposed flow velocity of $V_{f}=50 \mathrm{~mm} / \mathrm{min}$ to be $\sigma=0.046 \mathrm{~Pa}$ (see supplementary S1 File). According to the literature, mechanosensing in D. discoideum has been observed above a threshold of $\sigma=0.7 \mathrm{~Pa}$, and cell detachment from substrate occur at higher threshold of $\sigma=2.7 \mathrm{~Pa}$ [22]. We are thus one order of magnitude below the regime where flow induced shear stress would bias the motion of chemotactic cells or even detach the cells from substrate.

\section{Image acquisition and analysis}

We used a dark-field setup consisting of a monochrome 12-bit CCD camera (QIClick-F-M-12 from QImaging), a $50 \mathrm{~mm}$ focal length objective (MVL50M23 from Thor Labs), a 7 inch focal length fresnel lens (11.0" x 11.0", 7" Focal Length from Edmund Optics, bottom side in-house coated with an anti-reflective coating) and a ring of green LEDs as light source (LED Miniatur Ringbeleuchtung LSR24-G from LUMIMAX). The camera was controlled with an image capture program (Micro-Manager [23]) and recorded images every 20 seconds. To process darkfield images, we first subtract them from each other (image number $n$ from image number 
$n+3)$ [24] and then band-passed filtered where large structures are filtered down to $3.5 \mathrm{~mm}$ and small structures up to $0.294 \mathrm{~mm}$. Finally, to calculate the phase map, at each pixel we first subtracted the time average of the signal and then performed the Hilbert transform [25].

\section{Numerical simulations}

We conducted numerical simulations of the model proposed by Martiel and Goldbeter [18] for the production and relay of CAMP, with the addition of an advection term to account for the imposed flow (see Fig 1a). The reaction-diffusion set of equations model (in 2-D) the amount of cAMP in the extracellular medium $\gamma(x, y)$, the amount of cAMP in the intracellular medium $\beta(x, y)$, and the percentage of active receptors on the outside of the cell membrane $\rho(x, y)$, where $x, y$ are cartesian spatial coordinates. This last field $\rho(x, y)$ quantifies the affinity of the cell receptors to bind with CAMP, thus providing the refractory time for this excitable medium. We use a 2-D approximation of the shape of the channel ( $x-y$ plane in Fig $2 b)$ due to its low aspect ratio in $z$ direction. The equations are as follow

$$
\begin{gathered}
\partial_{t} \rho=k_{1}\left[-f_{1}(\gamma) \rho+f_{2}(\gamma)(1-\rho)\right], \\
\partial_{t} \beta=q \sigma \alpha \Phi(\rho, \gamma) /(1+\alpha)-\left(k_{i}+k_{t}\right) \beta, \\
\partial_{t} \gamma=D \nabla^{2} \gamma-v(y) \cdot \nabla \gamma+k_{t} \beta / h-k_{e} \gamma,
\end{gathered}
$$

with

$$
f_{1}(\gamma)=\frac{1+\kappa \gamma}{1+\gamma}, \quad f_{2}(\gamma)=\frac{\mathcal{L}_{1}+\kappa \mathcal{L}_{2} c \gamma}{1+c \gamma}, \quad \Phi(\rho, \gamma)=\frac{\lambda_{1}+Y^{2}}{\lambda_{2}+Y^{2}}, \quad Y(\gamma, \rho)=\frac{\rho \gamma}{1+\gamma},
$$

$\nabla=\partial_{x} \hat{x}+\partial_{y} \hat{y}$, and $v(y)=v(y) \hat{x}$. Eq la models the process of desensitization and recovery of the active receptors given by $f_{1}$ and $f_{2}$, respectively. Eq $1 \mathrm{~b}$ characterizes the changes in cAMP
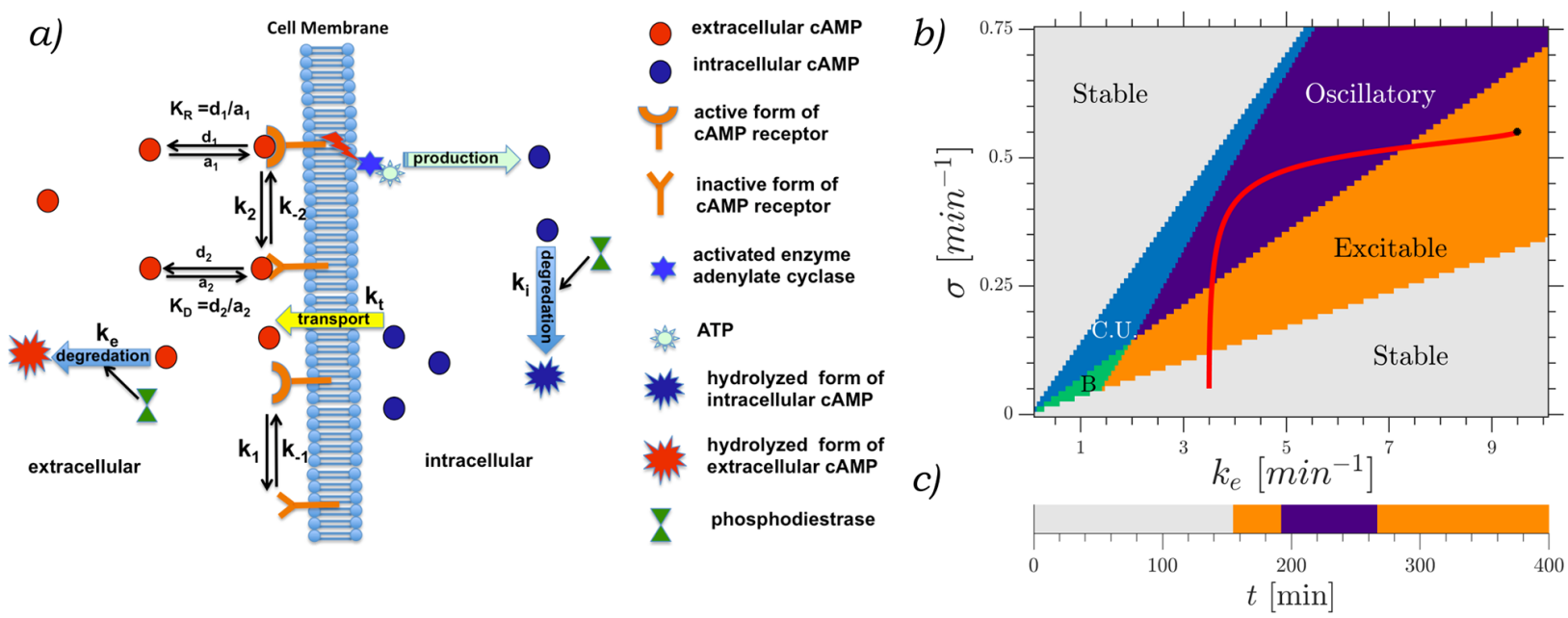

Fig 1. a) Schematic representation of the reaction-diffusion model used, reproduced from [16]. b) Phase diagram showing the different regimes depending on the production $\sigma$ and degradation $k_{e}$. Stable regime in white, where one stable steady state exists, excitable regime in orange, 3 steady states, one of which is excitable and the other two unstable. Oscillatory regime in purple, one unstable steady state surrounded by a limit cycle. Convectively unstable regime in light blue, one steady state which is convectively unstable. Bistable regime in green, two stable steady states. The red line marks the trajectory that the developmental path follows. Simulations with fixed parameters used the ones marked by the black asterisk. c) Cell state over time for a cell starting with $t_{s}=0$. The color coding is the same as b).

https://doi.org/10.1371/journal.pone.0194859.g001 


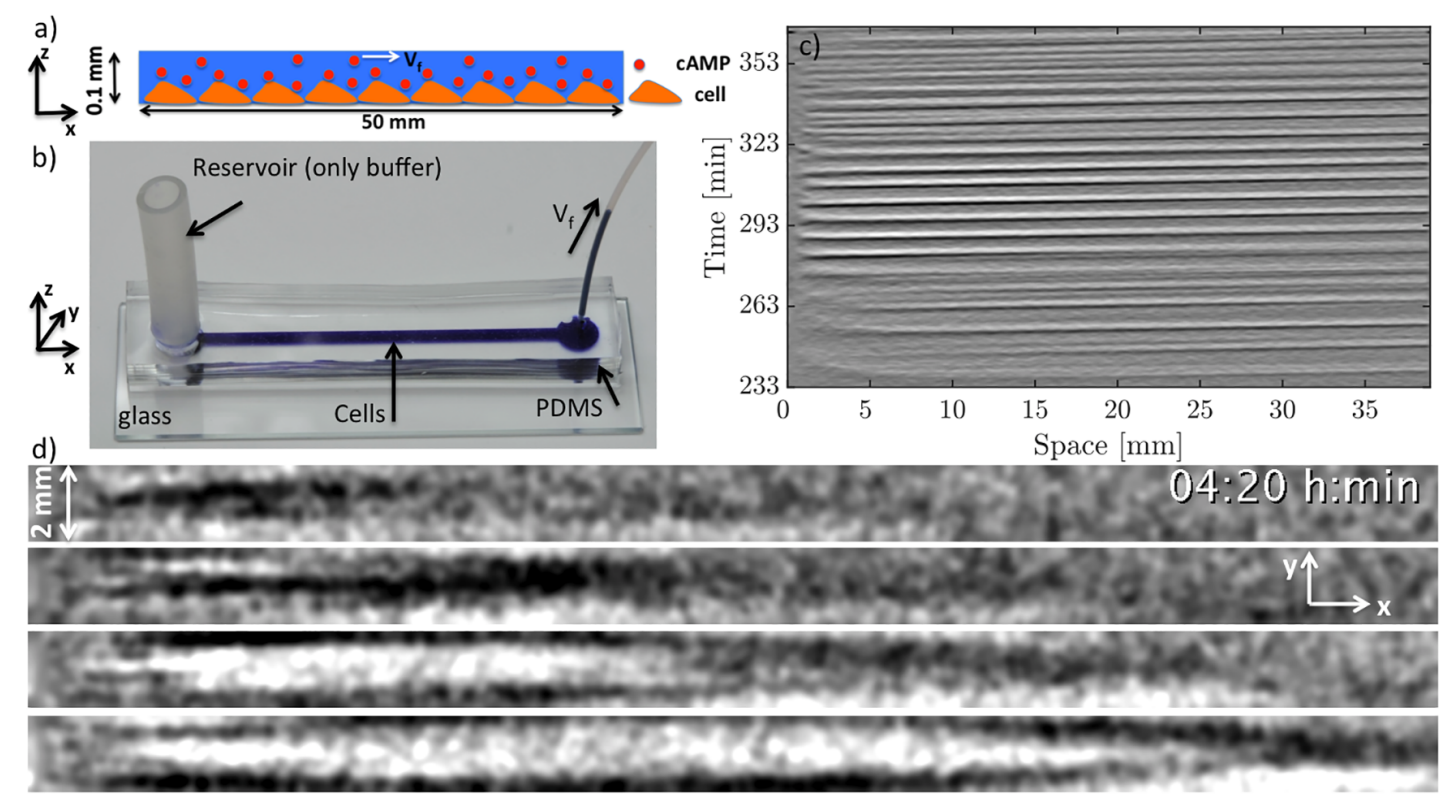

Fig 2. a) A schematic side view of the channel loaded with cells that are attached to the substrate and exposed to an external fluid flow advecting cAMP molecules downstream. b) Experimental setup filled with blue ink for better visualization. The reservoir is filled only with buffer and the liquid is pumped out with a syringe pump from the right side. c) Space-time plot of the flow-driven waves at the imposed flow velocity of $V_{f}=10 \mathrm{~mm} / \mathrm{min}$. d) Snapshots of the waves taken from the top of the channel obtained by subtracting successive images (captured every $20 \mathrm{sec}$ ) of the channel every 1 min (image number $n+3$ minus image number $n$ ) and bandpass filtered. The time increment between successive images is $1 \mathrm{~min}$. Time stamp shows the time since the start of starvation.

https://doi.org/10.1371/journal.pone.0194859.g002

inside the cells given by the nonlinear production term $\Phi$. The amount of intracellular cAMP is reduced by intracellular degradation and transport to the extracellular medium at rates described by $k_{i}$ and $k_{t}$, respectively. Finally, Eq $1 \mathrm{c}$ represents the changes on $\gamma$ given by degradation through phosphodiesterase at a rate $k_{e}$ and the transport from the intracellular medium at a rate $k_{t}$. These processes are schematically represented in Fig 1a. $\gamma$ is subjected to diffusion and advection, the other two fields do not diffuse nor advect since they are attached to the cells. For a detailed derivation of this model please refer to the original works of Martiel and Goldbeter [18] and Tyson et. al. [26]. The parameters used are $k_{1}=0.09 \mathrm{~min}^{-1}, \kappa=18.5$, $\mathcal{L}_{1}=10, \mathcal{L}_{2}=0.005, c=10, q=4000, \alpha=3, \lambda_{1}=10^{-4}, \lambda_{2}=0.2575, k_{i}=1.7 \mathrm{~min}^{-1}, k_{t}=0.9$ $\min ^{-1}, D=0.024 \mathrm{~mm}^{2} / \mathrm{min}, h=5$.

We simulated this system using a Runge-Kutta scheme with a Merson error aproximation [27] to ensure numerical accuracy. Nonlinear discretization was used for the advection operator in order to deal with high velocities while keeping a non-negative concentration of cAMP [28]. We used a no flux $\left(\partial_{x} \gamma(x=0)=0\right)$ boundary condition in all boundaries, including upstream, and kept the same parameters as in our previous simulations [29] while keeping freedom to move in the parameter space characterized by $\sigma$ and $k_{e}$. We conducted simulations both with fixed parameters (over time and space) and with a developmental path based on the work by Laurenzal et al [19]. When we used this path, the parameters $k_{e}$ and $\sigma$ were changed from being uniform in the whole system to being particular to each cell group (patch). Each patch had an area of $0.1 \mathrm{~mm} \times 0.1 \mathrm{~mm}$ and a particular starting time along the cellular developmental path. This path takes the cells from having one stable solution, to an excitable regime, one oscillatory solution, and then back to excitable (see Fig $1 \mathrm{~b}$ for an overview of the 
different regimes in this system), by changing with time the parameters $\sigma$ and $k_{e}$ according to

$$
\sigma(t)=0.3+0.25 \tanh \left(\frac{t+t_{s}-200}{50}\right), \quad k_{e}(t)=6.5+3 \tanh \left(\frac{t+t_{s}-260}{30}\right),
$$

where $t_{s}$ corresponds to the initial development time of a patch and $t$ is the simulation time. The starting times were selected following an exponential distribution with a rate parameter $\Delta^{-1}$

$$
P\left(t_{s}\right)=\frac{e^{-t_{s} / \Delta}}{\Delta}
$$

In all our simulations $\Delta=25 \mathrm{~min}$. The advection velocity $V_{f}$ was selected to be constant along the longest axis of the channel ( $x$-axis) while the $y$-axis dependency was calculated using the Navier-Stokes equation with the assumption of a laminar Pouseuille flow. This gives a flow that is mostly planar with a sharp drop at the boundaries, with a boundary layer of about 50 $\mu \mathrm{m}$, which is of the order of half the height of the channel (see supplementary information). The system was initiated with each patch at its steady state. Different initial states were tested and did not seem to influence the final results, since the system quickly relaxes to its steady state.

We also performed simulations using the two-component version of this model, which makes the assumption that the intracellular production of cAMP is immediately transported to the extracellular medium. This is achieved numerically by setting $\partial_{t} \beta=0$, thus the set of equations becomes

$$
\begin{gathered}
\partial_{t} \gamma=D \nabla^{2} \gamma-v(y) \cdot \nabla \gamma+s \Phi(\rho, \gamma)-k_{e} \gamma, \\
\partial_{t} \rho=k_{1}\left[-f_{1}(\gamma) \rho+f_{2}(\gamma)(1-\rho)\right],
\end{gathered}
$$

where $s=q k_{t} \alpha \sigma /\left(h\left(k_{t}+k_{i}\right)(1+\alpha)\right)$. All parameters used are the same as in Eq 1 .

\section{Results \\ Characterization of the flow-driven waves at high flow rates}

In the absence of flow, signaling $D$. discoideum cells synchronize and show formation and propagation of spiral waves (see supplemental S1 Video). When subjected to advective flows, the spiral patterns are replaced by wave trains traveling downstream. Fig $2 \mathrm{~d}$ shows an example of flow-driven waves for an average flow velocity of $V_{f}=10 \mathrm{~mm} / \mathrm{min}$. The image contrast reflects the shape changes of the cells. The light bands correspond to high concentrations of cAMP and consist of elongated cells while in the dark bands the cAMP concentrations is small and cells remain round [12, 30-32].The corresponding space-time plot is shown in Fig 2c, where light intensity is averaged over the $2 \mathrm{~mm}$ width of the channel and then stacked up along the time axis. The slope of the diagonal bands give the inverse of the average propagation velocity of the waves along the channel. The wave shape and propagation speed strongly depend on the strength of the imposed flow velocity. At small flow rates, a wave train develops spontaneously that fills the whole length of the channel (Fig 3a and supplementary S2 Video). The wavelength of the traveling waves increases linearly with the imposed flow velocity [15] and becomes comparable or larger than the length of the microfluidic channel at high flow rates (Fig $3 b-3 d$ ). Deformations of the wave front also increase significantly with the imposed flow velocity. Planar wave fronts at small flow rates deform to parabolic fronts at intermediate velocities and become extremely extended at higher flow speeds (Fig 3b-3d, and supplementary S3, S4 and 


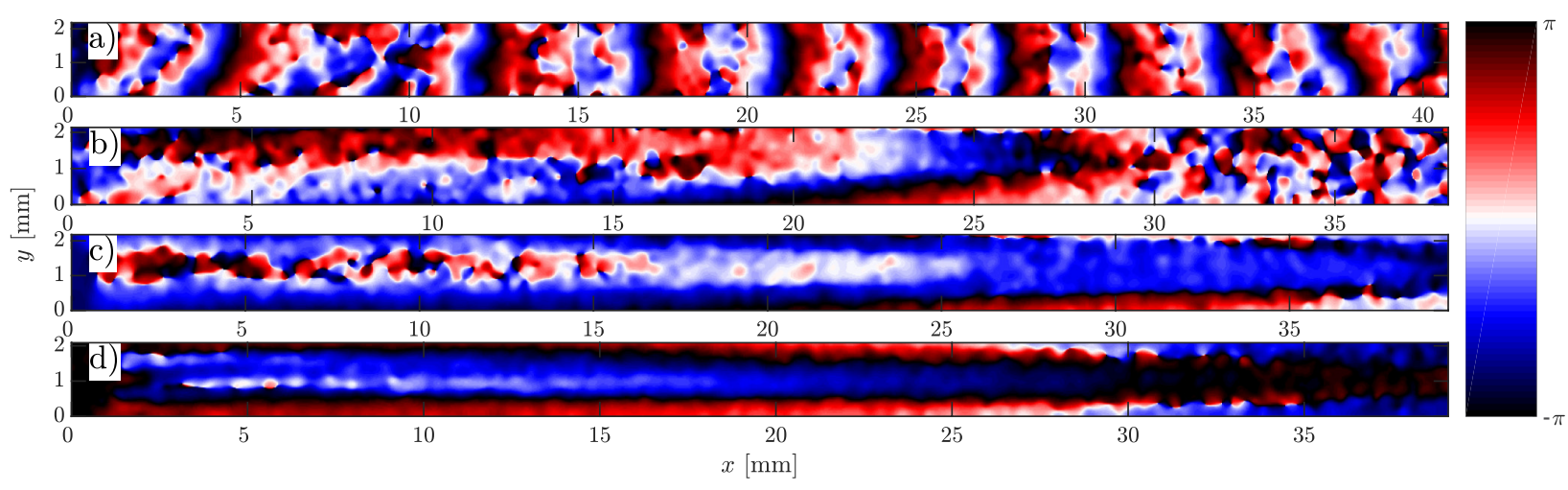

Fig 3. Phase map of the flow-driven waves showing different wave shapes at different imposed flow velocities. a) Practically planar wave fronts at $V_{f}=0.5 \mathrm{~mm} / \mathrm{min}$. b) Parabolic shape at $V_{f}=5 \mathrm{~mm} / \mathrm{min}$. Extremely elongated parabolic wave fronts at flow velocities of $V_{f}=10 \mathrm{~mm} / \mathrm{min}$ and $V_{f}=15$ $\mathrm{mm} / \mathrm{min}$ are shown in c) and d), respectively.

\section{https://doi.org/10.1371/journal.pone.0194859.g003}

S5 Videos). Notice that the wave shape does not reflect the flow profile which is relatively constant across the width of the channel and drops quickly to zero at a length scale comparable to half of the channel's height $(50 \mu \mathrm{m})$, see supplementary S1 Fig and Ref. [29] for detailed calculations of the flow profile. Moreover, at small and intermediate flow rates, the waves propagate solely in the flow direction. However, at higher flow rates they propagate both in the flow direction as well as transversal to the imposed flow. While propagation speed along the flow $\left(v_{\|}\right)$is comparable to the imposed flow velocity, the transversal propagation speed $\left(v_{\perp}\right)$ is much smaller and of the order of the propagation velocity of waves emitted by spirals in this system in the absence of flow $\left(v_{\perp, \text { avg }}=0.45 \pm 0.04 \mathrm{~mm} / \mathrm{min}\right.$ ). The wave period $T$ shows no clear velocity dependence, and takes on a value of $T_{\text {avg }}=5.98 \pm 0.25 \mathrm{~min}$. We also measured the width of the wave fronts $d$, as a function of the imposed flow velocity. We found a linear dependency which is shown in Fig 4d. For this measurement, we calculated the second moment of the light intensity $I$ at the middle of the channel defined as $\sigma^{2}=\sum_{i} I_{i}\left(x_{i}-\bar{x}\right)^{2} / \sum I_{i}$, and multiplied $\sigma$ by the factor of 2.355 to obtain $d$ as the "full width at half maximum" (FWHM) of a Gaussian distribution with standard deviation $\sigma$.

At high flow velocities, the wave generation site plays an important role for its final shape. If the wave is initiated close to the vertical middle of the channel, it propagates along the length and across the width of the channel. Since the wave propagation velocity parallel to the flow is
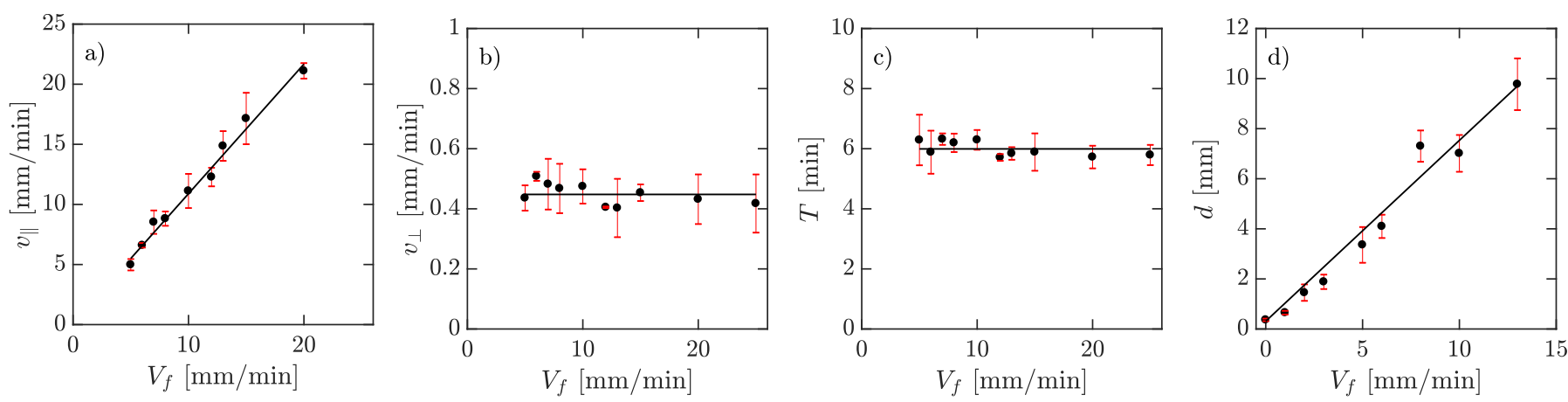

Fig 4. Experimental data on dependency of a) wave speed along the channel $v_{\|}$, b) wave speed in transversal direction $v_{\perp}$, c) wave period $T$, and d) wave front thickness as a function of imposed flow velocity $V_{f}$. Continuous lines represent in a), d) least square fit assuming linear scaling and in b), c) average transversal propagation velocity and wave period.

https://doi.org/10.1371/journal.pone.0194859.g004 


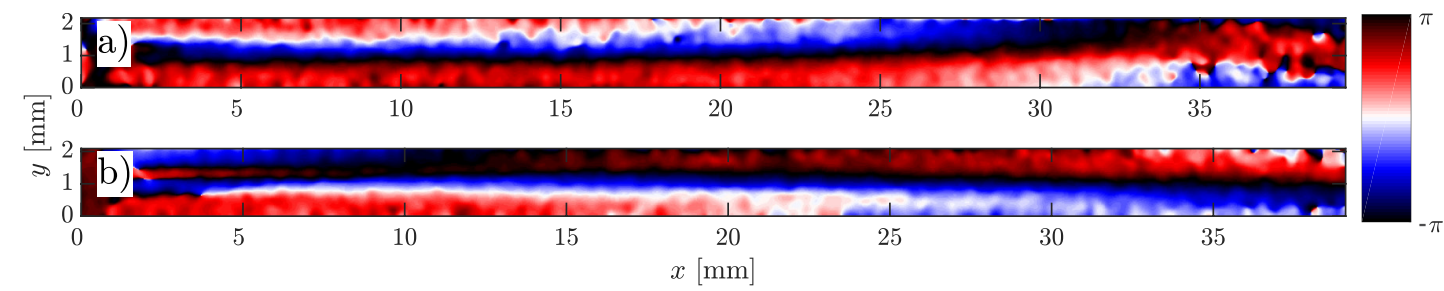

Fig 5. a) A half-parabolic shaped wave front observed at $V_{f}=10 \mathrm{~mm} / \mathrm{min}$. b) Two stripe-like waves initiating at top and bottom boundaries for flow speed of $V_{f}=15 \mathrm{~mm} / \mathrm{min}$.

https://doi.org/10.1371/journal.pone.0194859.g005

much faster than perpendicular to it, the wave gets stretched along the channel. This leads to the formation of an elongated parabolic-shaped wave front (Fig 3c and supplemental S4 Video). However, if the initial excitation is in the vicinity of top $(y=2 \mathrm{~mm})$ or bottom boundaries $(y=0)$, the wave can only propagate in one direction across the channel, which results in a half-parabola wave front, as shown in Fig 5 a and supplemental S4 Video. At very high speeds $\left(V_{f} \geq 15 \mathrm{~mm} / \mathrm{min}\right.$ ), we observe an extreme version of this process where stripe-like patterns form, as shown exemplary in Fig $5 \mathrm{~b}$ for $V_{f}=15 \mathrm{~mm} / \mathrm{min}$ and supplemental S5 Video.

\section{Numerical simulations results}

To study the wave shape in our system in a more detailed manner, we performed numerical simulations of the two-component model (Eq 2) at high flow speeds and fixed parameters $\sigma=$ $0.55 \mathrm{~min}^{-1}, k_{e}=9.5 \mathrm{~min}^{-1}$ (excitable regime). Starting with an initial perturbation centered upstream in the channel, we observed that the produced waves do not acquired a parabolic shape, but rather a planar form very similar to the flow profile applied as it is shown in Fig 6a and supplementary S6 Video. We compared these patterns to simulations of inert particles being advected with the same flow, and found very good agreement between the two as shown in the top two panels of Fig 6 . This direct correspondence between the wavefront evolution and the advection velocity is due to the instantaneous reaction of the cells to the extracellular presence of cAMP.

In contrast to the two-component model, simulations of the three-component model (Eq 1) with the same parameters gave a shape much more similar to the experiments as can be

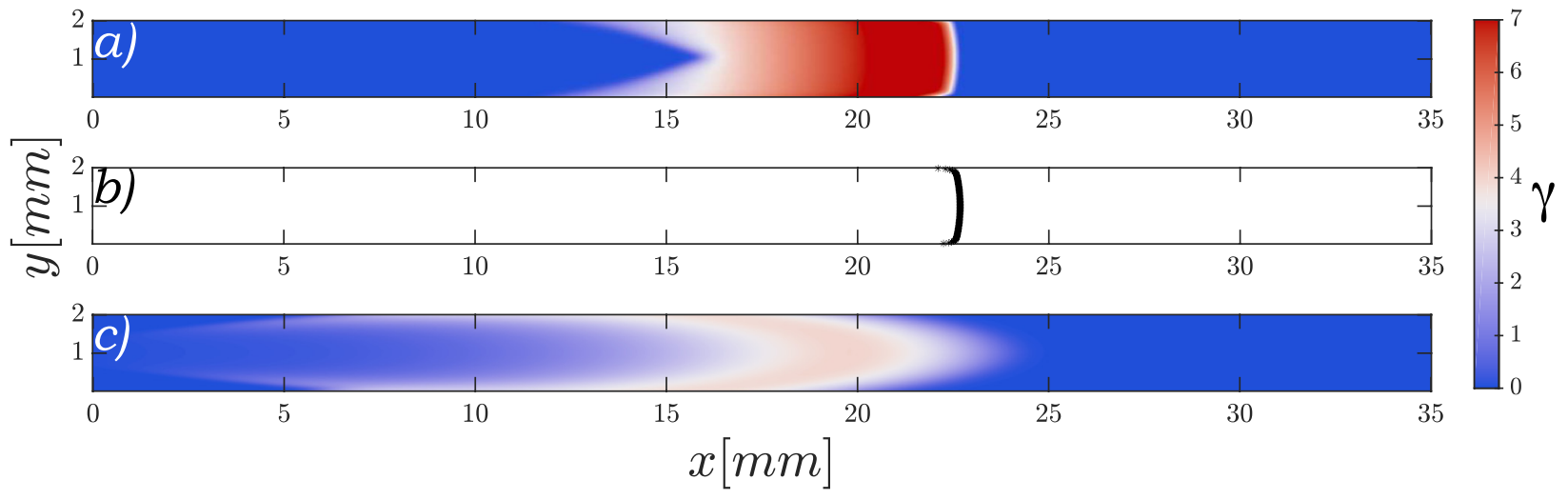

Fig 6. Comparison of wave shapes. For a) the two-component model and c) the three-component model an initial perturbation was applied center upstream on the channel and advected at $V_{f}=10 \mathrm{~mm} / \mathrm{min}$. Panel b) shows a group of particles with no interaction between them starting at the same position as the perturbation in a) and being advected at $V_{f}=v_{\perp}+10 \mathrm{~mm} / \mathrm{min}$, with $v_{\perp}=1.8 \mathrm{~mm} / \mathrm{min}$ the velocity of the two-component model wave without advection.

https://doi.org/10.1371/journal.pone.0194859.g006 

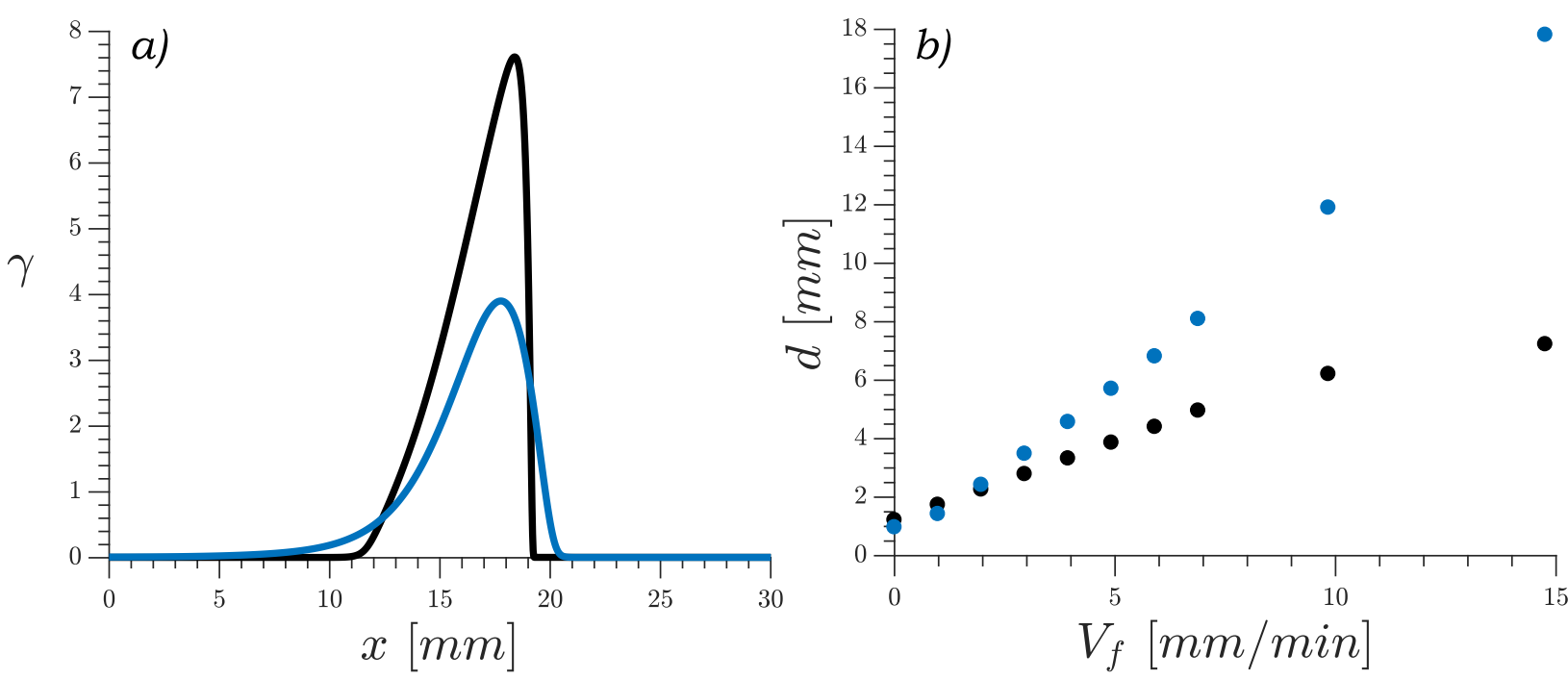

Fig 7. a) Wave profile comparison between the two- and three-component models at imposed flow velocity of $V_{f}=5 \mathrm{~mm} / \mathrm{min}$. b) Wave thickness vs imposed flow for the different models; calculated as 2.355 times the square root of the second moment. Two-component model in black, threecomponent model in blue.

https://doi.org/10.1371/journal.pone.0194859.g007

seen in Fig 6c and supplementary S7 Video. In this version of the model there is a non-instantaneous transport of cAMP between the intracellular and the extracellular media, thus slowing down the waves and effectively shrinking the difference between the velocities across and along the channel, allowing for more rounded shapes. The striking difference between the waves generated by the two models can also be appreciated in the wave profile under advective flow shown in Fig 7a. Here it can be seen that in the fast dynamics model the front of the wave is very sharp, with the cAMP rising to its maximum value very quickly. In the three-component model the wave build up is much slower showing a softer curve that looks more similar to our experimental observations.

We found the velocity of the observed waves to increase linearly with the applied flow for both models, in agreement with the experiments. We also observed an increase of the thickness on the wave profile with increased advection flow. To characterize this, we calculated $2.355 \sigma$, where $\sigma^{2}$ is the second moment of the wave along the middle of the channel defined as $\sigma^{2}=\sum \gamma_{i}\left(x_{i}-\bar{x}\right)^{2} / \sum \gamma_{i}$. These results are shown in Fig $7 \mathrm{~b}$. The increase is faster in the three-component model than in the two-component one, consistent with the profile shown in Fig $7 a$.

Finally, we performed simulations with cells following a developmental path as described in Materials and Methods. Similarly as previously observed in [33], we see cAMP waves starting from cells more advanced in their developmental path, i.e. higher $t_{s}$. When we tried varying the patch size we observed that a minimum amount of cells together in the oscillatory regime were necessary to initiate a wave. For bigger patches, one patch was enough to initiate a wave. Interestingly, we observed that at high speeds (above $2 \mathrm{~mm} / \mathrm{min}$ ) only oscillatory patches at the left end (upstream) of the channel generate waves. Advanced cell clusters down the channel failed to produce waves. The wave shapes observed were of a wide variety, very similar to the ones observed in experiments. The numerical waves presented in Figs 8 and 9 can be compared to the experimental ones of Fig 3, showing very elongated parabolic-shape waves and waves moving perpendicularly to the flow (see supplementary S8 and S9 Videos). 


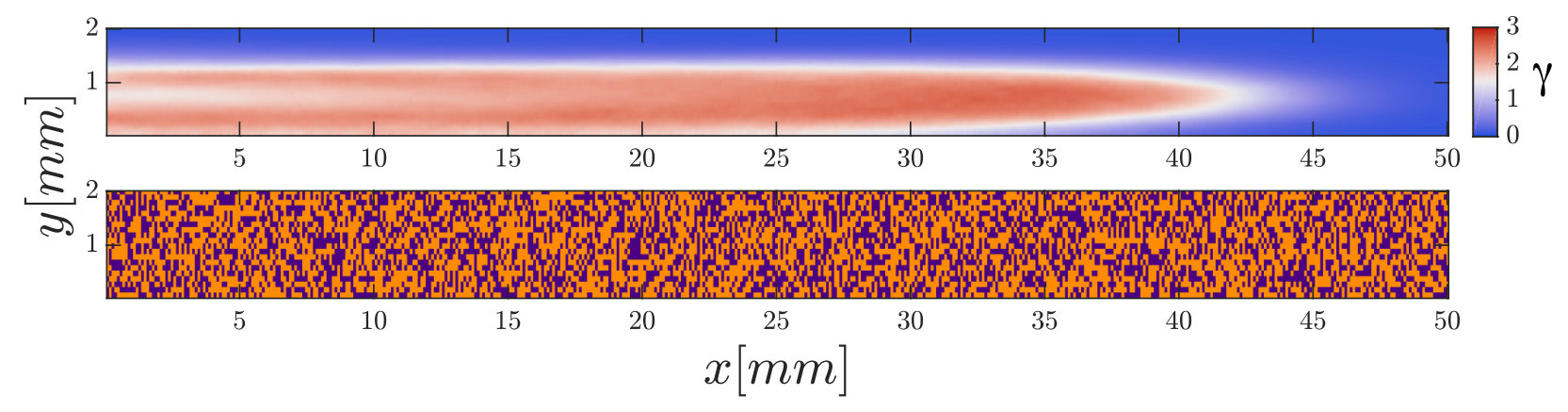

Fig 8. Parabolic-shaped wave observed in simulations using a developmental path. Advecting flow $V_{f}=15 \mathrm{~mm} / \mathrm{min}$. Top: $\mathrm{cAMP}$ concentration. Bottom: State of the cells at the moment of wave initiation: Excitable cells in orange and oscillatory cells in purple. The wave is initiated upstream almost at the middle of the channel.

https://doi.org/10.1371/journal.pone.0194859.g008

\section{On- and off- cycles of the imposed flow}

To verify our assumptions on the dynamical state of the cells in the numerical simulations, we performed experiments in which we abruptly switched off the imposed flow, after the flowdriven waves had been fully established throughout the channel. This lets us to distinguish between real waves of cAMP and phase waves, as both types of waves respond differently to changes in flow rate. If the cells are mostly in the oscillatory regime, we expect the waves to be phase waves. Since a phase wave is not directly induced by the diffusing chemicals, it should travel at the same velocity and width after turning off the flow. In contrast, an excitation (trigger) wave should propagate at the normal speed selected nonlinearly by the reaction-diffusion balance of the system and should also recover its standard width in the absence of the flow. Finally, it is also possible that the waves are not stable under abrupt changes of the flow rate.

Thus, we performed experiments in which we switched off the imposed flow while there were flow-driven waves clearly visible in the channel. The corresponding space-time plot of this experiment is shown in Fig 10. We find that in the presence of an external flow, the waves have a higher amplitude as it can be seen in Fig 10 and supplementary S10 Video. The thickness of the wave fronts becomes two to three times larger in the presence of flow (see Fig 11). For a number of experiments, we observed that the waves in the channel would slow down and travel further along the channel with their typical velocity of $0.4 \mathrm{~mm} / \mathrm{min}$ in the absence of advection, as shown in Fig 11 and S10 Video. However, these waves usually did not traverse the channel very far, being annihilated by emitted waves from newly formed centers. These

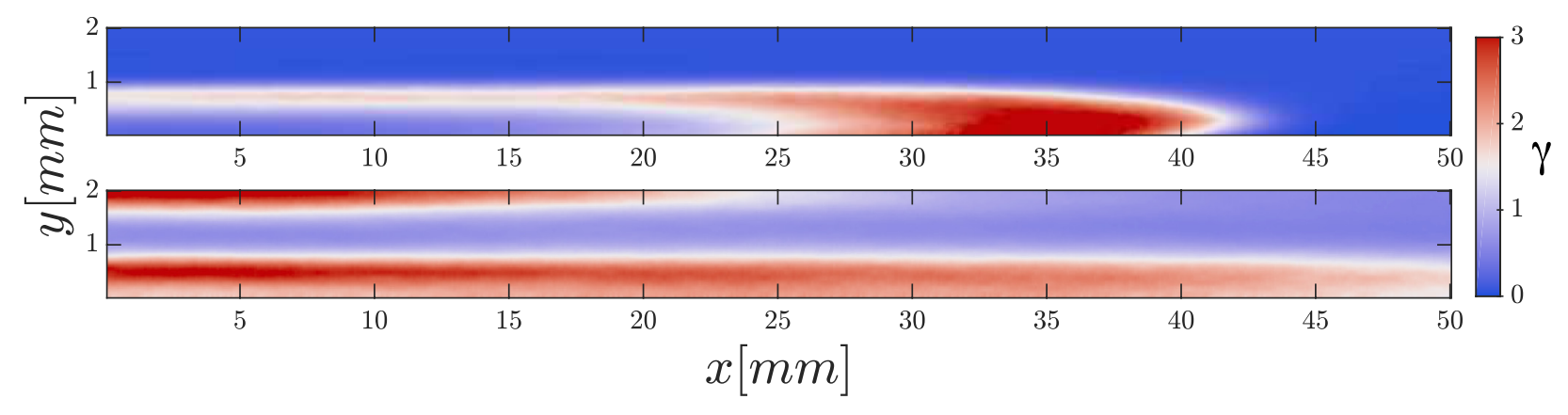

Fig 9. Elongated waves observed in simulations using a developmental path. Advecting flow $V_{f}=15 \mathrm{~mm} / \mathrm{min}$. Top and bottom panels show cAMP waves in simulations with two different initial conditions in the state of the cells.

https://doi.org/10.1371/journal.pone.0194859.g009 


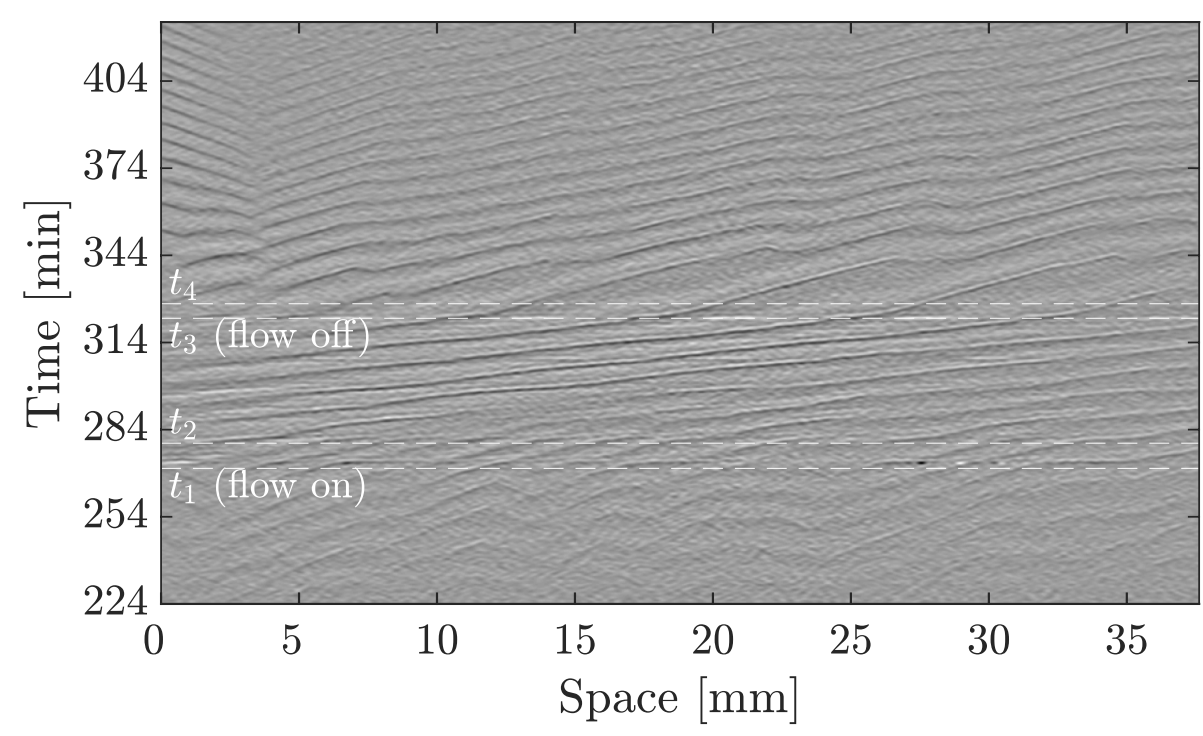

Fig 10. Space-time plot of an experiment in which the flow was initially absent, then turned on $\left(V_{f}=1 \mathrm{~mm} / \mathrm{min}\right)$ at $t_{1}$ and turned off again at $t_{3}$. While the flow is off $\left(t \leq t_{1}\right)$, the cells show target patterns. After it turns on at $t_{1}$, there is a short disordered phase until flow-driven waves fully develop, which travel downstream at $v_{\|, \text {on }}=0.99 \pm 0.03$ $\mathrm{mm} / \mathrm{min}$. At time $t_{3}$, the flow is turned off and the waves still propagate further downstream at slower speed of $v_{\|, \text {off }}=0.37 \pm 0.03 \mathrm{~mm} / \mathrm{min}$ for $30 \mathrm{~min}$. They ultimately vanish on collision with waves emitted from new centers.

https://doi.org/10.1371/journal.pone.0194859.g010

observations confirm that these propagating waves are trigger waves and at least a portion of the cells are in the excitable regime.

We also performed numerical simulations with a similar setup, that is, with a developmental path scheme and switching off the flow once the waves were formed. Results from those simulations are presented as a space-time plot in Fig 12 and supplementary S11 Video. We observed a change in wave thickness and velocity once the flow is switch off, with some waves continuing traveling at a smaller speed, showing good agreement with the experimental observations.

Finally, we increased step-wise the imposed flow velocity to further study the system response. Fig 13 shows the space-time plot of an experiment where the imposed flow increases from $1 \mathrm{~mm} / \mathrm{min}$ to $4 \mathrm{~mm} / \mathrm{min}$ and returns back to $1 \mathrm{~mm} / \mathrm{min}$ at the end. The slope of the
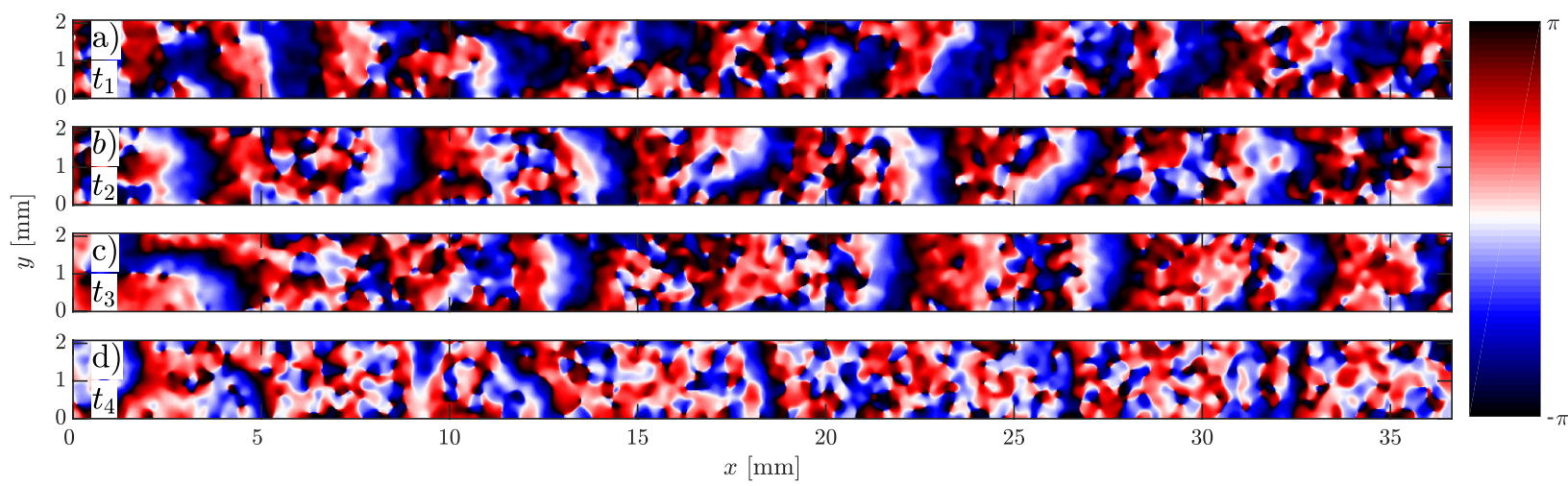

Fig 11. a) The wave pattern at the time point that the flow of magnitude $V_{f}=1 \mathrm{~mm} / \mathrm{min}$ is turned on $\left(t_{1}\right.$ in Fig 10), and b) at time $t_{2}, 8 \mathrm{~min}$ later. c) The fully developed waves shortly before turning off the flow at time $t_{3}$ and $\mathrm{d}$ ) the waves at time $t_{4}$, shortly after switching off the flow.

https://doi.org/10.1371/journal.pone.0194859.g011 


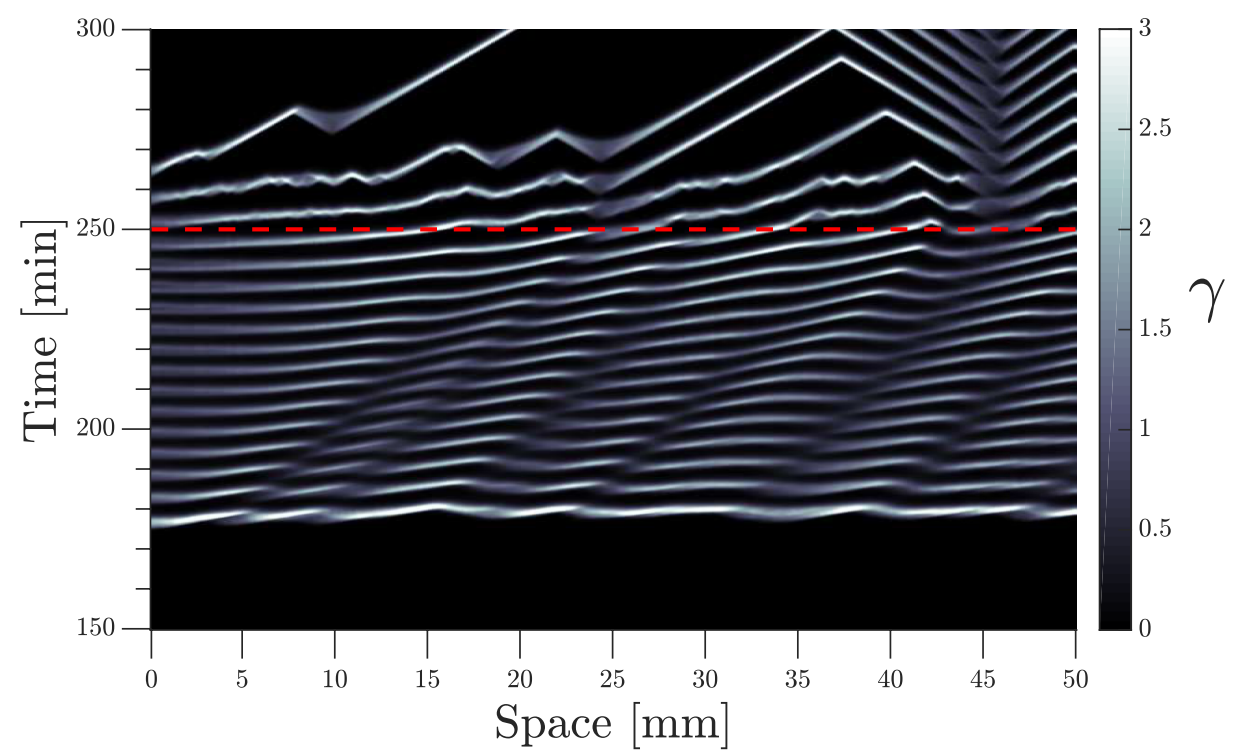

Fig 12. Space-time plot of numerical simulations using the developmental path scheme. The advecting flow is initially $V_{f}=1 \mathrm{~mm} / \mathrm{min}$ and stops at $t=250 \mathrm{~min}$.

https://doi.org/10.1371/journal.pone.0194859.g012

diagonal bands, which give a measure of the inverse wave velocity, follow the velocity jumps of the applied flow (see supplementary S12 Video). Interestingly, we observe a transient decrease in the wave period as the imposed velocity changes from $2 \mathrm{~mm} / \mathrm{min}$ to $3 \mathrm{~mm} / \mathrm{min}$. Since the wavelength is already fixed for the previously developed waves at $2 \mathrm{~mm} / \mathrm{min}$, they adjust to higher speed by decreasing the period to $4 \mathrm{~min}$ (roughly $2 / 3$ of the normal $6 \mathrm{~min}$ period).

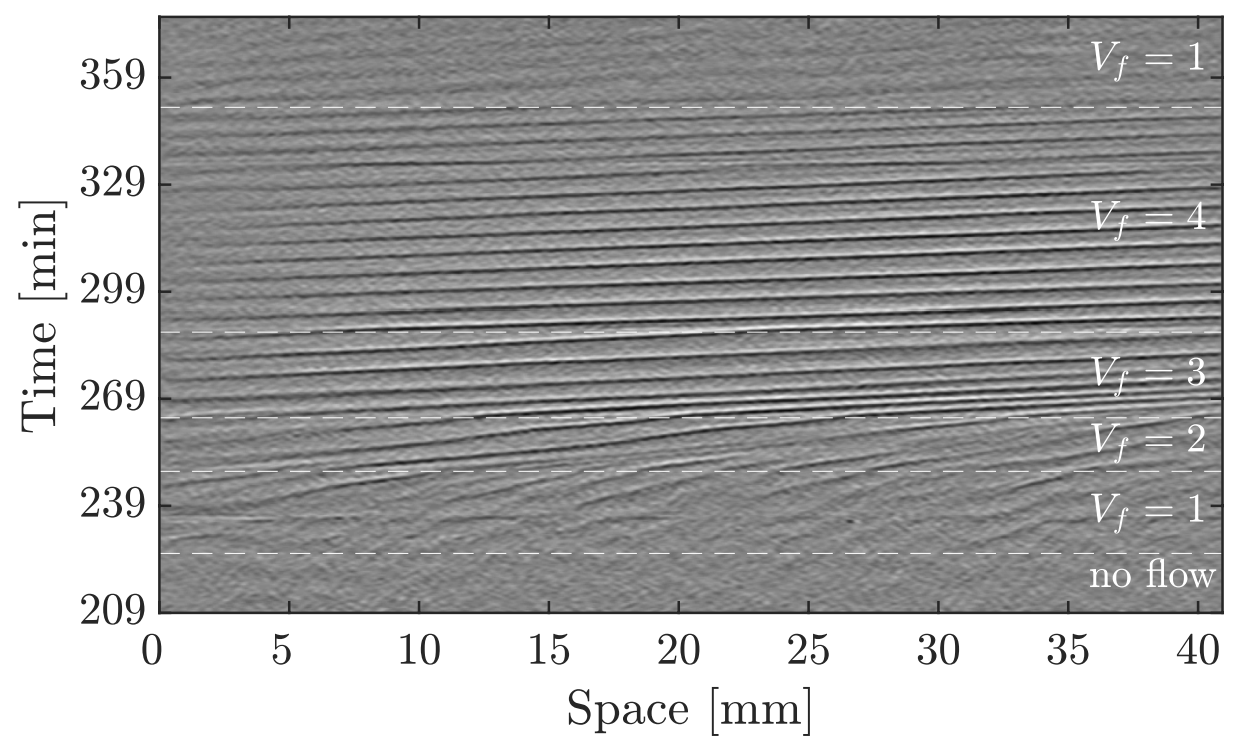

Fig 13. Propagating waves follow the velocity jumps of the imposed flow from $1 \mathrm{~mm} / \mathrm{min}$ to $4 \mathrm{~mm} / \mathrm{min}$. The waves already established at $V_{f}=2 \mathrm{~mm} / \mathrm{min}$ accelerate as the flow increases to $3 \mathrm{~mm} / \mathrm{min}$, and to keep the wavelength already set at $V_{f}=2 \mathrm{~mm} / \mathrm{min}$, period decreases transiently for these waves to $4 \mathrm{~min}$.

https://doi.org/10.1371/journal.pone.0194859.g013 


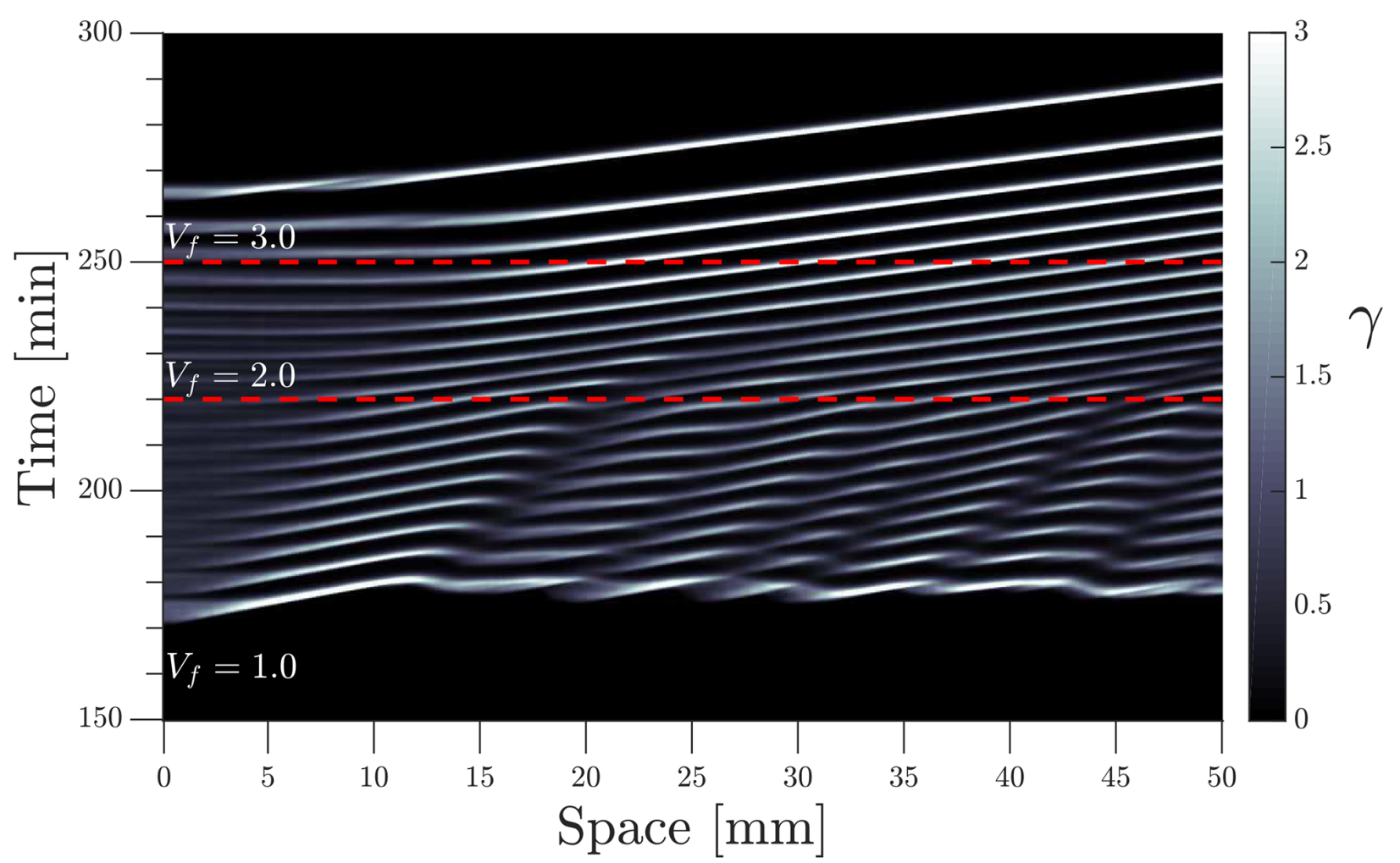

Fig 14. Space-time plot of numerical simulations using the developmental path scheme and a stepwise incremental flow. Initial flow velocity $V_{f}=$ $1.0 \mathrm{~mm} / \mathrm{min}$, incremented at $t=220 \mathrm{~min}$ to $V_{f}=2.0 \mathrm{~mm} / \mathrm{min}$, and again increased to $V_{f}=3.0 \mathrm{~mm} / \mathrm{min}$ at $t=250 \mathrm{~min}$.

https://doi.org/10.1371/journal.pone.0194859.g014

Newly developed waves at the inlet area of the channel ( $\left.V_{f}=3 \mathrm{~mm} / \mathrm{min}\right)$, have a higher wavelength and velocity, and the wave period recovers back to the standard value of $6 \mathrm{~min}$.

Numerical simulations of a similar system with developmental path scheme is shown in Fig 14 and supplementary S13 Video, where the flow velocity is increased stepwise from $1 \mathrm{~mm} /$ $\min$ to $3 \mathrm{~mm} / \mathrm{min}$.

\section{Aggregation under the influence of flow}

To investigate aggregation dynamics of $D$. discoideum cells in the presence of flow, we performed experiments in which the flow is maintained well into the culmination phase of the life cycle. In our experiments, waves appear 3-6 hours after starvation. During this time, chemotactic cell movement is still weak [34] and the variations in cell density are not significant (compare Fig 15a and 15b). Later, 8 hours into starvation, cells form atypical aggregate patterns at high flow rates, as shown in Figs $15 \mathrm{c}$ and 16e. Similar to the experiments in Ref. [35], we observed cone-shaped long streams that existed in the downstream and lateral side of the centers. The lateral streams continue to line up in the direction of the imposed flow (see Fig $15 \mathrm{~d}$ and supplemental S14 Video). Interestingly, the cells upstream the center do not sense any stimulus and aggregate randomly. The length of the long streams are about $4 \mathrm{~mm}$, showing that the stimulus from the centers are extended over a long distance downstream so that only the cells directly downstream of the center will show any orientation. We also used bright field microscopy to closely look at the wave propagation and streaming process under flowing buffer. Snapshots of the cell distribution during the aggregation process are shown in Fig 16. 


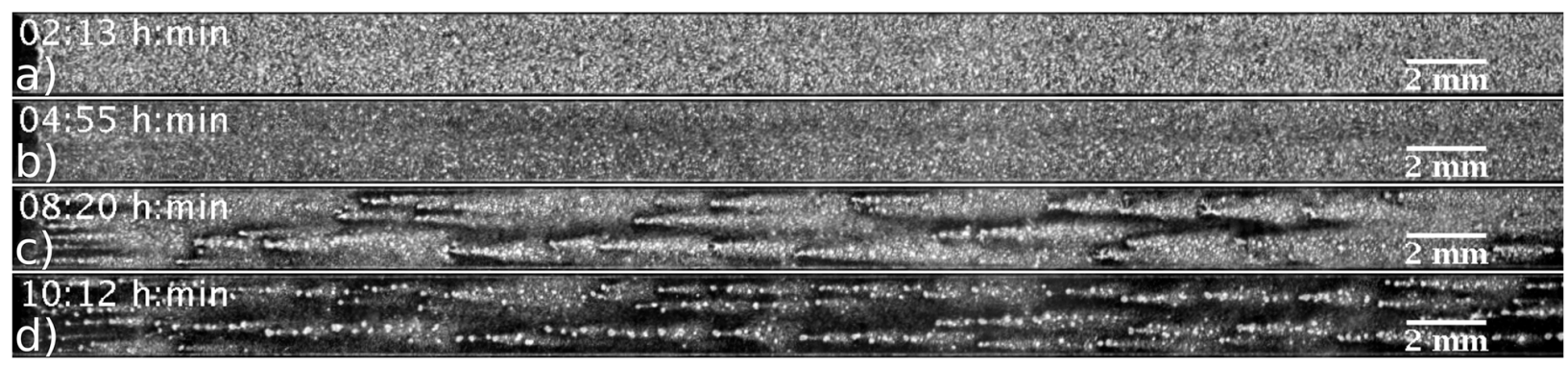

Fig 15. a) Uniform cell distribution at the beginning of experiment in a flow-through microfluidic channel $\left(V_{f}=10 \mathrm{~mm} / \mathrm{min}\right)$. b) During the propagation of the waves, the variations in cell density due to chemotactic cell movement are still negligible. c) Aggregation patterns after 8 hours starvation show cone-shaped structures with long streams downstream of the centers. d) Lateral streams, extended almost $0.5 \mathrm{~mm}$ in $y$-direction, start to line up in the direction of flow.

https://doi.org/10.1371/journal.pone.0194859.g015

In particular, the cone-shaped structures and long stream lines are well visible in Fig 16e and supplementary S15 Video.

\section{Discussion}

We have found that colonies of $D$. discoideum initiate cAMP waves even if subjected to high flow rates (see [15] for results on lower flow rates). The speed of the waves along the channel is

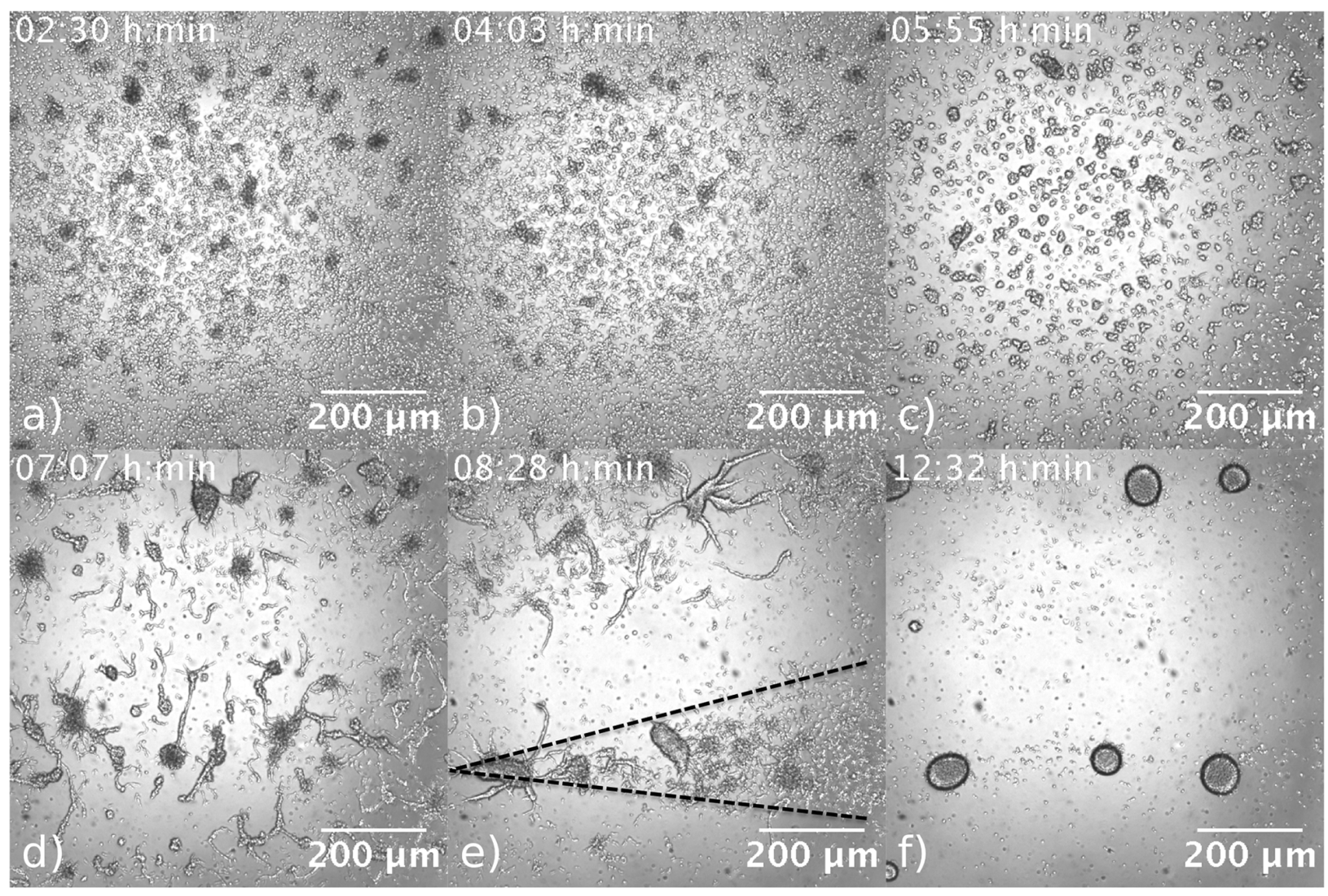

Fig 16. Aggregation process observed in a bright field microscope at $V_{f}=\mathbf{1 0} \mathrm{mm} / \mathrm{min}$. Cone-shaped aggregation domains with long stream lines are visible in panel e).

https://doi.org/10.1371/journal.pone.0194859.g016 
proportional to the imposed flow velocity, while both the velocity in the transversal direction and the wave period are independent of imposed flow velocity. The wave speed in transversal direction seems to be the same as in the spiral waves of $D$. discoideum populations in the absence of flow, where they move at $0.4 \mathrm{~mm} / \mathrm{min}$ [32] [12] and the period found is consistent with the one of target patterns in previous studies of 5-7 $\mathrm{min}$ [32] / 5-8 $\mathrm{min}$ [36]. This is a strong indicator that period and wave speed are intrinsic characteristics of the system that allow for robust aggregation even under strenuous advecting flow conditions. It is interesting that $D$. discoideum cells aggregate even at high imposed flow velocities. The type of trailing edge we observe is similar to the ones previously observed in water flow experiments of Ref. [35].

The shape of the cAMP waves changes with higher imposed flow speeds, transitioning from a planar wave to a parabolic-shaped wave that becomes increasingly longer the higher the flow rate. At high buffer flow rates, the waves are extremely elongated and their observable portion moves mostly perpendicular to the flow. Indeed, we have observed waves that are extended over the entire length of the channel. These waves, just like parabolic-shaped waves, originate at the inlet either the top or the bottom of the channel. These observations are consistent with what we observed in simulations with a developmental path, where clusters of cells advanced on the path would fail to produce a wave unless they are located near the upstream boundary. If the waves are generated upstream bottom (or top) then the cAMP quickly gets advected downstream on a time scale that is much shorter than its degradation time, thus looking like a line of cAMP that moves upwards (downwards) at the usual wave propagation velocity $(\approx 0.4$ $\mathrm{mm} / \mathrm{min})$.

We also observed an increase of the wave intensity and width in the presence of flow both in experiments and simulations. We found wave widths of up to $3 \mathrm{~mm}$, which are wider than the width of spiral waves in the undisturbed system, that have been reported around $0.3-1 \mathrm{~mm}$ [12] for the whole wave, and $0.7 \mathrm{~mm}$ at half height [32]. We understand the increase in width based on previous research showing that D.discoideum emits cAMP during a period of time of approximately $t_{f} \approx 3-4 \mathrm{~min}$ [37] / 1-3 min [32], thus in a first order approximation $d=v_{\|} \cdot t_{f}$ where $d$ is wave thickness, $v_{\|}$wave propagation velocity along the flow, and $t_{f}$ firing time. In other words, due to the flow, the cAMP produced covers a larger distance before the cell gets inactive. The increase in intensity when subjected to advection has also been observed in other reaction-diffusion systems, such as the Beluosov-Zhabotinsky reaction [38], while the increase in thickness has also been reported in numerical simulations of the FitzHugh-Nagumo model [39] and of autocatalytic fronts [40].

The wave initiation process is the most intriguing of the observed effects. We observed that for slow flow velocities the waves can start from anywhere in the system. For faster flows, we believe that the cAMP emitted by the centers is quickly advected away, and therefore do not create a supra-threshold perturbation in neighboring cells that would allow wave propagation. In the experiments, wave formation might be due to unbounded phosphodiesterase, since cells downstream would receive the enzyme secreted by the cells upstream, while the cells at the upper boundary receive a clean flow coming from the injected flow. Since this flow has an effective lower degradation, it allows for the creation of new wave pulses. More experiments using PDE deficient cells are necessary to confirm this hypothesis.

We tested the dynamical state of the cells by abruptly setting the flow velocity to zero and observing the response of the waves. In several experiments we find that the waves traveling along the channel move along it without flow as well. The waves moved at the speed of the imposed flow while the flow was applied and immediately slowed down to approximately the propagation velocity of spiral waves, as the flow was switched off. Furthermore, the width of the waves decreased, as the flow was switched off. This type of response is expected 
in excitable systems where a pulse has defined characteristics like speed and width given by the system to which the wave would return in the absence of flow. In contrast, in an oscillatory system a thicker wave would produce a synchronized (bulk) oscillation in that area, so even though the wave propagating would have a normal thickness, the area perturbed at the moment of the switch off would have a less organized behavior with areas of synchronized oscillations. We reproduced these results by switching off the flow in numerical simulations with developmental path. Even though the results depended weakly on at which point along the path the flow was stopped, they showed waves continuing to travel along the channel and some more disordered waves given by the oscillatory patches. Therefore a mixture of oscillatory and excitable cells managed to reproduce our experimental observations.

To summarize, we find that $D$. discoideum cells initiate defined cAMP waves even under the influence of strong advective flows up to $V_{f}=50 \mathrm{~mm} / \mathrm{min}$. We find that the waves change shape depending on the applied flow velocity, transitioning from planar waves at low speeds, to parabolic shaped waves, whose elongation increases with flow velocity. These wave shapes observed at high speeds were strongly dependent on the location of their initiation point. Since the cells move against cAMP gradients when aggregating, the shape of the cAMP waves have an important role on the aggregation process, in regards to this, it is noteworthy that even without the presence of wave centers such as target centers and spirals, the cells are still capable of aggregation. This also shows the capability of $D$. discoideum to signal even in extremely adverse conditions.

The wave speed in transversal direction $v_{\perp}$ and the period of the waves $T$ showed to be robust system characteristics, being constant for all studied flow speeds. The wave speed along the channel $v_{\|}$and the wave width $d$, however, scale linearly with $V_{f}$. We compare these experimental results to numerical simulations of the system. While a two-component model was sufficient for low flow velocities, we find the extension to the three-component model necessary to reproduce the wave shape at higher flows, showing how fundamental the intracellular dynamic is to produce robust signaling.

Experiments conducted with switching off the advecting flow showed waves that travel along the system changing their velocity and width to recover their unperturbed characteristics, displaying a trigger wave behavior, characteristic of excitable systems.

The observed wave shapes were successfully reproduced using a developmental path which added desynchronization to the system, having some cells in an oscillatory state and some in an excitable one. The upstream cells more advanced in this path became the source of the downstream traveling waves. This scheme also reproduced successfully the flow switching off experiments. We expect our investigations to be crucial to understand signaling of D. discoideum cells in the presence of external flows.

\section{Supporting information}

\section{S1 File. Shear stress calculations.} (PDF)

S1 Fig. Flow profile in the channel. Laminar flow profile inside the microfluidic channel in arbitrary units. a) Cut along the channel center $(y=0)$. b) Cut along half channel height $(z=0)$. (EPS)

S1 Video. Experiment without flow. The movie shows an experiment in the macro-channel without an imposed flow, so at $V_{f}=0$. The three rows of this and later movies show in order: 
i) the original Dark-field images ii) the subtracted and band-pass filtered images and iii) the local phase extracted from the band-pass filtered images.

(MOV)

S2 Video. Experiment with flow. Experiment with a low imposed flow of $V_{f}=0.5 \mathrm{~mm} / \mathrm{min}$ showing a planar wave train.

(MOV)

S3 Video. Experiment with flow. Experiment with an imposed flow of $V_{f}=5 \mathrm{~mm} / \mathrm{min}$ showing parabolic waves.

(MOV)

S4 Video. Experiment with flow. Experiment with an imposed flow of $V_{f}=10 \mathrm{~mm} / \mathrm{min}$ showing strongly elongated parabolic waves.

(MOV)

S5 Video. Experiment with flow. Experiment with an imposed flow of $V_{f}=15 \mathrm{~mm} / \mathrm{min}$ showing extremely elongated parabolic waves.

(MOV)

S6 Video. Simulations. Numerical simulation of the two-component model in the excitable regime with an imposed flow of $V_{f}=10 \mathrm{~mm} / \mathrm{min}$. Initial perturbation applied center upstream.

(MOV)

S7 Video. Simulations. Numerical simulation of the three-component model in the excitable regime with an imposed flow of $V_{f}=10 \mathrm{~mm} / \mathrm{min}$. Initial perturbation applied center upstream.

(MOV)

S8 Video. Simulations. Numerical simulation of the three-component model using a developmental path for the parameters $\sigma$ and $k_{e}$, with an imposed flow of $V_{f}=15 \mathrm{~mm} / \mathrm{min}$. Top: cAMP concentration. Bottom: State of the cells, gray for the stable state, purple for the oscillatory regime, and excitable regime in orange.

(MOV)

S9 Video. Simulations. Numerical simulation of the three-component model using a developmental path for the parameters $\sigma$ and $k_{e}$, with an imposed flow of $V_{f}=15 \mathrm{~mm} / \mathrm{min}$. Top: cAMP concentration. Bottom: State of the cells, gray for the stable state, purple for the oscillatory regime, and excitable regime in orange. (MOV)

S10 Video. On-off flow experiment. Experiment which had no flow initially, at $t_{1}=4 \mathrm{~h} 27$ min a flow of $V_{f}=1 \mathrm{~mm} / \mathrm{min}$ was switched on. The flow was switched off again at $t_{3}=5 \mathrm{~h} 21$ $\min$.

(MOV)

S11 Video. Simulations. Numerical simulation of the three-component model using a developmental path for the parameters $\sigma$ and $k_{e}$, with an imposed flow of $V_{f}=1 \mathrm{~mm} / \mathrm{min}$ until $t=250 \mathrm{~min}$, afterwards the flow is off, $V_{f}=0 \mathrm{~mm} / \mathrm{min}$. Top: cAMP concentration. Bottom: State of the cells, gray for the stable state, purple for the oscillatory regime, and excitable regime in orange.

(MOV) 
S12 Video. Jump-in-flow experiment. Experiment which had no flow initially. At $3 \mathrm{~h} 46 \mathrm{~min}$ a flow of $V_{f}=1 \mathrm{~mm} / \mathrm{min}$ was switched on. Next the flow was increased first to $V_{f}=2 \mathrm{~mm} / \mathrm{min}$ at $4 \mathrm{~h} 09 \mathrm{~min}$, then to $V_{f}=3 \mathrm{~mm} / \mathrm{min}$ at $4 \mathrm{~h} 24 \mathrm{~min}$. Finally, the speed was increased to $V_{f}=4$ $\mathrm{mm} / \mathrm{min}$ at $4 \mathrm{~h} 48 \mathrm{~min}$. At $5 \mathrm{~h} 51 \mathrm{~min}$ the flow was reduced to $V_{f}=1 \mathrm{~mm} / \mathrm{min}$ again.

$(\mathrm{MOV})$

S13 Video. Simulations. Numerical simulation of the three-component model using a developmental path for the parameters $\sigma$ and $k_{e}$, with an imposed flow of $V_{f}=1 \mathrm{~mm} / \mathrm{min}$ until $t=220 \mathrm{~min}$, afterwards the flow is increased to $V_{f}=2 \mathrm{~mm} / \mathrm{min}$ and at $t=250 \mathrm{~min}$ increased to $V_{f}=3 \mathrm{~mm} / \mathrm{min}$. Top: cAMP concentration. Bottom: State of the cells, gray for the stable state, purple for the oscillatory regime, and excitable regime in orange.

(MOV)

S14 Video. Aggregation experiment. Experiment with $V_{f}=10 \mathrm{~mm} / \mathrm{min}$ showing original Dark-field images for a longer time to show the aggregation phase of the cells.

(MOV)

S15 Video. Bright-field experiment. Bright-field microscopy experiment showing the aggregation in a channel with $V_{f}=10 \mathrm{~mm} / \mathrm{min}$ (4X magnification).

$(\mathrm{MOV})$

\section{Acknowledgments}

The authors thank M. S. Müller and K. Gunkel for their cheerful help with the preparation of the cells. T. E. acknowledges Deutsche Forschungsgemeinschaft (DFG), project number GH 184/1-1. E.V.H. thanks the Deutsche Akademische Austauschdienst (DAAD), Research Grants-Doctoral Programs in Germany. A.G. acknowledges MaxSynBio Consortium, which is jointly funded by the Federal Ministry of Education and Research of Germany and the Max Planck Society.

\section{Author Contributions}

Formal analysis: Torsten Eckstein, Estefania Vidal-Henriquez.

Investigation: Torsten Eckstein, Estefania Vidal-Henriquez, Albert Bae, Vladimir Zykov, Eberhard Bodenschatz, Azam Gholami.

Supervision: Azam Gholami.

Writing - original draft: Torsten Eckstein, Estefania Vidal-Henriquez, Azam Gholami.

Writing - review \& editing: Torsten Eckstein, Estefania Vidal-Henriquez, Albert Bae, Vladimir Zykov, Eberhard Bodenschatz, Azam Gholami.

\section{References}

1. Rovinsky AB, Menzinger M. Chemical instability induced by a differential flow. Physical Review Letters. 1992; 69(8):1193. https://doi.org/10.1103/PhysRevLett.69.1193 PMID: 10047151

2. Rovinsky $A B$, Menzinger $M$. Self-organization induced by the differential flow of activator and inhibitor. Physical Review Letters. 1993; 70(6):778. https://doi.org/10.1103/PhysRevLett.70.778 PMID: 10054201

3. Wu XG, Nakata S, Menzinger M, Rovinsky A. Differential flow instability in tubular flow reactor: Its convective nature. The Journal of Physical Chemistry. 1996; 100(39):15810-15814. https://doi.org/10. 1021/jp9603546

4. Turing AM. The chemical basis of morphogenesis. Philosophical Transactions of the Royal Society of London B: Biological Sciences. 1952; 237(641):37-72. https://doi.org/10.1098/rstb.1952.0012 
5. Malchow $\mathrm{H}$. Flow-and locomotion-induced pattern formation in nonlinear population dynamics. Ecological Modelling. 1995; 82(3):257-264. https://doi.org/10.1016/0304-3800(94)00095-Y

6. Malchow H. Motion instabilities in prey-predator systems. Journal of Theoretical Biology. 2000; 204:639-647. https://doi.org/10.1006/jtbi.2000.2074 PMID: 10833362

7. Malchow H. Non-equilibrium spatio-temporal patterns in models of non-linear plankton dynamics. Freshwater Biology. 2000; 45(2):239-251. https://doi.org/10.1046/j.1365-2427.2000.00550.x

8. Scheffer M. Should we expect strange attractors behind plankton dynamics-and if so, should we bother? Journal of Plankton Research. 1991; 13(6):1291-1305.

9. Kærn M, Menzinger M, Satnoianu R, Hunding A. Chemical waves in open flows of active media: their relevance to axial segmentation in biology. Faraday discussions. 2002; 120:295-312. https://doi.org/ 10.1039/b103244p

10. Pálsson E, Cox EC. Origin and evolution of circular waves and spirals in Dictyostelium discoideum territories. Proceedings of the National Academy of Sciences. 1996; 93(3):1151-1155.

11. Lee KJ, Cox EC, Goldstein RE. Competing Patterns of Signaling Activity in Dictyostelium Discoideum Phys Rev Lett. 1996; 76:1174-1177. https://doi.org/10.1103/PhysRevLett.76.1174 PMID: 10061652

12. Tomchik K, Devreotes P. Adenosine 3',5'-monophosphate waves in Dictyostelium discoideum: a demonstration by isotope dilution-fluorography. Science. 1981; 212(4493):443-446. https://doi.org/10. 1126/science.6259734 PMID: 6259734

13. Kessler DA, Levine H. Pattern formation in Dictyostelium via the dynamics of cooperative biological entities. Phys Rev E. 1993; 48:4801-4804. https://doi.org/10.1103/PhysRevE.48.4801

14. Mosley MP. Subsurface flow velocities through selected forest soils, South Island, New Zealand. Journal of hydrology. 1982; 55(1-4):65-92. https://doi.org/10.1016/0022-1694(82)90121-4

15. Gholami A, Steinbock O, Zykov V, Bodenschatz E. Flow-Driven Waves and Phase-Locked Self-Organization in Quasi-One-Dimensional Colonies of Dictyostelium discoideum. Physical review letters. 2015; 114(1):018103. https://doi.org/10.1103/PhysRevLett.114.018103 PMID: 25615506

16. Gholami A, Steinbock $O$, Zykov V, Bodenschatz E. Flow-driven instabilities during pattern formation of Dictyostelium discoideum. New Journal of Physics. 2015; 17(6):063007. https://doi.org/10.1088/13672630/17/6/063007

17. Gholami A, Zykov V, Steinbock $O$, Bodenschatz E. Flow-driven two-dimensional waves in colonies of Dictyostelium discoideum. New Journal of Physics. 2015; 17(9):093040. https://doi.org/10.1088/13672630/17/9/093040

18. Martiel JL, Goldbeter A. A model based on receptor desensitization for cyclic AMP signaling in Dictyostelium cells. Biophysical journal. 1987; 52(5):807. https://doi.org/10.1016/S0006-3495(87)83275-7 PMID: 19431710

19. Lauzeral J, Halloy J, Goldbeter A. Desynchronization of cells on the developmental path triggers the formation of spiral waves of cAMP during Dictyostelium aggregation. Proceedings of the National Academy of Sciences. 1997; 94(17):9153-9158. https://doi.org/10.1073/pnas.94.17.9153

20. Parent CA, Devreotes PN. Molecular Genetics of Signal Transduction in Dictyostelium. Annual Review of Biochemistry. 1996; 65(1):411-440. https://doi.org/10.1146/annurev.bi.65.070196.002211 PMID: 8811185

21. Whitesides GM, Ostuni E, Takayama S, Jiang X, Ingber DE. Soft lithography in biology and biochemistry. Annual review of biomedical engineering. 2001;3(1):335-373. https://doi.org/10.1146/annurev. bioeng.3.1.335 PMID: 11447067

22. Décavé E, Rieu D, Dalous J, Fache S, Bréchet Y, Fourcade B, et al. Shear flow-induced motility of Dictyostelium discoideum cells on solid substrate. Journal of cell science. 2003; 116(21):4331-4343. https://doi.org/10.1242/jcs.00726 PMID: 12966168

23. Edelstein AD, Tsuchida MA, Amodaj N, Pinkard H, Vale RD, Stuurman N. Advanced methods of microscope control using $\mu$ Manager software. Journal of biological methods. 2014; 1(2). https://doi.org/10. 14440/jbm.2014.36 PMID: 25606571

24. Siegert F, Weijer CJ. Spiral and concentric waves organize multicellular Dictyostelium mounds. Current Biology. 1995; 5(8):937-943. https://doi.org/10.1016/S0960-9822(95)00184-9 PMID: 7583152

25. Egolf DA, Melnikov IV, Bodenschatz E. Importance of local pattern properties in spiral defect chaos. Physical Review Letters. 1998; 80(15):3228. https://doi.org/10.1103/PhysRevLett.80.3228

26. Tyson JJ, Alexander KA, Manoranjan V, Murray J. Spiral waves of cyclic AMP in a model of slime mold aggregation. Physica D: Nonlinear Phenomena. 1989; 34(1-2):193-207. https://doi.org/10.1016/01672789(89)90234-0

27. Merson R. An operational method for the study of integration processes. In: Proc. Symp. Data Processing; 1957. p. 1-25. 
28. Hundsdorfer W, Verwer JG. Numerical solution of time-dependent advection-diffusion-reaction equations. vol. 33. Springer Science \& Business Media; 2013.

29. Vidal-Henriquez E, Zykov V, Bodenschatz E, Gholami A. Convective instability and boundary driven oscillations in a reaction-diffusion-advection model. Chaos: An Interdisciplinary Journal of Nonlinear Science. 2017; 27(10):103110. https://doi.org/10.1063/1.4986153

30. Alcantara F, Monk M. Signal propagation during aggregation in the slime mould Dictyostelium discoideum. Microbiology. 1974; 85(2):321-334.

31. Gross J, Peacey M, Trevan D. Signal emission and signal propagation during early aggregation in Dictyostelium discoideum. Journal of cell science. 1976; 22(3):645-656. PMID: 1035221

32. Devreotes PN, Potel MJ, MacKay SA. Quantitative analysis of cyclic AMP waves mediating aggregation in Dictyostelium discoideum. Developmental Biology. 1983; 96(2):405-415. https://doi.org/10.1016/ 0012-1606(83)90178-1 PMID: 6299820

33. Geberth D, Hütt MT. Predicting the distribution of spiral waves from cell properties in a developmentalpath model of Dictyostelium pattern formation. PLoS computational biology. 2009; 5(7):e1000422. https://doi.org/10.1371/journal.pcbi.1000422 PMID: 19593362

34. Wessels D, Brincks R, Kuhl S, Stepanovic V, Daniels KJ, Weeks G, et al. RasC plays a role in transduction of temporal gradient information in the cyclic-AMP wave of Dictyostelium discoideum. Eukaryotic Cell. 2004; 3(3):646-662. https://doi.org/10.1128/EC.3.3.646-662.2004 PMID: 15189986

35. Bonner JT, Savage LJ. Evidence for the formation of cell aggregates by chemotaxis in the development of the slime mold Dictyostelium discoideum. Journal of Experimental Zoology. 1947; 106(1):1-26. https://doi.org/10.1002/jez.1401060102 PMID: 20268085

36. Siegert F, Weijer C. Digital image processing of optical density wave propagation in Dictyostelium discoideum and analysis of the effects of caffeine and ammonia. Journal of Cell Science. 1989; 93(2):325335.

37. Van Haastert PJ. A method for studying cAMP-relay in Dictyostelium discoideum: the effect of temperature on cAMP-relay. Microbiology. 1984; 130(10):2559-2564. https://doi.org/10.1099/00221287-13010-2559

38. Kærn M, Menzinger M. Propagation of excitation pulses and autocatalytic fronts in packed-bed reactors The Journal of Physical Chemistry B. 2002; 106(14):3751-3758. https://doi.org/10.1021/jp013541z

39. Ermakova EA, Shnol EE, Panteleev MA, Butylin AA, Volpert V, Ataullakhanov FI. On propagation of excitation waves in moving media: The FitzHugh-Nagumo model. PloS One. 2009; 4(2):e4454. https:// doi.org/10.1371/journal.pone.0004454 PMID: 19212435

40. Leconte M, Martin J, Rakotomalala N, Salin D, Yortsos Y. Mixing and reaction fronts in laminar flows. The Journal of chemical physics. 2004; 120(16):7314-7321. https://doi.org/10.1063/1.1665555 PMID: 15267641 


\section{Shear stress}

To calculate the shear stress acting on the cells in the microfluidic channel, we solve the incompressible Navier-Stokes equation in 3D. The channel has a rectangular geometry $(x \in[0, L], y \in[-c, c], z \in[-b, b])$ and we use zero velocity boundary conditions at the boundaries of the channel cross section $(u(y= \pm c)=0$ and $u(z= \pm b)=0)$,

$$
\rho \frac{D u}{D t}=\rho g-\nabla p+\mu \nabla^{2} u
$$

Here $\mu$ denotes fluid viscosity, $\rho$ fluid density, $p$ pressure, and $u$ fluid velocity. We solve using variable separation and Fourier series, and find an expression for the fluid velocity $u$

$$
\begin{aligned}
u & =\frac{G\left(b^{2}-z^{2}\right)}{2 \mu}+\sum_{n \text { odd }} A_{n} \cosh \left(\frac{n \pi}{2 b} y\right) \cos \left(\frac{n \pi}{2 b} z\right), \quad \text { with } \\
A_{n} & =-\frac{16 G b^{2}}{\mu \pi^{3} n^{3}} \frac{\sin \left(\frac{n \pi}{2}\right)}{\cosh \left(\frac{n \pi c}{2 b}\right)} .
\end{aligned}
$$

Where $G$ denotes the pressure drop in the channel. For a detailed derivation of the velocity please see [1].

We now calculate the wall shear stress from the velocity profile, using the expression

$$
\tau=\left.\mu \partial_{z} u\right|_{z=-c}
$$

and the velocity space derivative

$$
\partial_{z} u=-\frac{G z}{\mu}-\frac{n \pi}{2 b} \sum_{n \text { odd }} A_{n} \cosh \left(\frac{n \pi}{2 b} y\right) \sin \left(\frac{n \pi}{2 b} z\right) .
$$

Considering we do not have access to the pressure drop $G$ across the channel, but only the mean flow $Q$ we find an expression for the mean flow by integrating over the channel cross section,

$$
Q=\frac{G b^{3} c}{\mu}\left[\frac{4}{3}-\frac{8 b}{c} \sum_{n \text { odd }}\left(\frac{2}{n \pi}\right)^{5} \tanh \left(\frac{n \pi c}{2 b}\right)\right],
$$

thus we obtain the pressure drop $G$

$$
G=\frac{Q \mu}{b^{3} c}\left[\frac{4}{3}-\frac{8 b}{c} \sum_{n \text { odd }}\left(\frac{2}{n \pi}\right)^{5} \tanh \left(\frac{n \pi c}{2 b}\right)\right]^{-1} .
$$

We can simplify $G$ by using the values for $c$ and $b$. Note also that since $\pi c /(2 b) \approx 31.4$ the hyperbolic tangent becomes $\tanh (\pi c /(2 b)) \approx 1$, this leads to

$$
\begin{aligned}
G & =\frac{Q \mu}{b^{3} c} \times\left[\frac{4}{3}-\epsilon\right]^{-1} \quad \text { with } \\
\epsilon & =\frac{8 b}{c} \sum_{n \text { odd }}\left(\frac{2}{n \pi}\right)^{5} \tanh \left(\frac{n \pi c}{2 b}\right) \approx 4.2 \times 10^{-2} .
\end{aligned}
$$




\section{snow -}

\begin{tabular}{l|c}
$V_{f}\left[\frac{\mathrm{mm}}{\min }\right]$ & $\tau[\mathrm{Pa}]$ \\
\hline 1 & 0.000918 \\
5 & 0.004590 \\
10 & 0.009180 \\
15 & 0.013771 \\
20 & 0.018361 \\
30 & 0.027541 \\
50 & 0.045902
\end{tabular}

Table 1. Shear stress $\tau$ calculated as a function of imposed flow velocity $V_{f}$, using $\mu=8.9 \cdot 10^{-4} \mathrm{~Pa} \mathrm{~s}$, the dynamic viscosity of water and the channel dimensions $c=1$ $\mathrm{mm}$ and $b=0.05 \mathrm{~mm}$.

Then we can approximate this for small $\epsilon$

$$
G \approx \frac{Q \mu}{b^{3} c}\left[\frac{3}{4}+\left(\frac{3}{4}\right)^{2} \epsilon\right] .
$$

Since we want to find the maximum stress, we choose $y$ to be at the middle of the channel $(y=0)$. Thus, we can simplify $\tau$ as well, $\operatorname{since} \cosh (n \pi y / 2 b)=1$, and $A_{n} \approx 0$ $\forall n \in \mathbb{N}$,

$$
\tau_{y=0}=-G z=-\frac{Q \mu z}{b^{3} c} \times\left[\frac{3}{4}+\left(\frac{3}{4}\right)^{2} \epsilon\right] .
$$

We can now calculate the shear stress for the imposed mean flow velocities used in our experiments. An overview is shown in Table 1.

Thus, the shear stresses are well below the critical value for the onset of mechanosensivity $0.7 \mathrm{~Pa}$ or even detachment 2.6 $\mathrm{Pa}[2]$.

\section{References}

1. Vidal-Henriquez E, Zykov V, Bodenschatz E, Gholami A. Convective instability and boundary driven oscillations in a reaction-diffusion-advection model. Chaos: An Interdisciplinary Journal of Nonlinear Science. 2017;27(10):103110. doi:10.1063/1.4986153.

2. Décavé E, Rieu D, Dalous J, Fache S, Bréchet Y, Fourcade B, et al. Shear flow-induced motility of Dictyostelium discoideum cells on solid substrate. Journal of cell science. 2003;116(21):4331-4343. doi:10.1242/jcs.00726. 

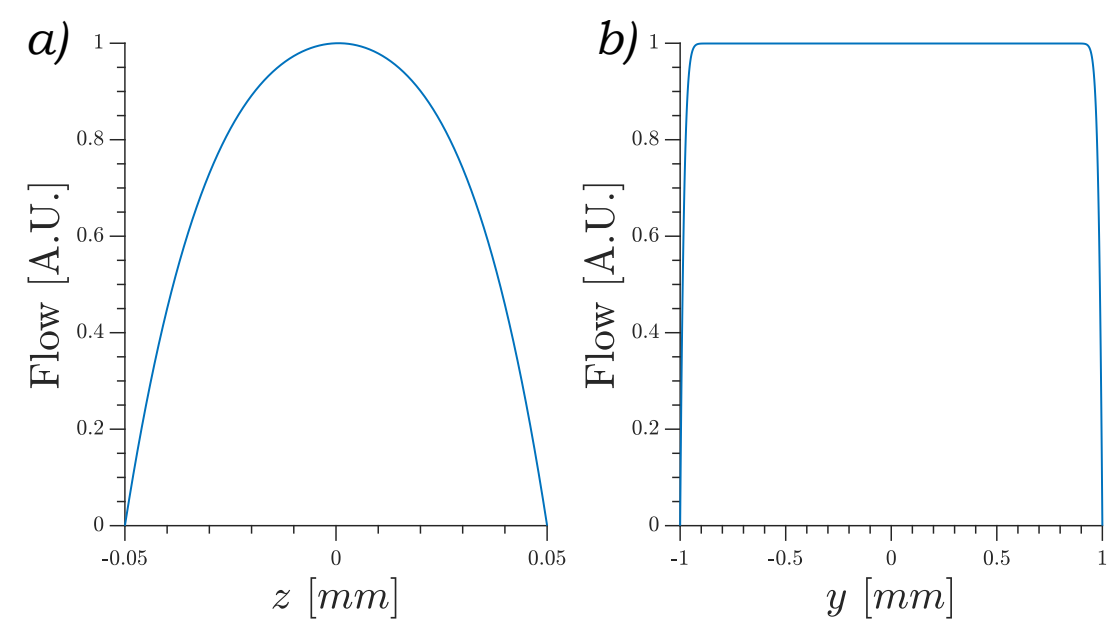

Figure 3.1: Supplementary Figure 1 included with Influence of fast advective flows on pattern formation of Dictyostelium discoideum electronically available. Original caption "Flow profile in the channel. Laminar flow profile inside the microfluidic channel in arbitrary units. a) Cut along the channel center $(\mathrm{y}=0) . \mathrm{b})$ Cut along half channel height $(\mathrm{z}=0) "[76]$. 


\section{Spatial Heterogeneities Shape Collective Behavior of Signaling Amoeboid Cells}

The following chapter is a paper draft by Eckstein, T., Vidal-Henriquez, E., Bae, A., \& Gholami, A. It is currently under review in a peer-reviewed journal.

EVH designed and performed the numerical simulations and analysis. EVH, TE, and AG wrote the manuscript. All authors analyzed data, reviewed and edited the manuscript. Figures 7, 8, S4 to S11, and S13 by EVH. Also by EVH are the electronically available Supplementary Movies 9 to 16, Movie 21, and Movie 22. 


\title{
Spatial heterogeneities shape collective behavior of signaling amoeboid cells
}

\author{
Torsten Eckstein $^{\mathrm{a}, 1}$, Estefania Vidal-Henriquez ${ }^{\mathrm{a}, 1}$, Albert J. Bae ${ }^{\mathrm{a}}$, and Azam Gholami ${ }^{\mathrm{a}, 2}$ \\ ${ }^{a}$ Max Planck Institute for Dynamics and Self-Organization, Am Fassberg 17, 37077 Göttingen, Germany \\ This manuscript was compiled on February 11, 2019
}

We present novel experimental results on pattern formation of signaling Dictyostelium discoideum amoeba in the presence of a periodic array of millimeter-sized pillars. In our experiments with caffeine treated cells, we observe concentric CAMP waves that initiate almost synchronously at the pillars and propagate outwards. These waves have a higher frequency than the other firing centers and dominate the system dynamics. The cells respond chemotactically to these circular waves and stream towards the pillars, forming periodic domains that reflect the periodicity of the underlying lattice. We performed comprehensive numerical simulations of a reaction-diffusion model to study the role of caffeine and characteristics of the boundary conditions given by the obstacles. Our simulations show that a critical minimum accumulation of cAMP around the obstacles is required for the pillars to act as the wave source. This critical value depends on the cAMP production rate, a variable which we can experimentally decrease by adding caffeine. Moreover, our simulations reveal that caffeine reduces the excitability threshold of the cells and increases the sensitivity to CAMP accumulation around the obstacles. In the absence of caffeine, non-treated cells are less sensitive to cAMP accumulation around the pillars and ignore them, as observed in our experiments. Our results suggest that in nature the excitability threshold of the cells is tuned by an adaptation process that optimizes the sensitivity to waves while ignoring the cAMP accumulations around spatial heterogeneities which can interrupt the development process of the cells in complex environments.

pattern formation | excitable media | Dictyostelium discoideum | reaction-diffusion systems

A fundamental process occurring in reaction-diffusion excitable systems is the propagation of nonlinear waves (14). Examples of such waves include chemical waves in the Belousov-Zhabotinsky reaction (5), waves of CO oxidation on Pt catalytic surfaces (6), electrical waves in retinal and cortical nerve tissue (7), waves in heart muscle (8), and cAMP (cyclic adenosine monophosphate) waves in starved population of Dictyostelium discoideum (D.d.) amobae $(9,10)$. One of the important questions concerning nonlinear waves is how they propagate in the presence of obstacles. Various aspects of this question, such as the influence of one or few large obstacles or a large number of small obstacles on wave propagation in excitable media, have been extensively studied (11-13).

Here, we report experimental and numerical results on the spatio-temporal dynamics of population of D.d. cells in the presence of non-excitable obstacles. This organism, naturally occurring in the forest soil, is an important model system for the study of chemotaxis, cell differentiation, and morphogenesis (14). Starvation of D.d. cells induces a developmental program in which cells align to form head-to-tail streams by signaling to each other with cAMP. Cells initiate the process by sending out pulsatile signals with a periodicity of several minutes, which propagate as waves. Over time, circular and

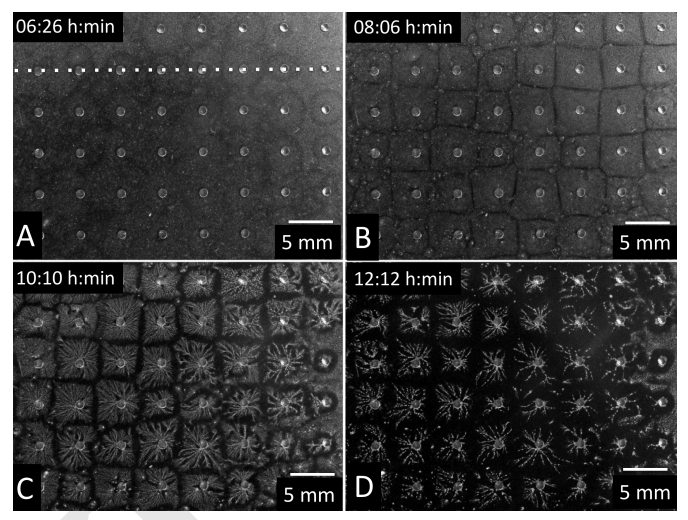

Fig. 1. Top view of D.d. cells on a macro-pillar array. A) Concentric waves initiate around the pillars and propagate outwards. (B-D) The amoebas respond chemotactically to the circular waves and stream towards the posts. This leads to the formation of regular domains around the pillars. Timestamps denote time since start of starvation.

spiral patterns form. Cells respond chemotactically to cAMP waves that guide cell movement towards the signaling centers and form multi-cellular centimeter-scale domains. The corresponding wave sources in each domain then act as aggregation centers, which eventually transform into millimeter long slugs and finally into fruiting bodies bearing spores for long-term survival and long-range dispersal (15). In their natural habitat, populations of starving cells are exposed to spatial hetero-

\section{Significance Statement}

Large-scale CAMP waves synchronize the population of starving Dictyostelium cells and enable them to aggregate and form a multi-cellular organism. In nature, Dictyostelium cells are exposed to external obstacles, which can significantly influence the wave generation process. Here, we confirm experimentally that spatial heterogeneities can shape the collective behavior of Dictyostelium cells only in the experiments where the excitability threshold of the cells is reduced; otherwise the obstacles are ignored. We used a reaction-diffusion model to identify the underlying mechanism for unexcitable obstacles to act as a wave source. These results are crucial to understand the signaling mechanism of Dictyostelium cells in the presence of spatial heterogeneities and expected to be generic to other excitable media governed by reaction-diffusion equations.

T.E. performed experiments, E.V.H. designed and performed numerical simulations, T.E., E.V.H A.B and A.G. analyzed data, A.B. and A.G. designed research, T.E., E.V.H., A.B., and A.G wrote the paper.

The authors declare no conflict of interest.

${ }^{1}$ T.E. and E.V.H. contributed equally to this work.

${ }^{2}$ To whom correspondence should be addressed. E-mail: azam.gholami@ds.mpg.de 
geneities that will profoundly influence the processes of wave generation, propagation, and aggregation. Here, we report experimental and numerical results on the spatio-temporal dynamics of population of D.d. cells in the presence of obstacles. We show that inert obstacles can act as aggregation centers if some accumulation of either cells or cAMP occurs around them. To prevent this, cell sensitivity to cAMP in wild-type cells must be low enough, in order to not aggregate around physical obstacles which would prevent further development. We expect our experiments to help understanding aggregation in complex environments, and more broadly, how self-organizing living systems regulate their parameters in order to survive according to their particular reaction-diffusion characteristics and environmental restrictions.

\section{Results}

Experimental Results. In nature, the obstacles encountered by D.d. are randomly distributed in $3 \mathrm{D}$, but as a first step towards understanding, we look at a simpler system of cells in a $2 \mathrm{D}$ geometry with a periodic arrangement of obstacles. Our quasi 2D geometry consists of a regular array of millimeter-sized pillars that control spatio-temporal dynamics of a population of uniformly distributed D.d. cells (see SI Appendix, Fig. S1). Unlike other excitable systems, initial experiments showed no interaction between obstacles and the appearing cAMP waves. We observed spirals pinned to the unexcitable obstacles when they, by chance, appeared close to one, but showed no systematic attraction or repulsion to them (see SI Appendix, Fig. S2 and movie 1).

Following the results of our numerical simulations (see next section) we lowered the excitation threshold of the cells by adding caffeine to the system. The resulting aggregation pattern is shown in Fig. 1. Under these conditions the spatial heterogeneities induce circular waves centered on the posts that trigger chemotactic cell movement towards the pillars. This leads to the formation of periodic domains that reflect the periodicity of the underlying macro-pillar array. Interestingly, we observed synchronized circular waves and regular domains only in the presence of caffeine (see Fig. S2 and movie 1 for an experiment in the absence of caffeine). Moreover, a similar phenomenon was observed in the experiments where we used holes instead of pillars as obstacles.

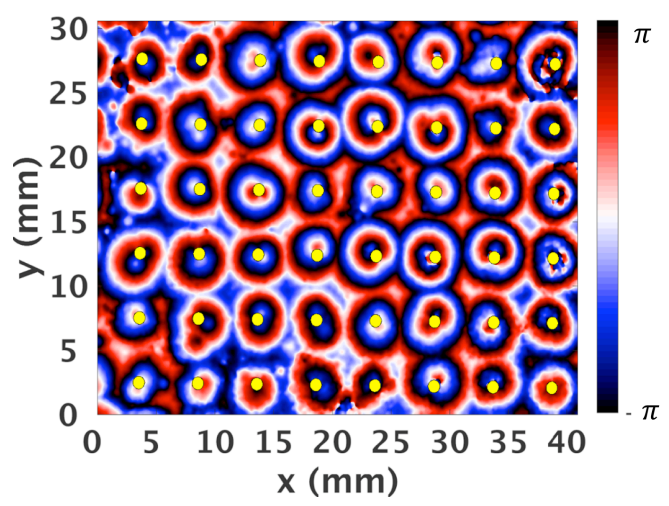

Fig. 2. Phase map of the observed spatio-temporal pattern showing the formation of circular waves around the pillars. Movie 5 shows the time evolution of the phase map.

In the absence of pillars, target patterns emerge at random locations on the PDMS substrate (see SI Appendix, Fig. S3

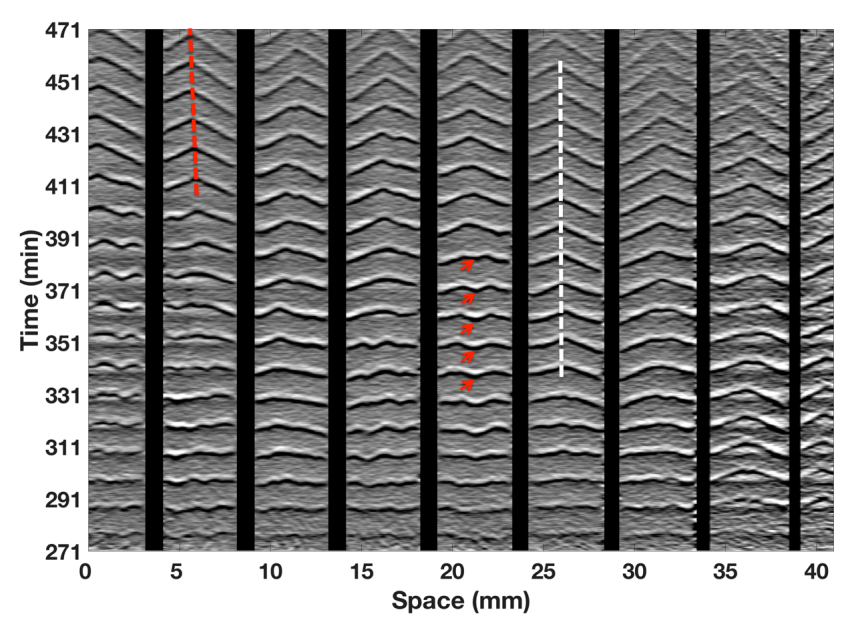

Fig. 3. Space-time plot of the experiment in Fig. 1. The light intensity from supplemental movie 4 is stacked up along the white dashed line shown in Fig. 1A. The black bars show the position of the pillars. The red arrows show a firing center other than the pillars that has a lower frequency and disappears with time. The white dashed line traces the annihilation points of two waves that initiate on the neighboring post and have a similar frequency but a phase shift. The red dashed line shows the slow drift of the annihilation point towards the post with smaller frequency (left pillar).

and movie 2). This is in contrast with patterns in the presence of macro-pillars (see movie 3), where waves originate at the posts and propagate outwards, as shown in Fig. 1. The four successive snapshots reveal circular waves centered around the pillars, cell streaming towards the posts, formation of regular domains, and cell aggregation, respectively. In our experiments, we observed that concentric waves develop almost synchronously around the pillars and since they have a slightly higher frequency, dominate over the other firing centers. Waves propagate outwards from the posts and trigger chemotactic movement of the cells towards the pillars. As a result, periodic domains form around the pillars.

The concentric waves around the pillars are well visible in the spatial phase map in Fig. 2 as well as in the processed movie 4 . In this video, the frames in movie 3 are subtracted every $1 \mathrm{~min}$ and band-passed filtered to reduce the spatial noise To calculate phase at each pixel, we first filter images in movie 3 (no image subtraction) and then use the Hilbert transform (see movie 5). The phase map shows that (i) the circular waves are slightly off-center from the pillars (ii) the territories that each circular wave propagates before annihilation have different sizes, and (iii) the frequency and phase of the circular waves can vary between the pillars.

Figure 3 shows the space-time plot along the white dashed line in Fig. 1A. For this kymograph, the processed images in movie 4 are used. First, we observe synchronized bulk oscillations (with a period around $10 \mathrm{~min}$ ). We attribute this to initial starvation of the cells in a shaking suspension for four hours that leads to cell synchronization. After about $60 \mathrm{~min}$, waves are initiated almost synchronously from the pillars and propagate outwards. They annihilate as they collide with each other. These annihilation areas define the boundaries of the regular domains. In an ideal experiment, where the circular waves have the same frequency and phase, the size of the quadratic domains is the same as the pillar spacing $(\sim 5 \mathrm{~mm})$. However, in general there is a frequency and phase difference between concentric waves originating from neighboring posts 


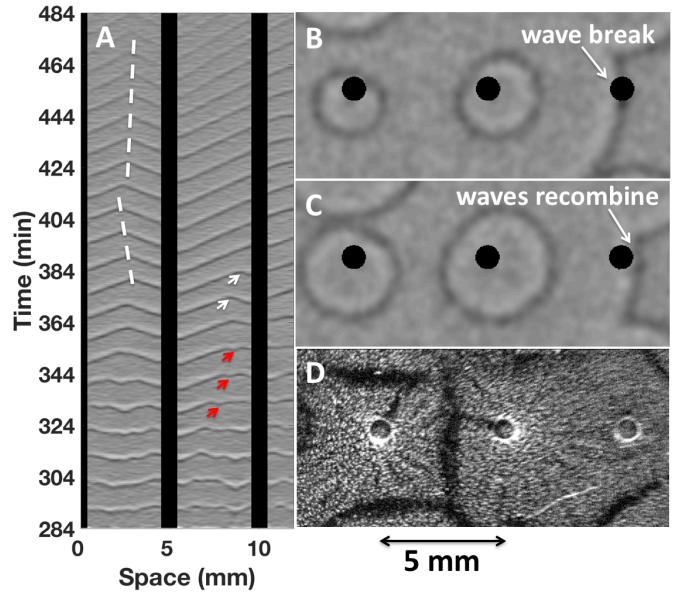

Fig. 4. A) Space-time plot in an experiment with pillars of $50 \mu \mathrm{m}$ high (SI Appendix movie 6). Only part of the kymograph with 3 pillars is shown. The red arrows point to a firing center other than the pillars which has a lower frequency and disappears with time. The white dashed lines show the movement of the annihilation points towards the pillars with lower wave frequency. The white arrows show the collision of the annihilation point with the right pillar. This pillar fails to emit its own circular cAMP wave and only breaks the wave front initiated from the neighboring pillar (B-D) As the wave front collides with the pillar on the right, it breaks and recombines again. As a result no boundary between two neighboring pillars forms.

consistent with the variety in target centers observed in other excitable systems (16). If two neighboring waves have almost the same frequency with a phase shift, due to this phase difference, the annihilation point of two neighboring circular waves is not located exactly at the middle of two pillars, but is rather located closer to the pillar with a phase delay. This is shown by the white dashed line in Fig. 3. Moreover, often in the experiments, there is also a small frequency difference between the waves initiating at the neighboring pillars. Consequently, the annihilation point of two neighboring concentric waves shifts towards the pillar with smaller frequency. This event is shown by the red dashed line in Fig. 3 and white dashed lines in Fig. 4A. The drift velocity of the annihilation point can be calculated to be $v_{w}\left(f_{2}-f_{1}\right) /\left(f_{2}+f_{1}\right)$, where $v_{w}$ is the wave propagation velocity and $f_{1}$ and $f_{2}$ are the wave frequencies at the two neighboring posts (SI Appendix, Fig. S4). In our experiments, $v_{w}$ is of the order of $0.4 \mathrm{~mm} / \mathrm{min}$ and the frequencies $f_{1}$ and $f_{2}$ are roughly $1 / 10 \mathrm{~min}^{-1}$ and $1 / 9 \mathrm{~min}^{-1}$, which gives $v_{\text {drift }}$ to be of the order of $0.02 \mathrm{~mm} / \mathrm{min}$. If the frequency difference persists, eventually the wave front collides with the pillar and breaks as is shown by white arrows in Figs. 4A, B. They recombine after passing through the obstacle and propagate further (Fig. $4 \mathrm{C}$ ). In this case, the obstacle only breaks the propagating wave front and there is no boundary formed between the middle and the right pillar in Fig. 4D.

In Fig. 5A, we calculated the gradient vectors of the phase map around two neighboring posts. The vector field changes direction where the concentric waves meet and annihilate each other. Thus, the Laplacian of the phase map $\phi$, defined as $\partial^{2} \phi(x, y) / \partial x^{2}+\partial^{2} \phi(x, y) / \partial y^{2}$, takes on extreme values at the collision regions of two neighboring emitted waves and defines the boundaries of the domains (see Fig. 5B).

The phenomenon of initiation of synchronized circular waves around the pillars and formation of regular domains

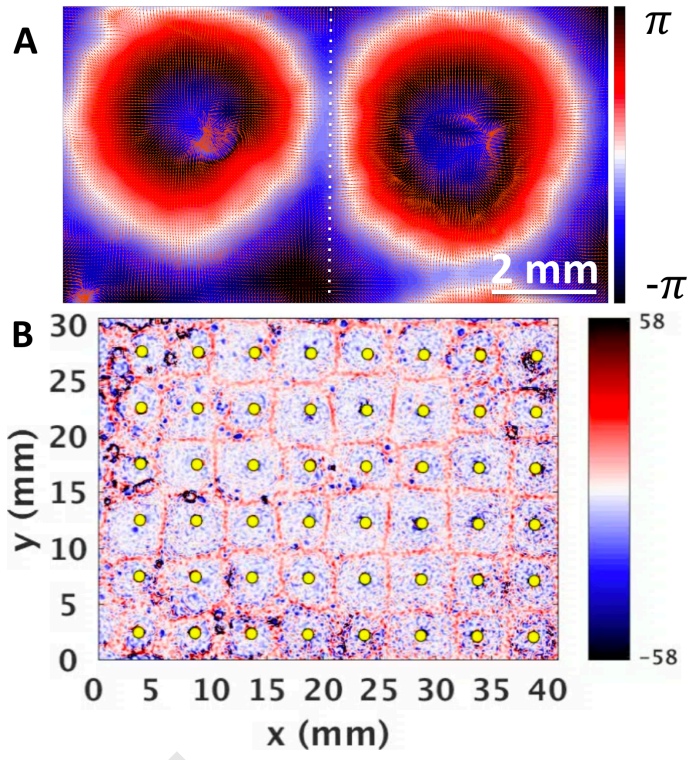

Fig. 5. A) Gradient vectors of the phase map around two neighboring posts. The white dashed line shows the location where two emitted waves collide and the vector field changes direction. The vectors are scaled 50 times larger than the original values for better visibility. B) Laplacian of the phase map in unit of $\mathrm{mm}^{-2}$. The gradient vector field switches direction at the boundary of the domains, thus Laplacian has higher values at these boundaries.

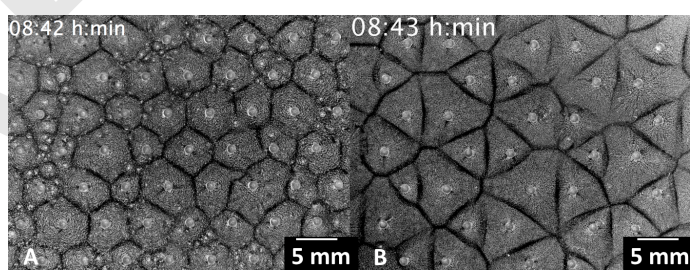

Fig. 6. Hexagonal and triangular domains formed around the pillars with A) triangular and $\mathrm{B}$ ) hexagonal arrangement, respectively (SI Appendix, movies 7 and 8).

were robust with respect to the arrangement of the pillars. We repeated our experiments with the same pillar size and spacing, but triangular and hexagonal arrangements of the posts, and observed hexagonal and triangular domains, respectively (Fig. 6 and SI Appendix, movies 7, 8).

Numerical Simulations. We performed numerical simulations of the model proposed by Martiel and Goldbeter (MG) for the creation and relay of cAMP in D.d. (17, 18). Many approaches have been used to create spirals and target centers in this model, most of which define the position of the localized structures through perturbations or diversity of developmental stage among the cells $(19,20)$. We instead used a dynamical approach where centers appear naturally in areas of higher local density (see Materials and Methods and SI Appendix movie 9). Here, the cell locations are discretized inside grids, thus a grid containing a cell is an occupied one and can produce and relay cAMP, while the empty grids without cells can only degrade the signal via external phosphodisterase. This mixture of occupied and unoccupied areas on the system breaks the system homogeneity and allows clusters of local higher cell density to become target centers. The lower density areas are still capable of sustaining waves, thus the waves generated 
by these clusters get relayed by the rest of the system. We measured the dispersion relation of such waves and showed that they have the behavior of trigger waves (21)(see Fig. 7B). These dispersion relations at different cell densities also showed that the wave velocity increases with cell density, which has been reported to be necessary to produce aggregation streams (22).

We observed in our simulations that a no flux boundary condition at the obstacles is not sufficient to produce centers. Similarly, when this boundary condition is applied, waves only break after colliding with the obstacles and recombine again (SI Appendix, Fig. S5 and movie 10). This was in agreement with our initial experiments without caffeine. Another boundary condition that has been shown to produce traveling waves in numerical simulations is the Dirichlet boundary condition, where the boundary is held to a fixed value. In our system, a fixed value of cAMP at the obstacles creates wave trains emitted from the obstacles if the fixed value is larger than a threshold, as it is shown in Fig. 8 and the supplemental movie 11. This minimum amount of cAMP needed for the obstacles to act as a wave source depends on the system parameters.
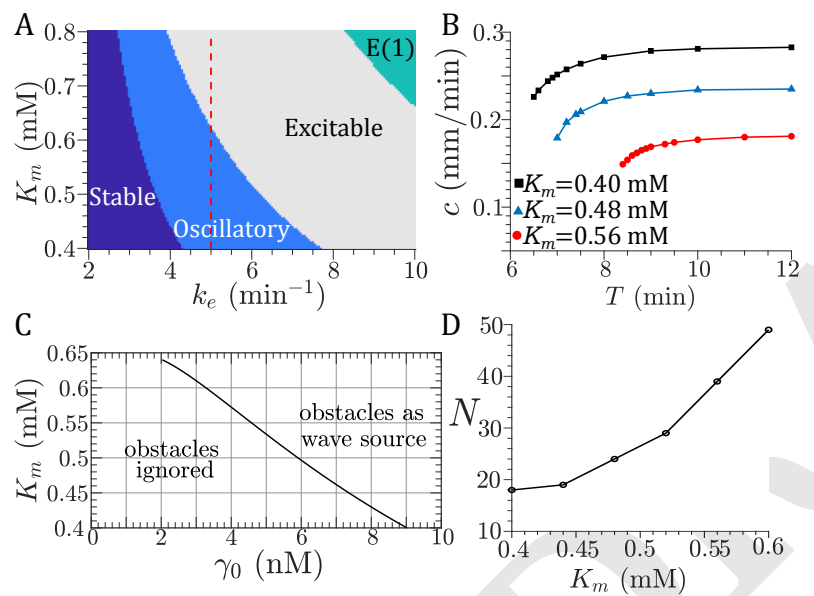

Fig. 7. A) Phase diagram of the MG model used for simulating the experimental setup. On the area marked as Stable, the system has one solution, which is stable. In the Oscillatory region, the system shows one unstable steady state surrounded by a limit circle. In the Excitable regime, the system has 3 steady states, two unstable ones and a stable one, which is excitable. In the regime marked as $E(1)$ the system shows one steady state, which is excitable. Stability calculated through linear analysis, and excitability through no-space simulations. Red dashed line shows the path that $K_{m}$ is changed in parts B, C and D at fixed value of $k_{e}=5.0 \mathrm{~min}^{-1}$. Note that higher $K_{m}$ values correspond to higher caffeine concentration. B) Dispersion relations of the supported wave-trains for $40 \%$ surface coverage, where $T$ is wave period and $c$ wave velocity. Waves with periods below those shown, do not get relayed by the system. C) Effect of a boundary condition $\gamma_{0}$ : for higher values of $K_{m}$ the minimum amount of $\gamma_{0}$ required to produce a target center decreases. D) Minimum number $N$ of consecutive cells (in 1-D) needed to produce a cluster with self-sustained oscillations.

The minimum accumulation of cAMP or cells necessary for an unexcitable obstacle to become a center is high enough so that the cells do not aggregate around the obstacles naturally. In order to obtain aggregation domains around the obstacles we varied different parameters which can be controlled in our experiments. To achieve a lower excitation threshold we varied the parameter $K_{m}$ which is the Michaelis constant of the reaction in which ATP produces intracellular cAMP. Increasing this parameter reduces the affinity between ATP and the enzyme adenylate cyclase (ACA), thus reducing the production rate of intracellular cAMP and accounting for the effects of adding caffeine to the experimental system. We performed linear stability analysis of the MG model with modifications in $K_{m}$ and characterized its different regimes, which are shown in Fig. 7A, the other parameter we varied is the degradation rate of external phosphodiesterase $k_{e}$. We chose the parameters such that the system is in the oscillatory regime, i.e. a stable limit cycle exists, and the cell coverage (ratio of occupied grids to total number of grids) is high enough for the waves to get relayed. At higher values of $K_{m}$, which is equivalent to higher concentration of caffeine in our experiments, the number of firing centers in the system is decreased (SI Appendix, Fig. S6 and movie 12). The reason is that the minimum cluster size (measured as consecutive cells in a 1D setting) required to produce a self-sustained oscillatory center is increased (see Fig. 7D). Increasing the amount of caffeine (higher $K_{m}$ values) also decreased the wave velocity and increased the minimum wave period the system can sustain. Different dispersion relations showing this effect are depicted in Fig. 7B. We studied the boundary effect in this system and found that at higher caffeine concentration the trigger waves require a smaller amount of cAMP to be triggered, thus the obstacles act as a wave source at smaller values of the fixed boundary $\gamma_{0}$ (see Fig. $7 \mathrm{C}$ ).

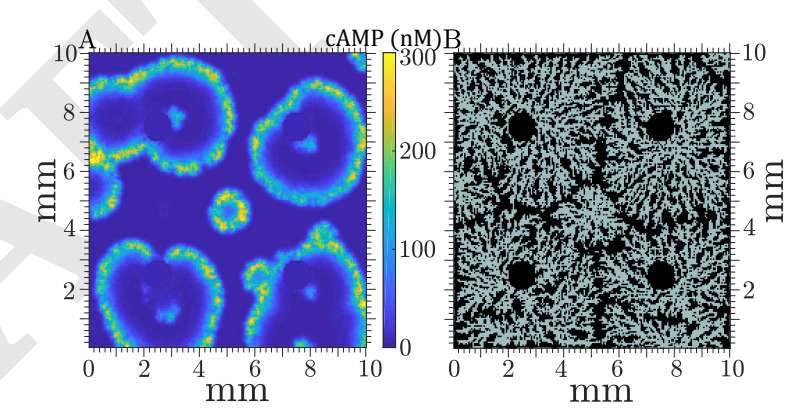

Fig. 8. Numerical simulations of CAMP waves with four pillars as obstacles with fixed boundary condition $\gamma_{0}=10 \mathrm{nM}, K_{m}=0.508 \mathrm{mM}, k_{e}=5.0 \mathrm{~min}^{-1}, 50 \%$ of cell surface coverage. A) Concentric waves of cAMP coming out of the pillars. B) Cell distribution after $t=100 \mathrm{~min}$, showing Voronoi domains around the pillars. Grey squares show grid points containing a cell, black squares show the empty ones.

Another mechanism for wave creation observed in our simulations was a higher local cell density around the pillars. This can be achieved either by inhomogeneous initial cell distribution or by adhesion of the cells to the pillars after colliding due to random movement. These locally high density cell clusters trigger the formation of wave centers and act as aggregation centers, as shown in SI Appendix, Fig. S7 and movie 13. Notably, we also performed numerical simulations with hexagonal and triangular arrangement of the pillars (Dirichlet boundary condition) and observed triangular and hexagonal patterns, respectively (see SI Appendix, Figs. S8, S9 and movies 14, 15).

Finally, it is experimentally suggested that the effect of caffeine on ACA inhibition is possibly mediated by different targets, one of which inhibits the activation of G-protein G2, that is part of the signal transduction cascade that normally activates ACA (23). To investigate the effect of having less activated ACA, we also performed simulations by modifying the parameter $\epsilon$ which controls the ratio of the active to inactive forms of ACA. These simulations showed similar results to those obtained by modifying $K_{m}$. Therefore, regardless of the particular way in which caffeine affects ACA, our numerical 
results provide explanation for the observed behavior (see SI Appendix, Figs. S10 and S11 and movie 16).

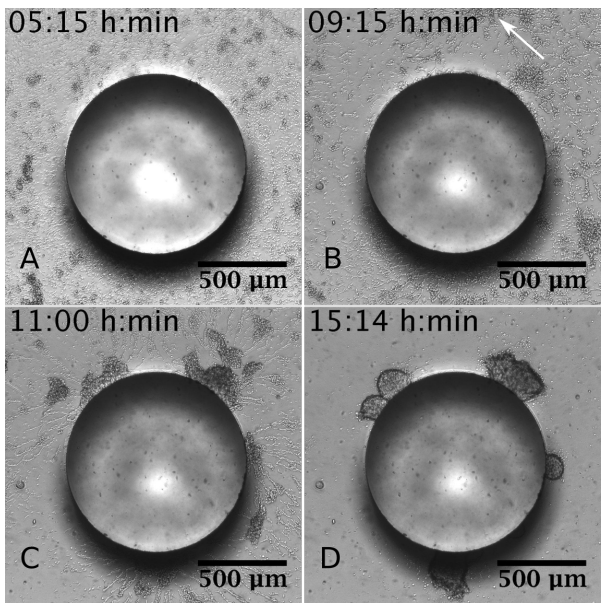

Fig. 9. A closer look at the pillars with a bright field microscope. A) Initial distribution of the cells show small clumps. B-D) Cells chemotactically move towards the center of the concentric waves that are sometimes slightly off-center from the pillars. During the streaming process, the cells join small clusters to make larger ones and eventually large clusters aggregate on the post (see SI Appendix, movie 17).

Bright Field Microscopy. Next, we used bright field microscopy to look closely at the wave propagation and cell streaming in the vicinity of the posts (SI Appendix, movie 17). A higher cell density around the pillars after plating the cells could explain the higher wave frequency of the waves initiating from the posts. Our extensive bright field observations did not confirm a significant cell accumulation around the pillars (SI Appendix, Fig. S12). However, based on our numerical simulations, in the presence of caffeine even a tiny cAMP accumulation in the vicinity of the obstacles is enough to trigger formation of concentric waves around the posts. Possible cell attachment to the side walls of the pillars which is considered in simulations with a small constant value of cAMP on the obstacles, is a plausible mechanism for the formation of circular waves around the posts. Moreover, we emphasize that initial starvation of the cells for 4 hours in shaken suspension leads to increased cell-cell adhesion, and very small cell clusters are visible in Fig. 9A. As we mentioned before, concentric waves around the pillars are not always all centered at the pillars, but are slightly off-center. Therefore, the cells first stream towards a point in the vicinity of the pillar (arrow in Fig. 9B and SI Appendix, movie 17) and then aggregate at the pillar itself.

\section{Discussion}

The results presented here show that external obstacles can significantly influence the generation of waves in starving populations of D.d. cells when the excitability threshold has been lowered. We observed circular waves that initiate almost synchronously at the pillars and propagate outwards. Chemotactically competent cells detect the cAMP gradient and crawl towards the posts, forming a periodic array of domains. This phenomenon is also observed for triangular and hexagonal arrangement of the pillars leading to the formation of hexagonal and triangular domains, respectively. Most of our experiments were performed with pillars of $1 \mathrm{~mm}$ diameter, but the phenomenon showed to be fairly robust to different types of obstacles, since we observed a similar phenomenon with pillars of $1.5 \mathrm{~mm}$ diameter (SI Appendix, movie 18), shallow holes $(100 \mu \mathrm{m})$ in PDMS (SI Appendix, movie 19), smaller center-to-center spacing of the pillars $(3.75 \mathrm{~mm}$ instead of $5 \mathrm{~mm}$ ), and even with a PDMS wall (SI Appendix, movie $20)$. The phenomenon is also robust with respect to the pillar height since we observed regular domains with pillar's height down to 50 micron (see Fig. 4 and SI Appendix, movie 6). The concentric waves emitted from pillars have a slightly higher frequency than the other firing centers, thereby dominating the system dynamics. Cells attached to the side walls of the PDMS can trigger higher frequency waves. In our simulations, we included this effect by assuming either a fixed value of cAMP around the posts or slightly higher cell accumulation in the vicinity of pillars. Future experiments using fluorescent indicators for extracellular cAMP, will be valuable to visualize any possible cAMP accumulation around the pillars $(24,25)$. Another scenario which requires extra bio-chemical analysis, is the possibility of accumulation or depletion of any chemical (such as phosphodiestrase) in the vicinity of the pillars. Our numerical simulations show that in the case of PDE depletion, pillars can act as wave source in the system that might drive oscillations in the vicinity of the obstacles (26-28) (SI Appendix, movie 21). In the opposite case of PDE accumulation, pillars are "ignored" and only break the colliding wave fronts (SI Appendix, movie 22). However, we should mention that, to prevent adsorption of chemicals to the PDMS substrate, we repeated our experiments with BSA (Bovine Serum Albumin) treated substrates and still observed concentric waves and periodic domains around the pillars. Finally, although PDMS is useful because of its bio-compatibility, deformability and permeability to gas, it has certain properties which might complicate our results. To rule this out we used pillars made of PMMA and again observed the regular streaming domains.

In our system, we observed periodic domains within a range of caffeine concentration between 1-5 mM. Caffeine, which is a highly specific inhibitor of cAMP relay (29-31), reduces both the cAMP production rate and wave frequency in a dosedependent manner (SI Appendix, Figs. S13 and S14). We did not observe ordered aggregation territories without caffeine (SI Appendix, Fig. S2 and movie 1). Our experiments and earlier experiments by C. Weijer et.al. (32) with caffeine have shown that the aggregation territories are much larger in the presence of the caffeine compared to the controlled experiments (SI Appendix, Fig. S15 and movie 23). This means that the number of firing centers is decreased, which is also consistent with our numerical simulations in the presence of caffeine with higher values of $K_{m}$ (SI Appendix, Fig. S6 and movie 12). We believe that in our experiments, a lower number of firing centers is crucial for circular waves emitted from pillars to successfully take over and dominate the system dynamics. We also observed that in the presence of obstacles, caffeine drastically diminished the number of spirals appearing in the system. Since spirals have a higher frequency than target patterns, they take over and dominate the dynamics once they appear. In other excitable systems, such as the BZ reaction it has been shown that spirals can be pinned to obstacles with lower excitability (13). In that reaction-diffusion system it has also been shown numerically that the spiral tip interacts with the (no flux) obstacle boundary through attraction and 
repulsion (33). We observe neither attraction nor repulsion of the spiral cores to the obstacles in both the experimental setup and the numerical simulations. We believe this crucial difference is given by the lack of meandering of the spiral tip in D.d. Additionally, the lack of attraction to obstacles makes sense from a survival point of view, since aggregating around an obstacle would complicate the creation of a mound and the consequent phases of the life cycle. Interestingly, it has been shown that in the presence of caffeine spiral core size increases in a dose dependent manner (31). In those occasions when a spiral did appear next to a pillar, it got pinned to the pillar and remained rotating around it (SI Appendix, Fig. S16 and movie 24).

Comparing our numerical simulations with modified $K_{m}$ to the unmodified parameters, the system showed fewer centers, due to higher minimum cluster size necessary to produce pacemakers; it also showed smaller frequency, in agreement with experimental measurements (SI Appendix, Figs. S13, S14). The minimum amount of cAMP necessary to produce cell activation was diminished, allowing for easier wave relay. This is consistent with experimental results in (29) showing that caffeine indeed increases apparent chemotactic sensitivity of the cells. Thus, in the presence of caffeine, the transition boundary between "source" and "ignored" obstacles occurs at lower values of cAMP accumulation around the obstacles. For this reason, the presence of caffeine is necessary in our experiments to trigger formation of concentric waves around the pillars. Since in nature an inhomogeneous distribution of cells is expected, we believe that cells need a tuned sensitivity capable of reacting to cAMP signaling but that prevents aggregation around inconvenient places which can complicate further development.

\section{Materials and Methods}

Experimental Methods. The D.d. cells (strain AX2-214) were grown at $22^{\circ} \mathrm{C}$ in $\mathrm{HL} 5$ medium, harvested in the exponential growth phase, and starved for four hours in $10 \mathrm{~mL}$ phosphate buffer supplemented with $2 \mathrm{mM}$ caffeine in a shaking suspension. After 4 hours of starvation, they were centrifuged and diluted to a density of $2 \times 10^{6} \mathrm{cells} / \mathrm{ml}$ in fresh phosphate buffer containing $2 \mathrm{mM}$ caffeine. Next, $20 \mathrm{~mL}$ cell solution $\left(\sim 0.9 \times 10^{6}\right.$ cells $/ \mathrm{cm}^{2} \sim 0.9$ mono-layer $)$ was transfered to a modified Petri dish with a plasma-treated polydimethylsiloxane (PDMS) substrate (34). The PDMS has a periodic array of macro-pillars characterized by pillar dimensions and spacing (see SI Appendix, Fig. S1). If not stated otherwise, pillars of $1 \mathrm{~mm}$ diameter and height of $3 \mathrm{~mm}$ are arranged on square, triangular or hexagonal lattices with lattice size of $5 \mathrm{~mm}$. cAMP wave patterns are indirectly visualized by dark-field microscopy (10).

Numerical Methods. The reaction-diffusion equations used for modeling this system are

$$
\begin{aligned}
k_{1}^{-1} \partial_{t} \rho_{i} & =-f_{1}\left(\gamma\left(x_{i}, y_{i}\right)\right) \rho_{i}+f_{2}\left(\gamma\left(x_{i}, y_{i}\right)\right)\left(1-\rho_{i}\right), \\
\partial_{t} \beta_{i} & =s \Phi\left(\rho_{i}, \gamma\left(x_{i}, y_{i}\right)\right)-\left(k_{i}+k_{t}\right) \beta_{i}, \\
\partial_{t} \gamma & =D \nabla^{2} \gamma-k_{e} \gamma+\sum_{i}^{N} H(i, x, y) k_{t} \beta_{i} / h,
\end{aligned}
$$

with $f_{1}(\gamma)=\frac{1+\kappa \gamma}{1+\gamma}, f_{2}(\gamma)=\frac{\mathcal{L}_{1}+\kappa \mathcal{L}_{2} c \gamma}{1+c \gamma}, \Phi(\rho, \gamma)=\frac{\lambda_{1}+Y^{2}}{\lambda_{2}+Y^{2}}$, $Y(\gamma, \rho)=\frac{\rho \gamma}{1+\gamma}$ and $s=q \sigma \alpha /(1+\alpha)$, where $\gamma(x, y)$ and $\beta_{i}$ are the amount of extracellular and intracellular cAMP respectively. $\rho_{i}$ corresponds to the percentage of active cAMP receptors on the cell surface, $k_{e}$ corresponds to the extracellular phosphodiesterase and $s$ controls the amount of cAMP produced inside the cells. See SI Appendix for the used parameters and the motility rules.

ACKNOWLEDGMENTS. We are grateful to E. Bodenschatz, E Frey, V. Zykov, O. Steinbock, F. M.-Rafiee, L. Turco, and I. Guido for fruitful discussions. T. E. acknowledges Deutsche Forschungsgemeinschaft (DFG), project Nr. GH184/1-1. E.V.H. thanks the Deutsche Akademische Austauschdienst (DAAD), Research Grants - Doctoral Programs in Germany. A.G. acknowledges the MaxSynBio Consortium which is jointly funded by the Federal Ministry of Education and Research of Germany and the Max Planck Society.

1. Holden AV, Markus M, Othmer H (2013) Nonlinear wave processes in excitable media (Springer) Vol. 244.

2. Grindrod P (1991) Patterns and waves: The theory and applications of reaction diffusion equations. clarendon.

3. Meron E (1992) Pattern formation in excitable media. Physics reports 218(1):1-66.

4. Zykov VS (1987) Simulation of wave processes in excitable media. (Manchester Univ Press, Manchester, UK).

5. Zaikin A, Zhabotinsky A (1970) Concentration wave propagation in two-dimensional liquidphase self-oscillating system. Nature 225(5232):535-537.

6. Imbihl R, Ertl G (1995) Oscillatory kinetics in heterogeneous catalysis. Chemical Reviews 95(3):697-733.

7. Gorelova N, Bureš J (1983) Spiral waves of spreading depression in the isolated chicken retina. Developmental Neurobiology 14(5):353-363.

8. Davidenko JM, Pertsov AV, Salomonsz R, Baxter W, Jalife J (1992) Stationary and drifting spiral waves of excitation in isolated cardiac muscle. Nature 355(6358):349-351.

9. Gerisch Gt (1965) Stadienspezifische aggregationsmuster beidictyostelium discoideum Development Genes and Evolution 156(2):127-144.

10. Devreotes PN, Potel MJ, MacKay SA (1983) Quantitative analysis of cyclic amp waves mediating aggregation in dictyostelium discoideum. Developmental biology 96(2):405-415.

11. Panfilov AV, Keener JP (1993) Effects of high frequency stimulation on cardiac tissue with an inexcitable obstacle. Journal of theoretical biology 163(4):439-448.

12. Bär M, Meron E, Utzny C (2002) Pattern formation on anisotropic and heterogeneous catalytic surfaces. Chaos: An Interdisciplinary Journal of Nonlinear Science 12(1):204-214.

3. Steinbock O, Müller S (1992) Chemical spiral rotation is controlled by light-induced artificial cores. Physica A: Statistical Mechanics and its Applications 188(1-3):61-67.

14. Chisholm RL, Firtel RA (2004) Insights into morphogenesis from a simple developmental system. Nature reviews. Molecular cell biology 5(7):531.

15. Saran S, et al. (2002) camp signaling in dictyostelium. Journal of Muscle Research \& Cell Motility 23(7-8):793-802.

16. Kuramoto $Y(2012)$ Chemical oscillations, waves, and turbulence. (Springer Science \& Business Media) Vol. 19

17. Martiel JL, Goldbeter A (1987) A model based on receptor desensitization for cyclic AMP signaling in Dictyostelium cells. Biophysical Journal 52(5):807.

18. Tyson JJ, Alexander KA, Manoranjan V, Murray J (1989) Spiral waves of cyclic AMP in a model of slime mold aggregation. Physica D: Nonlinear Phenomena 34(1-2):193-207.

19. Lauzeral J, Halloy J, Goldbeter A (1997) Desynchronization of cells on the developmental path triggers the formation of spiral waves of CAMP during Dictyostelium aggregation Proceedings of the National Academy of Sciences 94(17):9153-9158.

20. Geberth D, Hütt MT (2009) Predicting the distribution of spiral waves from cell properties in a developmental-path model of dictyostelium pattern formation. PLoS computational biology 5(7):e1000422.

21. Aliev RR, Biktashev VN (1994) Dynamics of the oscillation phase distribution in the bz reaction. The Journal of Physical Chemistry 98(38):9676-9681.

22. Van Oss C, Panfilov AV, Hogeweg P, Siegert F, Weijer CJ (1996) Spatial pattern formation during aggregation of the slime moulddictyostelium discoideum. Journal of theoretical biology 181(3):203-213.

23. Alvarez-Curto E, Weening KE, Schaap P (2007) Pharmacological profiling of the dictyostelium adenylate cyclases aca, acb and acg. Biochemical Journal 401(1):309-316.

24. Ohta Y, Furuta T, Nagai T, Horikawa K (2018) Red fluorescent camp indicator with increased affinity and expanded dynamic range. Scientific reports $8(1): 1866$

25. Gregor T, Fujimoto K, Masaki N, Sawai S (2010) The onset of collective behavior in social amoebae. Science 328(5981):1021-1025.

26. Stich M, Mikhailov AS (2002) Complex pacemakers and wave sinks in heterogeneous oscillatory chemical systems. Zeitschrift für Physikalische Chemie 216(4):521.

27. Gholami A, Steinbock O, Zykov V, Bodenschatz E (2015) Flow-driven instabilities during pattern formation of Dictyostelium discoideum. New Journal of Physics 17(6):063007.

28. Vidal-Henriquez E, Zykov V, Bodenschatz E, Gholami A (2017) Convective instability and boundary driven oscillations in a reaction-diffusion-advection model. Chaos 27(10):103110.

29. Brenner M, Thoms SD (1984) Caffeine blocks activation of cyclic amp synthesis in dictyostelium discoideum. Developmental biology 101(1):136-146.

30. Jaiswal P, Soldati T, Thewes S, Baskar R (2012) Regulation of aggregate size and pattern by adenosine and caffeine in cellular slime molds. BMC developmental biology 12(1):5.

31. Steinboek O, Müller SC (1995) Spatial attractors in aggregation patterns of dictyostelium discoideum. Zeitschrift für Naturforschung C 50(3-4):275-281.

32. Siegert F, Weijer C (1989) Digital image processing of optical density wave propagation in dictyostelium discoideum and analysis of the effects of caffeine and ammonia. Journal of Cell Science 93(2):325-335.

33. Munuzuri AP, Pérez-Muñuzuri V, Pérez-Villar V (1998) Attraction and repulsion of spiral waves by localized inhomogeneities in excitable media. Physical Review E 58(3):R2689. 34. Whitesides GM, Ostuni E, Takayama S, Jiang X, Ingber DE (2001) Soft lithography in biology and biochemistry. Annual review of biomedical engineering 3(1):335-373. 


\section{Supplemental Information: Spatial heterogeneities shape collective behavior of signaling amoeboid cells}

Torsten Eckstein, Estefania Vidal-Henriquez, Abert J. Bae, and Azam Gholami

Max Planck Institute for Dynamics and Self-Organization, Am Fassberg 17, 37077 Göttingen, Germany

\section{Experimental set up}

Figure S1 shows the PDMS mold with quadratic arrangement of the pillars, which is used in our experiments.

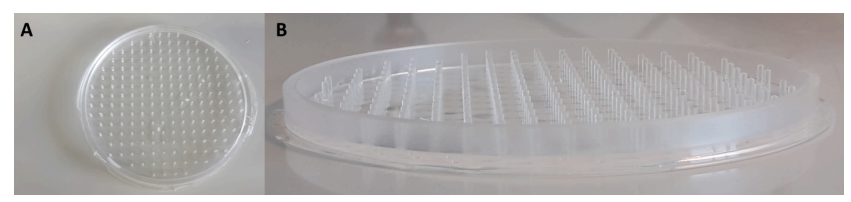

Fig.S 1. (A) Top view and (B) side view of the PDMS block with a quadratic arrangement of the pillars that fits to a normal $100 \mathrm{~mm}$ plastic Petri dish. The pillars are $1 \mathrm{~mm}$ thick, $3 \mathrm{~mm}$ high and are spaced $5 \mathrm{~mm}$ away from each other.

\section{Experiments with and without caffeine in the presence and absence of pillars}

To repeat the exact experimental conditions similar to our pillar experiments, we performed an experiment in the presence of $2 \mathrm{mM}$ caffeine and 4 hours initial starvation but no pillars. The result of this experiment with PDMS substrate is shown in Fig. S3. It is important to emphasize that the initial starvation time of the cells plays an important role in the pattern formation of D.d. cells, as investigated in detail by Prabhakara et.al. (1). Figure S15 shows two experiments in the presence and absence of caffeine both without pillars but PDMS substrate. For these two experiments, the cells are not initially starved in a shaking suspension. Note that in the presence of caffeine, the number of aggregation centers is reduced.

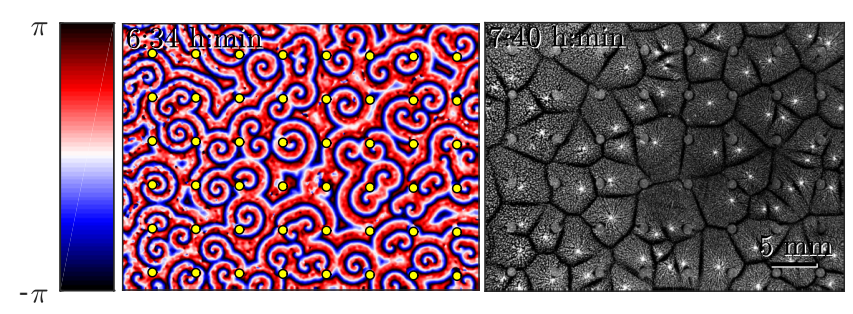

Fig.S 2. Phase map of cAMP waves and aggregation domains in an experiment with pillars but in the absence of caffeine. Cells are initially 4 hours starved in a shaking suspension (see also supplemental movie 1).

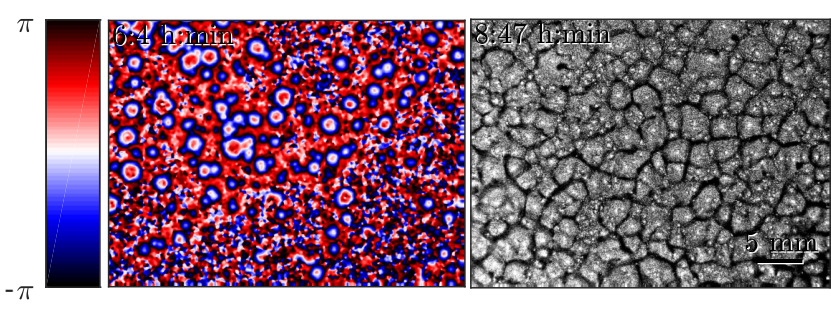

Fig.S 3. Phase map of cAMP waves and aggregation domains in an experiment without pillars in the presence of $2 \mathrm{mM}$ caffeine. Note that target patterns are dominated. To keep the conditions similar to the pillar experiments, the cells are initially 4 hours starved in a shaking suspension and the substrate is PDMS (see also movie 2).

\section{Numerical simulations of the MG model}

The reaction-diffusion equations used for modeling this system are

$$
\begin{aligned}
k_{1}^{-1} \partial_{t} \rho_{i} & =-f_{1}\left(\gamma\left(x_{i}, y_{i}\right)\right) \rho_{i}+f_{2}\left(\gamma\left(x_{i}, y_{i}\right)\right)\left(1-\rho_{i}\right), \\
\partial_{t} \beta_{i} & =s \Phi\left(\rho_{i}, \gamma\left(x_{i}, y_{i}\right)\right)-\left(k_{i}+k_{t}\right) \beta_{i}, \\
\partial_{t} \gamma & =D \nabla^{2} \gamma-k_{e} \gamma+\sum_{i}^{N} H(i, x, y) k_{t} \beta_{i} / h,
\end{aligned}
$$

with $f_{1}(\gamma)=\frac{1+\kappa \gamma}{1+\gamma}, f_{2}(\gamma)=\frac{\mathcal{L}_{1}+\kappa \mathcal{L}_{2} c \gamma}{1+c \gamma}, \Phi(\rho, \gamma)=$ $\frac{\lambda_{1}+Y^{2}}{\lambda_{2}+Y^{2}}, Y(\gamma, \rho)=\frac{\rho \gamma}{1+\gamma}$ and $s=q \sigma \alpha /(1+\alpha)$, where $\gamma(x, y)$ and $\beta_{i}$ are the amount of extracellular and intracellular cAMP respectively. $\rho_{i}$ corresponds to the percentage of active cAMP receptors on the cell surface and acts effectively as the slow variable that gives the system its excitable capabilities. $k_{e}$ corresponds to the extracellular phosphodiesterase and $s$ controls the amount of cAMP produced inside the cells. $H(i, x, y)$ is an index variable with values 1 if the $i-t h$ cells is located in $(x, y)$ and 0 if it is not. This produces that on the grid points containing amoebas the cAMP is transported from the intra- to the extracellular media, while on the empty cell spaces the wave is degraded by the unbounded phosphodiesterase. The systems was simulated using discrete differences, a 5 point laplacian, and a time adaptive Runge-Kutta scheme. The used parameters were $\sigma=0.55 \mathrm{~min}^{-1}, k_{1}=0.09 \mathrm{~min}^{-1}$, $\kappa=18.5, \mathcal{L}_{1}=10, \mathcal{L}_{2}=0.005, c=10, q=4000, \alpha=1.2 / K_{m}$, $\lambda_{1}=10^{-4} / \epsilon, \lambda_{2}=0.2575 / \epsilon, k_{i}=1.7 \mathrm{~min}^{-1}, k_{t}=0.9 \mathrm{~min}^{-1}$, $D=0.024 \mathrm{~mm}^{2} / \mathrm{min}$, and $h=5$. For simulating the effects of caffeine either $K_{m}=0.4-0.6 \mathrm{mM}$ and $\epsilon=1.0$ or $K_{m}=0.4$ $\mathrm{mM}$ and $\epsilon=0.6-1.0$ are used. Note that higher $K_{m}$ values 
correspond to higher concentrations of caffeine, while higher values of $\epsilon$ is a measure of lower caffeine concentrations (see movie 16 for a simulation of different values of $\epsilon$ ). With these parameters the system is in an oscillatory state, meaning that a limit cycle exists. If a cluster of cells of big enough size exists, it acts as a pacemaker producing trigger (chemical) waves that are relayed by the system, as long as a minimum percentage (measured as surface coverage) of cells exists. Emulating the experimental observations, after some simulation time, when the waves have been established, we allowed the cells to be chemotactically competent. The movement rules were as follows. If a cell detects a cAMP gradient bigger than a threshold $\left(\nabla \gamma>g_{t h}\right)$ and it is in the excitable state $\left(\rho>\rho_{t h}\right)$, it moves against the gradient with a velocity $v_{c}$ as long as the two previous conditions continue to be fulfilled. If the new position falls in a different grid space, the movement occurs only if the new grid point does not already contain a cell. The parameters used were $g_{t h}=25.98 \mathrm{nM} / \mathrm{mm}, \rho_{t h}=0.6, v_{c}=20$ $\mu \mathrm{m} / \mathrm{min}$, and we allowed cell movement after $t=50 \mathrm{~min}$.

\section{Drift velocity of the annihilation point}

We draw vertical lines starting at the annihilation point $B$ until the intersection with the wave $D$, and similarly we draw a line up from the later annihilation point $C$ up until the intersection $A$. The periods of the waves initiating from left and right pillars are $T_{1}$ and $T_{2}$, respectively (see Fig. S4). Since $\overline{A C}$ and $\overline{B D}$ are parallel, the quadrilateral $A B C D$ is a scalene trapezoid with bases $\overline{A C}=T_{1}$ and $\overline{B D}=T_{2}$. The basal angles correspond with the wave propagation velocities such that $\angle B A C=\alpha$ and $\angle A C D=\beta$ with $\tan \alpha=v_{1}, \tan \beta=v_{2}$.

Using the law of sines we calculate the other two sides of the trapezoid and arrive at

$$
\overline{A B}=\left(T_{1}-T_{2}\right) \frac{\sin \beta}{\sin (\alpha+\beta)}, \quad \overline{C D}=\left(T_{1}-T_{2}\right) \frac{\sin \alpha}{\sin (\alpha+\beta)} .
$$

We now look at the angle $\angle C B D=\gamma$ such that $\tan \gamma=v_{d}$ is the drift velocity of the annihilation point. Applying law of sines to the triangle $\triangle C B D$ we get

$$
\frac{\sin (\angle B C D)}{\overline{B D}}=\frac{\sin \gamma}{\overline{C D}}
$$

Substituting with the values already calculated we get

$$
\frac{\sin (\beta-\gamma)}{T_{2}}=\frac{\sin \gamma}{\left(T_{1}-T_{2}\right)} \cdot \frac{\sin (\alpha+\beta)}{\sin \alpha}
$$

expanding and simplifying we obtain

$$
\left(T_{1}-T_{2}\right)\left(\frac{1}{v_{d}}-\frac{1}{v_{2}}\right)=T_{2}\left(\frac{1}{v_{1}}+\frac{1}{v_{2}}\right),
$$

finally we arrive to

$$
v_{d}=\frac{T_{1}-T_{2}}{T_{1} / v_{2}+T_{2} / v_{1}},
$$

which in the limit case of $v_{1}=v_{2}=v_{w}$ reduces to

$$
v_{d}=v_{w} \frac{T_{1}-T_{2}}{T_{1}+T_{2}} .
$$
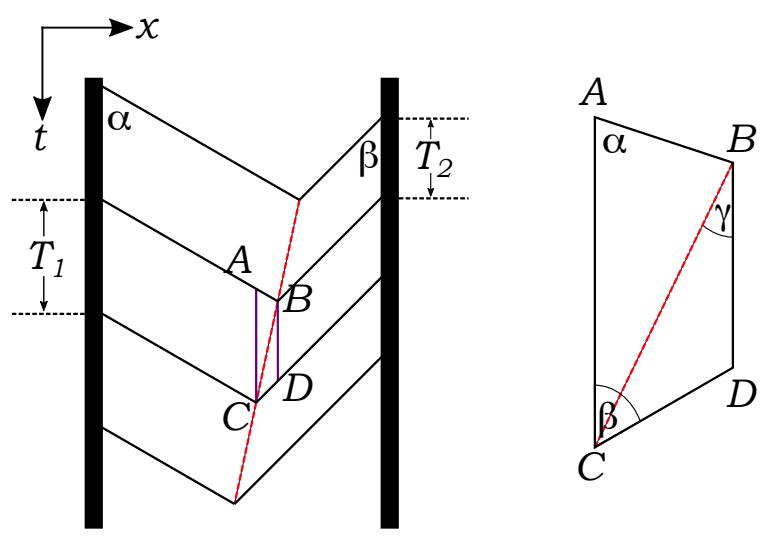

Fig.S 4. Schematic representation in a space-time plot of two waves coming from pillars. Thick lines represent pillars and the red dashed line marks the position of the annihilation point. Waves are emitted from the left pillar with a period $T_{1}$ and with $T_{2}$ from the right pillar. These values correspond to the vertical spacing between waves in this representation.

\section{Numerical simulations with and without caffeine in the presence and absence of pillars}

Simulations in the absence of caffeine with and without the obstacles are shown in Figs. S5 A-D. We also performed numerical simulations with caffeine (larger $K_{m}$ value) but without pillars. As shown in Fig. S6, the number of wave centers is reduced and thereby the streaming domains are larger.

\section{Numerical simulations with higher cell accumulation around the pillars (with caffeine)}

Figure S7 shows the numerical simulations with slightly higher cell density around the obstacles (see also supplemental movie 13).

\section{Numerical simulations with hexagonal and triangular arrangement of pillars (with caffeine)}

We also performed numerical simulations with hexagonal and triangular arrangement of the pillars and obtained triangular and hexagonal arrangements, respectively (see Figs. S8 and S9).

\section{Numerical simulations with $\epsilon$ as the control parameter}

In D. discoideum, activation of ACA is mediated by cAMP receptor, cAR1, that interacts with a G-protein, G2. It is believed that G2 $\beta \gamma$-subunit activates a phospholipid (called inositol kinase) that generates membrane-binding sites for the CRAC, which activates ACA upon recruitment to the plasma membrane (2). It is suggested that in the presence of caffeine the activation of $\mathrm{G} 2$ protein is reduced, thus reducing the number of ACAs in the active form. Thereby, we repeated our numerical simulations with $\epsilon$ (instead of $\left.K_{m}\right)$ as the control parameter. In the MG model, $\epsilon$ controls the ratio of the active to inactive forms of ACA: higher values of $\epsilon$ correspond to lower caffeine concentrations. As shown in Fig. S10, our results are similar to those obtained with $K_{m}$ as the control parameter. 

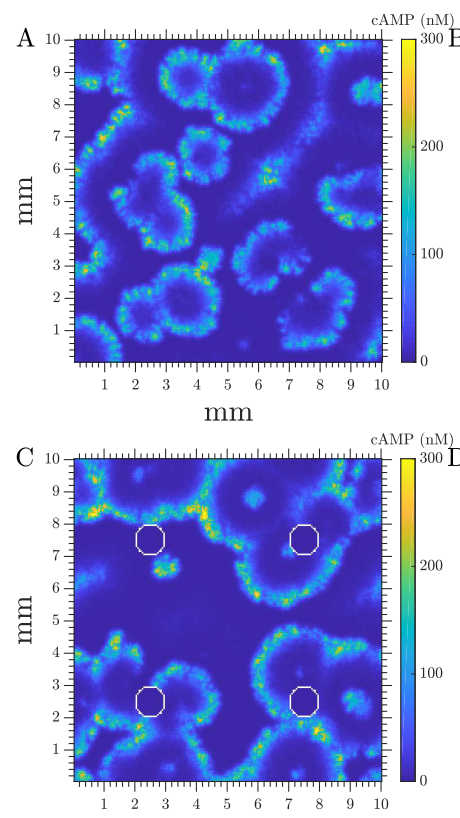

$\mathrm{mm}$

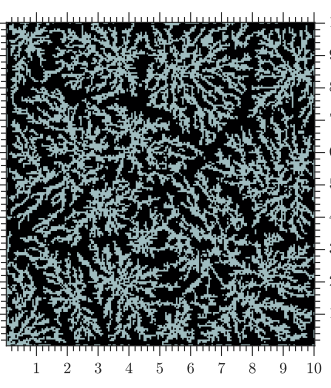

$\mathrm{mm}$

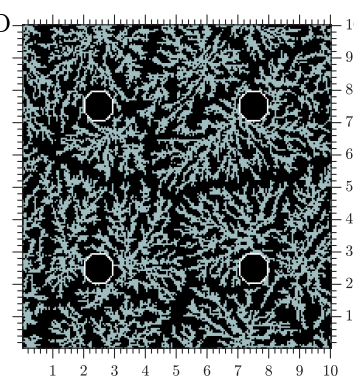

$\mathrm{mm}$

Fig.S 5. Numerical simulations of CAMP waves with and without pillars and no caffeine, $K_{m}=0.40 \mathrm{mM}, k_{e}=5.5 \mathrm{~min}^{-1}, 40 \%$ of cell surface coverage, no flux boundary condition at the pillars boundaries. A) Waves of cAMP at $t=68 \mathrm{~min}$. B) Cell distribution after $t=150 \mathrm{~min}$. Grey squares show grid points containing a cell, black squares show the empty ones. C) Waves of cAMP at $t=49 \mathrm{~min}$. D) Cell distribution after $t=150 \mathrm{~min}$. See also supplementary movies 9 and 10 .
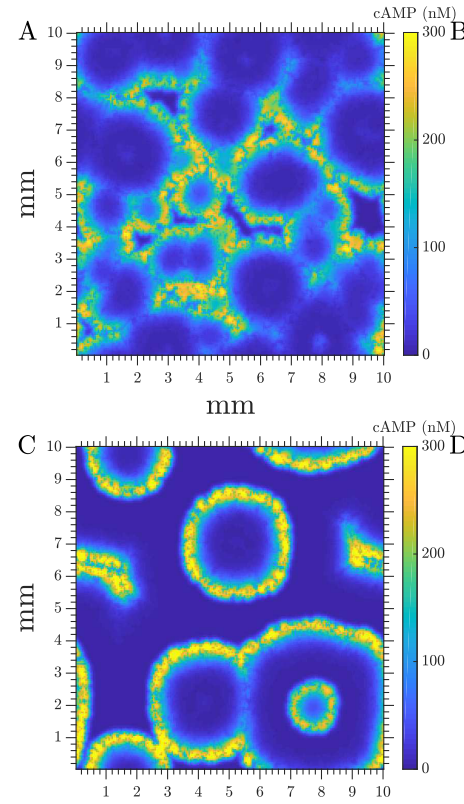

$\mathrm{mm}$

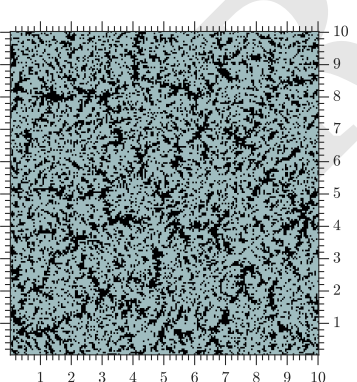

$\mathrm{mm}$

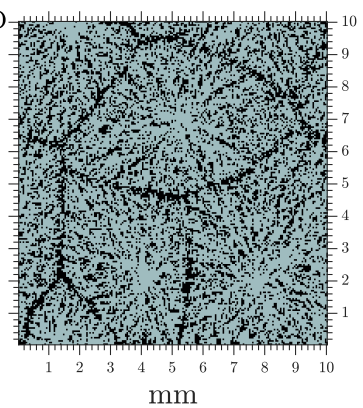

$\mathrm{mm}$

Fig.S 6. Numerical simulations showing the effects in amount of target centers when adding caffeine to $\mathrm{D}$ discoideum. A) cAMP waves without caffeine at $t=68 \mathrm{~min}$ $K_{m}=0.4 \mathrm{mM}$. B) Cell distribution after $t=100 \mathrm{~min}, K_{m}=0.4 \mathrm{mM}$. C) cAMP waves with caffeine at $t=68 \mathrm{~min}, K_{m}=0.48 \mathrm{mM}$. D) Cell distribution after $t=100 \mathrm{~min}, K_{m}=0.48 \mathrm{mM} . k_{e}=6.0 \mathrm{~min}^{-1}, 70 \%$ of cell coverage. (see also movie 12)

$\mathrm{mm}$

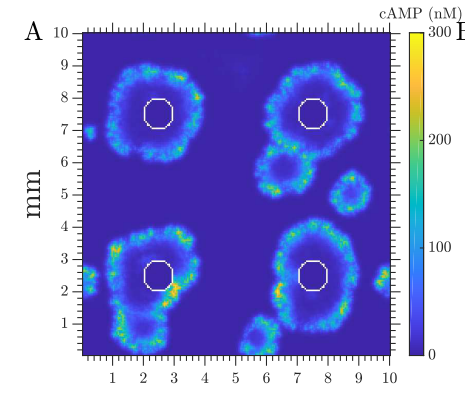

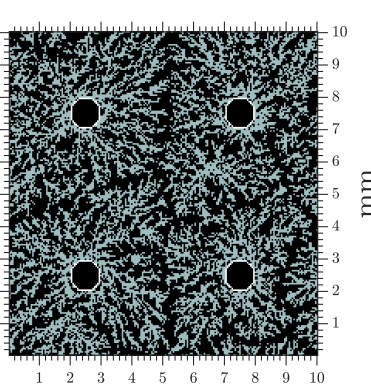

$\mathrm{mm}$
Fig.S 7. Numerical simulation showing the effect of a higher cell accumulation around pillars. $K_{m}=0.48 \mathrm{mM}, k_{e}=5.0 \mathrm{~min}^{-1}, 60 \%$ of cell coverage around the pillar, $40 \%$ in the rest of the system. A) Waves of cAMP at $t=15 \mathrm{~min}$. B) Cell distribution after $t=150 \mathrm{~min}$. Grey squares show grid points containing a cell, black squares show the empty ones (see also movie 13).

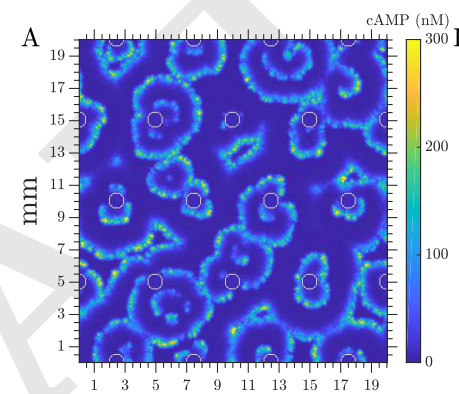

$\mathrm{mm}$

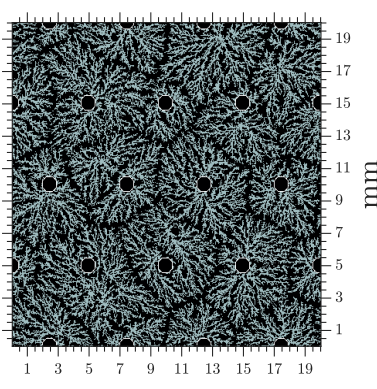

$\mathrm{mm}$
Fig.S 8. Numerical simulations of cAMP waves with a triangular pillar array. Fixed boundary condition $\gamma_{0}=10 \mathrm{nM}, K_{m}=0.508 \mathrm{mM}, k_{e}=5.0 \mathrm{~min}^{-1}, 40 \%$ of cell surface coverage. A) Concentric waves of cAMP coming out of the pillars. B) Cell distribution after $t=120 \mathrm{~min}$, showing Voronoi domains around the pillars. Grey squares show grid points containing a cell, black squares show the empty ones. Pillar boundaries marked in white (see movie 14).

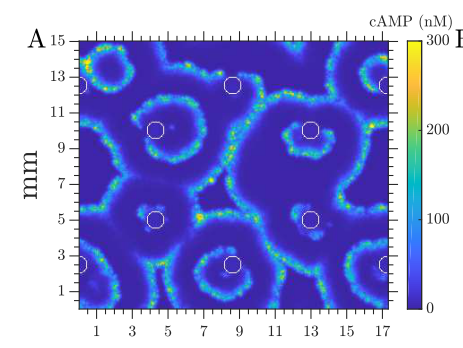

$\mathrm{mm}$

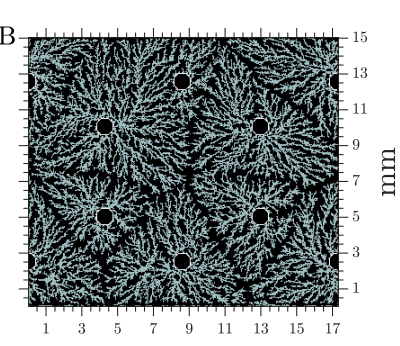

$\mathrm{mm}$
Fig.S 9. Numerical simulations of CAMP waves with a hexagonal pillar array. Fixed boundary condition $\gamma_{0}=10 \mathrm{nM}, K_{m}=0.508 \mathrm{mM}, k_{e}=5.0 \mathrm{~min}^{-1}, 40 \%$ of cell surface coverage. A) Concentric waves of cAMP coming out of the pillars. B) Cell distribution after $t=150 \mathrm{~min}$, showing Voronoi domains around the pillars. Grey squares show grid points containing a cell, black squares show the empty ones. Pillar boundaries marked in white (see movie 15). 

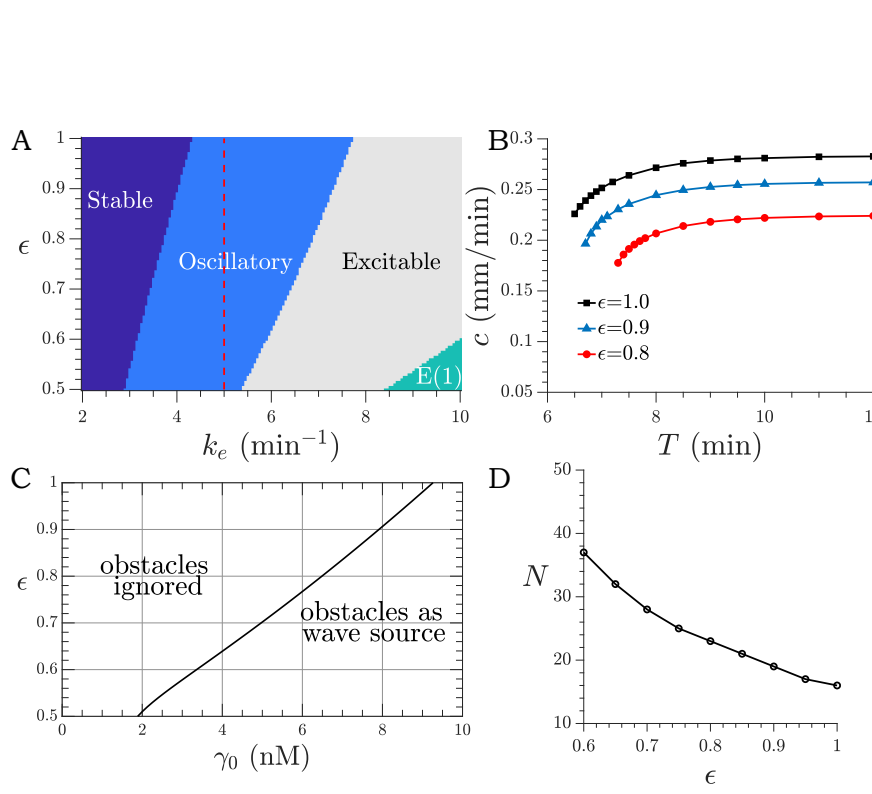

Fig.S 10. A) Phase diagram of the reaction-diffusion model used for simulating the experimental setup. On the area marked as Stable, the system has one solution, which is stable. In the Oscillatory region, the system shows one unstable steady state surrounded by a limit circle. In the Excitable regime, the system has 3 steady states, two unstable ones and a stable one, which is excitable. In the regime marked as $E(1)$ the system shows one steady state, which is excitable. Stability calculated through linear analysis, and excitability through no-space simulations. Red dashed line shows the path that $\epsilon$ is changed in parts $\mathrm{B}, \mathrm{C}$, and $\mathrm{D}$ at fixed value of $k_{e}=$ $5.0 \mathrm{~min}^{-1}$. Note that higher $\epsilon$ values correspond to lower caffeine concentration. B) Dispersion relations of the supported wave trains for $40 \%$ surface coverage, where $T$ is wave period and $c$ wave velocity. Waves with periods below those shown, do not get relayed by the system. C) Effect of a boundary condition $\gamma_{0}$ : for smaller values of $\epsilon$ the minimum amount of $\gamma_{0}$ required to produce a target center decreases. D) Minimum number $N$ of consecutive cells (in 1-D) needed to produce a cluster with self-sustained oscillations.
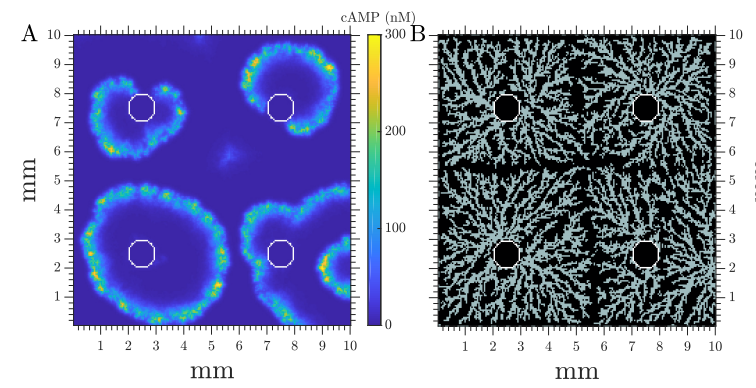

Fig.S 11. Numerical simulations of cAMP waves with four pillars as obstacles with fixed boundary condition $\gamma_{0}=8 \mathrm{nM}, \epsilon=0.78$ (lower $\epsilon$ value to include the effect of caffeine), $k_{e}=5.0 \mathrm{~min}^{-1}$, and $40 \%$ of cell surface coverage. A) Concentric waves of cAMP coming out of the pillars at $t=32 \mathrm{~min}$. B) Cell distribution after $t=150$ min, showing Voronoi domains around the pillars. Grey squares show grid points containing a cell, black squares show the empty ones (see movie 16).
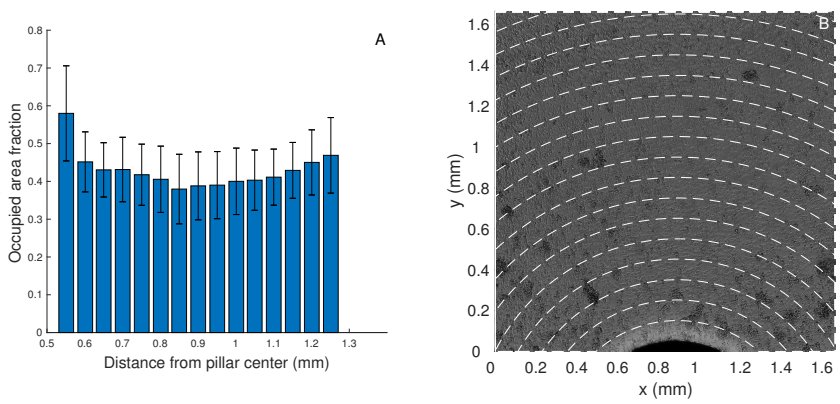

Fig.S 12. Systematic measurements of density around the pillars of diameter $1 \mathrm{~mm}$ show no significant cell accumulation in the vicinity of the obstacles. The distance is measured from center of the pillar and standard deviations are calculated from the measurements for $\sim 11$ pillars. Note that the area around each pillar is divided to circular bands and the occupied area fraction is measured for each band.

\section{Period measurements: Experiments and Simulations}

We measured the wave periods in our simulations as a function of $K_{m}$. Higher $K_{m}$ values correspond to higher caffeine concentrations. Consistent with experiments, we observed an increase in the wave period at higher caffeine concentrations (see Fig. S13 A). We also repeated the measurements for various $\epsilon$ values. Note that higher $\epsilon$ correspond to lower caffeine concentration (see Fig. S13 B).

Figure S14 shows experimental measurements of the wave periods as time evolves for two cases: i) without caffeine and ii) in the presence of $2 \mathrm{mM}$ caffeine.
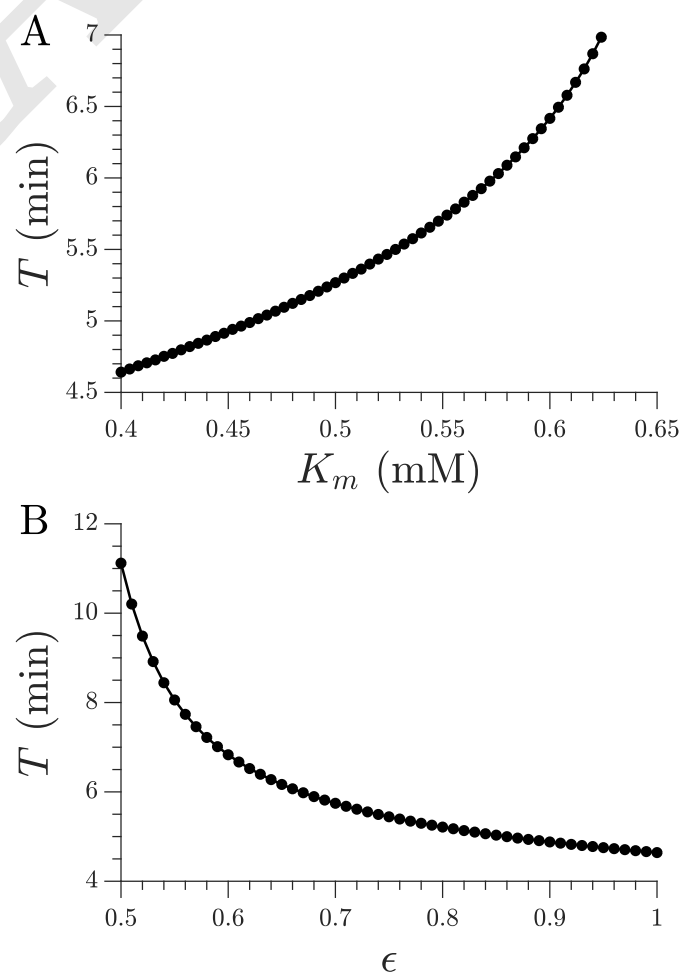

Fig.S 13. Period measurements in the simulations: A) The wave period increases at higher $K_{m}$ values (higher caffeine concentrations). B) The wave period decreases at higher $\epsilon$ values (lower caffeine concentrations). 


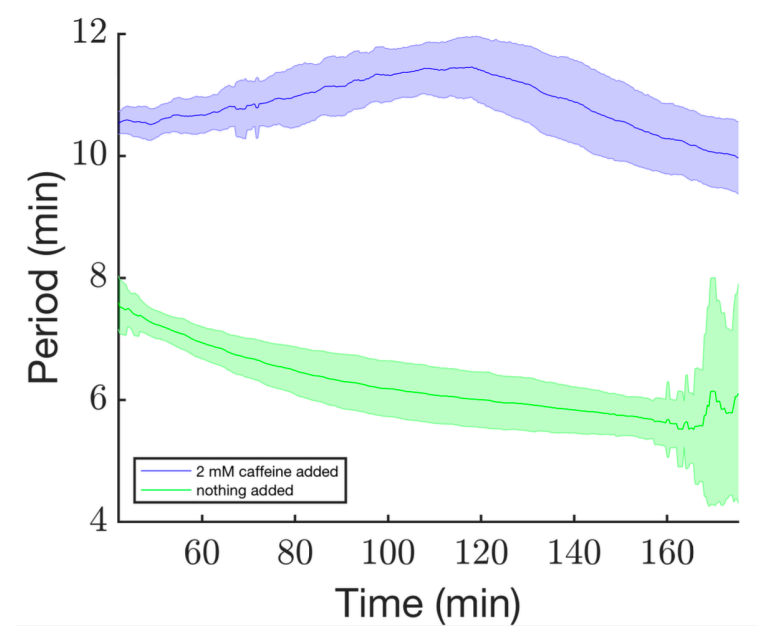

Fig.S 14. Period of cAMP waves as time evolves without and with $2 \mathrm{mM}$ caffeine. For each experiment, 35 measurements are included.
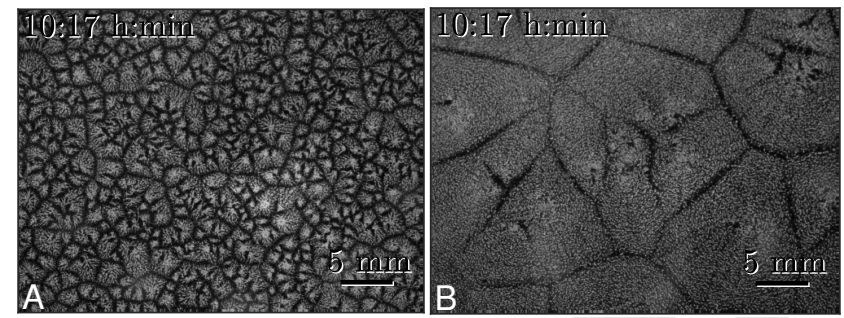

Fig.S 15. Comparison of aggregation territories in two experiments: A) without caffeine and B) with $2 \mathrm{mM}$ caffeine. Consistent with literature (3) and our numerical simulations, in the presence of caffeine, the number of firing centers is reduced. Note that the cells are not initially starved for these two experiments and the substrate is PDMS (see movie 23).

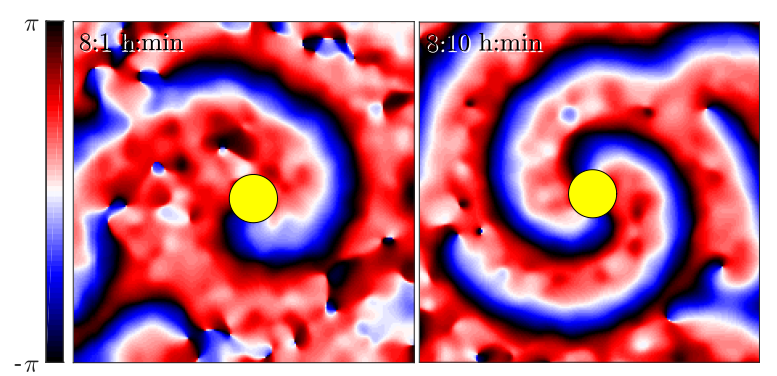

Fig.S 16. Snapshots of pinned single and double arm spirals in two different experiments in the presence of $2 \mathrm{mM}$ and $0.9 \mathrm{mM}$ caffeine, respectively. Double arm spirals are rarely observed and are not stable without an anchoring pillar in our experiments (see also movie 24).

\section{Supplemental Movies}

Movie1 An experiment in the presence of pillars without caffeine. The left panel shows the original dark field movie and the right panel shows the processed movie (subtracted and band-passed filtered) to have a better visualization of the waves.

Movie2 An experiment in the absence of pillars with $2 \mathrm{mM}$ caffeine. Please note that the substrate is PDMS. The left panel shows the original dark field video and the right panel shows the processed movie.

Movie3 Wave propagation, streaming and formation of the Voronoi domains in an array with quadratic arrangement. Caffeine concentration is $2 \mathrm{mM}$.

Movie4 Images in movie 3 are subtracted every 1 min and band-passed filtered to reduce the spatial noise.

Movie5 Phase map calculated using Hilbert transform of filtered images of movie 3 .

Movie6 Concentric waves and regular streaming domains in an experiment with 50 micron high pillars. The other parameters of the array are not changed. The right panel is again processed movie.

Movie7 Experiment with triangular arrangement of the pillars.

Movie8 Experiment with hexagonal arrangement of the pillars.

Movie9 Numerical simulation showing the appearance of target centers and spirals without the addition of caffeine. Spirals marked with a white circle. $k_{e}=5.5 \mathrm{~min}^{-1}, K_{m}=0.4$ $\mathrm{mM}, 40 \%$ of cell coverage.

Movie10 Numerical simulation showing pillars being ignored without the addition of caffeine. $K_{m}=0.40 \mathrm{mM}$ $k_{e}=5.5 \mathrm{~min}^{-1}, 40 \%$ of cell surface coverage, no flux boundary condition at the pillars boundaries.

Movie11 Numerical simulation showing the effects of adding caffeine to $D . d .$. In the presence of caffeine, the pillars act as centers even for a small accumulation of cAMP. Up: Simulation without caffeine, $K_{m}=0.4 \mathrm{mM}$. Down: Simulation with caffeine $K_{m}=0.48 \mathrm{mM} . k_{e}=6.0 \mathrm{~min}^{-1}, 70 \%$ of cell coverage, fixed boundary condition at $\gamma_{0}=6 \mathrm{nM}$.

Movie12 Numerical simulations showing the effects in amount of target centers when adding caffeine to $D . d$. Up: Simulation without caffeine, $K_{m}=0.4 \mathrm{mM}$. Down Simulation with caffeine $K_{m}=0.48 \mathrm{mM}$. $k_{e}=6.0 \mathrm{~min}^{-1}, 70 \%$ of cell coverage.

Movie13 Numerical simulations showing the effect of a higher cell accumulation around pillars. $K_{m}=0.48 \mathrm{mM}$. $k_{e}=5.0 \mathrm{~min}^{-1}, 60 \%$ of cell coverage around the pillar, $40 \%$ in the rest of the system.

Movie14 Numerical simulation showing a triangular array of pillars, producing hexagonal patterns. $k_{e}=5.0$ $\mathrm{min}^{-1}, K_{m}=0.508 \mathrm{mM}, 40 \%$ of cell coverage, fixed boundary condition at $\gamma_{0}=10 \mathrm{nM}$.

Movie15 Numerical simulation showing a hexagonal array of pillars, producing triangular patterns. $k_{e}=5.0$ $\min ^{-1}, K_{m}=0.508 \mathrm{mM}, 40 \%$ of cell coverage, fixed boundary condition at $\gamma_{0}=10 \mathrm{nM}$

Movie16 Numerical simulations of cAMP waves with four pillars as obstacles with fixed boundary condition $\gamma_{0}=8$ $\mathrm{nM}, \epsilon=0.78$ (to include the effect of caffeine), $k_{e}=5.0 \mathrm{~min}^{-1}$, and $40 \%$ of cell surface coverage.

Movie17 Bright field movie of a single post in a quadratic array of the pillars.

Movie18 Experiment with pillars of $1.5 \mathrm{~mm}$ in diame- 
ter. Center-to-center distance is not changed $(5 \mathrm{~mm})$.

Movie19 Experiment with shallow holes (100 $\mu \mathrm{m}$ deep) holes in PDMS.

Movie20 Experiment with a flat wall as obstacle. The wall thickness is $1 \mathrm{~mm}$.

Movie21 Numerical simulation showing the effect of lower degradation around pillars. $k_{e}=4.0 \mathrm{~min}^{-1}$ around pillars, $k_{e}=5.0 \mathrm{~min}^{-1}$ on the rest of the system. $K_{m}=0.48$ mM. $40 \%$ of cell coverage.

Movie22 Numerical simulation showing how pillars are ignored when there is a higher degradation rate around the pillars. $k_{e}=6.0 \mathrm{~min}^{-1}$ around pillars, $k_{e}=5.0 \mathrm{~min}^{-1}$ on the rest of the system. $K_{m}=0.48 \mathrm{mM}$. $40 \%$ of cell coverage.
Movie23 Comparison of two experiments without (left) and with $2 \mathrm{mM}$ caffeine (right). Note that for both experiments, the cells are not initially starved in a shaking suspension and the substrate is PDMS.

Movie24 Two different experiments in the presence of $2 \mathrm{mM}$ and $0.9 \mathrm{mM}$ caffeine showing single and double arm spirals pinned to the pillars.

1. Prabhakara KH, Gholami A, Zykov VS, Bodenschatz E (2017) Effects of developmental variability on the dynamics and self-organization of cell populations. New Journal of Physics 19(11):113024.

2. Alvarez-Curto $E$, Weening KE, Schaap $P$ (2007) Pharmacological profiling of the dictyostelium adenylate cyclases aca, acb and acg. Biochemical Journal 401(1):309-316.

3. Siegert F, Weijer C (1989) Digital image processing of optical density wave propagation in dictyostelium discoideum and analysis of the effects of caffeine and ammonia. Journal of Cell Science 93(2):325-335. 


\section{$5 \quad$ Spontaneous Center Formation in Dictyostelium discoideum}

The following paper originally appeared as Vidal-Henriquez, E. \& Gholami, A. (2019). Spontaneous center formation in Dictyostelium discoideum. Scientific Reports 9(1), 3935. doi: 10.1038/s41598-019-40373-4. It is licensed under CC BY 4.0.

EVH designed and performed the numerical simulations. EVH conducted the theoretical calculations and analysis. EVH and AG designed and planned research. EVH and AG wrote, edited, and reviewed the manuscript. All figures and electronically available movies by EVH. 


\title{
SCIENTIFIC REP
}

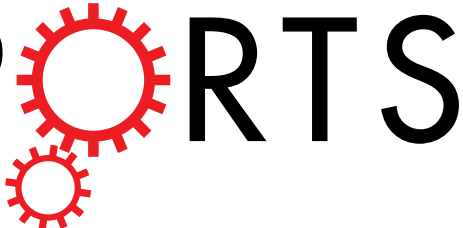

\section{OPEN Spontaneous center formation in Dictyostelium discoideum}

\author{
Estefania Vidal-Henriquez \& Azam Gholami
}

Received: 27 September 2018 Accepted: 8 February 2019 Published online: 08 March 2019
Dictyostelium discoideum (D.d.) is a widely studied amoeba due to its capabilities of development, survival, and self-organization. During aggregation it produces and relays a chemical signal (cAMP) which shows spirals and target centers. Nevertheless, the natural emergence of these structures is still not well understood. We present a mechanism for creation of centers and target waves of CAMP in D.d. by adding cell inhomogeneity to a well known reaction-diffusion model of cAMP waves and we characterize its properties. We show how stable activity centers appear spontaneously in areas of higher cell density with the oscillation frequency of these centers depending on their density. The CAMP waves have the characteristic dispersion relation of trigger waves and a velocity which increases with cell density. Chemotactically competent cells react to these waves and create aggregation streams even with very simple movement rules. Finally we argue in favor of the existence of bounded phosphodiesterase to maintain the wave properties once small cell clusters appear.

Dictyostelium discoideum (D.d.) is a social amoeba that under adverse environmental conditions begins a developmental program in order to survive ${ }^{1}$. After 4-5 hours into this program, the cells start to produce the signaling chemical cAMP (cyclic adenosine monophosphate), which travels the system as a wave relayed by the cells. The cells are chemotactically competent and move against the traveling wave of cAMP towards the aggregating centers. In the centers the cells form a mound, where they further develop and differentiate into a slug and eventually into a fruiting body who releases spores which can become myxamoebas; thus completing the life cycle of the amoeba.

The chemical signaling part of the process presents spirals and target centers, these structures are characteristic of both oscillatory and excitable systems, and are present in many systems in nature such as the cardiac muscle ${ }^{2}$, calcium waves in oocytes ${ }^{3}$, NADH waves in glycolysis ${ }^{4}$, among others. Other important system where such structures are observed is the chemical reaction known as Belousov-Zhabotinsky ${ }^{5,6}$, which has become a model experimental system to study spirals and target waves.

A distributed oscillatory system would produce bulk oscillations if it is well stirred, that is, the whole system oscillates synchronously. If it is not stirred and presents some inhomogeneities, such as scratches on the container surface or dust particles, concentric circular waves will appear ${ }^{7,8}$. We refer to these structures as target patterns. Once a wave breaks, it creates a spiral wave, a persistent structure with topological charge ${ }^{9,10}$.

Many models have been proposed to describe the cAMP waves in $D . d .{ }^{11-13}$, from qualitative excitable models to models derived from the chemical reactions of production of cAMP from ATP. Each model has their own particular way of breaking the homogeneity and producing waves. Here we present the properties of the model proposed by Martiel and Goldbeter ${ }^{13,14}$ when cell inhomogeneity is introduced. We show through numerical simulations that cell inhomogeneity produces naturally stable target patterns which are centered in areas of higher cell density and relayed in the areas of lower density. Therefore, the system behaves both as oscillatory and excitable depending on local density. The produced waves are shown to be of the trigger kind, in contrast to phase waves (or pseudo-waves, without chemical transport), through their characteristic dispersion relation ${ }^{15}$. We also show that wave speed is density dependent. It has been shown in other chemotaxis models that this dependence is enough to produce the wave instability responsible for cell streaming once cell movement is added ${ }^{16,17}$. Our model reproduces that characteristic streaming pattern of this biological system. Finally we show that a form of degradation which scales with local cell density is needed to have streaming at higher local densities, this degradation can exist in the form of phosphodiesterase bounded to the cell membrane.

Max Planck Institute for Dynamics and Self-Organization, Am Fassberg 17, D-37077, Göttingen, Germany. Correspondence and requests for materials should be addressed to E.V.-H. (email: estefania.vidal@ds.mpg.de) or A.G. (email: azam.gholami@ds.mpg.de) 


\begin{tabular}{|l|l|l|}
\hline$c=10$ & $h=5$ & $k 1=0.09 \mathrm{~min}^{-1}$ \\
\hline$\kappa=18.5$ & $\sigma=0.55 \mathrm{~min}^{-1}$ & $k i=1.7 \mathrm{~min}^{-1}$ \\
\hline$k_{t}=0.9 \mathrm{~min}^{-1}$ & $\mathcal{L}_{1}=10$ & $\mathcal{L}_{2}=0.005$ \\
\hline$q=4000$ & $\lambda_{1}=10^{-4}$ & $\lambda_{2}=0.2575$ \\
\hline$\theta=0.01$ & $D=0.024 \mathrm{~mm}^{2} / \mathrm{min}$ & $r=10 \mu \mathrm{m}$ \\
\hline$a_{0}=0.04423$ & $a_{1}=0.1526$ & $a_{2}=328.2$ \\
\hline$v_{c}=0.02 \mathrm{~mm} / \mathrm{min}$ & $\rho_{c}=0.6$ & $\Delta \gamma_{c}=25.98 \mathrm{nM} / \mathrm{mm}$ \\
\hline
\end{tabular}

Table 1. Parameters used for simulations of equation (1) in the first row. Second row: Parameters used to approximate the production function in equation (2). Third row: Parameters used when cell movement was included.

\section{Results}

Our work combines a cellular automata scheme with the model proposed by Martiel and Goldbeter ${ }^{13}$ and extended by Tyson ${ }^{14}$ where three fields are described. The extracellular concentration of cAMP is represented as $\gamma$, the intracellular concentration as $\beta$, and $\rho$ represents the percentage of active receptors on the cell membrane. The receptors' state changes between an active and an inactive state depending on the cAMP concentration at which they are exposed through the functions $f_{1}$ and $f_{2}$. The intracellular concentration of cAMP $\beta$ increases through production $\Phi(\rho, \gamma)$ and decreases through internal degradation $k_{i}$ and transport towards the extracellular media $k_{t}$. Finally the external concentration of cAMP can diffuse through the system, gets degraded by phosphodiesterase $k_{e}$ and increases due to transport from the internal media $k_{t}$.

We created a grid in our 1-D or 2-D system, dividing it in segments of size $r=10 \mu \mathrm{m}$ (or respectively squares of area $r^{2}$ ) and assigned random locations for the cells, each cell had their own value for the intracellular and membrane bound variables $\rho_{i}$ and $\beta_{i}$, while $\gamma(x, y)$ was assigned to a square in space. The used equations look as follows

$$
\begin{gathered}
k_{1}^{-1} \partial_{t} \rho_{i}=-f_{1}\left(\gamma\left(x_{i}, y_{i}\right)\right) \rho_{i}+f_{2}\left(\gamma\left(x_{i}, y_{i}\right)\right)\left(1-\rho_{i}\right), \\
\partial_{t} \beta_{i}=s \Phi\left(\rho_{i}, \gamma\left(x_{i}, y_{i}\right)\right)-\left(k_{i}+k_{t}\right) \beta_{i}, \\
\partial_{t} \gamma(x, y)=D \nabla^{2} \gamma(x, y)-k_{e} \gamma(x, y)+\sum_{i}^{N} H(i, x, y) k_{t} \beta_{i} / h,
\end{gathered}
$$

$$
\text { with } f_{1}(\gamma)=\frac{1+\kappa \gamma}{1+\gamma}, \quad f_{2}(\gamma)=\frac{\mathcal{L}_{1}+\kappa \mathcal{L}_{2} c \gamma}{1+c \gamma}, \quad \Phi(\rho, \gamma)=\frac{\lambda_{1}+Y^{2}}{\lambda_{2}+Y^{2}}, \quad \text { and } Y(\gamma, \rho)=\frac{\rho \gamma}{1+\gamma},
$$

where $s=q \sigma \alpha /(1+\alpha)$ measures the production intensity, $\left(x_{i}, y_{i}\right)$ correspond to the Cartesian coordinates of the $i$-th cell, and $H$ is an indexing function such that $H(i, x, y)=1$ if $x_{i} \in(x-r / 2, x+r / 2)$ and $y_{i} \in(y-r / 2, y+r / 2)$ and 0 otherwise. In this way $\gamma$ will increase on a grid space if there is a cell producing cAMP in that location and only diffuse and be degraded if there are no cells in that space. All used parameter are selected following Lauzeral et al. ${ }^{18}$ for their good agreement with experimental measures and are indicated in Table 1 . We keep $k_{e}$ the external media degradation rate as our control parameter.

The original homogeneous system studied by Tyson $e t$ al. ${ }^{14}$ presented different regimes as $k_{e}$ was increased. In our set of parameters for low $k_{e}$ the system has one steady state which is stable. At $k_{e}^{*} \approx 4.3 \mathrm{~min}^{-1}$ the system undergoes a Hopf bifurcation and a stable limit cycle appears. This is the oscillatory regime of the system. Upon further increasing $k_{e}$ two new steady states appear through a saddle-node bifurcation at $k_{e}^{\dagger} \approx 7.74 \mathrm{~min}^{-1}$, an unstable one and a stable but excitable one. At approximate the same time the limit cycle destabilizes, resulting in the excitable regime of the system. We set our parameter $k_{e}$ so that the system is in the oscillatory regime. For a detailed description of the different regimes present in this system refer to our previous work ${ }^{19}$.

Oscillatory clusters. Numerical simulations of a small cluster of very closely located cells producing cAMP surrounded by buffer media without cells, reach a stable steady (non oscillatory) state due to cAMP diffusion to its surroundings. This state is shown in Fig. 1a. To approximate this solution we calculate separately the area with cells and the area without them. In the area without cells the system reduces to

$$
\partial_{t} \gamma=D \partial_{x x} \gamma-k_{e} \gamma
$$

which in 1-D has a time independent decaying tail as solution. If the cluster size is $2 L$, with the cells located in $x \in(-L, L)$ the decaying tail takes the form

$$
\gamma=A e^{-\sqrt{k_{e} / D}|x|} \quad \text { if }|x|>L
$$

where $A$ is chosen to fulfill the boundary condition at $x= \pm L$, which are continuity, $\gamma\left( \pm L^{-}\right)=\gamma\left( \pm L^{+}\right)$, and continuity of the derivative, $\partial_{x} \gamma\left( \pm L^{-}\right)=\partial_{x} \gamma\left( \pm L^{+}\right)$. 

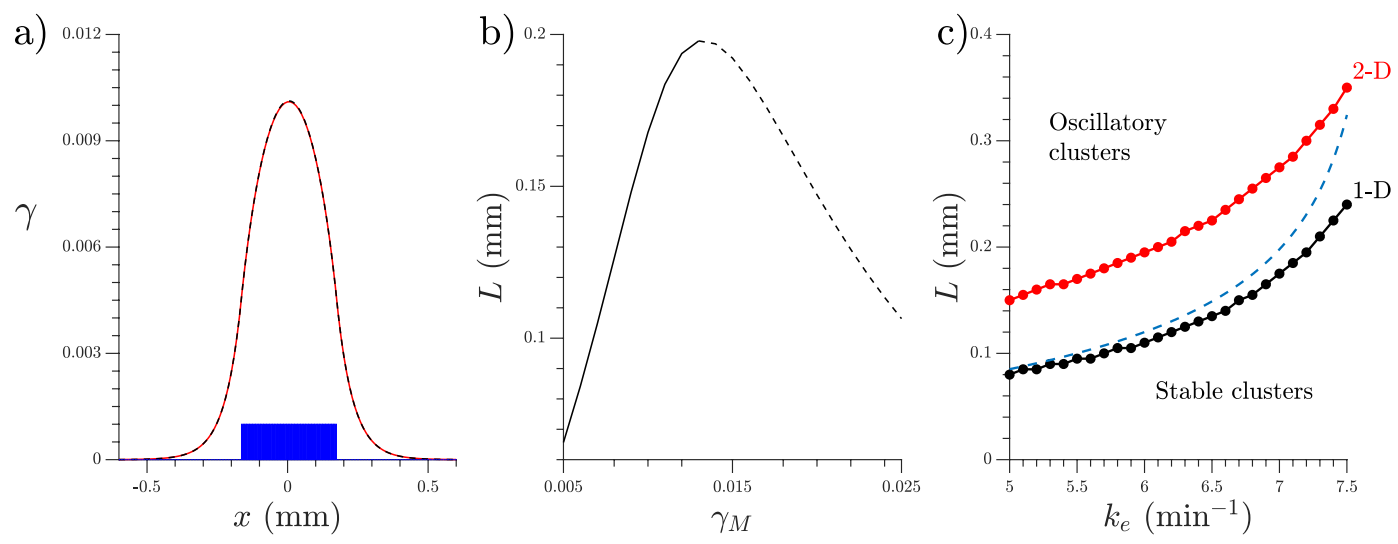

Figure 1. (a) Time independent cluster size solution. Spaces occupied by cells in blue, result of numerical simulations in red, and theoretical prediction in black, dashed line. $k_{e}=7.0 \mathrm{~min}^{-1}$ and $L=0.17 \mathrm{~mm}$, equivalent to 34 consecutive cells. (b) Distance $L$ from the center at which the system matches the decaying tail boundary condition, depending on the maximum concentration $\gamma_{M}$ reached at $x=0$. Stable solution in continuous line and unstable in dashed line. $k_{e}=7.0 \mathrm{~min}^{-1}$. (c) Maximum size at which cluster still remain non-oscillatory. 1-D simulations in black, 2-D in red, theoretical 1-D prediction in dashed line.

For the cAMP concentration inside the cell cluster we used the fact that simulation results showed that the $\gamma$ values inside the cluster are small compared to the values of the cAMP waves, which justifies making an approximation of the production function $\Phi$ for small values of $\gamma$, we therefore take the polynomial approximation $k_{t} \beta / h \approx a_{0}+a_{1} \gamma+a_{2} \gamma^{2}$ and the system reduces inside the cluster to

$$
0=a_{0}+\left(a_{1}-k_{e}\right) \gamma+a_{2} \gamma^{2}+D \partial_{x x} \gamma
$$

It is worth mentioning that the polynomial approximation values were not taken from the Taylor expansion of the production function for $\gamma \ll 1$, but rather from a least square fitting of the production function. The fitted values for our set of parameters (independent of $k_{e}$ ) are listed in Table 1. Equation (2) just by itself is invariant to space translation, even though this is not true for the entire system, this invariance produces a conserved quantity, which we will call energy and can be used to calculate the shape of the cAMP accumulated around the cluster. We can rearrange the equation to show its energy conservation more clearly

$$
\partial_{x x} \gamma=-\frac{\partial V}{\partial \gamma} \quad \text { with } \quad V=\frac{a_{0}}{D} \gamma+\frac{\left(a_{1}-k_{e}\right)}{2 D} \gamma^{2}+\frac{a_{2}}{3 D} \gamma^{3}
$$

and the total energy $E$ can be found by integrating both sides by $\gamma$. Therefore $E=V+\left(\partial_{x} \gamma\right)^{2} / 2$ is conserved and fixed by the boundary condition. To calculate the shape of the solution we need the energy value, but since the decaying tail has a free parameter we need to introduce a second free parameter and then match the two solutions. Therefore, we leave the maximum value of $\gamma, \gamma_{M}$ as a free parameter and we numerically calculated the solution using

$$
\int_{\gamma}^{\gamma_{M}} \frac{d \gamma}{\sqrt{2\left[E\left(\gamma_{M}\right)-V(\gamma)\right]}}=x
$$

For every $\gamma_{M}$ there is a size $L$ such that it fits the boundary condition to match the decaying tail

$$
\sqrt{\frac{k_{e}}{D}} \gamma(x=L)=\left.\partial_{x} \gamma\right|_{x=L}=\sqrt{2(E-V(x=L))},
$$

an example of the relation $\gamma_{M}$ vs $L$ is shown in Fig. 1b. From there, it can be seen that it exists a maximum length for which the static solution exists. For those values of $L$ where two possible values of $\gamma_{M}$ exist, the system chooses the one with smaller $\gamma_{M}$. Indeed linear stability analysis showed that the smaller solution is stable, while the bigger one is unstable. Following this procedure we arrive at two results. First the cluster size values at which a stable, time independent solution exists, and second an approximation of this solution when it exists, see for comparison Fig. 1a where both the numerical solution and theoretical prediction are plotted with excellent agreement.

The predicted maximum cluster size for different values is compared to the biggest clusters found through numerical simulations in one and two dimensions in Fig. 1c with good agreement for small $k_{e}$. We believe the difference in agreement for large $k_{e}$ comes from the potential $V$ approximation, which is valid only for low values of $\gamma$, since for larger $k_{e}$ the values of $\gamma$ are higher, the approximation is not so good, but still manages to catch the general behavior of the system. For 2-D simulations, cells where located in $r<L$ with $r^{2}=x^{2}+y^{2}$. We see that bigger clusters can be maintained in a low steady state when the degradation is increased, this is expected since for fixed $L, \gamma_{M}$ diminishes with increasing $k_{e}$. 

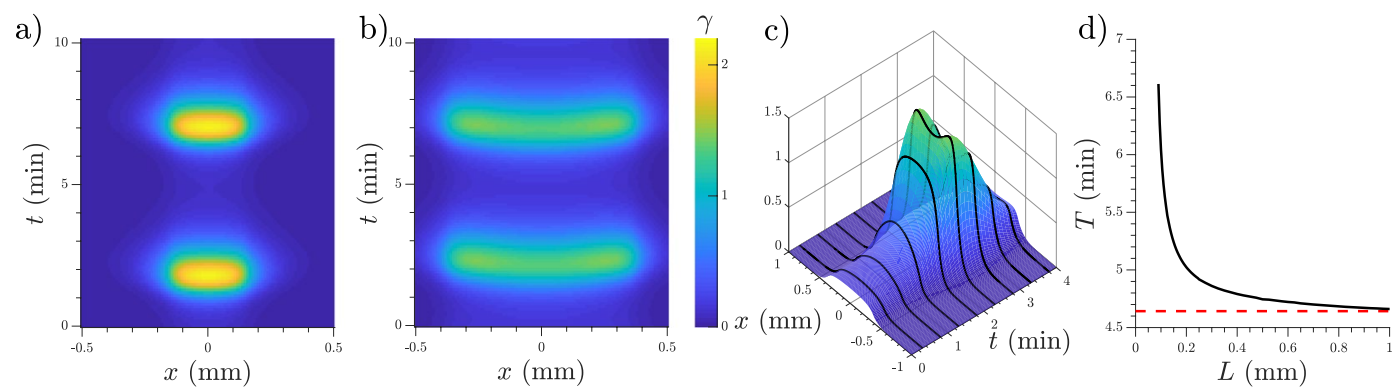

Figure 2. (a) Space-time visualization of a self-oscillating cluster, with cells in $|x|<0.15 \mathrm{~mm} . k_{e}=5.0 \mathrm{~min}^{-1}$. (b) A larger cluster of cells with $L=0.375 \mathrm{~mm}$ and $k_{e}=5.0 \mathrm{~min}^{-1}$. (c) Different representation of the simulation in (b), concentration of cAMP is shown in the $z$ axis with equitemporal lines to guide the view. The increase in amplitude towards the cluster edges can be observed (see also Supplementary Video S2). (d) Oscillation period of isolated clusters depending on their size in black line. Cells located in $|x|<L$ and rest of the system empty space, $k_{e}=5.0 \mathrm{~min}^{-1}$. Red dashed line marks the period of the limit cycle in spaceless $(0-\mathrm{D})$ simulations.
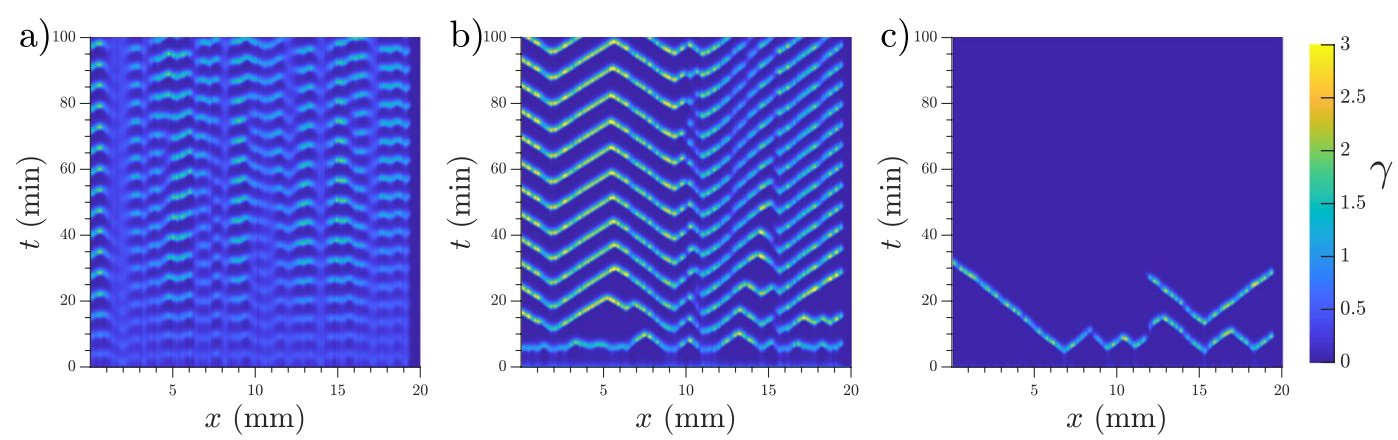

Figure 3. Patterns at different degradation rates. Patterns showed by the system with a random cell distribution at a density of $5 \cdot 10^{5}$ cells $/ \mathrm{cm}^{2}\left(0.5\right.$ mono-layer) and different degradation rates. (a) $k_{e}=4.0 \mathrm{~min}^{-1}$, (b) $k_{e}=5.5 \mathrm{~min}^{-1}$, and $(\mathbf{c}) k_{e}=7.0 \mathrm{~min}^{-1}$.

If the cell group is bigger than its critical size it oscillates at a frequency that is size dependent, see for example Fig. 2a, also depicted in Supplementary Video S1, where one oscillating cluster is shown. As the cell group becomes larger, the frequency increases converging towards the limit cycle frequency. We measured this value for different cluster sizes once the cluster frequency converged to measuring precision. This relation is shown in Fig. 2d.

At small sizes the cluster oscillation is synchronized (bulk), but as it gets bigger $(L \gtrsim 0.19 \mathrm{~mm})$ a wave develops. This wave starts at the center of the cluster and moves towards the sides increasing its amplitude as it travels, as can be seen in Fig. 2b,c. Compare to Fig. 2a which shows a smaller cluster which oscillates synchronously. This effect can also be seen in Supplementary Video S2 which in turn can be compared to Supplementary Video S1.

Target patterns in random distributions of cells. When we assigned random locations to the cells, the system showed different regimes as we increased $k_{e}$. For low values of $k_{e}$ the system oscillated mostly synchronously, see Supplementary Video S3 and Fig. 3a) for a space-time plot. For high values of $k_{e}$ the system was incapable of oscillating by itself and reached a steady state of low cAMP (see Fig. 3c).

A more interesting behaviour was observed for intermediate values of $k_{e}$. There, the areas of higher local density acted as oscillatory centers, while the rest of the system relayed the emitted waves. This means that the lower density areas although not capable of oscillating by themselves are capable of producing enough cAMP to maintain the wave and avoid its complete degradation.

This heterogeneity in the system response, seemingly both oscillatory and excitable, appeared even though the same parameters were used trough the whole system, the heterogeneity being given by the cell distribution.

The appeared target centers have a range of frequencies and are stable. They also interact with each other, thus higher frequency centers dominate over the lower frequency ones. These characteristics match the observation in D.d. patterns ${ }^{20}$. A typical example of our simulation results can be seen in Fig. 3b and in Supplementary Video S4.

The range of degradation values $k_{e}$ in which centers can be observed depends on cell density as is shown in Fig. 4a. Under the shaded area bulk oscillations like in Fig. 3a are observed, and above the shaded area spontaneous center do not appear, like in Fig. 3c. At higher cell densities, higher degradation rates are required to observe spontaneous centers, which is consistent with the idea that phosphodiesterase is produced and released to the external media by the cells, therefore a higher concentration of cells should produce a higher concentration of phosphodiesterase and therefore a faster degradation. Nevertheless, the amount of maximum degradation does not scale linearly with density, but the size of the existence range decreases with higher density. 

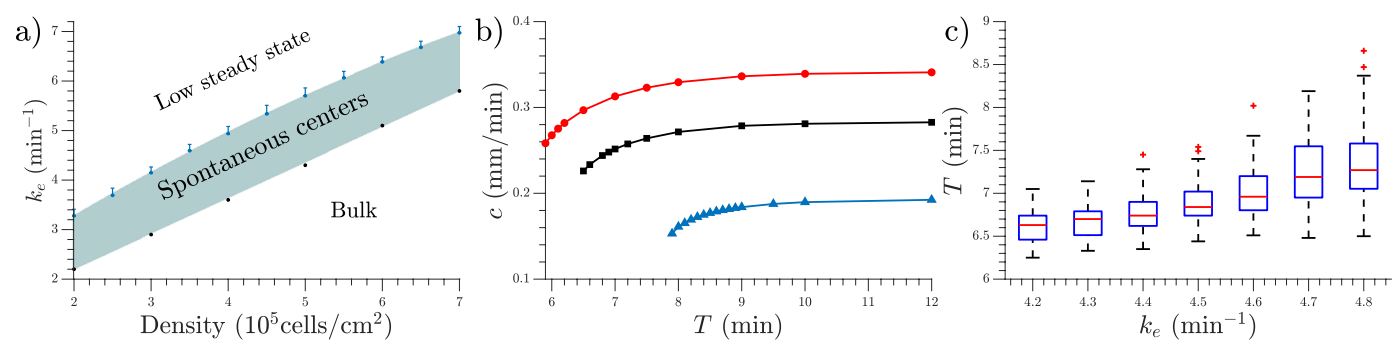

Figure 4. (a) The shaded area indicates where spontaneous centers can be observed, depending on cell density. Blue dots indicate the degradation rate at which $75 \%$ of the simulations presented spontaneous centers. Error bar extends up to $50 \%$. Lower boundary marks the maximum degradation rate at which the homogeneous system presents bulk oscillations. (b) Dispersion relations for different densities. $5 \cdot 10^{5} \mathrm{cells} / \mathrm{cm}^{2}(0.5$ monolayer) in red circles, $4 \cdot 10^{5} \mathrm{cells} / \mathrm{cm}^{2}(0.4$ mono-layer $)$ in black squares, and $3 \cdot 10^{5} \mathrm{cells} / \mathrm{cm}^{2}(0.3$ mono-layer $)$ in blue triangles. $k_{e}=5.0 \mathrm{~min}^{-1}$. (c) Center frequency for different degradation values. 1-D simulations with density $=4 \cdot 10^{5}$ cells $/ \mathrm{cm}^{2}, 100$ simulations used for each $k_{e}$ value.

The transitions between synchronized oscillations, center creation, and low steady state are not bifurcations but rather smooth and of a stochastic nature. That means, for example, that for high values of $k_{e}$ a small number of centers may still appear in some simulations if by chance big clusters of cells appear together. The shaded area of Fig. 4a indicates where centers spontaneously appear in more than $75 \%$ of the cases. See Methods for a description of the boundary calculations for Fig. 4a.

To understand the nature of the traveling waves we calculated their dispersion relation, i.e. the shape of the velocity-period curve. We used the homogeneous distribution simulations mentioned in Methods to study more controlled waves. The waves are generated perturbing the system with a period $T$ and the velocity is calculated following each individual peak once they passed the initial transient. These dispersion relations are presented in Fig. $4 \mathrm{~b}$. It can be seen that they present the characteristic shape of trigger waves ${ }^{15}$, therefore they are actual waves that produce transport of chemicals in contrast to pseudo- or phase waves which do not involve chemical transport and have a smaller dependence on diffusion. Here, diffusion is necessary to activate the next cell and with that relay the wave, another characteristic of trigger wave behaviour.

If the homogeneous system is perturbed with higher frequencies than those depicted in Fig. $4 \mathrm{~b}$ it will not react with a 1:1 response, but instead it will propagate the waves with a lower frequency, usually located in the elbow (strong curvature) area of the curve.

Furthermore, we measured the frequency of the signaling centers in 1-D simulations for a fixed cell density of $4 \cdot 10^{5}$ cells $/ \mathrm{cm}^{2}$ (0.4 mono-layer) as a function of degradation rate. We measured lower center frequencies at higher degradation rates, as shown in Fig. 4c.

Streaming. One common test for the waves generated in a D.d. model is if the system is capable of showing streaming once cell movement is added. Streaming is a characteristic feature of the intermediate state of D.d. on their way towards the mounds. It consist on the alignment of the cells in a head to tail manner, thus displaying long ramified lines of higher density that spread radially from the aggregating center.

To test this feature, we added cell movement in a simple toy-like manner. The cells would move at a constant speed $v_{c}$ if they sense a cAMP gradient bigger than a minimum $\partial_{x} \gamma>\Delta \gamma_{c}$ and if their percentage of active receptors is bigger than a cutoff $\rho>\rho_{c}$. The first rule avoids movement due to random noise and the second avoids the back-of-the-wave problem ${ }^{21,22}$. The back-of-the-wave paradox consists in taking into account that $D$.d. only moves in the first half of the wave, ignoring the gradient of the decreasing cAMP in the second half which would move the cell in the opposite direction. By adding a minimum $\rho$ to allow the cells to move, they effectively move only in the first part due to the desensitization produced by the passing wave. The cells continue moving as long as these both conditions are met. Initially we did not allowed cell superposition, therefore a cell would only move towards a different space if the arriving location were empty. The updated cell positions were calculated each time step using a forward Euler scheme, after the $\gamma, \beta$, and $\rho$ fields were calculated. All used parameter are listed in Table 1.

We performed numerical simulations in 2-D where we allowed cell movement after a pattern was already established $(t \approx 50 \mathrm{~min})$. Images of a typical simulation are presented in Fig. 5 where the streams can be clearly observed, thus recovering the expected behavior. The full simulation can be seen in Supplementary Video S5. It is also worth mentioning that after cells start to move some wave fronts may break and produce spirals.

Cell superposition and bounded phosphodiesterase. As a way of perfecting our simple toy model for movement we included the possibility of cells superimposing in the same location, with a maximum of 5 cells per grid point. In order for the system to continue presenting centers and continuous cell streaming, additional degradation is needed. That is, with the previously used constant degradation the centers and aggregation streams start to break once cell clumps appear due to movement. See Fig. 6 in comparison to Fig. 5 where target centers and stream lines quickly break apart. A simulation showing this behaviour is presented in Supplementary Video S6.

Many experimental studies point to the existence of phosphodiesterase bounded to the cell membrane ${ }^{23,24}$. Adding this extra bounded phosphodiesterase $k_{e B}$ solves these problems and the system behaves as before, 
a) 10

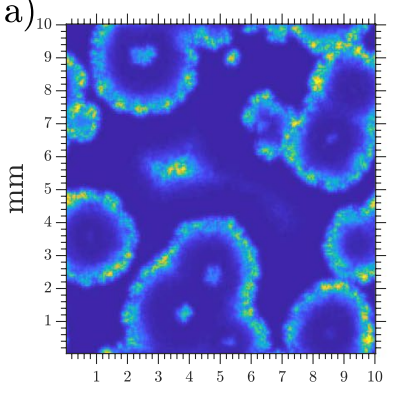

$\mathrm{mm}$

c)

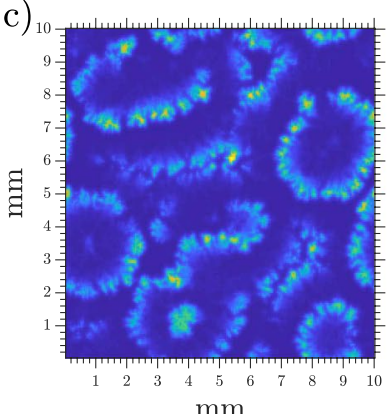

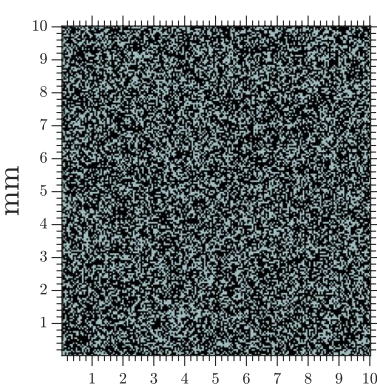

$\mathrm{mm}$

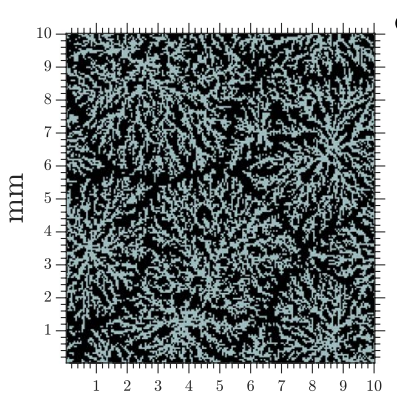

$\mathrm{mm}$

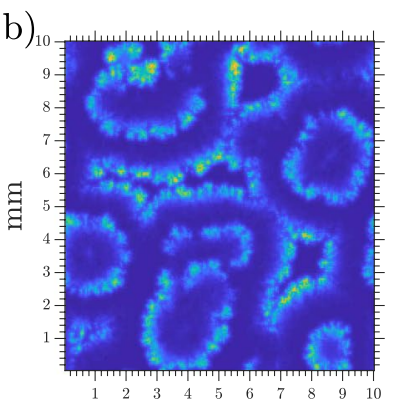

$\mathrm{mm}$

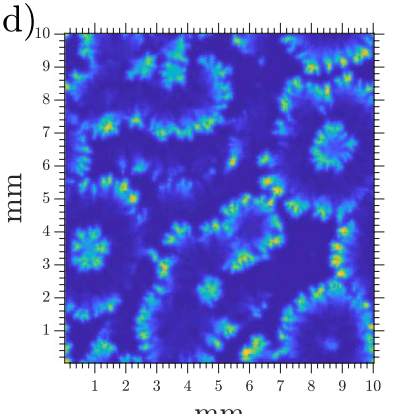

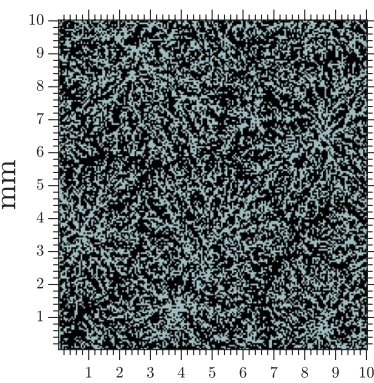

$\mathrm{mm}$

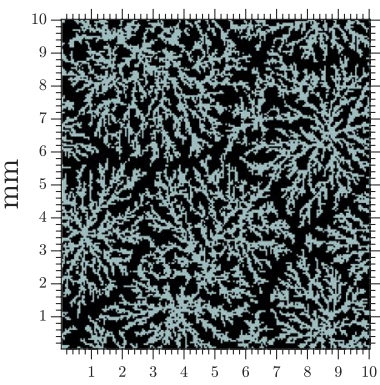

$\mathrm{mm}$

Figure 5. Snapshots of aggregation at different elapsed times. cAMP concentration on the left and cell distribution on the right. Same $\gamma$ scale as in Fig. 3, gray squares show where cells are present and empty spaces are shown in black. Density $=4 \cdot 10^{5}$ cells $/ \mathrm{cm}^{2}(0.4$ mono-layer $)$ and $k_{e}=5.7 \mathrm{~min}^{-1}$. (a) $t=30 \mathrm{~min},(\mathbf{b}) t=75 \mathrm{~min}$, (c) $t=100 \mathrm{~min}$, and (d) $t=150 \mathrm{~min}$.

a)

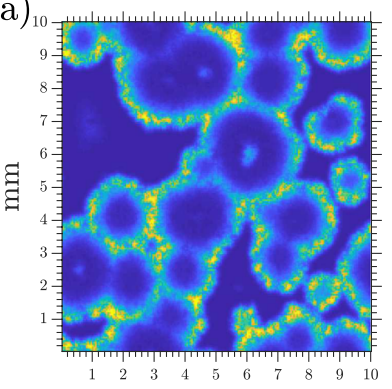

$\mathrm{mm}$

c)

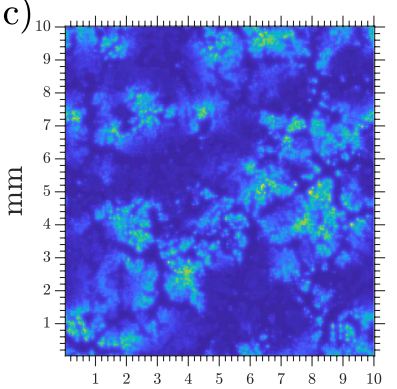

$\mathrm{mm}$

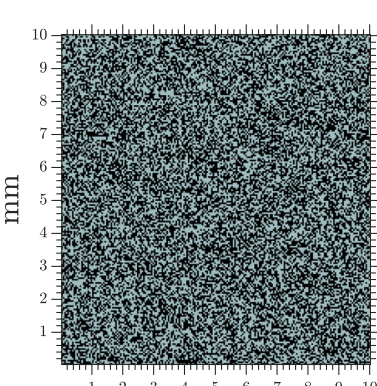

$\mathrm{mm}$

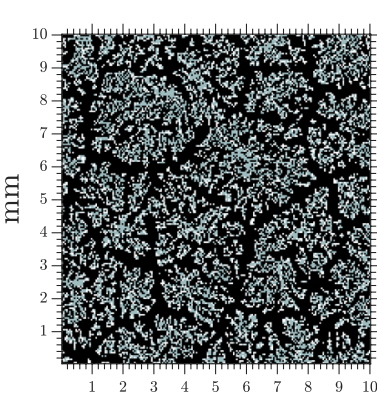

$\mathrm{mm}$ b) $)_{10}$

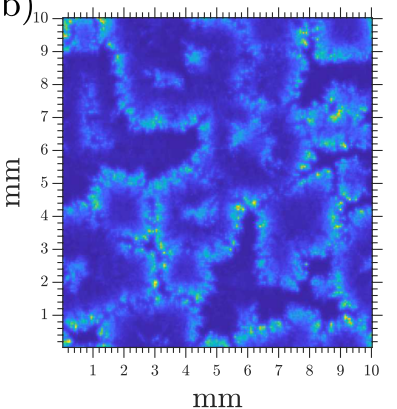

d) $)_{10}$

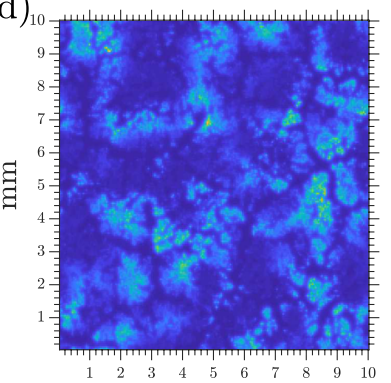

$\mathrm{mm}$

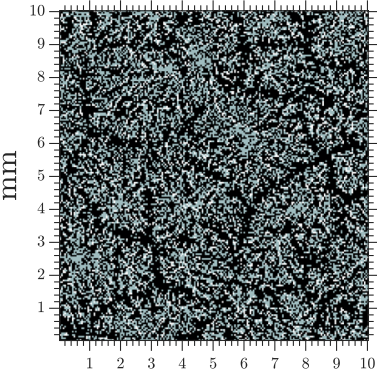

$\mathrm{mm}$

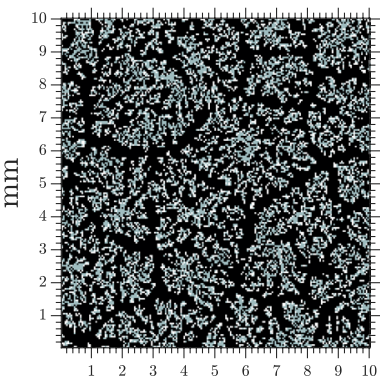

$\mathrm{mm}$

Figure 6. Snapshots of aggregation at different elapsed times with cell superposition, but only unbounded degradation. cAMP concentration on the left and cell distribution on the right. Same $\gamma$ scale as in Fig. 3, gray squares show where one cell is present, white squares contain more than one cell, and empty spaces are shown in black. Density $=5 \cdot 10^{5} \mathrm{cells} / \mathrm{cm}^{2}(0.5$ mono-layer $)$ and $k_{e}=5.7 \mathrm{~min}^{-1}$. (a) $t=39 \mathrm{~min}$, (b) $t=75 \mathrm{~min}$, (c) $t=120 \mathrm{~min}$, and (d) $t=150 \mathrm{~min}$.

producing centers which persist after movement (see Supplementary Video S7). Therefore, we conducted simulations where there is a constant degradation present on the system $k_{e U}$ which represents the unbounded, free phosphodiesterase; and a bounded degradation $k_{e B}$ which exists attached to the outside of the cell membrane and 

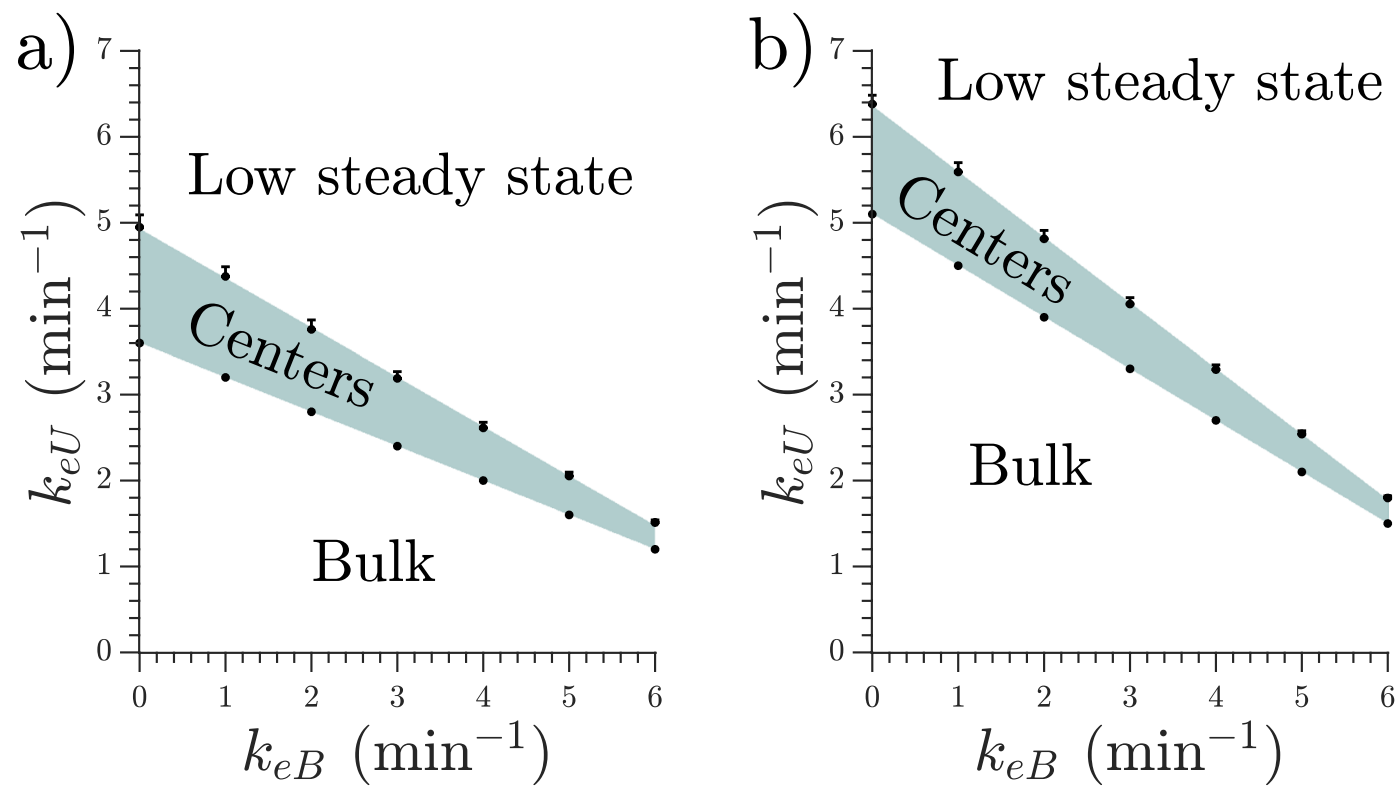

Figure 7. Phosphodiesterase degradation rates at which spontaneous centers exist. Below the shaded area the system presents bulk oscillations. Above the shaded area no spontaneous centers appear. Cell density is $4 \cdot 10^{5}$ cells $/ \mathrm{cm}^{2}\left(0.4\right.$ mono-layer) in panel (a) and $6 \cdot 10^{5} \mathrm{cells} / \mathrm{cm}^{2}(0.6$ mono-layer $)$ in panel (b). Upper boundaries indicate the degradation rate at which $75 \%$ of the simulations presented spontaneous centers. Error bar extends up to $50 \%$. Lower boundary marks the maximum degradation rate at which the homogeneous system presents bulk oscillations.

therefore exists only on the occupied spaces and in an amount dependent on the number of cells in that location. The new equations are then

$$
\begin{gathered}
k_{1}^{-1} \partial_{t} \rho_{i}=-f_{1}\left(\gamma\left(x_{i}, y_{i}\right)\right) \rho_{i}+f_{2}\left(\gamma\left(x_{i}, y_{i}\right)\right)\left(1-\rho_{i}\right), \\
\partial_{t} \beta_{i}=s \Phi\left(\rho_{i}, \gamma\left(x_{i}, y_{i}\right)\right)-\left(k_{i}+k_{t}\right) \beta_{i}, \\
\partial_{t} \gamma(x, y)=D \nabla^{2} \gamma(x, y)-k_{e U} \gamma(x, y)+\sum_{i}^{N} H(i, x, y)\left[k_{t} \beta_{i} / h-k_{e B} \gamma(x, y)\right] .
\end{gathered}
$$

The results obtained with these equations are similar to those shown in Fig. 3, that is, we distinguish three types of behaviour depending on the degradation rates. The degradation combinations for which the system still presents spontaneous centers are shown in Fig. 7. Unlike in the case with no bounded phosphodiesterase, the variance of the normal distribution decreased with the bounded degradation rate, thus making the transition from centers to low steady state much sharper at higher $k_{e B}$.

We see that there is no fixed ratio of bounded/unbounded phosphodiesterase such that the system produces centers, but rather there is a whole range of values, depending on the system density, thus allowing the cells to have some variability and still present the same behaviour. It is noteworthy that as the bounded degradation increases $\left(k_{e B}\right), k_{e U}$ decreases, as does the range of possible unbounded values $k_{e U}$, and the upper transition becomes sharper, since the range of values where $50-75 \%$ of simulations presented centers diminishes (length of the upper errorbars). Thus the cells can have less variability in $k_{e U}$ as $k_{e B}$ increases.

\section{Discussion}

Over the past years several models have been used to describe the patterns observed in D.d. Excitable models are capable of sustaining spiral and trigger waves, but in order to generate a target pattern they require either an oscillatory center or spontaneous/random firing from the cells. On the other hand, oscillatory models require some sort of inhomogeneity to be added to the system to produce stable centers and avoid bulk oscillations. It has been shown that increasing the cell parameters along a developmental path or adding random firing decides the location of the observed patterns in an artificial way ${ }^{25-28}$ which contrast with experimental observations showing that oscillation is a collective effect instead of the work of some specialized cells, where even the cells composing the oscillating center move continuously in and out of the signaling center ${ }^{29,30}$. We suggest that a more simple mechanism is also in play to produce centers for densities less than a mono-layer: the inhomogeneous cell distribution in the system is enough to create stable emitting centers. Our model matches the observation of Lee et al. ${ }^{31}$ who showed that at lower densities (below mono-layer density $\approx 10^{6} \mathrm{cells} / \mathrm{cm}^{2}$ ) target centers dominate the observed patterns, while high above confluency, spirals appear. Our simulations show that below confluency and for a fixed value of $k_{e}$, the number of target centers increases with cell density until the transition to bulk oscillations occur (see Fig. 4a). A recent experiment by Ohta et al. ${ }^{30}$ showed that at densities of the order of $1.25 \cdot 10^{5} \mathrm{cells} / \mathrm{cm}^{2}(0.125$ 

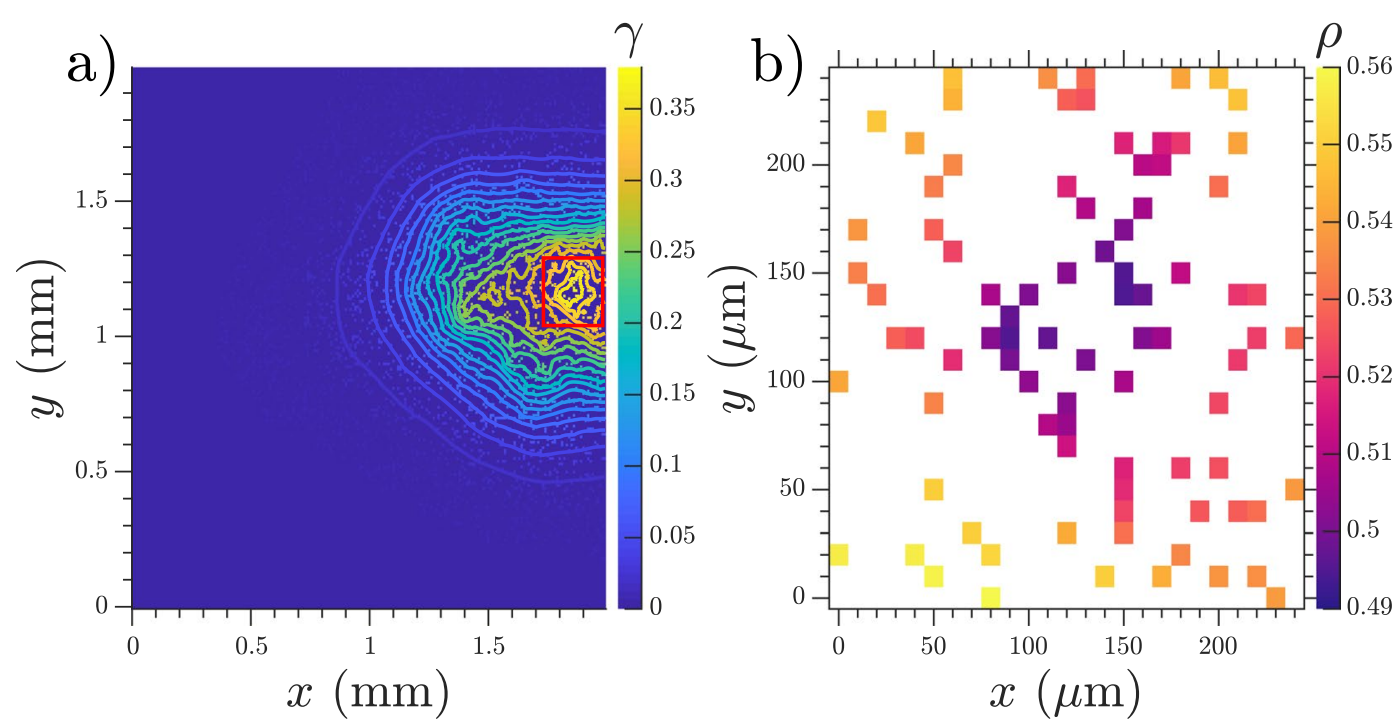

Figure 8. Image of a numerical simulation showing a signaling center with the characteristics of those observed by Ohta et al..$^{30}$. Density $=1.25 \cdot 10^{5}$ cells $/ \mathrm{cm}^{2}(0.125$ mono-layer $), k_{e}=1.56 \mathrm{~min}^{-1}$. (a) cAMP concentration in the areas occupied by cells, with equal concentration levels of the whole extracellular media. (b) Zoom of the signaling center marked with red in (a) showing the percentage of active receptors $\rho$. Cells with lower $\rho$ (dark purple) are those which initially fire. Around 18 cells constitute the firing center.

mono-layer) centers can be generated by groups of roughly 13 synchronized cells in an area of $100 \times 100 \mu \mathrm{m}^{2}$. These results match our observation with $k_{e}=1.56 \mathrm{~min}^{-1}$ where a group of approximately 18 cells produce an oscillatory center, see Fig. 8 and Supplementary Video S8.

At higher cell densities when the pattern evolves towards spiral creation, we expect this mechanism to be less relevant, although our model did show occasionally spirals when the wave front broke due to high inhomogeneities in cell density (see Supplementary Video S9). Above mono-layer densities it has been shown that the cell development inhomogeneity ${ }^{18,32}$ and the strength of the feedback loop on excitability ${ }^{33}$ play a prominent role in the spiral creation process.

We stress the simplicity of this mechanism which does not make big assumptions about the inside state of the cells, but simply requires an external degradation mechanism to be present in the media. Small groups of isolated cells reach a steady state of low cAMP concentration. We showed that this stable solution exist for small clusters, where the system has two solutions, a stable and an unstable one. Once the cluster size increases over a critical value, the cluster is no longer stable and starts to oscillate with a size dependent frequency, therefore bigger clusters have a higher frequency which allows them to dominate over smaller clusters. In simulations with randomized cell locations the centers are not necessarily large clusters of consecutive cells, but rather just areas of a higher local density, where some clusters are usually separated by small distances and act together as a big cluster. Even though this does not exactly match the scenario described in the Oscillatory Clusters section, it does provide an insight into why these centers spontaneously appear. Our model is therefore, a collective effect where groups of cells behave oscillatory and individual cells are excitable to external cAMP pulses, consistent with experimental observations $^{29}$, furthermore the lower cAMP level at lower densities is fundamental for their excitable behavior, displaying an oscillatory behaviour if the cAMP levels are artificially risen ${ }^{29}$.

Having a constant degradation across the entire system is an oversimplification of the real setup where the cells produce different amounts of phosphodiesterase ${ }^{34}$ and it even changes over time ${ }^{35}$, but since the centers exist over a range of degradations (see Fig. 4a) we believe that the observed effect is robust to individual differences of phosphodiesterase production in cells, an effect which is also dampened by phosphodiesterase diffusion. Further modeling including phosphodiesterase diffusion and production is necessary to explore these possibilities.

The target patterns showed to have all the main features of those observed in experiments, like producing trigger waves, having different frequencies, and higher frequency centers taking over lower frequency ones. The system showed a range of degradation values at which it presents centers, this range decreased with increasing density, while the maximum value scaled non linearly with density. For a fixed cell density increasing the degradation rate decreased the average center frequency (see Fig. 4c), consistent with measurements in flow chamber experiments ${ }^{29}$.

Once cell movement was introduced, the system showed streaming when aggregating. We attribute this behaviour to the velocity dependence on density (see Fig. $4 \mathrm{~b}$ ), as it has been shown before in similar descriptions ${ }^{16,17,36}$. In those systems, as in ours, the cAMP wave travels faster on the areas with higher cell density thus producing a wave which is not perfectly circular. This shape leads to an aggregation which is not radially uniform, thus producing streams. In this model the velocity dependence on density comes from the wave speed difference between spaces with cells, where new cAMP is produced, and empty spaces where speed is given only by diffusion, thus on average higher density areas relay waves faster than lower density ones, producing streaming. This simple aggregation model shows that intracellular localization of cAMP or ACA is not necessary to explain 
streams, along with other mechanisms such as cell-cell adhesion, chemotactical memory ${ }^{37}$, and directional rectification $^{38}$, that, although are present in the experimental system, do not seem to be fundamentally necessary for stream formation.

The final section highlights the role of a cell bounded form of degradation. This comes into relevance once the cells have started to move and form small clumps. In order for these clumps to not disrupt the wave propagation process, a degradation that scales with density is necessary. The existence of this type of phosphodiesterase is still a matter of discussion in the experimental community ${ }^{24,34,39,40}$ and even though we believe our model provides arguments in favor of the existence of both types, the effect of locally increasing degradation can also be explained by a local accumulation of phosphodiesterase around the cells possibly due to the recent release of PDE to the extracellular media that has not yet diffused sufficiently.

In conclusion, we have presented a scenario of creation of target patterns in a model describing pattern formation in D.d. This scenario does not require specific stages along the cell developmental path, nor requires the introduction of random firing, both of which preselect the location of target centers. Instead, in this description wave centers appear spontaneously in areas of higher cell density. We have shown that introducing cell inhomogeneity in the Martiel-Goldbeter model creates naturally target patterns that are stable and capable of producing waves that fill the whole system. We characterized these centers, which correspond to areas of higher cell density. The size of the minimum cluster required to produce a center increases with the degradation rate of the system. If the cluster size is not big enough to sustain autonomous oscillations, it reaches a low steady state whose amplitude decreases with the degradation rate. These smaller clusters, or areas of lower local density can nevertheless be excited, thus allowing waves to propagate through the system. Therefore, this scenario reproduces the large scale organization displayed by $D . d$. populations.

\section{Methods}

The system was simulated using a fourth-order Runge-Kutta scheme with Merson correction ${ }^{41}$ and an adaptive time step. We used finite differences and a 5 points laplacian in 2-D and 3 points in 1-D. For 1-D simulations $d x$ was selected equal to the cell size $r=10 \mu \mathrm{m}$. To speed up calculations for most 2-D simulations, $d x=5 r$ was selected, with the results being confirmed by smaller grid sizes.

To calculate the upper boundary of Figs 4a and 7 we simulated the system a hundred times for each set of parameters and recorded how many simulations presented centers. We then fitted a gaussian distribution around the boundary and from this fitting extracted the expected value for a $75 \%$ of center appearance (marked as a circle) and for a 50\%, marked as the end of the errorbar. In Fig. 4, where only unbounded phosphiesterase was present, the fitted gaussian distributions presented the same variance, differing only in their mean. In Fig. 7 the variance lowered with increasing $k_{e B}$.

To calculate the lower boundary we used a extreme case consisting in homogeneously distributed simulations, where cells had a constant distance between them. This is the most spread out possible distribution for the cells, thus avoiding clusters as much as possible. For example, if the density is $5 \cdot 10^{5} \mathrm{cells} / \mathrm{cm}^{2}(0.5$ mono-layer $)$ the distribution would be one cell, then $0.01 \mathrm{~mm}$ of empty space, then another cell and so on. In this homogeneous system if the degradation is too small the cells show bulk oscillations. As we increased the amount of phosphodiesterase the bulk oscillations ceased and the system reached a stable steady state of low cAMP concentration. The lower boundary of Figs $4 \mathrm{a}$ and 7 indicates the maximum amount of phosphodiesterase such that the homogeneous system shows bulk oscillations, therefore at that degradation rate or higher any inhomogeneous cell distribution will produce target centers.

\section{Data Availability}

All data used in this study will be made available upon request.

\section{References}

1. Kessin, R. H. Dictyostelium: evolution, cell biology, and the development of multicellularity, vol. 38 (Cambridge University Press, 2001).

2. Allessie, M. A., Bonke, F. I. \& Schopman, F. J. Circus movement in rabbit atrial muscle as a mechanism of tachycardia. Circ. research 33, 54-62, https://doi.org/10.1161/01.RES.33.1.54 (1973).

3. Lechleiter, J., Girard, S., Peralta, E. \& Clapham, D. Spiral calcium wave propagation and annihilation in xenopus laevis oocytes. Sci. 252, 123-126, http://science.sciencemag.org/content/252/5002/123, https://doi.org/10.1126/science.2011747 (1991).

4. Mair, T. \& Müller, S. C. Traveling nadh and proton waves during oscillatory glycolysis in vitro. J. Biol. Chem. 271, 627-630 (1996).

5. Winfree, A. T. Spiral waves of chemical activity. Sci. 175, 634-636, https://doi.org/10.1126/science.175.4022.634 (1972).

6. Zaikin, A. \& Zhabotinsky, A. Concentration wave propagation in two-dimensional liquid-phase self-oscillating system. Nat. 225, 535-537, https://doi.org/10.1038/225535b0 (1970).

7. Kopell, N. \& Howard, L. Plane wave solutions to reaction-diffusion equations. Stud. Appl. Math. 52, 291-328, https://doi. org/10.1002/sapm1973524291 (1973).

8. Stich, M. \& Mikhailov, A. S. Complex pacemakers and wave sinks in heterogeneous oscillatory chemical systems. Zeitschrift für Physikalische Chemie 216, 521, https://doi.org/10.1524/zpch.2002.216.4.521 (2002).

9. Keener, J. P. A geometrical theory for spiral waves in excitable media. SIAM J. on Appl. Math. 46, 1039-1056, https://doi. org/10.1137/0146062 (1986).

10. Tyson, J. J. \& Keener, J. P. Singular perturbation theory of traveling waves in excitable media (a review). Phys. D: Nonlinear Phenom. 32, 327-361, https://doi.org/10.1016/0167-2789(88)90062-0 (1988).

11. Levine, H., Aranson, I., Tsimring, L. \& Truong, T. V. Positive genetic feedback governs cAMP spiral wave formation in Dictyostelium. Proc. Natl. Acad. Sci. 93, 6382-6386 http://www.pnas.org/content/93/13/6382.abstract http://www.pnas.org/content/93/13/6382. full.pdf (1996)

12. Noorbakhsh, J., Schwab, D. J., Sgro, A. E., Gregor, T. \& Mehta, P. Modeling oscillations and spiral waves in dictyostelium populations. Phys. Rev. E 91, 062711, https://doi.org/10.1103/PhysRevE.91.062711 (2015).

13. Martiel, J.-L. \& Goldbeter, A. A model based on receptor desensitization for cyclic AMP signaling in Dictyostelium cells. Biophys. J. 52, 807, https://doi.org/10.1016/S0006-3495(87)83275-7 (1987). 
14. Tyson, J. J., Alexander, K. A., Manoranjan, V. \& Murray, J. Spiral waves of cyclic AMP in a model of slime mold aggregation. Phys. D: Nonlinear Phenom. 34, 193-207, https://doi.org/10.1016/0167-2789(89)90234-0 (1989).

15. Aliev, R. R. \& Biktashev, V. N. Dynamics of the oscillation phase distribution in the bz reaction. The J. Phys. Chem. 98, 9676-9681, https://doi.org/10.1021/j100089a049 (1994).

16. Höfer, T. \& Maini, P. K. Streaming instability of slime mold amoebae: An analytical model. Phys. Rev. E 56, 2074, https://doi. org/10.1103/PhysRevE.56.2074 (1997).

17. Van Oss, C., Panfilov, A. V., Hogeweg, P., Siegert, F. \& Weijer, C. J. Spatial pattern formation during aggregation of the slime mould dictyostelium discoideum. J. theoretical biology 181, 203-213, https://doi.org/10.1006/jtbi.1996.0126 (1996).

18. Lauzeral, J., Halloy, J. \& Goldbeter, A. Desynchronization of cells on the developmental path triggers the formation of spiral waves of cAMP during Dictyostelium aggregation. Proc. Natl. Acad. Sci. 94, 9153-9158 http://www.pnas.org/content/94/17/9153.abstract, https://doi.org/10.1073/pnas.94.17.9153 (1997).

19. Vidal-Henriquez, E., Zykov, V., Bodenschatz, E. \& Gholami, A. Convective instability and boundary driven oscillations in a reactiondiffusion-advection model. Chaos: An Interdiscip. J. Nonlinear Sci. 27, 103110, https://doi.org/10.1063/1.4986153 (2017).

20. Durston, A. Pacemaker activity during aggregation in dictyostelium discoideum. Dev. biology 37, 225-235, https://doi. org/10.1016/0012-1606(74)90144-4 (1974).

21. Wessels, D., Murray, J. \& Soll, D. R. Behavior of dictyostelium amoebae is regulated primarily by the temporal dynamic of the natural camp wave. Cell motility cytoskeleton 23, 145-156, https://doi.org/10.1002/cm.970230207 (1992).

22. Goldstein, R. E. Traveling-wave chemotaxis. Phys. review letters 77, 775, https://doi.org/10.1103/PhysRevLett.77.775 (1996).

23. Shapiro, R. I., Franke, J., Luna, E. J. \& Kessin, R. H. A comparison of the membrane-bound and extracellular cyclic amp phosphodiesterases of dictyostelium discoideum. Biochimica et Biophys. Acta (BBA)-General Subj. 758, 49-57, https://doi. org/10.1016/0304-4165(83)90009-0 (1983).

24. Bader, S., Kortholt, A. \& Van Haastert, P. J. Seven dictyostelium discoideum phosphodiesterases degrade three pools of camp and cgmp. Biochem. J. 402, 153-161, https://doi.org/10.1042/BJ20061153 (2007).

25. Geberth, D. \& Hütt, M.-T. Predicting spiral wave patterns from cell properties in a model of biological self-organization. Phys. Rev. E 78, 031917, https://doi.org/10.1103/PhysRevE.78.031917 (2008).

26. Geberth, D. \& Hütt, M.-T. Predicting the distribution of spiral waves from cell properties in a developmental-path model of dictyostelium pattern formation. PLoS computational biology 5, e1000422, https://doi.org/10.1371/journal.pcbi.1000422 (2009).

27. Grace, M. \& Hütt, M.-T. Predictability of spatio-temporal patterns in a lattice of coupled fitzhugh-nagumo oscillators. J. The Royal Soc. Interface 10, 20121016, https://doi.org/10.1098/rsif.2012.1016 (2013).

28. Grace, M. \& Hütt, M.-T. Regulation of spatiotemporal patterns by biological variability: general principles and applications to dictyostelium discoideum. PLoS computational biology 11, e1004367, https://doi.org/10.1371/journal.pcbi.1004367 (2015).

29. Gregor, T., Fujimoto, K., Masaki, N. \& Sawai, S. The onset of collective behavior in social amoebae. Sci. 328, 1021-1025, https://doi. org/10.1126/science.1183415 (2010).

30. Ohta, Y., Furuta, T., Nagai, T. \& Horikawa, K. Red fluorescent camp indicator with increased affinity and expanded dynamic range. Sci. reports 8, 1866, https://doi.org/10.1038/s41598-018-20251-1 (2018).

31. Lee, K. J., Cox, E. C. \& Goldstein, R. E. Competing patterns of signaling activity in dictyostelium discoideum. Phys. review letters 76 1174, https://doi.org/10.1103/PhysRevLett.76.1174 (1996).

32. Pálsson, E. \& Cox, E. C. Origin and evolution of circular waves and spirals in dictyostelium discoideum territories. Proc. Natl. Acad. Sci. 93, 1151-1155, https://doi.org/10.1073/pnas.93.3.1151 (1996).

33. Sawai, S., Thomason, P. A. \& Cox, E. C. An autoregulatory circuit for long-range self-organization in dictyostelium cell populations. Nat. 433, 323, https://doi.org/10.1038/nature03228 (2005).

34. Hall, A. L., Franke, J., Faure, M. \& Kessin, R. H. The role of the cyclic nucleotide phosphodiesterase of Dictyostelium discoideum during growth, aggregation, and morphogenesis: overexpression and localization studies with the separate promoters of the pde. Dev. biology 157, 73-84, https://doi.org/10.1006/dbio.1993.1113 (1993).

35. Malchow, D., Nägele, B., Schwarz, H. \& Gerisch, G. Membrane-bound cyclic AMP phosphodiesterase in chemotactically responding cells of Dictyostelium discoideum. Eur. J. Biochem. 28, 136-142, https://doi.org/10.1111/j.1432-1033.1972.tb01894.x (1972).

36. Vasiev, B., Hogeweg, P. \& Panfilov, A. Simulation of dictyostelium discoideum aggregation via reaction-diffusion model. Phys. Rev. Lett. 73, 3173, https://doi.org/10.1103/PhysRevLett.73.3173 (1994).

37. Skoge, M. et al. Cellular memory in eukaryotic chemotaxis. Proc. Natl. Acad. Sci. 111, 14448-14453, https://doi.org/10.1073/ pnas.1412197111 (2014).

38. Nakajima, A., Ishihara, S., Imoto, D. \& Sawai, S. Rectified directional sensing in long-range cell migration. Nat. communications $\mathbf{5}$, 5367, https://doi.org/10.1038/ncomms6367 (2014).

39. Garcia, G. L., Rericha, E. C., Heger, C. D., Goldsmith, P. K. \& Parent, C. A. The group migration of dictyostelium cells is regulated by extracellular chemoattractant degradation. Mol. biology cell 20, 3295-3304, https://doi.org/10.1091/mbc.e09-03-0223 (2009).

40. Xiao, Z. \& Devreotes, P. N. Identification of detergent-resistant plasma membrane microdomains in dictyostelium: enrichment of signal transduction proteins. Mol. biology cell 8, 855-869, https://doi.org/10.1091/mbc.8.5.855 (1997).

41. Merson, R. An operational method for the study of integration processes. In Proc. Symp. Data Processing, 1-25 (1957).

\section{Acknowledgements}

The authors thank E. Bodenschatz, A. Bae, and V. Zykov for fruitful discussions. E.V.H. thanks the Deutsche Akademische Austauschdienst (DAAD), Research Grants-Doctoral Programs in Germany. A.G. acknowledges MaxSynBio Consortium, which is jointly funded by the Federal Ministry of Education and Research of Germany and the Max Planck Society.

\section{Author Contributions}

E.V.H. and A.G. designed the research, E.V.H. performed the numerical simulations and theoretical analysis, E.V.H. and A.G. wrote and reviewed the manuscript.

\section{Additional Information}

Supplementary information accompanies this paper at https://doi.org/10.1038/s41598-019-40373-4.

Competing Interests: The authors declare no competing interests.

Publisher's note: Springer Nature remains neutral with regard to jurisdictional claims in published maps and institutional affiliations. 
(c) (i) Open Access This article is licensed under a Creative Commons Attribution 4.0 International License, which permits use, sharing, adaptation, distribution and reproduction in any medium or format, as long as you give appropriate credit to the original author(s) and the source, provide a link to the Creative Commons license, and indicate if changes were made. The images or other third party material in this article are included in the article's Creative Commons license, unless indicated otherwise in a credit line to the material. If material is not included in the article's Creative Commons license and your intended use is not permitted by statutory regulation or exceeds the permitted use, you will need to obtain permission directly from the copyright holder. To view a copy of this license, visit http://creativecommons.org/licenses/by/4.0/.

(C) The Author(s) 2019 


\section{Discussion}

During this work the theoretical framework of the Martiel-Goldbeter model was modified to perturb the pattern formation process in D. discoideum in two different ways: due to advection and due to obstacles. In both cases numerical and theoretical results were presented, the limits and predictions of the model were tested, along with qualitative comparisons with experimental results.

\subsection{Perturbation by Advecting Flows}

When an advecting flow is applied to a colony of $D$. discoideum a new dynamical regime appears [86]. In this convectively unstable regime the steady state solution destabilizes when a flow above a critical velocity is applied. This instability could be particularly relevant in the natural habitat of the amoebas, where the cells are constantly under advecting flows on the soil of forests. In this regime, a perturbation produces a growing wave train in which three relevant speeds were identified and calculated: the speed of the back of the wave train $v_{b}$, the speed of the front of the wave train $v_{f}$, and the individual peak velocity $v_{p}$ (see Figure 3 in Chapter 2 for a graphical representation of these velocities in the wave train). The front and back of the wave train velocities were calculated using the steepest descend method to obtain the long term behavior of the system in a moving reference frame [111]. They correspond to the minimum and maximum velocities such that, if the reference frame is moving at that velocity, the perturbation has a positive growth. The front velocity $v_{f}$ is always higher than the advecting flow velocity $V$, while the back velocity $v_{b}$ is always lower than the imposed one. The predicted velocities through this linear analysis matched excellently with the velocities measured in the full nonlinear simulation.

Since the velocity of the front and back of the wave train are different, the wave train grows in size as it is advected downstream, producing a train with an increasing amount of peaks as it travels. Following a particular peak or valley inside the wave train shows that the peaks in the middle of the wave train move faster than in the front of the wave train, therefore the peaks change their relative location in the wave train, moving towards the front, and then slowing down when they reach the front of the train 
to match the front of the train velocity $v_{f}$. In other words, at a fixed point in time the peaks inside the wave train have different instant velocities depending on their position inside the wave train, lowest at the back $\left(v_{b}\right)$, rapidly increasing at the middle up to $v_{p}$, and then decreasing towards the front to match $v_{f}$. Since period and velocity are uniquely determined by the dispersion relation of the system, the wavelength is smaller at the front of the train than in the middle of it, producing a peak accumulation at the front of the wave train. This traffic jam of wave peaks is known as wave stacking and it has been reported in other excitable systems $[112,113]$.

The convectively unstable regime exists at lower degradation rates than the oscillatory regime and thus provides a mechanism for wave creation in phosphodiesterase deficient systems. For example, setups with very low cell densities, cells in early stages of development at the onset of starvation, $p d s A^{-}$cells, among others. In such a system it is of utmost importance what kind of chemicals gets injected in the system with the advecting flow. In the numerical simulations this is equivalent to the boundary conditions used in the upstream edge of the system. In the case where there are no cells upstream the flow would be free of cAMP and can act as a destabilizing agent.

It was shown that holding the upstream boundary to a zero concentration of cAMP produces an instability that periodically sends wave trains downstream. The wave generation mechanism works by first advecting downstream the cAMP, thus depleting the upstream area of cAMP, this low concentration of the chemoattractant destabilizes the cells close to the upstream boundary, which react by releasing a pulse of cAMP. This pulse gets advected downstream and produces a wave train as previously described, but it also excites the cells upstream from the destabilization point. This excitation produces a pulse that travels upstream until it reaches the boundary, where it is absorbed and the cycle starts again. The period of this cycle, and therefore the period with which a new wave train is advected downstream, is given by how long it takes for the pulse to travel up to the upstream boundary. The velocity of this pulse depends on the advecting flow velocity, and thus with faster advecting flows the period of oscillation is longer. The nucleation point for this instability also depends on the advecting flow and it is located farther away from the upstream boundary when faster flows are applied (see the plots in Figure 10 in Chapter 2 for these dependencies on velocity). At higher velocities of the advecting flows the upstream traveling pulse can not propagate and the system reaches a time independent steady state instead of showing waves.

A situation such as this can be recreated in a laboratory setting by advecting fresh buffer in a microfluidic channel where cells are located at the bottom. Numerical simulations of such a microfluidic setup in 2-D show a very particular shape of the upstream traveling pulse. Due to the small height of this setup, the boundary layer is very small $(\approx 50 \mu \mathrm{m})$ and the advecting flow has mostly a planar profile in the top view projection 
(see Figure 3.1 at the end of Chapter 3). Even when this advecting flow is mostly planar the upstream traveling pulse acquires a cusp in the middle as it travels up the channel, reaching a triangular shape.

This kind of wave deformation when traveling against a Poiseuille flow has been previously described for fronts in auto-catalytic reactions [114, 115], where only one component is involved in the chemical reaction. It is remarkable that a similar shape is achieved in a system where two components are involved, one of which does not move with the advecting flow (the percentage of active receptors $\rho$ ). Therefore, the triangular shape is achieved even though the slow reacting variable does not move with the triangular shape. Other example of a pulse acquiring a triangular shape when traveling against a Poiseuille flow was presented by Ermakova et al. [116] where the FitzHughNagumo model with advection was used. In that case both reactants were advected and could diffuse. Another key difference between these auto-catalytic or excitable systems and the Martiel-Goldbeter model here studied is that the parameter regime in which the Martiel-Goldbeter system was studied does not normally allow the creation of single pulses. That is, under these parameters (in the convectively unstable regime) an external perturbation in the cAMP concentration produces wave trains traveling downstream, not pulses. This is different from the FitzHugh-Nagumo model which was studied in the excitable regime, where the stable solution is the emergence of traveling pulses. The pulses traveling upstream observed in the convectively stable regime have the characteristics of a trigger wave, typical for the excitable regime of this model. This is an example of this system behaving like an excitable system when the cAMP concentration is kept to a low value through external effects. In this case is the absorbing boundary condition that together with the advecting flow keep the cAMP concentration low in the area close to the upstream boundary. This idea of the system behaving as excitable under such conditions appears also later in our study of the effects of a discrete cell distribution in this model.

Unfortunately we have no evidence of experimental observations in wild type cells of this wave creation instability. This discrepancy seems to come from the states (parameters such as degradation and production rate) the cells undergo during their development, where wild type cells do not seem to cross the parameter range where this boundary driven oscillations exists. An interesting approach for experimental confirmation is provided by $p d s A^{-}$cells, which do not produce phosphodiesterase, although they produce cAMP, and therefore do not produce cAMP waves naturally. A flow of clean buffer might provide the destabilizing mechanism necessary for wave production, thus showing boundary driven oscillations. Experiments and numerical studies are currently exploring these possibilities.

The second work presented in this thesis [76] focuses on the effects of high speed 
flows on $D$. discoideum pattern formation. In its natural habitat $D$. discoideum can be subjected to flows up to the order of $100 \mathrm{~mm} / \mathrm{min}$ [117] which can have an enormous impact in cAMP signaling and consequently in Dictyostelium's survival. As part of this work the shape of the wave front in the 2-Component description was compared with the 3-Component version of the Martiel-Goldbeter model, and striking differences were found. When a Poiseuille flow was applied, the shape acquired by the fast reaction model (2-Component) was very similar to the applied flow shape, and, even more, was almost identical to the shape acquired by non-reacting particles being only advected by flow. Therefore, the wave shape of the fast reacting cAMP wave was mostly given by the flow profile, and not by the internal chemical reactions. The opposite case is true for the 3-Component model, where even though a mostly planar profile was applied, the wave had a strong parabolic shape (see Figure 6 in Chapter 3). The wave profile showed by the 3 -Component model was much more similar to the waves observed in our experiments, thus highlighting the importance of slow internal cAMP build-up inside the cell before its release to the external media.

Because of its fast reaction scheme, the 2-Component model presented a very sharp wave front, which means that the transition between areas without cAMP and areas with high cAMP concentration was very abrupt. In contrast, for the 3-Component model this transition was smoother, with a slower build-up of the chemical before reaching its maximum concentration. As a consequence of this, the 3-Component model shows wider waves than its 2-Component counterpart. Both schemes presented an almost linear increase in wave width with increasing advecting velocity, therefore at higher flow speeds the waves were wider. This correlated well with our experimental observations where the width also scaled linearly with the imposed flow. Furthermore, the wave width dependence on flow seemed to be almost instantaneous, as the experiments when the flow was stopped showed. In those experiments the wave profile changed immediately when the applied flow changed speed, therefore showing characteristics of a trigger wave. This reaction to the applied flow was successfully reproduced by our numerical simulations (see, for example, Figure 14 in Chapter 3). Increasing width with flow has also been reported in the FitzHugh-Nagumo model with advection [116] and seems to be a generic characteristic of excitable systems, although the particular way in which the width scales with speeds depends on the specific system. This phenomenon can be understood by noticing that since the flow advects the activator, it is capable of exciting areas of the system that are located farther away in a shorter time than diffusion would do alone and thus producing a bigger area where the system is excited simultaneously, and therefore a wider pulse.

At higher flow speeds the wave profile becomes extremely elongated in the flow direction, with the front of the wave quickly traveling the whole length of the channel, 
while the sides of the parabolic profile move slowly across the channel's shorter length. The wave velocity perpendicular to the flow was measured to be constant and consistent with the wave velocity in the absence of flow (approximately $0.4 \mathrm{~mm} / \mathrm{min}[118]$ ). Since the leading front of the wave profile leaves the channel so quickly at high flow speeds, the observed wave usually looks like a pulse wall almost parallel to the channel's long axis which moves towards the upper or lower boundaries (see Figure 9 in Chapter 3). Because of this, the wave initiation point is crucial to the pattern formation, that is, where the wave originates along the vertical axis determines the shape of the flow profile. The wave shapes shown by the experimental setup were successfully reproduced by our numerical simulations, as can be seen by comparing Figures 5 and 9 in Chapter 3 .

To produce waves in this numerical setup we chose the developmental path approach, originally described by Lauzeral et al. [78] and explained in Section 1.3. In this developmental scheme we observed that the waves originate when a sufficiently big group of cells located in the upstream boundary is in the oscillatory regime. This is in accordance with the observations by Geberth and Hütt [82] who also observed that the cell groups more advanced on the developmental path become the first target centers. It is, however, different in our setup that this oscillating cluster needs to be located upstream if the flow velocities are big. That means, that the waves are only initiated upstream in the channel, with oscillatory clusters downstream failing to produce a traveling wave, which is in agreement with our experimental observations. Both in experiments and in simulations, waves can be started along the entire length of the channel if the imposed flow is slow enough. The explanation for the lack of wave creation along the channel is not yet clear, but we think it has to do with the quick advection of cAMP when it is produced by the oscillatory cells downstream, therefore cAMP does not accumulate fast enough to excite neighboring cells, since the fast flow quickly advects small quantities.

The period of the waves was shown to be independent of imposed flow velocity, both in experiments and simulations. This provides a timescale for cell aggregation which is robust to different flow velocities, thus ensuring aggregation and therefore survival in these very extreme conditions. In regards of this, in our experiments we observed aggregation for all the tested flow speeds, which were up to $50 \mathrm{~mm} / \mathrm{min}$. The aggregation patterns were different than in the absence of flow, since in most cases they did not present streaming. The lack of streaming is expected under the assumption that wave velocity needs to scale with density in order to produce streams; since in the microfluidic channel setup wave velocity depends mainly on imposed flow, the lack of stream is not surprising. The aggregation patterns observed were mostly round clusters of cells that follow a triangle-like distribution with the angle opening towards downstream (see Figure 16 in Chapter 3).

Due to its excellent correlation with experimental observations we concluded that the 
developmental path scheme can successfully be applied to study the effects of advecting flows in D. discoideum. On the other hand, the 2-Component version of the MartielGoldbeter showed to be incapable of reproducing the experimentally observed features, highlighting the importance of the slow build-up of cAMP concentration in the amoebas' intracellular media.

Since advecting flows are ubiquitous in nature, the results here presented might be useful for other excitable or oscillatory systems subjected to advection. The velocity and shape of the applied flows and particularly the boundary conditions upstream play a crucial role in the characteristics of the chemical waves. Here it was shown how in this system they can lead to new instabilities and provide a new wave creation mechanism, produced by the absorbing boundary condition. It was also shown that the triangular deformation of waves traveling upstream can also occur in systems when only the activator is being advected while the other reactants remain stationary, thus increasing the number of systems where this phenomenon could potentially be observed. And in particular for this system we have shown how the signaling mechanism developed by $D$. discoideum is robust enough to still allow for aggregation under extremely stressful environmental conditions, thus ensuring the organisms' survival.

\subsection{Perturbation by Obstacles and Spontaneous Target Centers}

The experiments presented in the third work in this thesis, Chapter 4, show that aggregation of $D$. discoideum can be controlled using pillars as centers of aggregation. In our experimental setup a colony of $D$. discoideum is placed on a PDMS base with pillars protruding from it, also made from PDMS. These pillars have a diameter of $1 \mathrm{~mm}$, and are thus much bigger than the average cell size of $10 \mu \mathrm{m}$. Once signaling starts, concentric circular waves propagate from the pillars, these waves annihilate and interact as normal target waves, with higher frequency centers taking over lower frequency ones. Of vital importance for the experiments to work is the addition of caffeine to the buffer during both the starvation and the signaling part of the experiment. Using numerical simulations we were able to reproduce the ordered aggregation patterns forming squares and hexagons, along with successfully reproducing the effects of caffeine, and shed some light on what chemical mechanisms might be in effect to produce these patterns.

It has been shown that caffeine is a cAMP suppressor [34] that at small concentrations still allows for normal signaling and aggregation. In our setup we added $2 \mathrm{mM}$ of caffeine which increases the oscillation periods from 5-6 min to 9-10 min [36], and diminishes the number of centers appearing [119], thus leading to larger aggregation areas (see Supplementary Figures in Chapter 4).

To account for the addition of caffeine we modified the parameter that accounts for 
the affinity of the ATP for the ACA, that is, we increased the Michaelis constant of the reaction that produces cAMP. This modification to the model is capable of reproducing many of the effects observed in our experiments: the period is increased in a dose-dependent manner and the amount of centers is decreased. To study these properties we used the cellular-automata like model described in Chapter 5 and measured the behaviour of the trigger waves as the amount of caffeine was increased (i.e. the parameter $K_{m}$ was increased). We found that increasing the caffeine concentration reduces wave speed and increases wave period, thus reproducing previous experimental observations [36].

Our numerical simulations also reproduce the decrease in the number of aggregating centers in the presence of caffeine. This decrease is explained by the minimum size a cell cluster needs to have in order to become an oscillating center. In this model, areas of high cell density are capable of oscillating autonomously while lower density areas relay the trigger waves. When caffeine is added, the density necessary to produce a center is increased. Therefore, given the same average density, a population with caffeine would produce less aggregating centers than one without caffeine. We think that this decrease in aggregating centers is fundamental to have a clear controlled aggregation, since a large number of centers appearing in random locations away from the pillars would interact and interfere with the waves emanating from the pillars. In our experimental setup adding caffeine also reduces the amount of spirals observed, producing more target waves than spiral ones. Since spiral waves have a higher frequency than target patterns they would take over the waves emanating from pillars and thus impede the control over the final pattern. To further encourage the apparition of centers we also chose a starvation time of 4 hours which was shown by Prabhakara et al. [120] to mainly produce centers. For this reason we chose a numerical scheme that focuses on target pattern appearance over spirals.

Nevertheless, numerical simulations showed that these effects produced by caffeine were not enough to account for the experimental observations. In order for the pillars to act as centers a special boundary condition needed to be applied. If the pillars were considered as an ordinary wall, a no-flux boundary condition would have been the proper numerical representation, but in this case the pillars were just ignored, similar to our experimental observations in the absence of caffeine. Through numerical simulations different boundary conditions and scenarios that would explain trigger waves coming from the pillars observed in experiments were explored.

The first scenario, and the one we deemed as more likely, is a slightly higher cell density around the pillars. This might happen due to cells adhering to the side of the pillar, or accumulating in the corners when the amoebas are poured. Numerical simulations showed that a slight cell accumulation around the obstacles was enough to 
produce outgoing circular waves.

Related to the first scenario is the possibility of cAMP adsorption on the pillars. PDMS is known to be highly permeable to water and capable of adsorbing small molecules [121]. This was numerically described as a fixed boundary condition in the simulations $\left(\gamma=\gamma_{0}\right.$ at the pillar interface). We found that even very small concentrations of cAMP around the pillar $\left(\gamma_{0} \approx 6 \mathrm{nM}\right)$ were capable of producing outgoing waves.

The last possible scenario is the adsorption or depletion of another chemical around the pillars. Numerical simulations showed that a higher concentration of phosphodiesterase around the pillars produces inward traveling waves, while with phosphodiesterase depletion the pillars act as wave sources.

Any of these scenarios should also theoretically work without the presence of caffeine, which means, that even if afterwards spirals or other target centers take over the system, still some waves should be observed emanating from the pillars, at least on the early stages of signaling. This is not the case in our experiments, where in the absence of caffeine no waves centered around the pillars were observed. This means that caffeine is acting in yet another way to control the pattern formation. Numerical simulations showed that caffeine also reduces the threshold necessary for cell excitation, this means that the cells are more sensitive and therefore capable of reacting to smaller concentrations of cAMP. This change in sensitivity is enough so that the cells can react to the cAMP adsorbed in the pillars, or the cAMP produced by the cells accumulated around it.

This difference in sensitivity sheds a new light on how D. discoideum detects cAMP. Previous studies have shown that $D$. discoideum is extremely sensitive to cAMP, but just how sensitive seems to be a key point to survive in the wilderness. If $D$. discoideum were more sensitive to cAMP than it is, the cells would risk aggregation around natural obstacles, as our experiments show, which would prevent the correct development of a mound and then further survival. If $D$. discoideum were less sensitive, then the whole aggregation process could be compromised. This trade-off between avoiding obstacles and having long distance aggregation defines just how sensitive D. discoideum is to cAMP.

The fact that caffeine increases $D$. discoideum sensitivity was briefly discussed by Brenner and Thoms [34] who reported that cells reacted to cAMP concentrations 100 times smaller in the presence of $3 \mathrm{mM}$ of caffeine, but that they showed the same sensitivity at lower densities. They attributed this effect to a decrease in the basal level of cAMP, therefore with a lower external cAMP concentration there would be less competition between the cAMP waves and the external base stimulus, allowing the detection of smaller gradients. It has also been reported that caffeine increases cell adhesion [119] 
and that could have effects on cell motility. But since the ordered signaling patterns are established before the cells start to move against the wave direction, we considered these effects as less relevant for this study.

The exact way in which caffeine affects cAMP production has not been established yet. It has been suggested that there is more than one caffeine target [37], one of which is located downstream the G-protein in the ACA activation path. To test the particular effect of having less activated ACA, we also ran simulations modifying the parameter $\epsilon$, which is proportional to the ratio of activated ACA to not activated ACA. The simulations showed similar results to those obtained modifying $K_{m}$, that is, decrease in the number of centers and lower activation threshold for relaying signals. Therefore, regardless of the particular way in which caffeine affects ACA our numerical results are consistent and provide an explanation for the observed behavior.

Controlling the patterns shown by reaction-diffusion systems has been previously achieved in chemical reactions such as the light sensitive Belousov-Zhabotinsky [122], where the pinning of spirals around non excitable obstacles has been observed. The interaction between spirals and obstacles has been numerically and experimentally studied $[123,124]$. Furthermore, it has also been shown that the pinned spirals can be unpinned applying an electric field $[125,126]$. Nevertheless, control over the aggregation patterns of $D$. discoideum has not been previously achieved. Our experiments open new possibilities to aggregate chemotactically competent cells and provide a useful test playground for the predictions and properties of the wave propagation models used to describe them.

Since part of the description of this system involved cell aggregation we modified the Martiel-Goldbeter model to account for cell density, with the objective of producing mostly target patterns, instead of spiral waves. Additionally, to study cell streaming, a discrete cell distribution was chosen, over a continuous description. An early attempt to unify discrete cells with the Martiel-Goldbeter model was done by van Oss et al. [106] who were unable to obtain cell streaming using the 3-Component description of the model, but observed streaming with the 2-Component model. They attributed this difference to the fact that in one of the models (2-Component) the wave velocity depended on cell density, while in the other it was mostly constant, and thus independent of density. Given the fact that the 2-Component model showed strong deficiencies at reproducing correctly the wave fronts under high speed advection flows (see Chapter 3 ), it was important to find a reconciliation between the 3-Component model and streaming behavior. The key to solve this apparent contradiction resides in the existence of unbounded phosphodiesterase. In van Oss description cAMP degradation exists only in the areas were cells are located, which is equivalent to only the kind of phosphodiesterase that is bounded to the cell membrane. We modified this description to account for the phosphodiesterase that is released to the extracellular media, that is, the un- 
bounded phosphodiesterase. This modification was enough to produce a wave speed that was density dependent (see Figure $4 \mathrm{~b}$ ) in Chapter 5), given that degradation is present everywhere, the extra speed-up that the wave receives when a cell releases cAMP is enough to account for the speed difference at higher densities. With this dependence the model is capable of reproducing the distinctive aggregation patterns displayed by D. discoideum.

A careful study of this model showed that small cell clusters reach a time independent steady state, where cells accumulate cAMP around them and it quickly decays exponentially outside the cluster. This steady state solution does not exist for bigger cell clusters, who acquire an oscillatory nature, therefore cell clusters above a critical size oscillate with a size dependent frequency. Bigger clusters oscillate at higher frequencies than smaller clusters. As expected, this critical cluster size increases with parameters or chemicals that reduce the amount of cAMP in the extracellular media, like increasing the degradation rate given by phosphodiesterase, or adding caffeine to the system.

Even though the small cell clusters can not oscillate by themselves, they are capable of relaying cAMP waves, i.e., they act as an excitable system. This happens because the cAMP diffuses from the small clusters to the empty spaces next them, producing a low cAMP stable steady state in the cluster. When the system is forced to maintain a low cAMP concentration (either through flow as in Chapter 2 or due to diffusion as in Chapter 5) the cells have most of their receptors in the active state and are, therefore, very excitable and react quickly to detecting cAMP, behaving as an excitable system.

This combination of oscillating areas of high density and excitable lower density areas allows the centers spontaneously appearing to produce waves that can effectively fill the entire setup, because they are relayed by the lower density areas. When using a random cell distribution the areas of local higher density start to oscillate, producing expanding concentric circular waves and thus becoming centers of aggregation. Adding a simple model of cell movement in conjunction with this model was enough to produce cell streams comparable to those observed in experimental setups.

We showed that given a fixed cell density a range of degradation rates exists such that these spontaneous centers appear (see Figure 4 in Chapter 5). If degradation is higher, the center size necessary to produce a target pattern is too big and therefore very unlikely to occur. On the opposite end, if degradation is too low, too many centers appear which synchronize and the system exhibits bulk oscillations. This phenomenon is observed using only unbounded phosphodiesterase or a mixture of both bounded and unbounded. As previously mentioned, using only bounded phosphodiesterase was ruled out for not producing streams at densities below mono-layer [106].

The amplitude equation of this system in the oscillatory regime was calculated and simulations were conducted using the Complex Ginzburg-Landau (CGL) Equation and 
a similarly discretized system. Such system did not present target patterns and was incapable of reproducing the results obtained with the full Martiel-Goldbeter model, even though the results in 0-D were favorables (see Appendix). We think this discrepancy comes from the lack of excitable behavior in the CGL Equation, which at low amplitude produces small oscillations, in contrast with the Martiel-Goldbeter model which at low concentrations of cAMP produces an excitable response. Therefore, even when the reaction was discretized in cell-like compartments, the CGL did not present trigger waves and could not reproduce the expected behavior. This supports our argument that an excitable regime close to the oscillatory behavior is necessary to observe the spontaneous apparition of centers, but further numerical experiments with the CGL are necessary to fully rule out the existence of trigger waves in this setup.

Once the cells start to move the differences between the contributions of bounded and unbounded phosphodiesterase are highlighted. If only unbounded phosphodiesterase exists then once the cell streams reach very high densities (above confluency) they become themselves centers, producing breakage in the waves and streams, not forming the connected big streams observed in nature. This can be easily fixed by adding some bounded phosphodiesterase, which recovers the stream behaviour. Again a range of degradation rates exists such that this behavior is observed, not relying on a particular combination or ratio between bounded and unbounded degradations.

It is relevant to highlight the simplicity of the movement mechanism, that without including random motion, nor interaction between cells (such as cell-cell adhesion), is capable of reproducing aggregation patterns. In the experimental community different ideas have been proposed to explain the appearance of cell streaming, for example, that ACA is localized in the cells' tails [127, 128], which was later disputed by Mukai et al. who did not find polarized accumulation of ACA using GFP knock-in cells [129]. Our model shows that an intracellular localization of ACA or cAMP is not necessary to produce streaming patterns. Consistently, the lack of aggregation streams observed by Kriebel et al. [127] in the ACA null mutants can be explained by the independence of wave velocity on density, due to the lack of cAMP production by the cells.

This study provides a new mechanism for the formation of target patterns in $D$. discoideum. This mechanism is particularly relevant at cell densities below confluency, where it has been shown that centers dominate over spirals [100]. It is also consistent with experiments that have shown that signaling is a collective effect of a group of cells firing synchronously [130, 131] and not the work of some specialized cells as has been assumed in other models $[84,96]$. In particular, our simulations fit the experiments by Ohta et al. [131] who observed a group of 13 cells firing synchronously to produce a center when the system had a density of $1.25 \cdot 10^{5}$ cells $/ \mathrm{cm}^{2}(0.12$ mono-layer, see Figure 8 in Chapter 5 for a comparison of our simulations and this experiment). Our 
model is capable of producing centers even though it assumes identically developed cells, which would explain the experimental observation that cells can move in and out of the firing centers, without the centers losing their signaling capabilities [130]. Therefore, our model explains the prevalence of target centers at low densities and the experimental observations of the characteristics of such centers, giving a clear explanation to the center apparition phenomenon which is robust to a wide range of parameters and degradation rates. 


\section{$7 \quad$ Conclusions and Outlook}

In this cumulative thesis the Martiel-Goldbeter model was used to describe externally perturbed patterns in $D$. discoideum. The model was studied mostly through numerical simulations whose results were compared with experimental observations.

We have presented a mechanism for wave generation in flow advected environments, which acts at lower degradation rates than the normal oscillatory behavior of the system. This instability occurs when the system is advected with a flow that is cAMP-free, represented by an absorbing upstream boundary condition. Under these conditions the amoebas periodically produce wave trains that are advected downstream and a traveling wave pulse that travels upstream. In a 2-D setup under a Poiseuille flow, this upstream traveling peak acquires a triangular shape, that elongates with increasing advecting speeds. The diverse speeds shown by the downstream traveling wave trains were also characterized.

The predictions of both the fast and slow reacting versions of the Martiel-Goldbeter model were tested under fast advecting flows, with the 3-Component model outperforming the 2-Component one, highlighting the importance of accumulation of intracellular cAMP before its release to the external media. The 3-Component model along with a developmental path scheme correctly predicted the speed and shape of the downstream traveling waves at high speed flows showing excellent agreement with experimental observations. The wave shape was shown to be extremely dependent on the location of the cells firing initially, who can only successfully produce waves if they are located close to the upstream boundary.

The mechanism of center creation at densities below confluency was studied and explained. Areas of locally higher cell density act as autonomous centers while lower local density areas have excitable characteristics and are capable of relaying the waves generated by the centers. Bigger clusters produce higher frequency centers, while higher degradation rates decrease the average frequency of the system. The waves produced through this mechanism are of the traveling wave type and produced streaming lines once cell movement was incorporated into the system. This center behavior is consistent with experimental observations, where it has been shown that at densities below confluency centers dominate the pattern formation, and that these centers consists of 
groups of cells firing synchronously, without them having particular characteristics in their development that distinguish them from other cells.

We also showed that the signaling centers can be controlled by adding caffeine to the system. Under this lower cAMP production condition millimetric pillars act as sources for target centers, therefore effectively controlling the aggregation process in $D$. discoideum. This effect was successfully reproduced in numerical simulations modifying parameters to account for the caffeine effects and adding an appropriate boundary condition at the pillars' boundaries. It was shown that caffeine increases $D$. discoideum sensitivity to cAMP, reduces the amount of aggregation centers, and decreases the period of the system. Either a higher cell accumulation around pillars or chemical adsorption on their surface are necessary to effectively produce signaling centers around the obstacles. The amoebas with increased sensitivity can then react to these effects and aggregate around obstacles.

The majority of the results in this work occur due to the existence of an excitable behavior close in the parameter space to an oscillatory behavior. This characteristic can be found in many other systems, and therefore the predictions here presented can be useful to other dynamical systems subject to advection, or when the reactions occur in discrete independent containers distributed throughout the system.

The success of the Martiel-Goldbeter model in explaining the behavior of signaling in $D$. discoideum under diverse conditions shows that it can be successfully modified to account for different settings and cell growing conditions. The amount of parameters and the detailed derivation from chemical reactions allow the easy separation of the effects of chemicals or different cell strains with knock-out genes. We expect to continue using this model to address other problems in D. discoideum. This problems include, but are not limited to, study numerically the existence of Arnold Tongues in the forced system (such a phenomenon has already been experimentally observed [132]), study the recovery of $p d s A^{-}$cells under advecting flows, numerical studies of the Martiel-Goldbeter Model in 3-D, and quantitative comparisons with other models, such as models with scale invariance [133].

Finally, we expect these results to help elucidate the control of multicellular aggregation which can be useful in the context of biofilms and morphogenesis. 


\section{Appendix: Comparison of Oscillatory Regime to Its Amplitude Equation}

Close to a Hopf-bifurcation the timescales of the system are clearly separated, this allows us to eliminate the fast variables and describe the system through its amplitude equation. The parameters describing the amplitude equation of the Martiel-Goldbeter model can be systematically calculated following the procedure laid out by Ipsen et al. [58]. Rewriting Equation 1.5 in a vector format we obtain

$$
\frac{\partial}{\partial t}\left(\begin{array}{l}
\rho \\
\beta \\
\gamma
\end{array}\right)=\left(\begin{array}{c}
-f_{1} k_{1} \rho+k_{1} f_{2}(1-\rho) \\
s \Phi-\left(k_{i}+k_{t}\right) \beta \\
k_{t} \beta / h-k_{e} \gamma
\end{array}\right)+\left(\begin{array}{lll}
0 & 0 & 0 \\
0 & 0 & 0 \\
0 & 0 & D
\end{array}\right) \cdot \nabla^{2}\left(\begin{array}{l}
\rho \\
\beta \\
\gamma
\end{array}\right)
$$

with $f_{1}, f_{2}, s$, and $\Phi$ defined in Section 1.3. Using the parameters of Chapter 5 and increasing the degradation $k_{e}$, the only steady state solution $\left(\rho_{0}, \beta_{0}, \gamma_{0}\right)^{T}$ has a Hopf bifurcation at $k_{e}^{*} \approx 4.3278 \mathrm{~min}^{-1}$. Around this point the amplitude equation was calculated. The first step involves calculating the derivatives up to third order, therefore, naming the reaction vector $F$, the first derivatives are

$$
\begin{array}{llrl}
\frac{\partial F_{1}}{\partial \rho} & =-\frac{k_{1}\left(L_{1}+L_{2} c \gamma \kappa\right)}{c \gamma+1}-\frac{k_{1}(\gamma \kappa+1)}{\gamma+1}, & & \frac{\partial F_{1}}{\partial \beta}=0, \\
\frac{\partial F_{1}}{\partial \gamma}=\frac{-k_{1} \rho(\kappa-1)}{(\gamma+1)^{2}}+\frac{c k_{1}(\rho-1)\left(L_{1}-L_{2} \kappa\right)}{(c \gamma+1)^{2}}, & \frac{\partial F_{2}}{\partial \rho}=\frac{2 \gamma^{2} \rho s\left(\lambda_{2}-\lambda_{1}\right)}{(\gamma+1)^{2}\left(\lambda_{2}+Y^{2}\right)^{2}}, \\
\frac{\partial F_{2}}{\partial \beta}=-\left(k_{i}+k_{t}\right), & \frac{\partial F_{2}}{\partial \gamma}=\frac{2 s \rho^{2} \gamma\left(\lambda_{2}-\lambda_{1}\right)}{(1+\gamma)^{3}\left(\lambda_{2}+Y^{2}\right)^{2}}, \\
\frac{\partial F_{3}}{\partial \rho}=0, & \frac{\partial F_{3}}{\partial \beta}=k_{t} / h, \text { and } \\
\frac{\partial F_{3}}{\partial \gamma}=-k_{e} . &
\end{array}
$$


The non-zero second derivatives are

$$
\begin{aligned}
\frac{\partial^{2} F_{1}}{\partial \gamma \partial \rho} & =\frac{-k_{1}(\kappa-1)}{(\gamma+1)^{2}}+\frac{c k_{1}\left(L_{1}-L_{2} \kappa\right)}{(c \gamma+1)^{2}}, \\
\frac{\partial^{2} F_{1}}{\partial \gamma^{2}} & =\frac{2 k_{1} \rho(\kappa-1)}{(\gamma+1)^{3}}-\frac{2 c^{2} k_{1}\left(L_{1}-L_{2} \kappa\right)(\rho-1)}{(c \gamma+1)^{3}}, \\
\frac{\partial^{2} F_{2}}{\partial \rho^{2}} & =\frac{2 \gamma^{2} s\left(\lambda_{2}-\lambda_{1}\right)}{(\gamma+1)^{2}\left(\lambda_{2}+Y^{2}\right)^{2}}+\frac{8 \gamma^{4} \rho^{2} s\left(\lambda_{1}-\lambda_{2}\right)}{(\gamma+1)^{4}\left(\lambda_{2}+Y^{2}\right)^{3}}, \\
\frac{\partial^{2} F_{2}}{\partial \rho \partial \gamma} & =\frac{4 \gamma \rho s\left(\lambda_{2}-\lambda_{1}\right)}{(\gamma+1)^{3}\left(\lambda_{2}+Y^{2}\right)^{2}}-\frac{8 \gamma^{3} \rho^{3} s\left(\lambda_{2}-\lambda_{1}\right)}{(\gamma+1)^{5}\left(\lambda_{2}+Y^{2}\right)^{3}}, \text { and } \\
\frac{\partial^{2} F_{2}}{\partial \gamma^{2}} & =\frac{2 s \rho^{2}\left(\lambda_{2}-\lambda_{1}\right)}{(1+\gamma)^{3}\left(\lambda_{2}+Y^{2}\right)^{2}}-\frac{6 s \rho^{2} \gamma\left(\lambda_{2}-\lambda_{1}\right)}{(1+\gamma)^{4}\left(\lambda_{2}+Y^{2}\right)^{2}}-\frac{8 s \rho^{4} \gamma^{2}\left(\lambda_{2}-\lambda_{1}\right)}{(1+\gamma)^{6}\left(\lambda_{2}+Y^{2}\right)^{3}} .
\end{aligned}
$$

And the non-zero third derivatives are

$$
\begin{aligned}
\frac{\partial^{3} F_{1}}{\partial \rho \partial \gamma^{2}}= & \frac{2 k_{1}(\kappa-1)}{(\gamma+1)^{3}}-\frac{2 c^{2} k_{1}\left(L_{1}-L_{2} \kappa\right)}{(c \gamma+1)^{3}}, \\
\frac{\partial^{3} F_{1}}{\partial \gamma^{3}}= & -\frac{6 k_{1} \rho(\kappa-1)}{(\gamma+1)^{4}}+\frac{6 c^{3} k_{1}\left(L_{1}-L_{2} \kappa\right)(\rho-1)}{(c \gamma+1)^{4}}, \\
\frac{\partial^{3} F_{2}}{\partial \rho^{3}}= & \frac{24 \gamma^{4} \rho s\left(\lambda_{1}-\lambda_{2}\right)}{(\gamma+1)^{4}\left(\lambda_{2}+Y^{2}\right)^{3}}-\frac{48 \gamma^{6} \rho^{3} s\left(\lambda_{1}-\lambda_{2}\right)}{(\gamma+1)^{6}\left(\lambda_{2}+Y^{2}\right)^{4}}, \\
\frac{\partial^{3} F_{2}}{\partial \gamma \partial \rho^{2}}= & \frac{4 \gamma s\left(\lambda_{2}-\lambda_{1}\right)}{(\gamma+1)^{3}\left(\lambda_{2}+Y^{2}\right)^{2}}-\frac{40 \gamma^{3} \rho^{2} s\left(\lambda_{2}-\lambda_{1}\right)}{(\gamma+1)^{5}\left(\lambda_{2}+Y^{2}\right)^{3}}+\frac{48 \gamma^{5} \rho^{4} s\left(\lambda_{2}-\lambda_{1}\right)}{(\gamma+1)^{7}\left(\lambda_{2}+Y^{2}\right)^{4}}, \\
\frac{\partial F_{2}}{\partial \rho \partial \gamma^{2}}= & \frac{4 s \rho\left(\lambda_{2}-\lambda_{1}\right)}{(1+\gamma)^{3}\left(\lambda_{2}+Y^{2}\right)^{2}}-\frac{8 s \rho^{3} \gamma^{2}\left(\lambda_{2}-\lambda_{1}\right)}{(1+\gamma)^{5}\left(\lambda_{2}+Y^{2}\right)^{3}}-\frac{12 s \rho \gamma\left(\lambda_{2}-\lambda_{1}\right)}{(1+\gamma)^{4}\left(\lambda_{2}+Y^{2}\right)^{2}} \\
& +\frac{24 s \rho^{3} \gamma^{3}\left(\lambda_{2}-\lambda_{1}\right)}{(1+\gamma)^{6}\left(\lambda_{2}+Y^{2}\right)^{3}}-\frac{32 s \rho^{3} \gamma^{2}\left(\lambda_{2}-\lambda_{1}\right)}{(1+\gamma)^{6}\left(\lambda_{2}+Y^{2}\right)^{3}}+\frac{48 s \rho^{5} \gamma^{4}\left(\lambda_{2}-\lambda_{1}\right)}{(1+\gamma)^{8}\left(\lambda_{2}+Y^{2}\right)^{4}}, \text { and } \\
\frac{\partial^{3} F_{2}}{\partial \gamma^{3}}= & -\frac{12 s \rho^{2}\left(\lambda_{2}-\lambda_{1}\right)}{(1+\gamma)^{4}\left(\lambda_{2}+Y^{2}\right)^{2}}-\frac{24 s \rho^{4} \gamma\left(\lambda_{2}-\lambda_{1}\right)}{(1+\gamma)^{6}\left(\lambda_{2}+Y^{2}\right)^{3}}+\frac{24 s \rho^{2} \gamma\left(\lambda_{2}-\lambda_{1}\right)}{(1+\gamma)^{5}\left(\lambda_{2}+Y^{2}\right)^{2}} \\
& +\frac{72 s \rho^{4} \gamma^{2}\left(\lambda_{2}-\lambda_{1}\right)}{(1+\gamma)^{7}\left(\lambda_{2}+Y^{2}\right)^{3}}+\frac{48 s \rho^{6} \gamma^{3}\left(\lambda_{2}-\lambda_{1}\right)}{(1+\gamma)^{9}\left(\lambda_{2}+Y^{2}\right)^{4}} .
\end{aligned}
$$

We define the Jacobian $\mathbf{J}$ using the first derivatives

$$
\mathbf{J}=J_{i j}=\frac{\partial F_{i}}{\partial x_{j}}
$$

At the bifurcation point two of the eigenvalues of $\mathbf{J}$ have zero real part, but non-zero imaginary part, thus crossing the imaginary axis. The third eigenvalue is a real negative number. The imaginary part of the crossing eigenvalues gives the frequency of the fast oscillation, $\omega_{0} \approx 1.4066 \mathrm{~min}^{-1}$, equivalent to $T \approx 4.467 \mathrm{~min}$. The eigenvectors of these 
a)

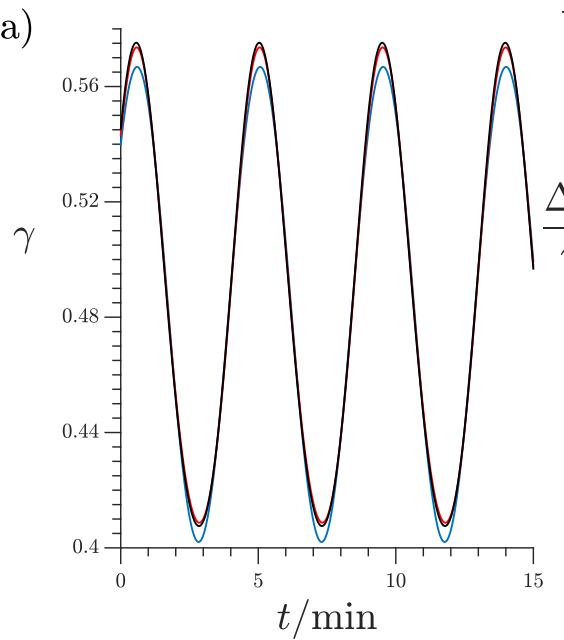

b) 0.02

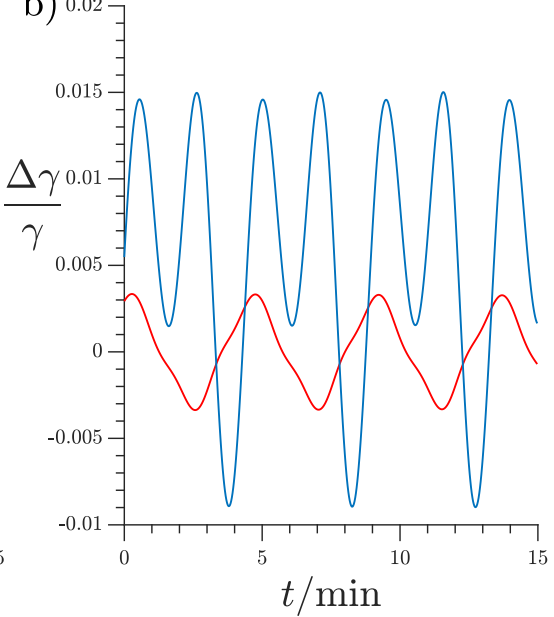

Figure A.1: Comparison of the the solution for the cAMP concentration given by Equation A.4 with the full non-linear simulation of the Martiel-Goldbeter model at $k_{e}=4.35 \mathrm{~min}^{-1}$, with a distance from the bifurcation of $\mu=0.0222$. a) Full simulation in black, approximation up to order $\sqrt{\mu}$ in blue, and up to order $\mu$ in red. b) Error in the approximation, same colors as in a).

eigenvalues at the Hopf bifurcation such that $\mathbf{J u}=i \omega_{0} \mathbf{u}$ and $\mathbf{u}^{\dagger} \mathbf{J}=i \omega_{0} \mathbf{u}^{\dagger}$ are

$$
\begin{aligned}
\mathbf{u} & \approx\left(\begin{array}{c}
-0.0016+0.0087 i \\
0.9992 \\
0.0376-0.0122 i
\end{array}\right) \text { and } \\
\mathbf{u}^{\dagger} & \approx(7.6202-56.7865 i, 0.3097-0.1182 i, 5.3967+0.7123 i),
\end{aligned}
$$

where these eigenvectors have been normalized such that $\mathbf{u}^{\dagger} \mathbf{u}=1$ and $\mathbf{u}^{\dagger} \overline{\mathbf{u}}=0$. Then we determine the vector coefficients $\mathbf{h}$ using the already calculated second derivatives and obtain

$$
\begin{gathered}
\mathbf{h}_{001}=-\mathbf{J}^{-1} \mathbf{F}_{k e} \approx\left(\begin{array}{c}
0.0295 \\
1.1694 \\
-0.0633
\end{array}\right), \quad \mathbf{h}_{110}=-\mathbf{J}^{-1} \mathbf{F}_{x x}(\mathbf{u}, \overline{\mathbf{u}}) \approx\left(\begin{array}{c}
0.0003 \\
0.0889 \\
0.0037
\end{array}\right), \\
\quad \text { and } \mathbf{h}_{200}=-\left(\mathbf{J}-2 i \omega_{0} \mathbf{I}\right)^{-1} \mathbf{F}_{x x}(\mathbf{u}, \mathbf{u}) / 2 \approx\left(\begin{array}{c}
-0.0001 \\
0.0551+0.0140 i \\
0.0019-0.0006 i
\end{array}\right),
\end{gathered}
$$

where

$$
\mathbf{F}_{x x}(\mathbf{u}, \mathbf{v})_{i}=\sum_{j, k} \frac{\partial^{2} F_{i}(x)}{\partial x_{j} \partial x_{k}} u_{j} v_{k} \quad \text { and } \quad \mathbf{F}_{k e}=\left(\begin{array}{c}
0 \\
0 \\
-\gamma_{0}
\end{array}\right)
$$



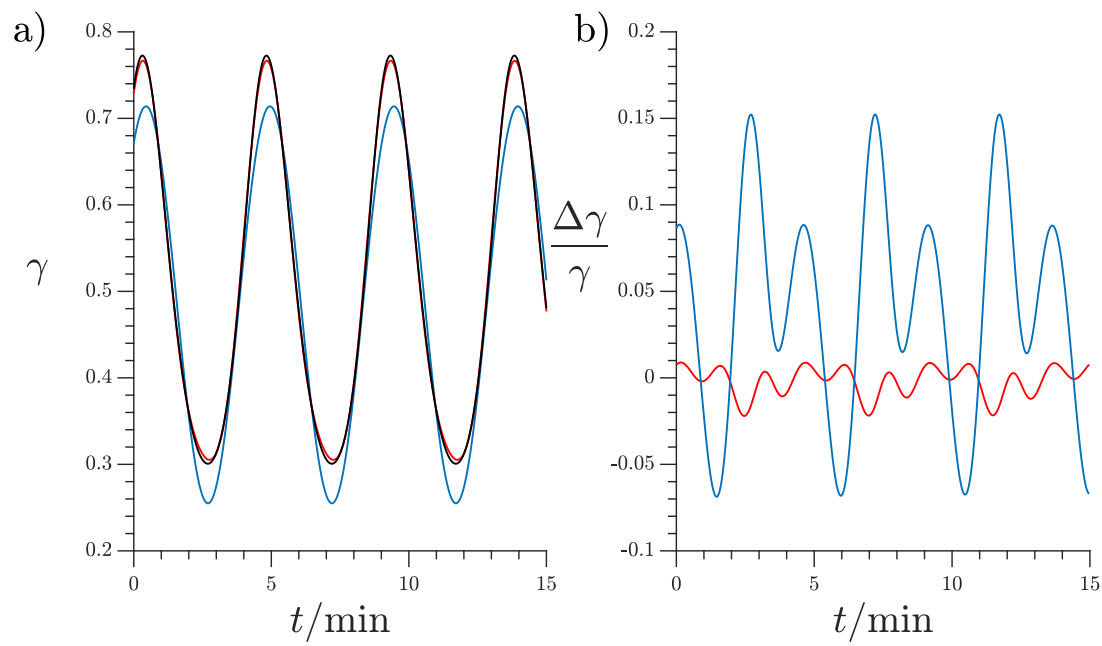

Figure A.2: Comparison of the the solution for the cAMP concentration given by Equation A.4 with the full non-linear simulation of the Martiel-Goldbeter model at $k_{e}=4.5 \mathrm{~min}^{-1}$, with a distance from the bifurcation of $\mu=0.1722$. a) Full simulation in black, approximation up to order $\sqrt{\mu}$ in blue, and up to order $\mu$ in red. b) Error in the approximation, same colors as in a).

Finally we calculate the resonant coefficients defined as

$$
\begin{aligned}
g & =-\mathbf{u}^{\dagger} \cdot\left(\mathbf{F}_{x x}\left(\mathbf{u}, \mathbf{h}_{110}\right)+\mathbf{F}_{x x}\left(\overline{\mathbf{u}}, \mathbf{h}_{200}\right)+\frac{1}{2} \mathbf{F}_{x x x}(\mathbf{u}, \mathbf{u}, \overline{\mathbf{u}})\right) \approx 0.0011+0.0012 i, \\
\sigma & =\mathbf{u}^{\dagger} \cdot\left(\mathbf{F}_{x k e} \cdot \mathbf{u}+\mathbf{F}_{x x}\left(\mathbf{u}, \mathbf{h}_{001}\right)\right) \approx 0.0515-0.0124 i, \\
d & =\mathbf{u}^{\dagger} \cdot \mathbf{D} \cdot \mathbf{u} \approx 0.0051-0.0009 i
\end{aligned}
$$

where $\mathbf{F}_{x k e}=\partial \mathbf{J} / \partial k_{e}$ and the third derivative of $\mathbf{F}$ is defined analogous to the second one. We then obtain the Complex Ginzburg-Landau Equation

$$
\dot{w}=\sigma\left(k_{e}-k_{e}^{*}\right) w-g|w|^{2} w+d \nabla^{2} w
$$

where $w$ is a complex variable describing the amplitude of the oscillation. Equation A.2 has the space independent solution

$$
w=\sqrt{\frac{\mu \sigma_{r}}{g_{r}}} \exp \left\{\mu\left(\sigma_{i}-\frac{\sigma_{r} g_{i}}{g_{r}}\right) t\right\}
$$

where $\mu=k_{e}-k_{e}^{*}, \sigma=\sigma_{r}+i \sigma_{i}$, and $g=g_{r}+i g_{i}$. The original variables are recovered up to order $\mu$ by the expression

$$
\left(\begin{array}{l}
\rho \\
\beta \\
\gamma
\end{array}\right)=\left(\begin{array}{c}
\rho_{0} \\
\beta_{0} \\
\gamma_{0}
\end{array}\right)+2 \operatorname{Re}\left\{w e^{i \omega_{0} t} \mathbf{u}\right\}+\mu \mathbf{h}_{001}+|w|^{2} \mathbf{h}_{110}+2 \operatorname{Re}\left\{\mathbf{h}_{200} w^{2} e^{2 i \omega_{0} t}\right\} .
$$



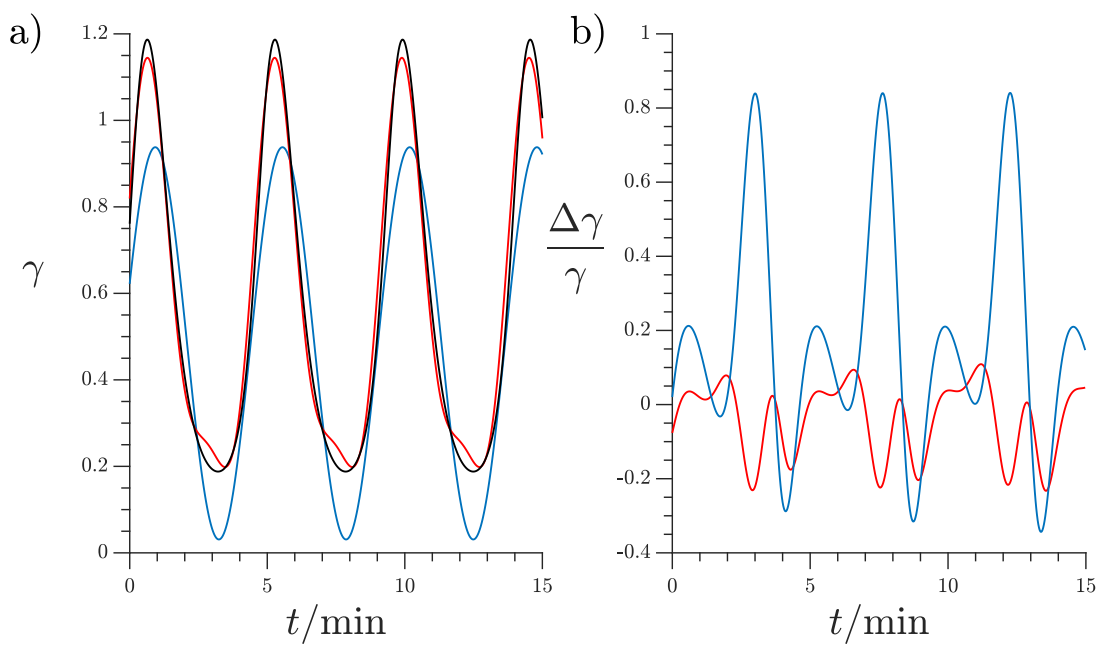

Figure A.3: Comparison of the the solution for the cAMP concentration given by Equation A.4 with the full non-linear simulation of the Martiel-Goldbeter model at $k_{e}=5.0 \mathrm{~min}^{-1}$, with a distance from the bifurcation of $\mu=0.6722$. a) Full simulation in black, approximation up to order $\sqrt{\mu}$ in blue, and up to order $\mu$ in red. b) Error in the approximation, same colors as in a).

Comparisons of Equation A.4 with the full nonlinear simulation are presented in Figure A.1, Figure A.2, and Figure A.3, where $\Delta \gamma=\left|\gamma-\gamma_{a}\right|$ with $\gamma_{a}$ given by Equation A.4. For $k_{e}=4.35 \mathrm{~min}^{-1}$ the error associated was up to $1.5 \%$ for the $\sqrt{\mu}$ order approximation and under $0.34 \%$ for the approximation $\mathcal{O}(\mu)$, due to the proximity to the bifurcation point the numerical simulation of the system took a long time to converge to the limit cycle, therefore Equation A.4 provides a good approximation than can save up a large amount of calculation time.

For $k_{e}=4.5 \mathrm{~min}^{-1}$ the corresponding errors were of $15.2 \%$ and $2.2 \%$. For $k_{e}=5.0$ $\min ^{-1}$ the errors were of the $83.5 \%$ and $23.27 \%$. At this last value of $k_{e}$ the numerical simulations converge pretty quickly to the limit cycle solution and considering the large errors associated with the Ginzburg-Landau approximation it does not provide a great advantage over computing the non-linear solution. At intermediate values of $k_{e}$ such as $k_{e}=4.5 \mathrm{~min}^{-1}$ the situation lies in between these two extreme cases and depending on the particular needs of the calculation both approaches can be used, with a the trade-off between calculation speed and accuracy. 



\section{List of Figures}

1.1 Processed dark-field microscopy images of a colony of cells during their development process. a) Initial circular pulses after $2.7 \mathrm{hrs}$ of starvation. b) Spiral patterns after 5 hrs of starvation. c) Initial aggregation patterns, 10 hrs of starvation. d) Clear aggregation streams, 13.9 hrs of starvation. (Courtesy of Torsten Eckstein at Max Planck Institute for Dynamics and Self-Organization, unpublished). . . . . . . . . . . . 4

1.2 Life cycle of $D$. discoideum showing the development that occurs under starvation conditions: pattern formation, aggregation, mound, slug, and fruiting body. Reproduced with permission of the publisher from the work of Chisholm and Firtel $[11] . \ldots \ldots \ldots \ldots$

1.3 Experimental observations of the Belousov-Zhabotinsky reaction, reproduced with permission of the publisher from the work of Zhabotinsky and Zaikin [43]. a) Target patterns. b) Spiral waves. . . . . . . . . . . . . . . 9

1.4 Numerical simulations of the Brusselator, Equation 1.1, showing oscillatory behavior at different parameters. All kinetic constants are taken as equal, $A=1$, and diffusion is neglected. a) $B=2.05$, b) $B=3.5$. Concentration of $X$ in black, concentration of $Y$ in red. . . . . . . . . 10

1.5 Typical features of oscillatory systems in the Brusselator model. a) Phase waves due to gradients in reactants' concentration. Concentration of $B$ increases linearly from 2.8 at $x=0$ to 3.2 at $x=10$. b) Center emitting target waves due to a higher frequency limit cycle. $B=2.5$ in $4.5<x<5.5, B=3.5$ everywhere else. $D_{X}=0.2, D_{Y}=0.02$. All other parameters as in Figure 1.4. Colormap by Peter Kovesi [53]. . . . . . . . 11

1.6 Two types of spirals that can be observed in oscillatory systems. a) Archimedian spiral. b) Logarithmic spiral. . . . . . . . . . . . . . . . . 12 
1.7 Excitable behavior in the FitzHugh-Nagumo model, Equation 1.3. Red simulation shows the reaction to an infra-threshold perturbation, blue simulation to a supra-threshold perturbation. a) Phase portrait showing both simulations and the nullclines $v=u / \beta$ and $v=u(u-\alpha)(1-u)$ in black. An infra-threshold perturbation (red line) decays quickly to the steady state $u_{0}=0, v_{0}=0$, a supra-threshold perturbation produces a big reaction from the system (blue line). b) Concentration of $u$ over time. Parameters are $\alpha=0.2, \beta=1.5, \epsilon=0.008$, initial conditions are for the red curve $u=0.24, v=0$ and for the blue curve $u=0.26, v=0 \ldots \ldots$

1.8 a) Example of a typical dispersion relation $c=f(T)$ for a planar trigger wave in an excitable system. $c$ is wave velocity and $T$ is the wave period. b) Example of an anomalous dispersion relation in the Kessler-Levine model. Part b) reproduced with minor modifications from the work of Oikawa et al. with permission of the publisher [63] . . . . . . . . . . 14

1.9 Phase diagram showing the different regimes in the Martiel-Goldbeter model with advection (Equation 1.7). a) 2-Component model. b) 3Component model also showing the path taken by the cells in the developmental path scheme used in Chapter 3 (Equation 1.6). Cells start in the stable regime and end in the excitable, at the parameters marked by the black circle. Reproduced with minor modifications from [76] . . . . . 18

1.10 Typical structures appearing during D. discoideum signaling, simulated using the model described by Equation 1.5 and Equation 1.6. a) Multiple circular waves. b) Spiral waves, on the bottom left a single armed spiral can be observed and on the right side there are two connected singlearmed spirals.

1.11 Nullclines for the 2-Component Martiel-Goldbeter model. $\partial_{t} \gamma=0$ in black and $\partial_{t} \rho=0$ in red. a) One solution at $k_{e}=4.0 \mathrm{~min}^{-1}$. b) Three solutions at $k_{e}=12 \mathrm{~min}^{-1} \ldots \ldots \ldots \ldots$

1.12 States of the Kessler-Levine Model. Transitions after a fixed time in the respective state are marked in blue, while transitions due to external cAMP concentration are marked in red. For all states the term sources in Equation 1.9 is 0, except for the excited State 1. . . . . . . . . . . . . 24

1.13 Limiting function $\psi$ used to discretize the advection operator. Used function $\psi=\max (0, \min (\theta, 1 / 3+\theta / 6,1))$ in bold red line. Dashed lines indicating $\psi=0, \psi=\theta, \psi=1 / 3+\theta / 6$, and $\psi=1$ are drawn as reference. The values of $\theta$ at which the discretization function reduces to known linear discretizations are marked with color in the background. . . . . . . 31 
3.1 Supplementary Figure 1 included with Influence of fast advective flows on pattern formation of Dictyostelium discoideum electronically available. Original caption "Flow profile in the channel. Laminar flow profile inside the microfluidic channel in arbitrary units. a) Cut along the channel center $(\mathrm{y}=0)$. b) Cut along half channel height $(\mathrm{z}=0) "[76] . \ldots 68$

A.1 Comparison of the the solution for the cAMP concentration given by Equation A.4 with the full non-linear simulation of the Martiel-Goldbeter model at $k_{e}=4.35 \mathrm{~min}^{-1}$, with a distance from the bifurcation of $\mu=$ 0.0222. a) Full simulation in black, approximation up to order $\sqrt{\mu}$ in blue, and up to order $\mu$ in red. b) Error in the approximation, same colors as in a)

A.2 Comparison of the the solution for the cAMP concentration given by Equation A.4 with the full non-linear simulation of the Martiel-Goldbeter model at $k_{e}=4.5 \mathrm{~min}^{-1}$, with a distance from the bifurcation of $\mu=$ 0.1722. a) Full simulation in black, approximation up to order $\sqrt{\mu}$ in blue, and up to order $\mu$ in red. b) Error in the approximation, same colors as in a). . . . . . . . . . . . . . . . . . . . . . . 112

A.3 Comparison of the the solution for the cAMP concentration given by Equation A.4 with the full non-linear simulation of the Martiel-Goldbeter model at $k_{e}=5.0 \mathrm{~min}^{-1}$, with a distance from the bifurcation of $\mu=$ 0.6722. a) Full simulation in black, approximation up to order $\sqrt{\mu}$ in blue, and up to order $\mu$ in red. b) Error in the approximation, same colors as in a). . . . . . . . . . . . . . . . . . . . . . 113 



\section{Bibliography}

[1] R. H. Kessin. Dictyostelium: evolution, cell biology, and the development of multicellularity. Vol. 38. Cambridge University Press, 2001.

[2] J. T. Bonner. Cellular Slime Molds. Vol. 2127. Princeton University Press, 1959.

[3] W. Loomis. Dictyostelium discoideum: a developmental system. Elsevier, 1975.

[4] J. M. Mato, A. Losada, V. Nanjundiah and T. M. Konijn. "Signal input for a chemotactic response in the cellular slime mold Dictyostelium discoideum". Proceedings of the National Academy of Sciences 72.12 (1975), pp. 4991-4993. DOI: 10.1073/pnas.72.12.4991.

[5] M. Skoge, H. Yue, M. Erickstad, A. Bae, H. Levine, A. Groisman, W. F. Loomis and W.-J. Rappel. "Cellular memory in eukaryotic chemotaxis". Proceedings of the National Academy of Sciences 111.40 (2014), pp. 14448-14453. DOI: 10.1073/pnas.1412197111.

[6] A. Nakajima, S. Ishihara, D. Imoto and S. Sawai. "Rectified directional sensing in long-range cell migration". Nature communications 5 (2014), p. 5367. DOI: 10.1038/ncomms6367.

[7] J. Gross, M. Peacey and D. Trevan. "Signal emission and signal propagation during early aggregation in Dictyostelium discoideum". Journal of cell science 22.3 (1976), pp. 645-656.

[8] M. Katoh, G. Chen, E. Roberge, G. Shaulsky and A. Kuspa. "Developmental commitment in Dictyostelium discoideum". Eukaryotic cell 6.11 (2007), pp. 2038-2045. DOI: 10 . 1128/EC . 00223-07.

[9] K. B. Raper. "Pseudoplasmodium formation and organization in Dictyostelium discoideum". Journal of the Elisha Mitchell Scientific Society 56.2 (1940), pp. 241-282.

[10] J. T. Bonner, W. W. Clarke Jr., C. L. Neely Jr. and M. K. Slifkin. "The orientation to light and the extremely sensitive orientation to temperature gradients in the slime mold Dictyostelium discoideum". Journal of cellular and comparative physiology 36.2 (1950), pp. 149-158. DOI: $10.1002 /$ jcp. 1030360203.

[11] R. L. Chisholm and R. A. Firtel. "Insights into morphogenesis from a simple developmental system". Nature reviews. Molecular cell biology 5.7 (2004), p. 531. DOI: 10.1038/nrm1427.

[12] O. Brefeld. "Dictyostelium mucoroides. Ein neuer Organismus aus der Verwandshaft der Myxomyceten". Abhandl. Senckenbergish Naturf. Ges. 7 (1869), pp. 85-107.

[13] K. B. Raper. "Dictyostelium discoideum, a new species of slime mold from decaying forest leaves". J. Agricul. Res. 50 (1935), pp. 135-147.

[14] K. B. Raper and C. Thom. "Interspecific mixtures in the Dictyosteliaceae". American Journal of Botany 28.1 (1941), pp. 69-78. DOI: 10.1002/j.1537-2197.1941.tb07944.x. 
[15] J. T. Bonner and L. Savage. "Evidence for the formation of cell aggregates by chemotaxis in the development of the slime mold Dictyostelium discoideum". Journal of Experimental Zoology 106.1 (1947), pp. 1-26. DOI: 10.1002/jez.1401060102.

[16] T. M. Konijn. "Chemotaxis in the cellular slime molds. II. The effect of cell density". The Biological Bulletin 134.2 (1968), pp. 298-304. DOI: 10.2307/1539605.

[17] B. Shaffer. "The acrasina". In: Advances in morphogenesis. Vol. 2. Elsevier, 1962, pp. 109-182. DOI: $10.1016 / \mathrm{B} 978-1-4831-9949-8.50007-9$.

[18] P. Klein, R. Vaughan, J. Borleis and P. Devreotes. "The surface cyclic AMP receptor in Dictyostelium. Levels of ligand-induced phosphorylation, solubilization, identification of primary transcript, and developmental regulation of expression." Journal of Biological Chemistry 262.1 (1987), pp. 358-364.

[19] T. J. Sun and P. N. Devreotes. "Gene targeting of the aggregation stage cAMP receptor cAR1 in Dictyostelium." Genes E3 Development 5.4 (1991), pp. 572-582. DOI: 10.1101/gad.5.4.572.

[20] R. L. Johnson, C. L. Saxe, R. Gollop, A. R. Kimmel and P. N. Devreotes. "Identification and targeted gene disruption of cAR3, a cAMP receptor subtype expressed during multicellular stages of Dictyostelium development." Genes \& development 7.2 (1993), pp. 273-282. DOI: 10. 1101/gad.7.2.273.

[21] J. M. Louis, G. T. Ginsburg and A. R. Kimmel. "The cAMP receptor cAR4 regulates axial patterning and cellular differentiation during late development of Dictyostelium." Genes 83 Development 8.17 (1994), pp. 2086-2096. DOI: 10.1101/gad.8.17.2086.

[22] C. L. Saxe, G. T. Ginsburg, J. M. Louis, R. Johnson, P. N. Devreotes and A. R. Kimmel. "cAR2, a prestalk cAMP receptor required for normal tip formation and late development of Dictyostelium discoideum." Genes \&3 Development 7.2 (1993), pp. 262-272. DoI: 10.1101/gad. 7.2.262.

[23] C. Klein and M. H. Juliani. "cAMP-induced changes in cAMP-binding sites on D. discoideum amebae". Cell 10.2 (1977), pp. 329-335. DOI: 10.1016/0092-8674(77)90227-6.

[24] D. Hereld, R. Vaughan, J. Y. Kim, J. Borleis and P. Devreotes. "Localization of ligand-induced phosphorylation sites to serine clusters in the C-terminal domain of the Dictyostelium cAMP receptor, cAR1." Journal of Biological Chemistry 269.9 (1994), pp. 7036-7044.

[25] P. Devreotes and J. Sherring. "Kinetics and concentration dependence of reversible cAMPinduced modification of the surface cAMP receptor in Dictyostelium." Journal of Biological Chemistry 260.10 (1985), pp. 6378-6384.

[26] M. C. Dinauer, S. A. MacKay and P. N. Devreotes. "Cyclic 3', 5'-AMP relay in Dictyostelium discoideum III. The relationship of cAMP synthesis and secretion during the cAMP signaling response." The Journal of Cell Biology 86.2 (1980), pp. 537-544. DOI: 10.1083/jcb.86.2.537.

[27] P. N. Devreotes, S. Bhattacharya, M. Edwards, P. A. Iglesias, T. Lampert and Y. Miao. "Excitable signal transduction networks in directed cell migration". Annual review of cell and developmental biology 33 (2017), pp. 103-125. DOI: 10.1146/annurev-cellbio-100616-060739.

[28] G. Gerisch and D. Malchow. "Cyclic AMP receptors and the control of cell aggregation in Dictyostelium." Advances in cyclic nucleotide research 7 (1976), p. 49. 
[29] R. Insall, A. Kuspa, P. J. Lilly, G. Shaulsky, L. R. Levin, W. F. Loomis and P. Devreotes. "CRAC, a cytosolic protein containing a pleckstrin homology domain, is required for receptor and G protein-mediated activation of adenylyl cyclase in Dictyostelium." The Journal of Cell Biology 126.6 (1994), pp. 1537-1545. DOI: 10.1083/jcb.126.6.1537.

[30] S. van Es, K. J. Virdy, G. S. Pitt, M. Meima, T. W. Sands, P. N. Devreotes, D. A. Cotter and P. Schaap. "Adenylyl cyclase G, an osmosensor controlling germination of Dictyostelium spores". Journal of Biological Chemistry 271.39 (1996), pp. 23623-23625. DOI: 10.1074/jbc.271.39. 23623.

[31] M. E. Meima and P. Schaap. "Fingerprinting of adenylyl cyclase activities during Dictyostelium development indicates a dominant role for adenylyl cyclase B in terminal differentiation". Developmental biology 212.1 (1999), pp. 182-190. DOI: 10.1006/dbio.1999.9352.

[32] S. Bader, A. Kortholt and P. J. Van Haastert. "Seven Dictyostelium discoideum phosphodiesterases degrade three pools of cAMP and cGMP". Biochemical Journal 402.1 (2007), pp. 153161. DOI: $10.1042 / B J 20061153$.

[33] T. Eckstein, E. Vidal-Henriquez, A. Bae and A. Gholami. "Spatial heterogeneities shape collective behavior of signaling amoeboid cells". arXiv preprint arXiv:1804.06686 (2018).

[34] M. Brenner and S. D. Thoms. "Caffeine blocks activation of cyclic AMP synthesis in Dictyostelium discoideum". Developmental biology 101.1 (1984), pp. 136-146. DoI: 10.1016/0012$1606(84) 90124-6$.

[35] A. Theibert and P. N. Devreotes. "Cyclic 3', 5'-AMP relay in Dictyostelium discoideum: adaptation is independent of activation of adenylate cyclase." The Journal of cell biology 97.1 (1983), pp. 173-177. DOI: $10.1083 /$ jcb.97.1.173.

[36] F. Siegert and C. Weijer. "Digital image processing of optical density wave propagation in Dictyostelium discoideum and analysis of the effects of caffeine and ammonia". Journal of Cell Science 93.2 (1989), pp. 325-335.

[37] E. Alvarez-Curto, K. E. Weening and P. Schaap. "Pharmacological profiling of the Dictyostelium adenylate cyclases ACA, ACB and ACG". Biochemical Journal 401.1 (2007), pp. 309-316. DOI: 10.1042/BJ20060880.

[38] B. Belousov. "Periodicheski deistvuyushchaya reaktsia i ee mekhanism (Periodically acting reaction and its mechanism)". Sbornik Referatov po Radiotsionnoi Meditsine (1958), p. 145.

[39] B. Belousov. "A periodic reaction and its mechanism". In: Oscillations and traveling waves in chemical systems. Ed. by R. J. Field and M. Burger. Wiley, 1985.

[40] I. R. Epstein and J. A. Pojman. An introduction to nonlinear chemical dynamics: oscillations, waves, patterns, and chaos. Oxford University Press, 1998.

[41] R. Lefever. "The rehabilitation of irreversible processes and dissipative structures' 50th anniversary". Phil. Trans. R. Soc. A 376.2124 (2018), p. 20170365. DOI: 10.1098/rsta.2017.0365.

[42] A. Zaikin and A. Zhabotinsky. "Concentration wave propagation in two-dimensional liquidphase self-oscillating system”. Nature 225 (1970), pp. 535-537. DOI: 10.1038/225535b0.

[43] A. Zhabotinsky and A. Zaikin. "Autowave processes in a distributed chemical system". Journal of theoretical biology 40.1 (1973), pp. 45-61. DOI: 10.1016/0022-5193(73)90164-1.

[44] I. Prigogine and R. Lefever. "Symmetry breaking instabilities in dissipative systems. II". The Journal of Chemical Physics 48.4 (1968), pp. 1695-1700. DOI: 10.1063/1.1668896. 
[45] R. J. Field, E. Koros and R. M. Noyes. "Oscillations in chemical systems. II. Thorough analysis of temporal oscillation in the bromate-cerium-malonic acid system". Journal of the American Chemical Society 94.25 (1972), pp. 8649-8664. DOI: 10.1021/ja00780a001.

[46] V. Volterra. Variazioni e fluttuazioni del numero d'individui in specie animali conviventi. C. Ferrari, 1927.

[47] N. Kopell and L. Howard. "Horizontal bands in the Belousov reaction". Science 180.4091 (1973), pp. 1171-1173. DOI: 10.1126/science.180.4091.1171.

[48] N. Kopell and L. Howard. "Plane wave solutions to reaction-diffusion equations". Studies in Applied Mathematics 52.4 (1973), pp. 291-328. DOI: 10.1002/sapm1973524291.

[49] A. E. Bugrim, M. Dolnik, A. M. Zhabotinsky and I. R. Epstein. "Heterogeneous sources of target patterns in reaction- diffusion systems". The Journal of Physical Chemistry 100.49 (1996), pp. 19017-19022. DOI: 10.1021/jp961603t.

[50] M. Stich and A. S. Mikhailov. "Complex pacemakers and wave sinks in heterogeneous oscillatory chemical systems". Zeitschrift für Physikalische Chemie 216.4 (2002), p. 521. DOI: 10.1524/ zpch.2002.216.4.521.

[51] Y. Kuramoto. Chemical oscillations, waves, and turbulence. Vol. 19. Springer Science \& Business Media, 2012.

[52] J. J. Tyson and P. C. Fife. "Target patterns in a realistic model of the Belousov-Zhabotinskii reaction". The Journal of Chemical Physics 73.5 (1980), pp. 2224-2237. DoI: 10 . 1063/1. 440418.

[53] P. Kovesi. "Good colour maps: How to design them". arXiv preprint arXiv:1509.03700 (2015).

[54] D. S. Cohen, J. C. Neu and R. R. Rosales. "Rotating spiral wave solutions of reaction-diffusion equations". SIAM journal on applied mathematics 35.3 (1978), pp. 536-547. DOI: 10.1137 / 0135045.

[55] J. Greenberg. "Spiral waves for $\lambda$ - $\omega$ systems". SIAM journal on applied mathematics 39.2 (1980), pp. 301-309. DOI: $10.1137 / 0139026$.

[56] D. Dormann, B. Vasiev and C. J. Weijer. "Propagating waves control Dictyostelium discoideum morphogenesis". Biophysical chemistry 72.1-2 (1998), pp. 21-35. DOI: 10.1016/S0301-4622 (98) 00120-3.

[57] L. D. Landau. "On the problem of turbulence". In: Dokl. Akad. Nauk SSSR. Vol. 44. 8. 1944, pp. 339-349.

[58] M. Ipsen, L. Kramer and P. G. Sørensen. "Amplitude equations for description of chemical reaction-diffusion systems". Physics Reports 337.1-2 (2000), pp. 193-235. DOI: 10.1016/S03701573(00)00062-4.

[59] I. S. Aranson and L. Kramer. "The world of the complex Ginzburg-Landau equation". Reviews of Modern Physics 74.1 (2002), p. 99. DOI: 10.1103/RevModPhys.74.99.

[60] A. T. Winfree. "Spiral waves of chemical activity". Science 175.4022 (1972), pp. 634-636. DoI: $10.1126 /$ science. 175.4022 .634 .

[61] A. L. Hodgkin and A. F. Huxley. "A quantitative description of membrane current and its application to conduction and excitation in nerve". The Journal of physiology 117.4 (1952), pp. 500-544. DOI: 10.1113/jphysiol.1952.sp004764. 
[62] M. A. Allessie, F. I. Bonke and F. J. Schopman. "Circus movement in rabbit atrial muscle as a mechanism of tachycardia". Circulation research 33.1 (1973), pp. 54-62. DOI: 10.1161/01. RES.33.1.54.

[63] N. Oikawa, E. Bodenschatz and V. Zykov. "Unusual spiral wave dynamics in the Kessler-Levine model of an excitable medium". Chaos: An Interdisciplinary Journal of Nonlinear Science 25.5 (2015), p. 053115. DOI: 10.1063/1.4921879.

[64] R. R. Aliev and V. N. Biktashev. "Dynamics of the oscillation phase distribution in the BZ reaction". The Journal of Physical Chemistry 98.38 (1994), pp. 9676-9681. DOI: $10.1021 /$ j100089a049.

[65] J. J. Tyson and J. P. Keener. "Singular perturbation theory of traveling waves in excitable media (a review)". Physica D: Nonlinear Phenomena 32.3 (1988), pp. 327-361. DOI: 10.1016/01672789 (88) $90062-0$.

[66] P. C. Fife. "Understanding the patterns in the BZ reagent". Journal of Statistical Physics 39.5-6 (1985), pp. 687-703. DOI: 10.1007/BF01008360.

[67] A. Karma. "Scaling regime of spiral wave propagation in single-diffusive media". Physical review letters 68.3 (1992), p. 397. DOI: 10.1103/PhysRevLett.68.397.

[68] V. Zykov. "Kinematics of rigidly rotating spiral waves". Physica D: Nonlinear Phenomena 238.11-12 (2009), pp. 931-940. DOI: 10.1016/j.physd.2008.06.009.

[69] R. FitzHugh. "Impulses and physiological states in theoretical models of nerve membrane". Biophysical journal 1.6 (1961), pp. 445-466. DOI: 10.1016/S0006-3495(61)86902-6.

[70] J. Nagumo, S. Arimoto and S. Yoshizawa. "An active pulse transmission line simulating nerve axon". Proceedings of the IRE 50.10 (1962), pp. 2061-2070. DOI: 10.1109/JRPROC.1962. 288235.

[71] D. Barkley. "A model for fast computer simulation of waves in excitable media". Physica D: Nonlinear Phenomena 49.1-2 (1991), pp. 61-70. DOI: 10.1016/0167-2789(91)90194-E.

[72] V. S. Zykov. Simulation of wave processes in excitable media. Manchester Univ Press, Manchester, UK, 1987.

[73] J. P. Keener. "A geometrical theory for spiral waves in excitable media". SIAM Journal on Applied Mathematics 46.6 (1986), pp. 1039-1056. DOI: 10.1137/0146062.

[74] P. C. Fife. Mathematical aspects of reacting and diffusing systems. Vol. 28. Springer Science \& Business Media, 2013.

[75] J.-L. Martiel and A. Goldbeter. "A model based on receptor desensitization for cyclic AMP signaling in Dictyostelium cells". Biophysical Journal 52.5 (1987), p. 807. DOI: 10.1016/S00063495 (87) $83275-7$.

[76] T. Eckstein, E. Vidal-Henriquez, A. Bae, V. Zykov, E. Bodenschatz and A. Gholami. "Influence of fast advective flows on pattern formation of Dictyostelium discoideum". PloS one 13.3 (2018), e0194859. DOI: 10.1371/journal pone.0194859.

[77] J. J. Tyson, K. A. Alexander, V. Manoranjan and J. Murray. "Spiral waves of cyclic AMP in a model of slime mold aggregation". Physica D: Nonlinear Phenomena 34.1-2 (1989), pp. 193-207. DOI: $10.1016 / 0167-2789$ (89)90234-0. 
[78] J. Lauzeral, J. Halloy and A. Goldbeter. "Desynchronization of cells on the developmental path triggers the formation of spiral waves of cAMP during Dictyostelium aggregation". Proceedings of the National Academy of Sciences 94.17 (1997), pp. 9153-9158. DOI: 10.1073/pnas.94.17. 9153.

[79] C. Klein. "Changes in adenylate cyclase during differentiation of Dictyostelium discoideum". FEMS Microbiology Letters 1.1 (1977), pp. 17-19. DoI: 10.1016/0378-1097 (77)90102-1.

[80] D. Malchow, B. Nägele, H. Schwarz and G. Gerisch. "Membrane-bound cyclic AMP phosphodiesterase in chemotactically responding cells of Dictyostelium discoideum". European Journal of Biochemistry 28.1 (1972), pp. 136-142. DOI: 10.1111/j.1432-1033.1972.tb01894.x.

[81] R. Yeh, F. Chan and M. Coukell. "Independent regulation of the extracellular cyclic AMP phosphodiesterase-inhibitor system and membrane differentiation by exogenous cyclic AMP in Dictyostelium discoideum". Developmental biology 66.2 (1978), pp. 361-374. DOI: 10.1016/ 0012-1606 (78) 90245-2.

[82] D. Geberth and M.-T. Hütt. "Predicting the distribution of spiral waves from cell properties in a developmental-path model of Dictyostelium pattern formation". PLoS computational biology 5.7 (2009), e1000422. DOI: 10.1371/journal.pcbi. 1000422.

[83] M. Grace and M.-T. Hütt. "Regulation of spatiotemporal patterns by biological variability: general principles and applications to Dictyostelium discoideum". PLoS computational biology 11.11 (2015), e1004367. DOI: 10.1371/journal.pcbi.1004367.

[84] E. Palsson and E. C. Cox. "Origin and evolution of circular waves and spirals in Dictyostelium discoideum territories". Proceedings of the National Academy of Sciences 93.3 (1996), pp. 11511155. DOI: $10.1073 /$ pnas.93.3.1151.

[85] J. Lindner, H. Ševčíková and M. Marek. "Influence of an external electric field on cAMP wave patterns in aggregating Dictyostelium discoideum". Physical Review E 63.4 (2001), p. 041904. DOI: $10.1103 /$ PhysRevE.63.041904.

[86] A. Gholami, O. Steinbock, V. Zykov and E. Bodenschatz. "Flow-driven instabilities during pattern formation of Dictyostelium discoideum". New Journal of Physics 17.6 (2015), p. 063007. DOI: $10.1088 / 1367-2630 / 17 / 6 / 063007$.

[87] A. Gholami, V. Zykov, O. Steinbock and E. Bodenschatz. "Flow-driven two-dimensional waves in colonies of Dictyostelium discoideum". New Journal of Physics 17.9 (2015), p. 093040. DoI: 10.1088/1367-2630/17/9/093040.

[88] H. Ševčíková and M. Marek. "Chemical waves in electric field". Physica D: Nonlinear Phenomena 9.1-2 (1983), pp. 140-156. DOI: 10.1016/0167-2789 (83) 90296-8.

[89] H. Ševčiková and M. Marek. "Chemical waves in electric field-modelling". Physica D: Nonlinear Phenomena 21.1 (1986), pp. 61-77. DOI: 10.1016/0167-2789 (86)90078-3.

[90] O. Steinbock, J. Schütze and S. Müller. "Electric-field-induced drift and deformation of spiral waves in an excitable medium". Physical review letters 68.2 (1992), p. 248. DOI: 10.1103/ PhysRevLett.68.248.

[91] K. Agladze and P. De Kepper. "Influence of electric field on rotating spiral waves in the BelousovZhabotinsky reaction". The Journal of Physical Chemistry 96.13 (1992), pp. 5239-5242. DOI: $10.1021 / \mathrm{j} 100192 \mathrm{a} 015$. 
[92] K. Agladze, M. Braune, H. Engel, H. Linde and V. Krinsky. "Autowave propagation in a Belousov-Zhabotinsky medium with immobilized catalyst and stationary flow of reagents". Zeitschrift für Physikalische Chemie 173.1 (1991), pp. 79-85. DOI: 10.1524/zpch.1991.173.Part_ 1.079 .

[93] A. B. Rovinsky and M. Menzinger. "Self-organization induced by the differential flow of activator and inhibitor". Physical Review Letters 70.6 (1993), p. 778. DOI: 10.1103/PhysRevLett.70.778.

[94] R. Toth, A. Papp, V. Gaspar, J. Merkin, S. Scott and A. Taylor. "Flow-driven instabilities in the Belousov-Zhabotinsky reaction: Modelling and experiments". Physical Chemistry Chemical Physics 3.6 (2001), pp. 957-964. DOI: 10.1039/B009379N.

[95] E. Vidal-Henriquez, V. Zykov, E. Bodenschatz and A. Gholami. "Convective instability and boundary driven oscillations in a reaction-diffusion-advection model". Chaos: An Interdisciplinary Journal of Nonlinear Science 27.10 (2017), p. 103110. DOI: 10.1063/1.4986153.

[96] D. A. Kessler and H. Levine. "Pattern formation in Dictyostelium via the dynamics of cooperative biological entities". Physical Review E 48.6 (1993), p. 4801. DOI: 10.1103/PhysRevE. 48. 4801.

[97] H. Levine, I. Aranson, L. Tsimring and T. V. Truong. "Positive genetic feedback governs cAMP spiral wave formation in Dictyostelium". Proceedings of the National Academy of Sciences 93.13 (1996), pp. 6382-6386. DOI: 10.1073/pnas.93.13.6382.

[98] H. Levine. "Pattern formation in the microbial world: Dictyostelium discoideum". In: Epilepsy as a dynamic disease. Ed. by J. Milton and P. Jung. Springer, 2003, pp. 189-211.

[99] D. Geberth and M.-T. Hütt. "Predicting spiral wave patterns from cell properties in a model of biological self-organization". Physical Review E 78.3 (2008), p. 031917. DOI: 10.1103/PhysRevE. 78.031917.

[100] S. Sawai, P. A. Thomason and E. C. Cox. "An autoregulatory circuit for long-range selforganization in Dictyostelium cell populations". Nature 433.7023 (2005), pp. 323-326. DOI: 10.1038/nature03228.

[101] T. Höfer, J. A. Sherratt and P. K. Maini. "Dictyostelium discoideum: Cellular self-organization in an excitable biological medium". Proceedings of the Royal Society of London B: Biological Sciences 259.1356 (1995), pp. 249-257. DOI: 10.1098/rspb.1995.0037.

[102] T. Höfer, J. A. Sherratt and P. K. Maini. "Cellular pattern formation during Dictyostelium aggregation". Physica D: Nonlinear Phenomena 85.3 (1995), pp. 425-444. DOI: 10.1016/01672789 (95) $00075-\mathrm{F}$.

[103] E. F. Keller and L. A. Segel. "Initiation of slime mold aggregation viewed as an instability". Journal of theoretical biology 26.3 (1970), pp. 399-415. DOI: 10.1016/0022-5193(70)90092-5.

[104] D. R. Soll, D. Wessels and A. Sylwester. "The motile behavior of amoebae in the aggregation wave in Dictyostelium discoideum". In: Experimental and Theoretical Advances in Biological Pattern Formation. Ed. by H. G. Othmer, P. K. Maini and J. D. Murray. Boston, MA: Springer US, 1993, pp. 325-338.

[105] B. Vasiev, P Hogeweg and A. Panfilov. "Simulation of Dictyostelium discoideum aggregation via reaction-diffusion model". Physical Review Letters 73.23 (1994), p. 3173. DOI: $10.1103 /$ PhysRevLett.73.3173. 
[106] C. Van Oss, A. V. Panfilov, P. Hogeweg, F. Siegert and C. J. Weijer. "Spatial pattern formation during aggregation of the slime mould Dictyostelium discoideum". Journal of theoretical biology 181.3 (1996), pp. 203-213. DOI: 10.1006/jtbi.1996.0126.

[107] E. Vidal-Henriquez and A. Gholami. "Spontaneous center formation in Dictyostelium discoideum". Scientific reports 9.1 (2019), p. 3935. DOI: 10.1038/s41598-019-40373-4.

[108] R. Merson. "An operational method for the study of integration processes". In: Proc. Symp. Data Processing. 1957, pp. 1-25.

[109] W. Hundsdorfer and J. G. Verwer. Numerical solution of time-dependent advection-diffusionreaction equations. Vol. 33. Springer Science \& Business Media, 2013.

[110] B. Koren. "A robust upwind discretization method for advection, diffusion and source terms". In: Numerical methods for advection-diffusion problems. Ed. by C. B. Vreugdenhil. Vieweg, 1993.

[111] R. J. Deissler. "Noise-sustained structure, intermittency, and the Ginzburg-Landau equation". Journal of statistical physics 40.3-4 (1985), pp. 371-395. DOI: 10.1007/BF01017180.

[112] C. T. Hamik and O. Steinbock. "Shock structures and bunching fronts in excitable reactiondiffusion systems". Physical Review E 65.4 (2002), p. 046224. DOI: 10.1103/PhysRevE. 65. 046224.

[113] N. Manz and O. Steinbock. "Dynamics of excitation pulses with attractive interaction: Kinematic analysis and chemical wave experiments". Physical Review E 70.6 (2004), p. 066213. DoI: 10. 1103/PhysRevE.70.066213.

[114] B. F. Edwards. "Poiseuille advection of chemical reaction fronts". Physical Review Letters 89.10 (2002), p. 104501. DOI: 10.1103/PhysRevLett.89.104501.

[115] M. Leconte, J. Martin, N. Rakotomalala and D. Salin. "Pattern of reaction diffusion fronts in laminar flows". Physical Review Letters 90.12 (2003), p. 128302. DOI: 10.1103/PhysRevLett. 90.128302 .

[116] E. A. Ermakova, E. E. Shnol, M. A. Panteleev, A. A. Butylin, V. Volpert and F. I. Ataullakhanov. "On propagation of excitation waves in moving media: The FitzHugh-Nagumo model". PloS One 4.2 (2009), e4454. DOI: $10.1371 /$ journal pone.0004454.

[117] M. P. Mosley. "Subsurface flow velocities through selected forest soils, South Island, New Zealand". Journal of hydrology 55.1-4 (1982), pp. 65-92. DOI: 10.1016/0022-1694(82) 90121-4.

[118] P. N. Devreotes, M. J. Potel and S. A. MacKay. "Quantitative analysis of cyclic AMP waves mediating aggregation in Dictyostelium discoideum". Developmental biology 96.2 (1983), pp. 405415. DOI: $10.1016 / 0012-1606$ (83) $90178-1$.

[119] P. Jaiswal, T. Soldati, S. Thewes and R. Baskar. "Regulation of aggregate size and pattern by adenosine and caffeine in cellular slime molds". BMC developmental biology 12.1 (2012), p. 5. DOI: $10.1186 / 1471-213 \mathrm{X}-12-5$.

[120] K. H. Prabhakara, A. Gholami, V. S. Zykov and E. Bodenschatz. "Effects of developmental variability on the dynamics and self-organization of cell populations". New Journal of Physics 19.11 (2017), p. 113024. DOI: 10.1088/1367-2630/aa9391.

[121] M. W. Toepke and D. J. Beebe. "PDMS absorption of small molecules and consequences in microfluidic applications". Lab on a Chip 6.12 (2006), pp. 1484-1486. DOI: 10.1039/B612140C. 
[122] O. Steinbock and S. Müller. "Chemical spiral rotation is controlled by light-induced artificial cores". Physica A: Statistical Mechanics and its Applications 188.1-3 (1992), pp. 61-67. DOI: 10.1016/0378-4371(92) 90253-M.

[123] A. P. Munuzuri, V. Pérez-Muñuzuri and V. Pérez-Villar. "Attraction and repulsion of spiral waves by localized inhomogeneities in excitable media". Physical Review E 58.3 (1998), R2689. DOI: 10.1103/PhysRevE.58.R2689.

[124] M. Sutthiopad, J. Luengviriya, P. Porjai, M. Phantu, J. Kanchanawarin, S. C. Müller and C. Luengviriya. "Propagation of spiral waves pinned to circular and rectangular obstacles". Physical Review E 91.5 (2015), p. 052912. DOI: 10.1103/PhysRevE.91.052912.

[125] Z. A. Jiménez, Z. Zhang and O. Steinbock. "Electric-field-controlled unpinning of scroll waves". Physical Review E 88.5 (2013), p. 052918. DOI: 10.1103/PhysRevE.88.052918.

[126] M. Sutthiopad, J. Luengviriya, P. Porjai, B. Tomapatanaget, S. C. Müller and C. Luengviriya. "Unpinning of spiral waves by electrical forcing in excitable chemical media". Physical Review E 89.5 (2014), p. 052902. DOI: 10.1103/PhysRevE.89.052902.

[127] P. W. Kriebel, V. A. Barr and C. A. Parent. "Adenylyl cyclase localization regulates streaming during chemotaxis". Cell 112.4 (2003), pp. 549-560. DOI: 10.1016/S0092-8674(03)00081-3.

[128] P. W. Kriebel, V. A. Barr, E. C. Rericha, G. Zhang and C. A. Parent. "Collective cell migration requires vesicular trafficking for chemoattractant delivery at the trailing edge". The Journal of cell biology 183.5 (2008), pp. 949-961. DOI: 10.1083/jcb. 200808105.

[129] A. Mukai, A. Ichiraku and K. Horikawa. "Reliable handling of highly A/T-rich genomic DNA for efficient generation of knockin strains of Dictyostelium discoideum". BMC biotechnology 16.1 (2016), p. 37. DOI: $10.1186 /$ s12896-016-0267-8.

[130] T. Gregor, K. Fujimoto, N. Masaki and S. Sawai. "The onset of collective behavior in social amoebae". Science 328.5981 (2010), pp. 1021-1025. DOI: 10.1126/science.1183415.

[131] Y. Ohta, T. Furuta, T. Nagai and K. Horikawa. "Red fluorescent cAMP indicator with increased affinity and expanded dynamic range". Scientific reports 8.1 (2018), p. 1866. DOI: $10.1038 /$ s41598-018-20251-1.

[132] A. Gholami, O. Steinbock, V. Zykov and E. Bodenschatz. "Flow-Driven Waves and PhaseLocked Self-Organization in Quasi-One-Dimensional Colonies of Dictyostelium discoideum". Physical Review Letters 114.1 (2015), p. 018103. DOI: 10.1103/PhysRevLett.114.018103.

[133] K. Kamino, Y. Kondo, A. Nakajima, M. Honda-Kitahara, K. Kaneko and S. Sawai. "Foldchange detection and scale invariance of cell-cell signaling in social amoeba". Proceedings of the National Academy of Sciences 114.21 (2017), E4149-E4157. DOI: 10.1073/pnas.1702181114. 



\section{Acknowledgments}

First and foremost I would like to thank my adviser Dr. Azam Gholami, without whom this work would not have been possible. Her guidance and support were essential during this three and a half years. I also thank Professor Eberhard Bodenschatz for giving me the opportunity to join his group and provide an environment where the symbiosis between experiments and theory that was fundamental for this work could flourish.

For the scientific discussions I thank Dr. Albert Bae and Dr. Vladimir Zykov, along with my thesis committee, Professors Klumpp and Enderlein.

Gerhard Nolte for all the technical computational assistance, and specially for saving my work when my computer was stolen.

I thank DAAD for funding most of this project, and my previous language learning stage.

I also thank other scientists who where essential in my formation and helped me in different ways along these years, they are Prof. Marcel Clerc, Prof. Claudio Falcón, Prof. Felipe Barra, Prof. Judith Lisoni, Prof. Patricio Cordero, Prof. Heinrich Jagger, Prof. Stefania Residori, Dr. Raouf Barbosa, and Ana Rubio.

Finally, here comes the unapologetically long list of people that deserves a thank you for being there and helping along the way: Daniela Pavéz, Felipe Smith, Ignacio Maldonado, Alfredo García, Renzo Fuenzalida, JT Méndez, Francisco Romero, The EuroTrip Crew (Felipe Subiabre, Nicolás Sanhueza, Sebastián Barbieri, and Ignacio Vergara), The CoC Group, Mirna Kramar, Felix Bäuerle, Felix Meigel, Natalie Andrew and Bryan Knep, Jason Khadka, Jean-Daniel Julien, George Datseris, Asnar and Carsten, Helga, Rodrigo Galilea, Antonio Ibañez, Nadine Kamprad, the DnD Groups, Dr. Christian Westendorf, Christian Küchler, Dr. Octavio Albarrán, and the PhD office people. 
